\title{
Knowledge-Driven Board-Level Functional Fault Diagnosis
}

\author{
by \\ Fangming Ye \\ Department of Electrical and Computer Engineering \\ Duke University \\ Date: \\ Approved:
}

\begin{tabular}{c}
\hline Krishnendu Chakrabarty, Supervisor \\
\hline Jeffrey H. Derby \\
\hline Kishor S. Trivedi \\
\hline Martin A. Brooke \\
\hline Xinli Gu
\end{tabular}

Dissertation submitted in partial fulfillment of the requirements for the degree of Doctor of Philosophy in the Department of Electrical and Computer Engineering in the Graduate School of Duke University

2014 


\title{
$\underline{\text { ABSTRACT }}$
}

\section{Knowledge-Driven Board-Level Functional Fault Diagnosis}

\author{
by \\ Fangming Ye \\ Department of Electrical and Computer Engineering \\ Duke University \\ Date: \\ Approved:
}

Krishnendu Chakrabarty, Supervisor

Jeffrey H. Derby

\begin{tabular}{c}
\hline Kishor S. Trivedi \\
\hline Martin A. Brooke \\
Xinli Gu
\end{tabular}

An abstract of a dissertation submitted in partial fulfillment of the requirements for the degree of Doctor of Philosophy in the Department of Electrical and Computer Engineering

in the Graduate School of Duke University

2014 
Copyright (C) 2014 by Fangming Ye

All rights reserved except the rights granted by the Creative Commons Attribution-Noncommercial Licence 


\section{Abstract}

The semiconductor industry continues to relentlessly advance silicon technology scaling into the deep-submicron (DSM) era. High integration levels and structured design methods enable complex systems that can be manufactured in high volume. However, due to increasing integration densities and high operating speeds, subtle manifestation of defects leads to functional failures at the board level. Functional fault diagnosis is, therefore, necessary for board-level product qualification. However, ambiguous diagnosis results can lead to long debug times and wrong repair actions, which significantly increase repair cost and adversely impact yield.

A state-of-the-art diagnosis system involves several key components: (1) design of functional test programs, (2) collection of functional-failure syndromes, (3) building of the diagnosis engine, (4) isolation of root causes, and (5) evaluation of the diagnosis engine. Advances in each of these components can pave the way for a more effective diagnosis system, thus improving diagnosis accuracy and reducing diagnosis time. Machine-learning techniques offer an unprecedented opportunity to develop an automated and adaptive diagnosis system to increase diagnosis accuracy and speed. This dissertation targets all the above components of an advanced diagnosis system by leveraging various machine-learning techniques.

This thesis first describes a diagnosis system based on support-vector machines (SVMs), multi-kernel SVMs (MK-SVMs) and incremental learning. The MK-SVM method leverages a linear combination of single kernels to achieve accurate root-cause 
isolation. The MK-SVMs thus generated also can be updated based on incremental learning. Furthermore, a data-fusion technique, namely majority-weighted voting, is used to leverage multiple learning techniques for diagnosis.

The diagnosis time is considerable for complex boards due to the large number of syndromes that must be used to ensure diagnostic accuracy. Syndrome collection and analysis are major bottlenecks in state-of-the-art diagnosis procedures. Therefore, this thesis describes an adaptive diagnosis method based on decision trees (DT). The number of syndromes required for diagnosis can be significantly reduced compared to the number of syndromes used for system training. Furthermore, an incremental version of DTs is used to facilitate online learning, so as to bridge the knowledge obtained at test-design stage with the knowledge gained during volume production.

This dissertation also includes an evaluation and enhancement framework based on information theory for guiding diagnosis systems using syndrome and root-cause analysis. Syndrome analysis based on subset selection provides a representative set of syndromes. Root-cause analysis measures the discriminative ability of differentiating a given root cause from others. The metrics obtained from the proposed framework can provide guidelines for test redesign to enhance diagnosis. In addition, traditional diagnosis systems fail to provide appropriate repair suggestions when the diagnostic logs are fragmented and some syndromes are not available. The feature of handling missing syndromes based on imputation methods has therefore been added to the diagnosis system.

Finally, to tackle the bottleneck of data acquisition during the initial product ramp-up phase, a knowledge-discovery method and a knowledge-transfer method are proposed for enriching the training data set, thus facilitating board-level functional fault diagnosis. In summary, this dissertation targets the realization of an automated diagnosis system with the features of high accuracy, low diagnosis time, self-evaluation, self-learning, and ability of selective learning from other diagnosis 
systems. Machine learning and information-theoretic techniques have been adopted to enable the above-listed features. The proposed diagnosis system is expected to contribute to quality assurance, accelerated product release, and manufacturing-cost reduction in the semiconductor industry. 


\section{Contents}

Abstract $\quad$ iv

List of Tables $\quad$ xi

List of Figures xiii

$\begin{array}{ll}\text { Acknowledgements } & \text { xvii }\end{array}$

1 Introduction $\quad 1$

1.1 Overview of Manufacturing Test . . . . . . . . . . . . . 2

1.1.1 System and Tests . . . . . . . . . . . . . . . 2

1.1.2 Testing in the Manufacturing Line. . . . . . . . . . . . 4

1.2 Overview of Board-Level Diagnosis . . . . . . . . . . . . . . . 9

1.2.1 Overview of State-of-The-Art . . . . . . . . . . . . . . 10

1.2.2 Automated Diagnosis System . . . . . . . . . . . . 15

1.3 Research Challenges and Motivation . . . . . . . . . . . . 19

1.4 Outline of Dissertation . . . . . . . . . . . . . . . 22

2 Diagnosis Using Support-Vector Machines 25

2.1 Problem Statement and Prior Work . . . . . . . . . . . . 26

2.2 Diagnosis Using Support-Vector Machines . . . . . . . . . . . . 28

2.2.1 Support-Vector Machines . . . . . . . . . . . . . 29

2.2.2 SVM Diagnosis Flow . . . . . . . . . . . . . . . . 32

2.3 Multi-Kernel Support-Vector Machines and Incremental Learning . . 34 
2.3.1 Multi-Kernel Support-Vector Machines . . . . . . . . . . . . . 34

2.3.2 Incremental Learning . . . . . . . . . . . . . . . . . . . 37

2.4 Experiments and Results . . . . . . . . . . . . . . . . . . . 40

2.5 Conclusions . . . . . . . . . . . . . . . . . . . . . 51

3 Diagnosis Using Multiple Classifiers and Majority-Weighted Voting 52

3.1 Problem Statement and Chapter Contributions . . . . . . . . . . 53

3.2 Artificial Neural Networks . . . . . . . . . . . . . . . 56

3.3 Comparison between ANNs and SVMs . . . . . . . . . . . 60

3.4 Diagnosis Using Weighted-Majority Voting . . . . . . . . . . . . . 61

3.4.1 Weighted-Majority Voting ............. 61

3.4.2 Demonstration of WMV-Based Diagnosis System . . . . . . . 63

3.5 Experiments and Results . . . . . . . . . . . . . . 64

3.6 Conclusions .......................... 74

4 Adaptive Diagnosis Using Decision Trees $\quad 76$

4.1 Problem Statement and Chapter Contributions . . . . . . . . . . 77

4.2 Decision Trees . . . . . . . . . . . . . . . . . . . . . . . . 79

4.2.1 Training of Decision Trees . . . . . . . . . . . . . . . 80

4.2.2 Example of DT-based Training and Diagnosis . . . . . . . . . 82

4.3 Diagnosis Using Incremental Decision Trees . . . . . . . . . . . . . 84

4.3.1 Incremental Tree Node . . . . . . . . . . . . . . . . . 85

4.3.2 Addition of a Case . . . . . . . . . . . . . . . . . . 87

4.3.3 Ensuring the Best Splitting . . . . . . . . . . . . . 89

4.3.4 Tree Transposition . . . . . . . . . . . . . . . 91

4.4 Diagnosis Flow Based on Incremental Decision Trees . . . . . . . . . 91

4.5 Experiments and Results . . . . . . . . . . . . . . . 94 
4.6 Conclusions . . . . . . . . . . . . . . . . . . . . . . . . . . . 99

5 Handling Missing Syndromes 101

5.1 Introduction . . . . . . . . . . . . . . . . . . . . . 101

5.2 Problem Statement and Chapter Contributions _ . . . . . . . . 102

5.3 Methods to Handle Missing Syndromes . . . . . . . . . . . . . 105

5.3 .1 Numerical Imputation . . . . . . . . . . . . . . . 107

5.3 .2 Label Imputation . . . . . . . . . . . . . . . . . . 110

5.4 Experiments and Results . . . . . . . . . . . . . . . . 111

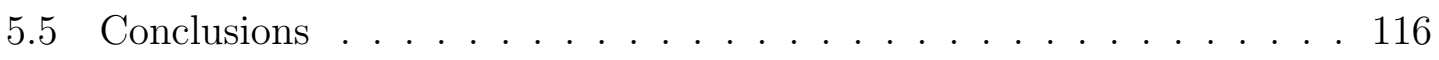

6 Information-Theoretic Syndrome and Root-Cause Evaluation 117

6.1 Problem Statement and Chapter Contributions _. . . . . . . . . 119

6.2 Evaluation Methods for Diagnosis Systems . . . . . . . . . . . . . 121

6.2.1 Subset Selection for Syndromes Analysis . . . . . . . . . . 121

6.2 .2 Class-Relevance Statistics ． . . . . . . . . . . . . . . 124

6.3 Evaluation and Enhancement Framework . . . . . . . . . . . 126

6.4 Experiments and Results . . . . . . . . . . . . . . . . . . . 129

$6.4 .1 \quad$ Experimental setup . . . . . . . . . . . . . . . . 129

6.4 .2 Results . . . . . . . . . . . . . . . . . 130

6.5 Conclusions . . . . . . . . . . . . . . . . . . 136

$\begin{array}{lll}7 & \text { Knowledge Discovery and Knowledge Transfer } & 137\end{array}$

7.1 Problem Statement and Chapter Contributions _ . . . . . . . 138

7.2 Overview of Knowledge Discovery and Transfer Framework . . . . . . 140

7.3 Knowledge-Discovery Method . . . . . . . . . . . . . . . . . . 142

7.4 Knowledge-Transfer Method . . . . . . . . . . . . . . . 147

7.5 Experimental Results . . . . . . . . . . . . . . 155 
7.5.1 Experimental Setup . . . . . . . . . . . . . . 155

7.5.2 Results and Discussion . . . . . . . . . . . . . . 156

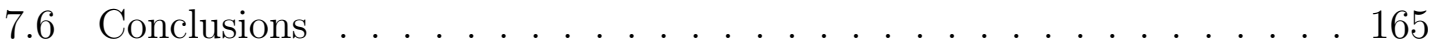

8 Conclusions and Future Work 166

8.1 Thesis Contributions . . . . . . . . . . . . . 167

8.2 Future Work . . . . . . . . . . . . . . . . . . . 169

$\begin{array}{lr}\text { Bibliography } & 172\end{array}$

$\begin{array}{ll}\text { Biography } & 180\end{array}$ 


\section{List of Tables}

1.1 Rule of ten in test economics $[1] \ldots \ldots \ldots \ldots$

2.1 Information about the industrial boards used for classification and the $\log$ data available. . . . . . . . . . . . . . . . . . 4 41

2.2 Diagnosis results using ANN, SVMs with different kernel functions,

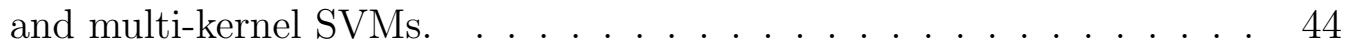

3.1 Information about the industrial boards used for classification and the $\log$ data available. . . . . . . . . . . . . . 65

3.2 Diagnosis results for the ANN-based system. . . . . . . . . . 66

3.3 Comparison of SRs obtained by ANNs and BI. . . . . . . . . . 67

3.4 Comparison of SRs obtained by SVMs with different kernels. . . . . . 69

3.5 Comparison of SRs obtained by ANNs, SVMs, and WMV. . . . . . 72

4.1 Information about the industrial boards used for classification and the $\log$ data available. . . . . . . . . . . . . . . . . 94

4.2 Comparison of SRs and number of syndromes obtained by ANNs, SVMs and DTs with different splitting criteria. . . . . . . . 95

4.3 Comparison of DTs and SVMs with reduced set of syndromes. . . . 96

4.4 Comparison of DTs and ANNs with reduced set of syndromes. . . . . 97

5.1 Information about the synthetic boards used for classification. . . . . 111

6.1 Confusion matrix. . . . . . . . . . . . . . . . . . . . 124

6.2 Diagnosis results before and after evaluation for a diagnosis system based on SVMs. . . . . . . . . . . . . . . . . . 130 
6.3 Diagnosis results before and after evaluation for a diagnosis system based on ANNs. . . . . . . . . . . . . . . . . . . . 131

7.1 Information about the industrial boards with mature diagnosis engines. 155

7.2 Information about the industrial boards at the ramp-up stage. . . . . 155 


\section{List of Figures}

1.1 A typical manufacturing test line for electronic systems. . . . . . . . . 6

1.2 Illustration of the boundary-scan test architecture. . . . . . . . . . . 8

1.3 Illustration of rule-based diagnosis methods. . . . . . . . . . . . . . . 10

1.4 Illustration of model-based diagnosis methods. . . . . . . . . . . . . . 12

1.5 Illustration of reasoning-based diagnosis methods. . . . . . . . . . . . 13

1.6 Illustration of new product ramp-up with and without reasoning-based tools $[2] \ldots \ldots \ldots \ldots \ldots$

1.7 Flowchart for automated diagnosis. . . . . . . . . . . . . . 16

1.8 A segment of the $\log$ file for a failed functional test. . . . . . . . . . . 18

2.1 Illustration of a 2-class support-vector machine model. . . . . . . . . 30

2.2 The diagnosis flow using SVMs [3]. . . . . . . . . . . . . . . 33

2.3 Illustration of kernelized support-vector machine model. . . . . . . . . 36

2.4 Illustration of training a multikernel SVM. . . . . . . . . . . . . 37

2.5 Illustration of the proposed iMK-SVM approach for fault diagnosis. . 39

2.6 Comparison of training time in each epoch between incremental and non-incremental linear-kernel SVMs for Board 1. . . . . . . . . . . 45

2.7 Comparison of training time in each epoch between incremental and non-incremental linear-kernel SVMs for Board 2. . . . . . . . . . . . 45

2.8 Comparison of $\mathrm{SR}_{1}$ between incremental and non-incremental linearkernel SVMs for Board 1. . . . . . . . . . . . . . . . . . . 46

2.9 Comparison of $\mathrm{SR}_{1}$ between incremental and non-incremental linearkernel SVMs for Board 2. . . . . . . . . . . . . . . . . 46 
2.10 Success ratio of using incremental multi-kernel SVMs for Board 1. . . 47

2.11 Success ratio of using incremental multi-kernel SVMs for Board 2. . . 48

2.12 Comparison of training time in each epoch between using incremental and non-incremental multi-kernel SVMs for Board 1. . . . . . . . . . 49

2.13 Comparison of training time in each epoch between using incremental and non-incremental multi-kernel SVMs for Board 2. . . . . . . . . . 49

2.14 Illustration of the change in kernel weights in incremental multi-kernel SVMs for Board 1. . . . . . . . . . . . . . . . . . 50

2.15 Illustration of the change in kernel weights in incremental multi-kernel SVMs for Board 2. . . . . . . . . . . . . . . . . . . 50

3.1 A simple feed-forward neural network and the computation in a neuron [4]. . . . . . . . . . . . . . . . . . 56

3.2 An illustration of the proposed ANN architecture [4]. . . . . . . . . . 57

3.3 The diagnosis flow using neural networks [4] . . . . . . . . . . . . . . 59

3.4 Illustration of weighted-majority voting. . . . . . . . . . . . . 62

3.5 SR improvement when WMV is used instead of ANNs. . . . . . . . . 73

3.6 SR improvement when WMV is used instead of SVMs. . . . . . . . . 73

4.1 Illustration of the benefit of bridging the knowledge gap between debug technicians and an automated learning system. . . . . . . . . . . 78

4.2 Illustration of a decision tree. . . . . . . . . . . . . . . . . . 80

4.3 DT trained for demo board. . . . . . . . . . . . . . . . 84

4.4 Outline of the training algorithm for iDTs. . . . . . . . . . . . . 84

4.5 Illustration of nodes with additional scores for incremental learning. . 85

4.6 Procedure for adding a new case to a DT. . . . . . . . . . . 86

4.7 Illustration of the addition of a case (Scenario I) . . . . . . . . . . . . 87

4.8 Illustration of the addition of a case (Scenario II) . . . . . . . . . . . . 88

4.9 Pseudo-code for the algorithm ensure_best_splitting ()$\ldots \ldots$. . . . . . . 90

4.10 Illustration of the tree-transposition procedure. . . . . . . . . . . . . 90 
4.11 The diagnosis flow using DTs. . . . . . . . . . . . . . . . . . 92

4.12 Diagnosis of new cases using DTs. . . . . . . . . . . . . . . . . . . 94

4.13 Comparison of fault diagnosis using batch DTs, incremental DTs, incremental DTs* for Board 1. . . . . . . . . . . . . . . . . 98

4.14 Comparison of fault diagnosis using batch DTs, incremental DTs, incremental DTs* for Board 2. . . . . . . . . . . . . . . . . . . . . . 98

5.1 Illustration of parsing a log with and without missing syndromes. . . 103

5.2 The SVM-based diagnosis flow with the capability of handling missing syndromes. . . . . . . . . . . . . . . . . . 105

5.3 Comparison of success ratios for diagnosis using label imputation with different weights for Board 1. . . . . . . . . . . . . 113

5.4 Comparison of success ratios for diagnosis using label imputation with different weights for Board 2. . . . . . . . . . . . . . 113

5.5 Comparison of success ratios for diagnosis using different imputation methods for Board 1. . . . . . . . . . . . . . . . . . . . 114

5.6 Comparison of success ratios for diagnosis using different imputation methods for Board 2. . . . . . . . . . . . . . . . . . . . 114

5.7 Comparison of training time for diagnosis system using different imputation methods for Board 1. . . . . . . . . . . . . . . . 115

5.8 Comparison of training time for diagnosis system using different imputation methods for Board 2. . . . . . . . . . . . . . . . . . 115

6.1 Overview of the diagnosis system with self-evaluation. . . . . . . . 118

6.2 Evaluation framework for a diagnosis system and the targeted problems.119

6.3 Selection of a representative syndrome subset. . . . . . . . . . . . . . 122

6.4 Precision and recall distribution for an SVM-based diagnosis system for three boards. . . . . . . . . . . . . . . . . . . . . . . . . . 132

6.5 Precision and recall distribution for an ANN-based diagnosis system for three boards. . . . . . . . . . . . . . . . . . . . . 133

6.6 Comparison of ambiguous root-cause pairs for an SVM-based diagnosis system for three boards. . . . . . . . . . . . . . . . . . . 134 
6.7 Comparison of ambiguous root-cause pairs for an ANN-based diagnosis system for three boards. . . . . . . . . . . . . . . . . . . 135

7.1 The problem of low accuracy for a reasoning-based diagnosis system at the beginning of a new product (Product 3) ramp-up stage. . . . . 138

7.2 Illustration of knowledge-discovery and transfer framework for boardlevel diagnosis. . . . . . . . . . . . . . . . . . . . . . . . 140

7.3 A segment of the $\log$ file for a failed functional test. . . . . . . . . . 143

7.4 Flowchart of knowledge-discovery method. . . . . . . . . . . . . . . . 143

7.5 A segment of the $\log$ file for a failed functional test. . . . . . . . . . . 144

7.6 Two-stage knowledge transfer from an old diagnosis system to a new diagnosis system. . . . . . . . . . . . . . . . . 148

7.7 Illustration of a mature board from which knowledge can be transferred.149

7.8 Illustration of a new board that needs knowledge transfer. . . . . . . 149

7.9 Illustration of tests on a mature board with knowledge to transfer from.150

7.10 Illustration of tests on a new board that needs knowledge transfer. . . 151

7.11 Proposed knowledge-transfer algorithm. . . . . . . . . . . . . . . 152

7.12 Comparison of diagnosis accuracy using SVMs between manual method, knowledge discovery, knowledge transfer, and the hybrid method. . . 157

7.13 Comparison of diagnosis accuracy using ANNs between manual method, knowledge discovery, knowledge transfer, and the hybrid method. . . 158

7.14 Comparison of diagnosis accuracy using DTs between manual method, knowledge discovery, knowledge transfer, and the hybrid method. . . 159

7.15 Precision and recall distribution for an SVM-based diagnosis system for Board 1 (Part I). . . . . . . . . . . . . . . . . . 163

7.16 Precision and recall distribution for an SVM-based diagnosis system for Board 1 (Part II) . . . . . . . . . . . . . . . . . . . . . . 164 


\section{Acknowledgements}

I would like to express my deep gratitude to Professor Krishnendu Chakrabarty, for his patient guidance, enthusiastic encouragement, and useful critiques of this research work. Krish is a role model for me as a real researcher, a thinker, and a professional. The knowledge and the way of thinking and solving problems I learned from him will benefit all my academic life. This thesis and most publications would not be accomplished without Krish's extensive editing and critiques. I would also like to thank my mentor, Dr. Zhaobo Zhang, for her advice and kindness during my study. I am very grateful to Professors Jeffrey H. Derby, Kishor S. Trivedi, Martin A. Brooke, and Dr. Xinli Gu for being my committee members and giving me constructive suggestions.

My special thanks go to Dr. Xinli $\mathrm{Gu}$, who is always there to support me and he provided immense contributions to this thesis research. My three times internship in Huawei technologies has broadened my vision on practical issues in manufacturing tests and use of knowledge-driven techniques in DFX. I would also like to thank my colleagues, Gang Chen, Xiao Liu, Brian Wang, Yaohui Xie, and Tao Xu in Huawei Techniques(USA) for their various helps.

My appreciation also go to my peers and long-time officemates, Mukesh Agrawal, Sergej Deutsch, Dr. Qing Duan, Kai Hu, Zipeng Li, Dr. Yan Luo, Dr. Brandon Noia, and Ran Wang for their helps and beneficial discussions at Duke. I would also like to extend my appreciation to my research collaborators, Dr. Li Jiang from 
Shanghai Jiaotong University, China and Farshad Firouzi from KIT, Germany for their academic and personal helps.

I would like to thank ECE staff, Amy Kostrewa, Samantha Morton, Ellen Currin, Paul McClain, Mary Pope, and Thomas Napolas for their helps when I needed them.

Financial supports from Huawei Technologies, Semiconductor Research Corporation are acknowledged.

Finally, I would like to express my gratitude and deepest love to my parents and grandparents, who are always there sharing my joys and pains. My last thank is for Liyuan, my wife and soul, for your endless support, love, and trust. 


\section{Introduction}

Relentless technology scaling has increased the performance and complexity of electronic products by orders of magnitude in the past few decades. A complex system today consists of several chassis, and each of them contains a number of printedcircuit boards (PCBs). A typical board consists of many application-specific integrated circuits (ASICs) and memory devices. Each ASIC in turn consists of hundreds of inputs/outputs (I/Os), millions of logic gates, and several tens of millions of bits of embedded memory. Moreover, the operating frequencies of high-speed ASICs are above $1 \mathrm{GHz}$, and the data rates of high-speed I/Os are up to 6 Gbps [5,6]. With increasing complexity and higher speeds, defective-parts-per-million (DPPM) rates continue to increase and subtle functional failures are becoming increasingly difficult to detect and diagnose for root-cause identification $[7,8]$.

In this chapter, we provide motivation for the thesis research and introduce basic concepts and terminology. Section 1.1 presents an overview of manufacturing test and diagnosis. Section 1.2 discusses the state-of-the-art in board-level diagnosis. Section 1.3 presents the challenges of board-level diagnosis and motivation for this research. Finally, an outline of this thesis is provided in Section 1.4. 


\subsection{Overview of Manufacturing Test}

\subsubsection{System and Tests}

This section presents some basic definitions that are used in system test and diagnosis $[9,10]$.

- Definition 1. A system $\mathbf{S}$ is defined as a heterogeneous collection of cooperating entities, designed to realize a specified group of functionalities.

Definition 1 is quite general and it can be adopted to cover a set of different types of systems in different fields of engineering, from digital systems to computer networks, nuclear facilities, and chemical plants $[9,10]$.

- Definition 2. A fault $\mathbf{f}$ is defined as a source of misbehavior of a system. The presence of a fault puts the system in a state where the execution of one or more of its functionalities is different from the expected execution.

In the reliability literature [11], there is a distinction between defects, which correspond to locations of a system instance containing a difference between specification and implementation, faults, defined as the actual causes of a system misbehavior, and failures, representing the external observable effects of a fault, producing a deviation of functionality from specifications.

- Definition 3. Diagnosis refers to the process of determining the causes of system failure.

In order to describe the diagnosis process, some additional definitions are needed, covering both the targets of this process (components) and the information required to be collected to execute diagnosis (tests). These concepts will be introduced later.

- Definition 4. A component $\mathbf{c}$ represents a part of system. 
Let $\mathbf{C}_{\mathrm{S}}=\left\{\mathbf{c}_{1}, \mathbf{c}_{2}, \mathbf{c}_{3} \ldots \mathbf{c}_{n}\right\}$ be the set of all components that constitute system $\mathbf{S}$. A component containing (at least) one fault is referred to as a root-cause component; every other component is fault-free.

Definition 4 requires that each component must be identified unambiguously within the system. An extension of Definition 2 is that a fault can affect only one component, and two components of a system cannot overlap.

According to the above definitions, a component can correspond to a physical device in the system (e.g., a memory chip in a digital system). However, this condition is not strictly required. A component can also refer to a group of physically connected elements within the system, or even a set of non-interconnected entities contained in a system (virtual component).

- Definition 5. A subcomponent $\mathbf{s c}$ represents a part within a component $\mathbf{c}$ of system $\mathbf{S}$.

Note that the relationship between components and subcomponents is similar to the relationship between system and components, since it introduces a partition of the fault set of each component.

- Definition 6. A (diagnostic) test $\mathbf{t}$ represents a measurement of a property of system $\mathbf{S}$, targeting the identification of the syndromes corresponding to the presence of a fault.

Let $\mathbf{T}_{\mathrm{S}}=\left\{\mathbf{t}_{1}, \mathbf{t}_{2}, \mathbf{t}_{3} \ldots \mathbf{t}_{n}\right\}$ denote the set of all tests used for system $\mathrm{S}$, also referred to as Test Suite. In general, testing a system can be described as the operation of performing a set of tests [9]. In a diagnostic environment, tests are designed specifically to identify a potential syndrome of a failure, in order to make the defect observable within the system.

- Definition 7. A syndrome $\mathbf{s}$ characterizes the measurement of a test $\mathbf{t}$. 
Syndromes can provide detailed information about system parameters (e.g., the value of a sensor measurement [12]) or the output values of a circuit [13]. In some cases, the information can be compacted in some form in order to keep only its most significant part, e.g., the correspondence between the observed value of the parameter and its expected one. When maximally compacted, a test outcome is a binary variable that takes the value pass or fail.

- Definition 8. A root cause is the faulty component that leads to the failure of system $\mathbf{S}$.

The isolation of a root cause is achieved on the basis of the syndromes observed for some or for all tests $\mathbf{T}_{\mathrm{S}}$. These syndromes contain the information that can be used to reveal the cause of misbehavior in system $\mathbf{S}$.

A common assumption adopted in diagnosis is the so-called single fault hypothesis. This assumption is necessary in order to reduce the complexity of considering an exponential number of fault combinations to describe all possible system failures. According to this assumption, any instance of a system can contain at most one root-cause component.

\subsubsection{Testing in the Manufacturing Line}

Manufacturing test ensures that electronic products have no defects before they are shipped to customers. Each fabricated product is subject to manufacturing test. The process of manufacturing test is described as the explanation or interpretation of a set of failure symptom [14], where a sequence of diagnostic tests is selected efficiently to locate the root causes of failures. A diagnosis strategy has to tackle the following fundamental issues $[15]$ :

- Detection, i.e., the capability of a combination of tests to identify the presence of a fault in a system. 
- Isolation, i.e., the capability of a diagnostic strategy to achieve fine-grained localization in order to allow the repair of a single component.

Compared to detection, isolation is a more difficult task in manufacturing test and it is the main focus of this work. In this thesis, Diagnosis refers to root-cause isolation.

Diagnosis strategies vary across the different stages of system assembly. In order to better understand the problems and challenges at the board level, let us first examine chip-level testing. At the chip level, scan test is commonly used. In order to shift test patterns and response in and out of the chip, respectively, all the flip-flops are stitched to one or multiple scan chains. Test patterns are generated by automatic test-pattern generation (ATPG) tools and scanned into the circuit under test (CUT). The response of the circuit is compared with the expected response. The circuit is deemed to be fault-free if the test response matches the expected response. This testing process can be performed on automatic test equipment (ATE).

A modern ATE is a complex equipment operating at $\mathrm{GHz}$ frequency and with high throughput. The test cost on a high-end ATE can be up to thousands of dollars per pin [10]. Therefore, test-data compression techniques have been widely explored in the literature, in order to reduce the volume of test patterns and testing time. To reduce dependence of an expensive ATE, built-in-self-test (BIST) techniques have also been used [10]. In BIST, pseudo-random patterns are generated on-chip using a pattern generation circuit, e.g., a linear-feedback shift register (LFSR). The on-chip pattern generation approach eliminates the need for expensive external testers, and makes high-speed test possible. However, the reliance on pseudorandom patterns to achieve adequate fault coverage leads to a large volume of test data, compared to the use of deterministic patterns generated by ATPG tools. 


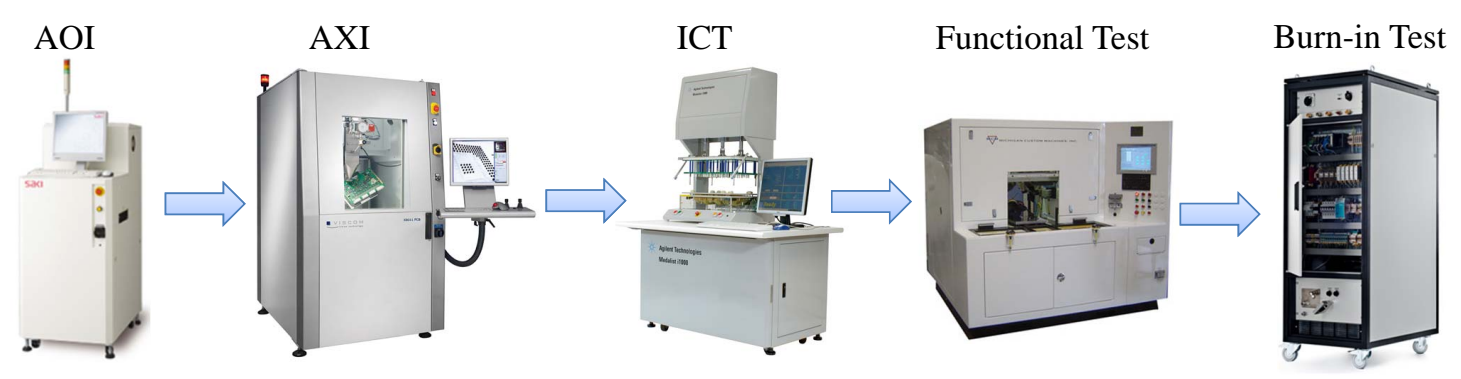

FiguRE 1.1: A typical manufacturing test line for electronic systems.

Chip-level test has been well studied in the past. The testing goal is straightforward and quantifiable, i.e., to detect a high percentage of defects that are introduced during manufacturing. The test environment (ATE) is well-understood and a given number of pins are available for probing. Sophisticated tools and methodologies are available for inserting effective design-for-testability (DFT) structures and for automatically generating test patterns to get the desired fault coverage. The focus of this research is on the testing and diagnostic problems at higher integration levels. Much less attention has been devoted in the literature to these problems.

Circuit boards consist of previously-tested components. An important objective of board testing is to verify the printed wiring and the contacts between wires and components. Moreover, the at-speed interactions between the components must also be tested. A typical manufacturing test line of electronic systems is shown in Figure 1.1. Testing starts on the left-hand side of the figure. On the right-hand side, completely tested products are placed in inventory or shipped to customers. The testing process is separated into multiple stages to provide better failure isolation and feedback to the manufacturing process.

Process test, such as automated optical inspection (AOI) and automated X-ray inspection (AXI), is first applied to immediately catch process flaws, e.g., solder shorts, unreliable solder joints. In-circuit test (ICT) is used to verify the performance of individual components using a bed-of-nails fixture. The bed-of-nails fixture is used 
to gain access to the board. ICT is useful to guarantee that a component receives the correct value, since many board failures are caused by open/short circuits or wrong components. Functional test, which targets the functional correctness of components and the whole system, is typically run after process tests. Sometimes, system test is performed as the final test, which is also a type of functional test. Each test technique has its advantages. For example, the solder reliability can be easily checked by AXI. The reversed or inoperative components can be detected by ICT. No single test can, however, cover all possible defects.

With the development of high density assembly on PCBs, the number of access points for in-circuit test keeps decreasing. An interconnection test method, namely boundary-scan test, has been widely adopted by manufacturers. The boundary-scan architecture is defined in the IEEE 1149.1 and 1149.6 standards $[16,17]$ to ensure connectivity between components. Simple boards and complex multi-board systems can effectively be tested using the IEEE 1149.6 standard-compliant equipment from the product design phase to mainstream manufacturing. At present, there is an increased focus in the electronics industry on using the concept of remote test and diagnosis in order to provide a mechanism that allows continued support of a product [18]. The boundary-scan test architecture is illustrated in Figure 1.2. A boundaryscan cell, which includes a multiplexer and latches, is added to each pin on the chip. Boundary-scan cells can capture data from pins or core logic signals, and force data to pins. The test data is serially shifted into the boundary-scan cells. Then, the captured data is serially shifted out and externally compared with the expected responses. Boundary scan allows for full controllability and observability of board wires and therefore enables a simple interconnect test with a high fault coverage.

Despite the benefits offered by the above techniques, individual component testing and interconnection testing are no longer sufficient to guarantee the quality of complex circuit boards $[19,20]$. It is often the case that all the chips on a board 


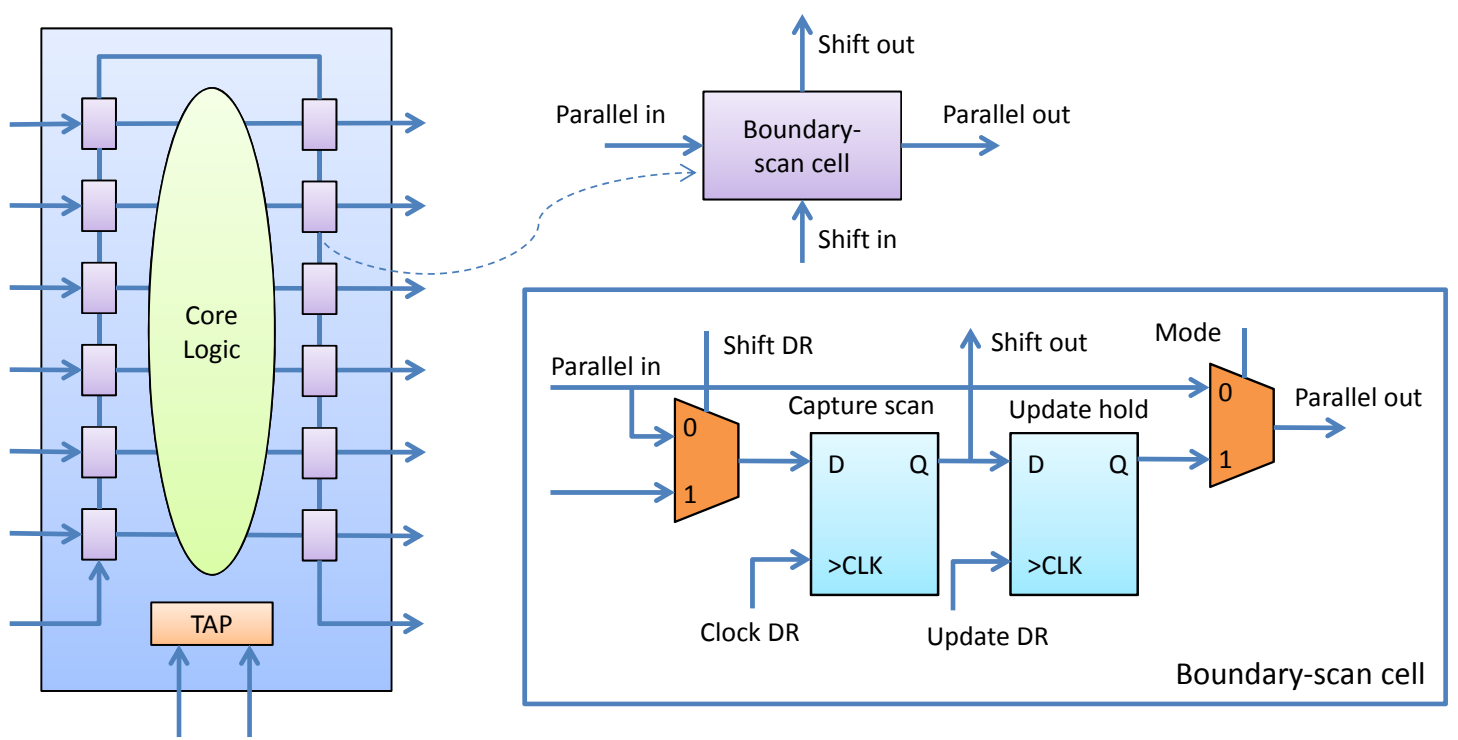

FiguRE 1.2: Illustration of the boundary-scan test architecture.

pass ATE tests, but the board still fails functional test, a scenario referred to as "No Trouble found" (NTF). The reason for NTF is that the board-level test environment is different from that at the chip level. At the chip manufacturing sites, chips are tested in a standalone mode, but additional issues, e.g., signal integrity, power-supply noise, and crosstalk involving multiple active chips, must be considered in board-level test [21]. The main objective of functional tests is to detect NTFs and verify the functional correctness of a board. Functional test plays an important role in capturing defects that cannot be easily detected by structural test.

Functional tests are indispensable today in system test and diagnosis [5]. Typically, functional test targets only a subset of all the designed functions, usually the critical functions identified by designers. Functional test sequences are often derived from design verification programs, and they are close to the practical scenarios that occur in the field. With the need for high throughput and efficient manufacturing, sophisticated functional-test platforms based on open industry standards are required by electronics contract manufacturers and equipment manufacturers. 


\subsection{Overview of Board-Level Diagnosis}

Fault diagnosis isolates the root cause of a malfunction system by collecting and analyzing information on system status using measurements, tests, and other information sources. It is important at all stages of the product life cycle, but particularly crucial during manufacture and field maintenance.

The degree of accuracy with which faults can be located is referred to as diagnostic resolution [13]. Diagnostic success ratio refers to the ratio of the number of correctly diagnosed cases to the total number of cases under diagnosis. The diagnosis process can be hierarchically carried out as a top-down process (with a system operating in the field) or a bottom-up process (during the fabrication of a system). In the top-down process (system $\rightarrow$ boards $\rightarrow$ chips), the first-level diagnosis usually deals with large units such as boards. The faulty board is then tested to locate the faulty component on the board. Accurate location of faults inside a faulty chip is important information for chip manufacturers to improve the fabrication process. In the bottom-up approach (chips $\rightarrow$ boards $\rightarrow$ system), a higher level is assembled only from components already tested at a lower level. This is done to minimize the cost of diagnosis and repair, which escalates with the level at which faults are detected.

At the chip level, existing electronic design automation tools are able to perform accurate and high-resolution diagnosis. The failure mechanism, logic location and even physical location of a fault can be determined, according to scan-test patterns [22]. The board-level diagnosis, in contrast, is much more challenging. There is no

effective flow to locate the root cause of a failure during function tests. Once a failure is detected in the manufacturing line, the failed product is sent to the diagnosis department for repair. Typically, technicians run additional functional tests and measurements based on their personal experience. This process is time-consuming, and there is no guarantee of the success of repair. In addition, a board has to be 
scrapped after a few unsuccessful repair attempts. Current diagnostic software is not able to accurately and rapidly locate the root cause. Above all, the development of the diagnostic software is increasingly challenging, as electronic systems become more complex. Developing a diagnosis software heavily relies on the experience of debug technicians, which adds to total manufacturing costs.

\subsubsection{Overview of State-of-The-Art}

A number of board-level fault diagnosis techniques have been presented in the literature $[9,12,15,23-29]$.

\section{Rule-Based Methods}

Rule-based diagnosis methods take the form "IF syndrome(s), THEN fault(s)" to locate a fault [23], as shown in Figure 1.3. Hundreds or thousands of rules may be required to represent all the relevant knowledge for the system under diagnosis. Rule-based diagnosis involves the extraction of syndromes from the failure, and the firing of rules that match the syndromes. This process is repeated iteratively until the root cause of the failure is found. Rule-based expert systems have been developed for board repair and maintenance. The primary advantage of this flow is its simplicity and its ease of implementation for small systems, where rule-based methods can provide a powerful tool for quickly filtering out least likely hypotheses. These methods do not require a systematic understanding of the underlying system

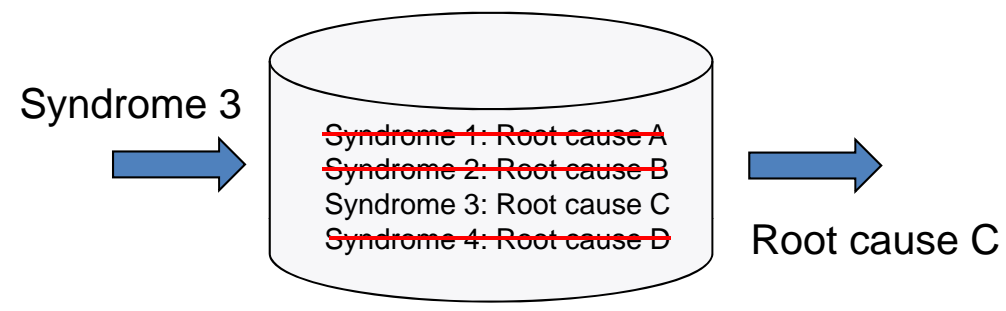

FIGURE 1.3: Illustration of rule-based diagnosis methods. 
architecture or operational principles.

However, rule-based diagnosis systems suffer from a number of disadvantages that limit their use for complex systems. They do not incorporate adaptive learning from experience and they are unable to deal with unseen scenarios. This is correlated to the inability to update system knowledge. Furthermore, rule-based methods are inefficient in dealing with inaccurate information. In hierarchical systems, the lack of a reference with respect to the system structure makes it very difficult to reuse diagnosis system developed for similar systems.

Nevertheless, these approaches are important for historical reasons, since they represent the first attempts to solve diagnostic problems; furthermore, rules are the most immediate instrument to describe failure symptoms in the form of "IF syndrome(s), THEN fault(s)" relationship to produce a systematic description of engineering expertise.

For instance, in [24], the knowledge of the proposed rule-based system is a set of If-Then rules, connecting different observations of tests to diagnostic conclusions (fault candidates). The inference is performed by a sequential rule interpreter, which activates rules according to observations, until a unique diagnostic conclusion is reached.

In [30], authors reformulated fault diagnosis on the basis of a rule-based inference, which stores the test information as a matrix. Diagnosis conclusion implies a set of failures, and a failing test requires at least one diagnosis conclusion to be true.

\section{Model-Based Methods}

Model-based methods rely on an approximate representation of the system under diagnosis, as shown in Figure 1.4. The system model is typically constructed in a hierarchical manner; higher-level models are based on lower-level models. The advantages of these methods are their robustness, straightforwardness, and ease of 


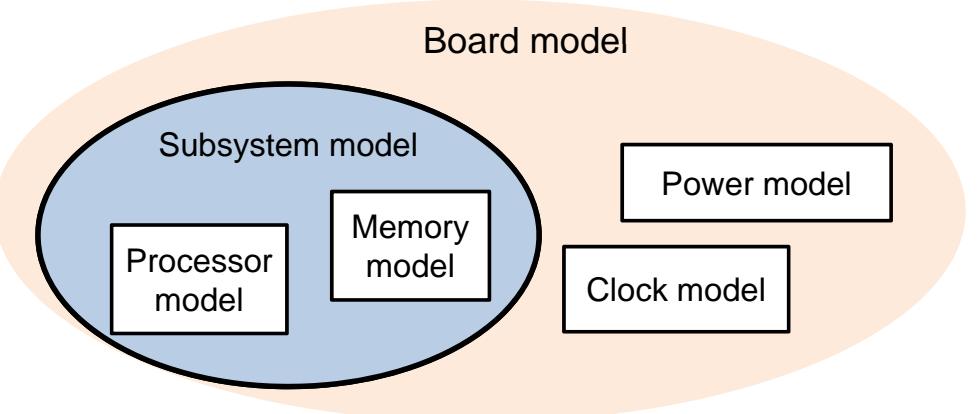

FigURE 1.4: Illustration of model-based diagnosis methods.

use. They have the potential to solve new problems and their knowledge may be organized in an expandable, upgradeable, and modular fashion.

However, a system model for complex topology and deep hierarchy is hard to obtain using model-based methods. In addition, efficient tests are difficult to develop for these complex systems [26]. Due to design complexity, knowledge about the system is typically "spread" over different engineers. Integrating and testing these diagnosis systems is a time-consuming, tedious and error-prone process.

Nevertheless, model-based diagnosis systems became popular during the last decade and they are still being utilized. For instance, [31] presents a comprehensive approach for model-based diagnosis, assuming that the system description is augmented with system structure.

In [27], Agilent Technologies presented a strategy to automate the debug process and allow for information sharing early in the design cycle between the hardware and software designers. Engineering information of a system model is documented during design time and it can be refined during product manufacturing time.

\section{Reasoning-Based Methods}

In reasoning-based diagnosis system, the fault isolation process is driven by an inference engine based on failure-symptoms correlation rules, expressed in the form 


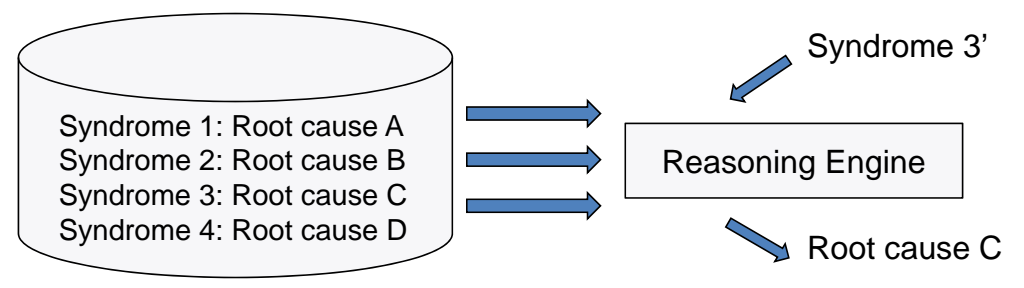

FigURE 1.5: Illustration of reasoning-based diagnosis methods.

of labels, as shown in Figure 1.5. In particular, reasoning-based systems are a special class of expert systems that make their decisions based on experience and past situations. They attempt to acquire relevant knowledge from past cases and previously used solutions to propose solutions for new problems. This goal is achieved through the learning of correlation patterns. When a problem is successfully solved, the solution may be used in dealing with subsequent problems.

However, reasoning-based systems require an application-specific model and a large dataset of previous cases to avoid incorrect learning. Furthermore, a broad set of different failure manifestations is necessary to avoid overfitting [32]. From a computational point of view, time inefficiency may make them unusable in real-time diagnosis scenarios [30].

Reasoning-based methods have been advocated for diagnosis through the use of machine-learning techniques. Artificial neural networks (ANNs) have been used for fault diagnosis in digital systems. For instance, in [33], authors built a diagnosing system using multi-layer ANNs for combinational circuits. Training of this diagnosis system is based on a database of identified potential faults, which are generated by inserting one fault in the circuit followed by simulation. In [4], Zhang et al. proposed a single-layer ANN for board-level diagnosis to achieve a significant improvement over previous ANN-based diagnosis methods. However, the training time becomes prohibitively long as the system complexity increases.

Butcher et al. in [34] presented a diagnosis system based on Bayesian networks. 
This diagnosis system can be either derived from domain knowledge or learned from actual tests, which are extracted from debugging and maintenance data. A statistically significant (large) dataset is required to reflect an appropriate relationship between faults and test outcomes. However, since different components (with nonuniform failure rates) are used in different production lines, and equipment used for testing can vary, training a diagnosis system using these aggregated data may lead to incorrect learning. Therefore, correction techniques based on conditional probabilities are used to alleviate data corruption [34].

In [35], authors proposed two diagnosis techniques based on fault dictionaries generated using fault signatures in the presence of failures. Dictionaries are used to train classifiers that can infer root causes from syndromes. One diagnosis system is designed as a fuzzy system to extract a set of if-then rules, while another is a radial-basis function system. In [36], Pous et al. proposed an approach based on distance metrics to overcome the limitation of the fault-dictionary approach. Since the faults used for building the dictionary may be different from the set of faults in the actual system under diagnosis, previously unrecognized faults may lead to incorrect prediction in actual diagnosis. Therefore, post-processing is used to reduce ambiguity in the dictionary-based diagnosis system; however, the resulting computation may come at a high cost.

Moreover, a significant amount of work has been carried out on model-update (i.e., refinement) solutions for reasoning-based diagnosis engines. An evaluation framework based on minimum-redundancy-maximum-relevance (mRMR) has been used to identify a set of syndromes with high discriminative ability [37]. However, it provides no diagnosis ability and requires additional algorithms for diagnosis. Researchers have developed a diagnosis agent to facilitate evaluation of the quality of diagnosis tests by leveraging information about board structure and test coverage [38]. This work aims to screen the tests with weak diagnosisability and increase 


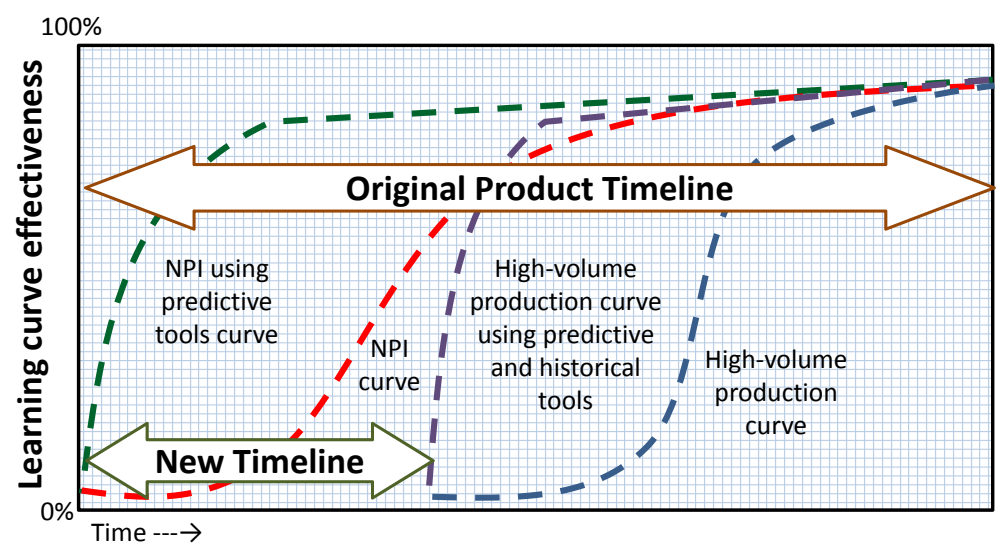

FiguRE 1.6: Illustration of new product ramp-up with and without reasoning-based tools $[2]$.

the diagnosisability at test-design stage, but it is not useful for volume production.

More reasoning-based diagnosis systems can be found in the literature [23, 25,33, 39-41], where machine learning techniques have been widely used.

\subsubsection{Automated Diagnosis System}

Reasoning-based methods are becoming popular today since detailed system models are not needed to construct the diagnosis system. Benefits of reasoning-based diagnosis system include:

- Reduced dependence on expert debug technicians.

- Faster ramp-up during the New Product Introduction (NPI) phase, since reasoning is available much sooner than the time needed by debug technicians to come up to speed (see Figure 1.6).

- Transfer of diagnostic knowledge from engineering to manufacturing.

A reasoning-based diagnosis system is embedded in the manufacturing test line, as shown in Figure 1.7. In today's manufacturing line, functional/system test is typically performed after products pass process test (AOI/AXI) and structural test 


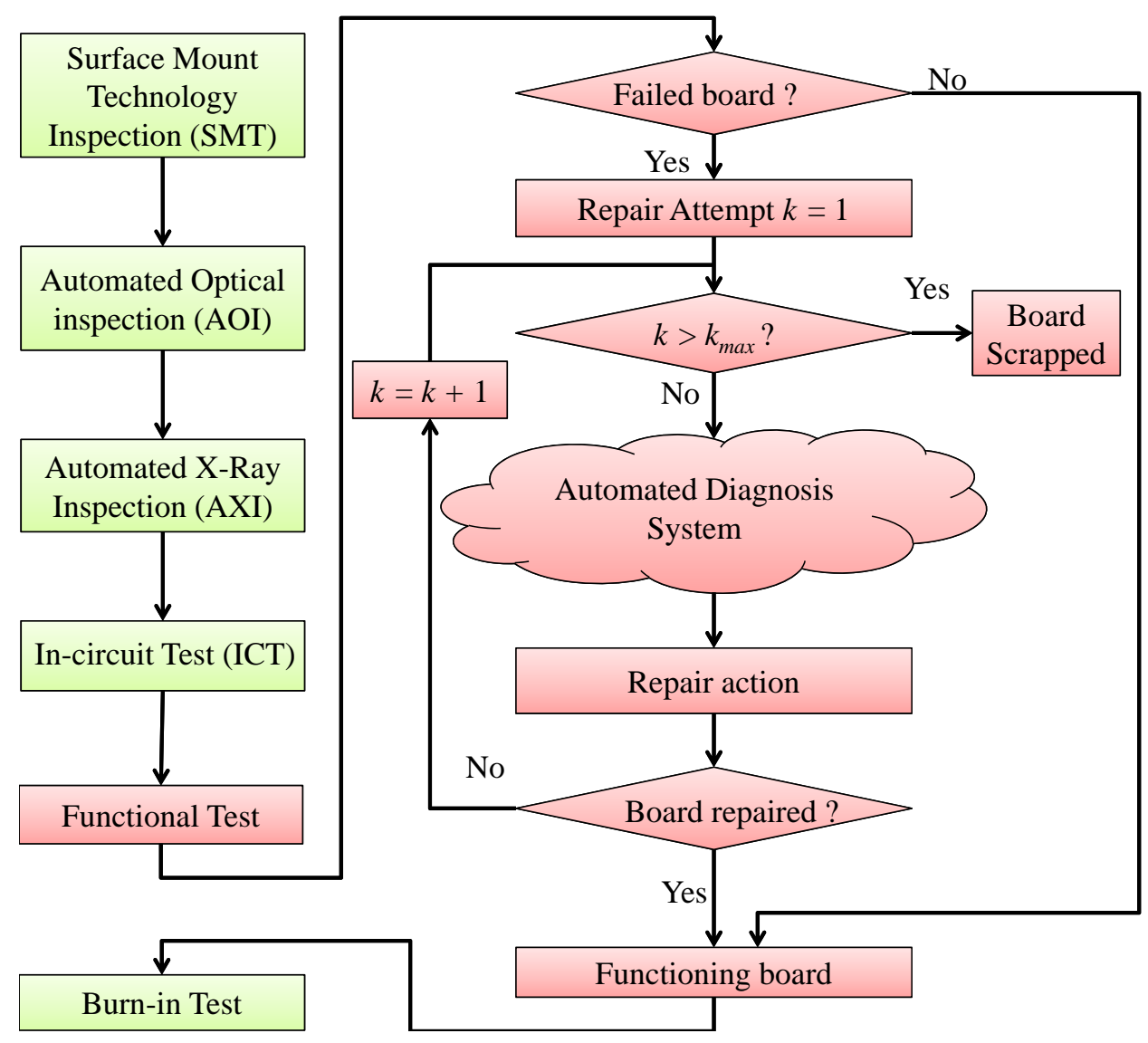

FIGURE 1.7: Flowchart for automated diagnosis.

(ICT and boundary scan test), in order to help isolation of the faults that escape structural tests. Functional test is designed to be close to the practical scenarios that can occur in the field. The conditions for each test are individually determined and set in order to mimic the particular practical scenario and not to influence the follow-up tests [42].

Once a failure is detected in the manufacturing line or in the field, the failed product is sent to the diagnosis department for repair. Typically, technicians run additional functional tests and measurements based on their personal experience. This process may take up to several days, and even several weeks if the board remains faulty after the component is replaced at the repair center [4]. In automated 
diagnosis system, test programs are loaded for diagnosing the faulty board. Based on the syndromes collected from the tests, the automated diagnosis system offers several suspicious root-cause candidates in a list, with the root cause of the most confidence on the top of the list. Repair action is executed on the suggested rootcause component. A repaired board is exercised again using test programs again to check functionality. If it continues to fail the test program, the faulty board will be taken back to the diagnosis system for another iteration of diagnosis. Note that a board has to be scrapped after a few unsuccessful repair attempts in a high-volume production environment. Compared to traditional diagnosis procedure executed by technician labors, which is time-consuming and no guarantee on the success of repair, the automated diagnosis system is more accurate and can be adaptive to the new knowledge. In addition, current diagnostic software is not able to accurately and rapidly locate root causes. After all, the reasoning-based automatic diagnostic system can facilitate board-level fault diagnosis and repair, and significantly reduce manufacturing cost.

The extraction of fault syndromes is critical for model training in a diagnosis system that targets functional failures. Design-for-Testability (DFT) support is required for this purpose. Without appropriate DFT support, the diagnosis system cannot generate meaningful syndromes for board diagnosis. The fault syndromes should provide a complete description of the failure, and the extracted syndromes for different actions should have sufficient diversity such that ambiguity can be eliminated in the eventual repair recommendations. Fault syndromes vary across products and tests. Taking network systems as an example, traffic tests are performed through route processors, interface modules, and port ASICs to ensure correct functionality and connectivity. Critical fault syndromes in a failed traffic test often consist of error counters, drop counters, interrupt bits, mismatch interfaces, ECC/CRC errors, etc. To extract these fault syndromes, log files are parsed using keywords and syndromes 


\begin{tabular}{l}
\hline SD587V_MASTERI:Interrupt Occur, SD587v_Masteri_Injt:0X00000028 \\
SD587_srx_int:0x00000002 \\
Interrupt caused by int_set[1] \\
SD587V_RBI_INT:0x00000020 \\
A packet was received with a framing error in RBI \\
$\ldots \ldots \ldots$ \\
LA0 Engine-error status................0x1000 000000 \\
LA1 Engine-error counter...............0x000f ffff \\
$\ldots \ldots .$. \\
Error Code: Ox26
\end{tabular}

Figure 1.8: A segment of the log file for a failed functional test.

are extracted using different descriptive-language scripts. For example, a segment of the $\log$ file for a failed traffic test is shown in Figure 1.8. The fault syndromes extracted are int_set[1] (interrupt bit), LA0 Engine 0x0000ffff (error counter) and 0x26 (error code). Each of these elements is considered to be one syndrome. According to the syntax of different log files, changes may be needed in parsing scripts. The repair action is often directly recorded in the database, e.g., "replacement is component U37".

The above diagnosis flow requires the availability of a sufficient amount of fail data that can be used for training. If such data is not available in sufficient quantity, faultinsertion test (FIT) can be used to extract fail data in an artificial manner [20,43]. FIT is a promising method for board/system reliability test and diagnosis coverage measurement. Due to the complexity of today's boards, random defects can occur, and unlike systematic defects that occur in a predictable manner and multiple times, random defects do not contribute in a meaningful way to system training. FIT verifies error detection by intentionally inserting wrong values at the pin/logic level. FITs can help mimic design corners and marginality, enrich the training set with rare events, and facilitate the timely release of a quality diagnosis system, and also provide feedback on the fault coverage of a complicated system. 


\subsection{Research Challenges and Motivation}

Printed-circuit boards (PCBs) and electronic systems are found in a wide range of applications, e.g., vehicles, medical equipment, data centers, and network communications. With an increase in system complexity, the need for automated effective diagnostic tools has become more acute. This is driven by economic scaling of test, limited board test access, reduced time-to-market, shorter life cycles of products, and environmental requirements. We describe some of these issues in more detail as below.

\section{Cost of Manufacturing Test}

With the advances in process technology, the fabrication cost per transistor continues to decrease, but the testing cost per transistor remains the same or even increases slightly. According to the international technology roadmap for semiconductors (ITRS) published in 2013 [44], the costs associated with testing and diagnosis is one of the highest contributors to manufacturing cost.

The cost of different levels of testing is often estimated using the rule of ten, as shown in Table 1.1 [1]. The table shows that if it costs $\$ 1$ to test an ASIC, the cost of locating the same defective ASIC when it is mounted on a board is about $\$ 10$; when the defective board is plugged into a system, the cost of identifying the faulty board and repairing the system is $\$ 100$. If the defective system is delivered to end users, the cost of recall and repair will go up to $\$ 1,000$. In 2007 , the hardware failure on the Xbox 360, the red ring of death, cost Microsoft more than $\$ 1$ Billion [45]. In 2008, Amazon lost $\$ 31,000 /$ min within 5 hours of service outage [46]. Therefore, it is necessary to diagnose failures at an early stage of assembly and provide practical diagnostic tools to reduce diagnosis and repair cost. 
Table 1.1: Rule of ten in test economics [1]

\begin{tabular}{|c|c|c|c|c|}
\hline Testing & $\begin{array}{c}\text { ASIC } \\
\text { Testing }\end{array}$ & $\begin{array}{c}\text { Board } \\
\text { Testing }\end{array}$ & $\begin{array}{c}\text { System } \\
\text { Testing }\end{array}$ & $\begin{array}{c}\text { Out in } \\
\text { Field }\end{array}$ \\
\hline Cost of Test & 1 & 10 & 100 & 1000 \\
\hline
\end{tabular}

\section{Reduction of Yield Loss}

The first-pass rate on current production lines is a matter of concern. For some complex telecommunication boards, only $60 \%$ to $70 \%$ boards pass functional tests the first time [4]. The number of failed boards waiting for diagnosis accumulates rapidly, especially in high-volume production. Circuit boards have to be scrapped, if failures are still detected after a few times of repair. This is a significant impediment to profit, since a complex telecommunication board costs up to thousands of dollars [47]. Therefore, efficient and accurate diagnosis methods are urgently needed.

\section{Accelerated Diagnosis}

In an increasingly competitive marketplace, time-to-market is critical for the success of a company. This is another driver for the use of automated test and diagnostic tools. Isolating the root cause of board-level functional failures usually requires an expert, with engineering skills in both hardware and software. First, depending on the complexity of the product, it can take several months (even years) to develop this level of expertise. During initial product ramp-up, this expertise is especially needed but often unavailable, hence diagnosis preparation time is often very long [2]. Next, isolating the root cause itself takes long time, since debug technicians needs to analyze the diagnosis log manually to identify the suspicious component. As the production volume increases and corresponding failing product volume increases, the diagnosis time inevitably increase dramatically. Therefore, an automated diagnosis engine is much urged for reducing the diagnosis time.

As discussed earlier in this chapter, a common scenario in industry is that of 
NTF. To address this problem, a diagnostic method is desired that can not only locate the faulty component on a malfunctioning board, but it can also narrow down the defective area inside the faulty component. The detailed failure information, e.g., failed test, failed sub-modules, failing cycles, is very important for component suppliers to troubleshoot and debug their manufacturing problems.

\section{Accommodate New Assembly Technology}

Environmental concerns, legislation, and market requirements are leading to increasing use of lead-free assembly technology in the electronics industry [48]. In 2006, the European Union Waste Electrical and Electronic Equipment Directive and Restriction of Hazardous Substances Directive (RoHS) came into effect to prohibit the intentional addition of lead to consumer electronics produced in the European Union. Quality and inspection of lead-free processes are emerging challenges in manufacturing test. The new assembly methods result in less access to boards for testing, debug and diagnosis. Changing package geometries are reducing the fault coverage that can be obtained using human visual inspection techniques. Some process imperfection and incorrect passive components may escape AXI, AOI and ICT test. Detection and diagnosis of such faults become new requirements in the functional test stage.

\section{Expand Industry Adoption and Use}

Another motivation for this research is that current application of automated boardlevel diagnostic tools is limited. Fault diagnosis has been an active area of recent research and development, but it still faces challenges related to accuracy, resolution and large data requirements. Over the past decades, automating fault diagnosis using artificial intelligence (AI) techniques has been a major research topic. There has been much progress, but industry acceptance, particularly in cost-sensitive seg-

ments, has not been high. The proposed research is expected to lead to generic and 
automated solutions for board-level fault diagnosis, which will provide quantifiable improvements over brute-force methods, trial-and-error manual repair, and previous proposed techniques based on expert systems. It is expected that the proposed automated solutions can be implemented and deployed in industrial manufacturing lines and debug departments.

\subsection{Outline of Dissertation}

The remainder of this dissertation is organized as follows.

Chapter 2 presents a fine-grained and adaptive diagnosis system for high-volume products. The propose diagnosis system is based on multi-kernel support vector machines (MK-SVMs) and incremental learning. The MK-SVM method leverages a linear combination of single kernels to achieve accurate faulty component classification based on the errors observed. The MK-SVMs thus generated can also be updated based on incremental learning, which allows the diagnosis system to quickly adapt to new error observations and provide even more accurate fault diagnosis.

Chapter 3 first reviews the use of artificial neural networks (ANNs). A data fusion technique is presented, namely majority-weight voting (MWV), to leverage multiple classifiers in our diagnosis system. The proposed MWV takes advantage of both ANNs and SVMs to provide an optimal repair-suggestion set.

Chapter 4 presents a decision tree-based diagnosis system to accelerate the diagnosis process. Considering that a large number of syndromes must be used in learning techniques to ensure diagnosis accuracy and effective board repair, the diagnosis cost can be prohibitively high due to the increase in diagnosis time and the complexity of syndrome collection/analysis. In the proposed adaptive decision tree-based diagnosis method, faulty components are classified according to the discriminative ability of the syndromes in DT training. The diagnosis procedure is constructed as a binary tree, with the most discriminative syndrome as root and final repair suggestions are 
available as the leaf nodes of the tree. The syndrome to be collected in the next step is determined based on the observations of syndromes collected thus far in the diagnosis procedure. The number of syndromes required for diagnosis can also be significantly reduced compared to the number of syndromes used for system training. Furthermore, an incremental version of DTs is used to facilitate online learning, so as to bridge the knowledge obtained at test-design stage with the knowledge gained during volume production.

Chapter 5 describe the design of preprocessing step in a diagnosis system to handle missing syndromes. Traditional diagnosis systems fail to provide appropriate repair suggestions when the diagnostic logs are fragmented and some error outcomes, or syndromes, are not available during diagnosis. Missing syndromes can be handled by using imputation methods. Several imputation methods are discussed and compared in terms of their efficiency in handling missing syndromes.

Chapter 6 presents an informative evaluation and enhancement framework for a diagnosis system. Learning in a diagnosis system requires a rich set of test items (syndromes) and a sizable database of faulty boards. An insufficient number of failed boards, ambiguous root-cause identification, and redundant or irrelevant syndromes can render the diagnosis system ineffective. The proposed evaluation and enhancement framework is based on information-theoretic concepts for guiding diagnosis systems using syndrome and root-cause analysis. Syndrome analysis based on subset selection provides a representative set of syndromes with minimum redundancy and maximum relevance. Root-cause analysis measures the discriminative ability of differentiating a given root cause from others. The metrics obtained from the proposed framework can also provide guidelines for test redesign to enhance diagnosis.

Chapter 7 presents a knowledge-discovery and a knowledge-transfer method for enhancing reasoning-based board-level functional fault diagnosis. Depending on the complexity of the product, it usually takes several months to accumulate an ade- 
quate database for training a reasoning-based diagnosis system. During the initial product ramp-up phase, reasoning-based diagnosis is not feasible for yield learning, since the required database is not available due to lack of volume. The proposed knowledge-discovery method and knowledge-transfer method can be used to facilitate board-level functional fault diagnosis. First, an analysis technique based on machine learning is used to discover knowledge from syndromes, which can be used for training a diagnosis engine. Second, knowledge from diagnosis engines used for earlier-generation products can be automatically transferred through root-cause mapping and syndrome mapping based on keywords and board-structure similarities.

Finally, Chapter 8 summarizes the contributions of the dissertation and identifies directions for future work. 


\section{2}

\section{Diagnosis Using Support-Vector Machines}

Diagnosis of functional failures at the board level is critical for improving product yield and reducing manufacturing cost. State-of-the-art board-level diagnostic software is unable to cope with high complexity and ever-increasing clock frequencies, and the identification of the root cause of failure on a board is a major problem today. Ambiguous or incorrect repair suggestions lead to long debug times and even wrong repair actions, which significantly increases the repair cost and adversely impacts yield.

In this chapter, we introduce a machine learning-based intelligent diagnosis system, which can automatically learn debug knowledge from empirical data and identify the most likely root cause of a new failed board. Using such a diagnosis system eliminates the difficulties involved in traditional knowledge acquisition. Fine-grained fault syndromes extracted from failure logs and the corresponding repair actions are used to train the system. Support-vector machines (SVMs) have been used in boardlevel diagnosis to provide accurate root-cause isolation. SVM-based diagnosis system can be rapidly trained and is scalable to large datasets. However, the SVM method used in prior work [3] was simplistic, relying on an arbitrarily chosen kernel function, 
and it was not adaptive to the availability of new data or test cases. We propose a diagnosis system based on multi-kernel support-vector machines (MK-SVMs) and incremental learning, which are used to tune the diagnosis system in an automatic manner. The MK-SVM method leverages a linear combination of single kernels to achieve accurate faulty component classification based on the errors observed. The MK-SVMs thus generated can also be updated based on incremental learning, which allows the diagnosis system to quickly adapt to new error observations and provide even more accurate fault diagnosis.

The remainder of this chapter is organized as follows. Section 2.1 reviews prior work and presents the problem statement. Section 2.2 reviews basic concepts in support-vector machines. Section 2.3 introduces multi-kernel-based SVMs, and describes how MK-SVMs can be extended for incremental learning, namely iMK-SVMs. Section 2.4 presents experimental results on diagnosis accuracy and training time for two industry boards and for synthetic data. These results are compared to diagnosis using single-kernel SVMs [3] and ANNs [4]. In addition, experimental results are presented for the diagnosis accuracy achieved using incremental learning. The high diagnosis accuracy, rapid training, and short diagnosis time highlight the benefits of the iMK-SVM-based reasoning system. Section 2.5 concludes the chapter.

\subsection{Problem Statement and Prior Work}

Field data and experience reports from repair technicians highlight many problems with diagnostic software currently in use, especially for functional tests that involve actual data in a real application. The diagnostic resolution offered by today's tools is limited to ASICs on the board. No repair guidance is provided for memory devices or passive components on the board. Diagnostic resolution is also poor in practice, multiple repair candidates are often listed and these candidates are not prioritized. Technicians are forced to run debug programs repeatedly and carry out physical 
probing in many places to identify the root cause of failures, a practice that significantly increases the debug and repair time. Based on past repair records, we have found the debug time for the functional test considered here to be as high as several weeks. The correctness of diagnosis, i.e., the probability of the actual failing component included in the list of suspects, is unacceptably low, and the root cause is seldom exclusively pinpointed.

In order to overcome the difficulties described above and provide accurate diagnostic results, we investigate intelligent diagnosis based on machine-learning algorithms. Machine learning, a branch of artificial intelligence, is focused on automatic learning from empirical data and making intelligent decisions. The debug knowledge can be automatically learned from history records (logs) using these techniques, e.g., artificial neural networks (ANNs) [4]. In ANN learning, we are given a set of training cases, which typically contain a set of error observations, referred to as syndromes. An ANN aims to automatically generate both the edge weights in the network and a transfer function that allow root-cause identification to be made on the basis of the syndromes. Due to its wide acceptance in the machine learning community and ease of interpretation, ANNs have been used for fault diagnosis [2,4]. However, ANNbased methods suffer from the inherent theoretical limitations of ANNs that tend to limit their accuracy [4]. Moreover, ANNs require large data sets for training, and large volumes of relevant data are not always available.

Recently, success in board-level functional fault diagnosis has also been reported using SVMs, which constitute a more advanced class of machine-learning techniques [3]. Even though SVMs were shown to be more effective than ANNs for a complex board in high-volume production [3], the increase in success ratio (diagnosis accuracy) was marginal. Moreover, the SVM method used in [3] was simplistic, relying on an arbitrarily chosen kernel function, and it was not adaptive to the availability of new data or test cases. 
In this chapter, we propose an adaptive, accurate, and efficient diagnostic system based on SVMs. An advantage of using machine learning is that it avoids the difficulties associated with knowledge acquisition and rule-base development required for expert systems $[3,4,49]$. Without the need to understand the complex functionality of boards, diagnostic systems based on machine learning are able to automatically derive and exploit knowledge from repair logs of previously documented cases. The proposed approach overcomes the limitations of single-kernel SVMs used in [3] by exploiting multi-kernel SVMs and incremental learning (iMK-SVM) to reduce complexity, achieve significantly higher diagnosis accuracy, and perform reasoning adaptively in realtime as new data becomes available. The kernel function in this approach is defined as a linear combination of different kernels. The proposed iMK-SVM-based diagnostic system is generic. Given a set of fault syndromes and the corresponding faulty components, the system can be rapidly trained and then used for fault diagnosis across different products. Results are presented for two industry boards, which are currently in production, and for which fail data has been gathered and used for training and evaluation.

\subsection{Diagnosis Using Support-Vector Machines}

A SVM is a supervised machine learning algorithm proposed by Vapnik in 1995 [50]. It has a number of theoretical and computational merits, for example, the simple geometrical interpretation of the margin, uniqueness of the solution, statistical robustness of the loss function, modularity of the kernel function, and overfitting control through the choice of a single regularization parameter. A brief introduction to SVMs is presented below. 


\subsubsection{Support-Vector Machines}

The goal of SVMs is to define an optimal separating hyperplane (OSH) to separate two classes. The vectors from the same class fall on the same side of the optimal separating hyperplane, and the distance from the closest vectors to the optimal separating hyperplane is the maximum among all the separating hyperplanes. An important and unique feature of this approach is that the solution is only based on those vectors that are the closest to the $\mathrm{OSH}$, calculated in the following way. let $\left(\boldsymbol{x}_{i}, y_{i}\right), i=1,2, \ldots, n$ be a set of training examples, and $\boldsymbol{x}_{i} \in \boldsymbol{R}^{d}, y_{i} \in\{-1,+1\}$. Figure 2.1 illustrates a 2-class SVM model. The vector $\boldsymbol{x}_{i}$ is considered as input, and $d$ is the dimensionality of the input vectors. Each input vector belongs to one of the two classes. One is labeled by $y=+1$; the other is labeled by $y=-1$. If the set can be linearly separated, there must be a hyperplane satisfying Formula 2.1:

$$
f(\boldsymbol{x})=\operatorname{sgn}\left(\boldsymbol{\omega}^{T} \boldsymbol{x}+b\right)
$$

where sgn refers to the sign of $\left(\boldsymbol{\omega}^{T} \boldsymbol{x}+b\right), \boldsymbol{\omega}$ is a $d$-dimensional vector, and $b$ is a scalar. Those vectors $\boldsymbol{x}_{i}$ for which $f\left(\boldsymbol{x}_{i}\right)$ is positive are placed in one class, while vectors $\boldsymbol{x}_{i}$ for which $f\left(\boldsymbol{x}_{i}\right)$ is negative are placed in another class. Based on [50], we define margin as twice the distance from the classifier to the closest data vector, namely the support vector. The margin is a measure of the ability to generate a classifier. The larger the margin is, the better is the generation of the classifier. SVMs maximize the margin between two classes.

Since the margin width equals $\frac{2}{\sqrt{\boldsymbol{\omega}^{T} \boldsymbol{\omega}}}$, the maximum-margin solution is found by solving the following minimization problem:

$$
\text { Minimize } \quad W=\frac{1}{2}\|\boldsymbol{\omega}\|^{p}+C \sum_{i}^{S} \xi_{i}
$$




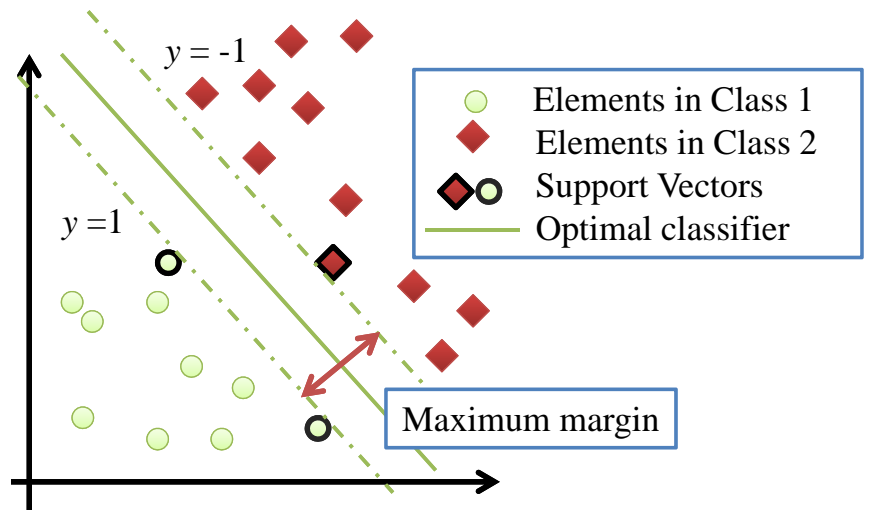

FIGURE 2.1: Illustration of a 2-class support-vector machine model.

Subject to

$$
\begin{gathered}
\left\|y_{i}\left(\boldsymbol{\omega} \cdot \boldsymbol{x}_{i}+b\right)\right\| \leq 1-\xi_{i}, \forall i \\
\xi_{i}>0, \forall i
\end{gathered}
$$

where slack variable $\xi_{i}$ is introduced to measure the degree of misclassification of data $\boldsymbol{x}_{i}$ and $C$ is the error penalty. We can tune $C$ to adjust the trained SVM model to be either over-fitting or under-fitting. The parameter $p$ is used for regularization of the weights in the SVM model. Most SVM solvers use standard regularization, i.e., $p=2$. Hence we assume $p=2$ in our work.

In order to solve the constrained optimization problem described in (2.2), a set of Lagrange multipliers $\alpha_{i}$, where $\alpha_{i} \geq 0$, is used. Each multiplier $\alpha_{i}$ corresponds to a constraint in (2.3) on the support vectors. The optimization problem from $(2.2)$ can now be expressed in its dual form

$$
\text { Minimize } \quad W_{1}=\frac{1}{2} \sum_{i, j=1}^{S} \alpha_{i} Q_{i j} \alpha_{j}-\sum_{i=1}^{S} \alpha_{i}+b \sum_{i=1}^{S} y_{i} \alpha_{i}
$$

where $Q_{i j}=y_{i} y_{j} K\left(\boldsymbol{x}_{i}, \boldsymbol{x}_{j}\right)$, and $K$ is the kernel function described in the next section. Additional mathematical details are omitted here but they can be found in [50]. The 
weights and offsets are as follows:

$$
\begin{gathered}
\boldsymbol{\omega}=\sum_{i=1}^{S} \alpha_{i} y_{i} \boldsymbol{x}_{i} \\
b=\frac{1}{N_{S}} \sum_{i=1}^{S}\left(y_{i}-\boldsymbol{\omega} \cdot \boldsymbol{x}_{i}\right)
\end{gathered}
$$

Originally SVMs were designed for linear binary classification problems. In practice, classification problems are not limited by two classes. In board-level fault diagnosis, the number of root cause candidates (classes) is typically in the range of a few hundreds. In [50], a modified design of SVMs was proposed in order to incorporate multi-class learning. Besides this, an alternative approach for handling a large number of classes is to combine multiple binary SVMs. "One against one" provides pairwise comparisons between classes. "One against all" compares a given class with all the other classes. According to a comparison study in [50], the accuracies of these methods are almost the same. Therefore, we choose the "one against all" in our problem, which has the lowest computation complexity.

\section{Demonstration of SVM-Based Diagnosis System}

To illustrate the SVM optimization procedure, consider the same hypothetical demonstration board with six cases in Section 2.2. We build an SVM model to identify faults for new cases. Let $x_{1}, x_{2}$ and $x_{3}$ be three syndromes. If the syndrome manifests itself, we record it as 1 , and 0 otherwise. The presentation of fault class is different from the that for ANNs training in Section 2.2. The board has two candidate root causes $\mathrm{A}$ and $\mathrm{B}$, and we encode them as $y=-1$ and $y=1$, respectively. In a real scenario, fault syndromes vary across products and tests. Here, we merge the syndromes and the known root causes into one matrix $\mathcal{A}^{\prime}=\left[\mathcal{B}^{\prime} \mid \mathcal{C}^{\prime}\right]$, where the left $\left(\mathcal{B}^{\prime}\right)$ side refers to syndromes, while the right side $\left(\mathcal{C}^{\prime}\right)$ refers to the corresponding 
fault classes. This matrix represents the training information for the SVM.

$$
\mathcal{A}^{\prime}=\left[\mathcal{B}^{\prime} \mid \mathcal{C}^{\prime}\right]=\left[\begin{array}{rrr:r}
1 & 1 & 0 & 1 \\
1 & 1 & 1 & 1 \\
1 & 1 & 0 & 1 \\
0 & 1 & 1 & -1 \\
0 & 0 & 1 & -1 \\
0 & 0 & 1 & -1
\end{array}\right]
$$

We obtain the Lagrange multipliers $\alpha_{1}=2.00 e^{-7}, \alpha_{2}=1.99, \alpha_{3}=1.99, \alpha_{4}=$ $1.99 e^{-7}$ by solving the optimization (cost) function from (2.5). We then get $\boldsymbol{\omega}_{1}=$ $1.99, \boldsymbol{\omega}_{2}=0, \boldsymbol{\omega}_{2}=0$ and $b=-1.00$ by solving Equation (2.6)-(2.7). Therefore, the classifier for determining the root cause for the given set is generated as follows:

$$
f(\boldsymbol{x})=\operatorname{sgn}\left(1.99 \cdot x_{1}+0 \cdot x_{2}+0 \cdot x_{3}-1.00\right)
$$

Next, suppose a new failing board is received and it has the syndrome $\left[\begin{array}{lll}1 & 1 & 0\end{array}\right]$, which corresponds to the first row (case) of $\mathcal{A}^{\prime}$ in Equation (2.8). The function $y$ is evaluated using Equation $(2.2)$, and since $\operatorname{sgn}(1.99 \cdot 1+0 \cdot 1+0 \cdot 0-1.00)$ is positive, $y=1$. Thus the root cause for this failing board is determined to be A. Suppose a second new failing board with syndrome $\left[\begin{array}{lll}0 & 1 & 0\end{array}\right]$ in received. In this case, the decision function evaluates to $y=-1$, hence we determine $\mathrm{B}$ to be the root cause in this case. For boards with the root cause of class A (B), we can replace the corresponding component A (B).

\subsubsection{SVM Diagnosis Flow}

The SVM-based diagnosis flow consists of four steps that are described in Figure 2.2. Generally speaking, a set of training data (fault syndromes and corresponding

repair actions) is first prepared, which is derived from the repair history in the manufacturing database. Then SVMs determine the OSH based on the training data. After the OSH is determined, the SVMs-based diagnostic system is ready to diagnose new cases. 


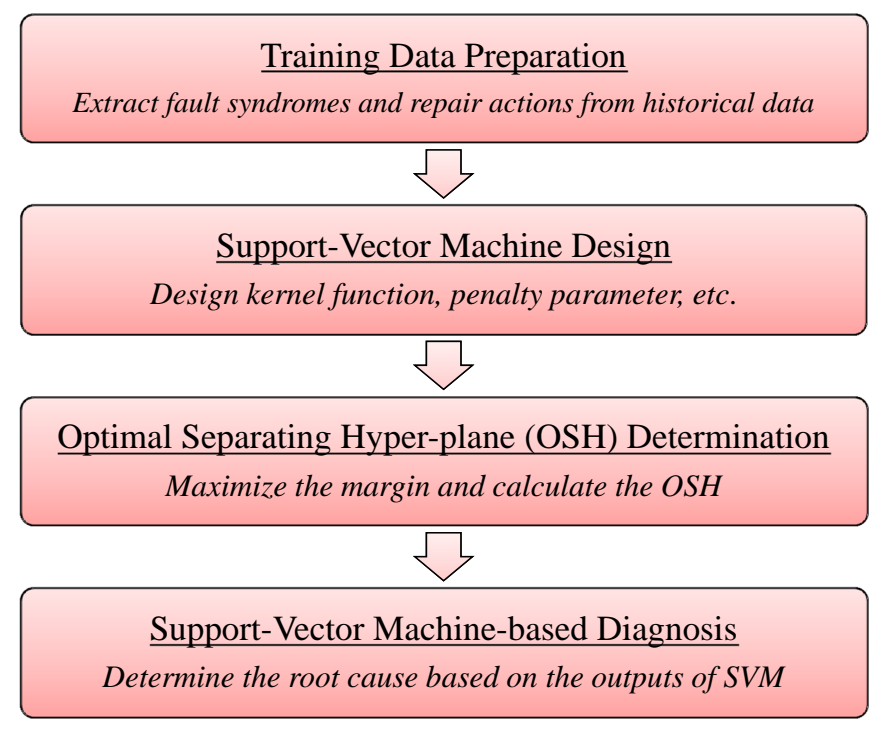

Figure 2.2: The diagnosis flow using SVMs [3].

Step 1: The data preparation step also follows the description in Section 2.1. The extracted syndromes and replaced components are used as inputs and outputs for the training of SVMs.

Step 2: Proper kernel function and penalty parameter are chosen to determine the SVMs training. The choice of these two parameters affects the performance of SVMs. However, there are no generic rules to select the best kernel and other parameters of SVMs for a specific problem. In this work, we determine the best design of SVMs in a heuristic way. According to extensive experimental results, we find that the SVMs with a linear kernel and a relatively large penalty parameter provide the highest diagnostic accuracy in the board-level fault diagnosis.

Step 3: The determination of the OSH can be considered as the training of SVMs. The OSH is determined by solving the quadratic optimization problem described in Equations (2.7) and (2.6). The values of $\boldsymbol{w}$ and $b$ can be calculated using Matlab. An open-source SVM toolbox is provided in [51]. The $\boldsymbol{w}$ and $b$ values are determined after training. 
Step 4: Given a new input vector, we can calculate the output of the SVM using the decision function in Equation (2.1). In the diagnosis step, we rank the output of all the SVMs, and select the component represented by the SVM with the largest output as the root cause.

\subsection{Multi-Kernel Support-Vector Machines and Incremental Learning}

Classical SVMs are efficient for linear classification, as discussed in Section 2.2. However, in many practical scenarios, including fault diagnosis, classical SVMs fail to find an optimal linear classifier for separating classes. Therefore, in such scenarios, SVMs must be extended to handle non-linear classification problems. One solution is to transform the problem to a higher-dimensional feature space through a non-linear mapping, also known as kernel, and the classifier is constructed in the new feature space. The advantage of this transformation is that it is not necessary to explicitly implement the transformation and to determine the separating hyperplane in a higher-dimensional feature space.

\subsubsection{Multi-Kernel Support-Vector Machines}

\section{Kernel}

In kernel-based transformation methods, the data representation is implicitly chosen through a kernel $K\left(\boldsymbol{x}_{i}, \boldsymbol{x}_{j}\right)$, where $\boldsymbol{x}_{i}$ and $\boldsymbol{x}_{j}$ are both input vectors in the lowerdimensional feature space. Figure 2.3 illustrates the transformation from a lowerdimension feature space to a high dimension feature space. The optimization problem from (2.5) can now be expressed as:

$$
\text { Minimize } \quad W_{2}=\frac{1}{2} \sum_{i, j=1}^{S} \alpha_{i} \alpha_{j} y_{i} y_{j} K\left(\boldsymbol{x}_{i}, \boldsymbol{x}_{j}\right)-\sum_{i=1}^{S} \alpha_{i}+b \sum_{i=1}^{S} y_{i} \alpha_{i}
$$


with similar constraint functions as those for non-kernel SVMs.

$$
b=\frac{1}{N_{S}} \sum_{i=1}^{S}\left(y_{i}-\sum_{j=1}^{S} \alpha_{j} y_{j} \cdot K\left(\boldsymbol{x}_{i}, \boldsymbol{x}_{j}\right)\right)
$$

The decision function (2.1) is now expressed as

$$
f(\boldsymbol{x})=\operatorname{sgn}\left(\sum_{i=1}^{S} \alpha_{i} y_{i} K\left(\boldsymbol{x}, \boldsymbol{x}_{i}\right)+b\right)
$$

The choice of kernel function is crucial for the success of an SVM-based model. There are several widely used kernel functions [50]:

- Homogeneous Polynomial Kernel: $K\left(\boldsymbol{x}_{i}, \boldsymbol{x}_{j}\right)=\left(\boldsymbol{x}_{i} \cdot \boldsymbol{x}_{j}\right)^{d}$, where $d \geq 1$. The linear kernel $(d=1)$ is regarded as a kernelized representation of linear SVMs.

- Polynomial kernel: $K\left(\boldsymbol{x}_{i}, \boldsymbol{x}_{j}\right)=\left(\boldsymbol{x}_{i} \cdot \boldsymbol{x}_{j}+1\right)^{d}$, where $d \geq 1$.

- Gaussian kernel: $K\left(\boldsymbol{x}_{i}, \boldsymbol{x}_{j}\right)=e^{-\gamma\left\|\boldsymbol{x}_{i}-\boldsymbol{x}_{j}\right\|^{2}}$, where $\gamma=\frac{1}{2 \sigma^{2}}$ and $\sigma$ can be interpreted as the standard deviation of a Gaussian distribution.

- Exponential kernel: $K\left(\boldsymbol{x}_{i}, \boldsymbol{x}_{j}\right)=e^{-\gamma\left\|\boldsymbol{x}_{i}-\boldsymbol{x}_{j}\right\|}$.

As an illustration, we apply kernelization methods to the example described in Section 2.2. Suppose that we choose a polynomial kernel with degree 2 and $K\left(\boldsymbol{x}_{i}, \boldsymbol{x}_{j}\right)=\left(\boldsymbol{x}_{i} \cdot \boldsymbol{x}_{j}+1\right)^{2}$. Given a new failing board under test with syndrome [ $\left.\begin{array}{lll}1 & 1 & 1\end{array}\right]$, the kernelized presentation of the syndrome is [0.91 0.640 .64$]$ and $f(\boldsymbol{x})$ is positive. Therefore, we can classify it as being in class A, i.e., the root cause is A. Suppose another new failing board is received with syndrome $\left[\begin{array}{lll}0 & 1 & 0\end{array}\right] ; f(\boldsymbol{x})$ is now negative. Hence, this board is classified as being in class B, i.e., the root cause of failure for this board is B. 


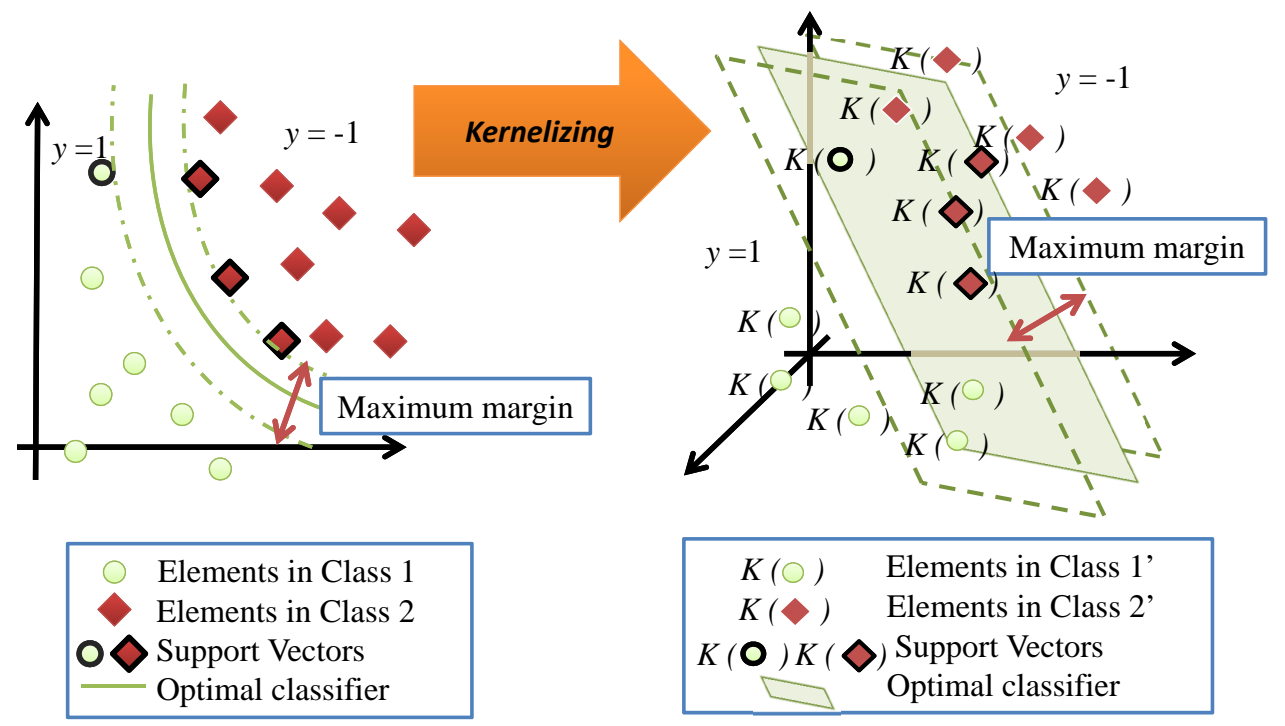

FiguRE 2.3: Illustration of kernelized support-vector machine model.

\section{Multi-Kernel Support-Vector Machines}

Recent applications in bioinformatics have shown that using multiple kernels instead of a single one can lead to better classification $[52,53]$. The key idea here is to represent the kernel $K\left(\boldsymbol{x}_{i}, \boldsymbol{x}_{j}\right)$ as a linear combination of $M$ basis kernels:

$$
K\left(\boldsymbol{x}_{i}, \boldsymbol{x}_{j}\right)=\sum_{k=1}^{M} \mu_{k} K_{k}\left(\boldsymbol{x}_{i}, \boldsymbol{x}_{j}\right)
$$

where $\mu_{k} \geq 0$ and $\sum_{k=1}^{M} \mu_{k}=1$. Each basis kernel $K_{k}$ can be one of the kernel types listed in Section 2.3. Thus the optimization problem can now be stated as:

$$
\begin{aligned}
\text { Minimize } \quad W_{3}= & \frac{1}{2} \sum_{i, j=1}^{S} \alpha_{i} \alpha_{j} y_{i} y_{j} k \sum_{k=1}^{M} \mu_{k} K_{k}\left(\boldsymbol{x}_{i}, \boldsymbol{x}_{j}\right) \\
& -\sum_{i=1}^{S} \alpha_{i}+b \sum_{i=1}^{S} y_{i} \alpha_{i}
\end{aligned}
$$




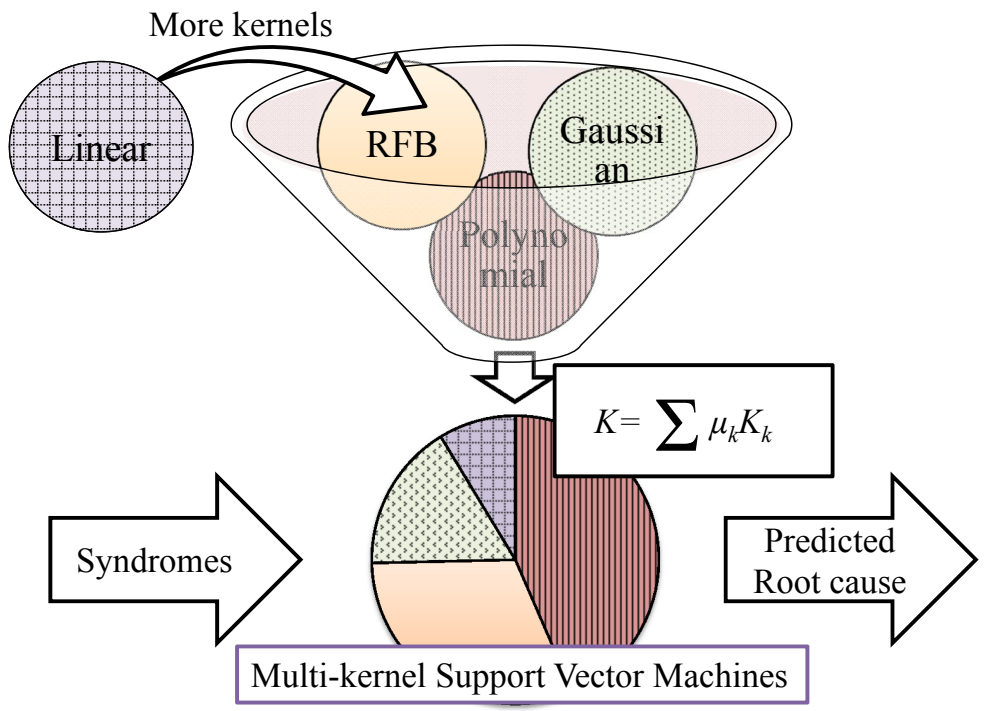

FIGURE 2.4: Illustration of training a multikernel SVM.

The optimization problem of equation (2.14) is solved using a reduced gradient method, the details of which are described in [54]. The training mechanism of a multikernel SVM-based diagnosis system is illustrated in Figure 2.4.

In previous work [3], the SVM-based diagnosis system leverages single kernels in a heuristic manner. However, due to the correlation between syndromes, we cannot arbitrarily determine a single kernel for each diagnosis system. A diagnosis system requires an adaptive kernel in order to achieve higher prediction accuracy. Such adaptation can extend from a single kernel to multiple kernels. Without knowledge of the exact kernel to be used, the weights of different kernels can be appropriately configured to fit the training data and generate better prediction for the diagnosis system.

\subsubsection{Incremental Learning}

Incremental learning can not only solve the SVM training problem for large scale data sets, but it can also facilitate online learning for SVMs as new data for failing boards and the corresponding repair outcomes become available. The use of multi- 
kernel SVMs increases computational complexity and diagnosis solutions take longer to converge. This problem can be tackled using incremental learning. By distributing the computational workload among different epochs during training, computational complexity can be reduced in terms of both computing time and memory allocation. Since SVM models can be determined on the basis of support vectors only, and the number of support vectors is typically very small compared to the number of training examples, SVMs can benefit from incremental learning through the compression of data from previous batches in the form of their support vectors. This approach to incremental learning with SVMs has been investigated in [55], where it has been shown that incrementally trained SVMs are as effective as their non-incrementally trained equivalents. Incremental SVMs can be described as the following optimization problem as an extension to (2.2):

$$
\text { Minimize } \quad W^{\diamond}=\frac{1}{2}\|\boldsymbol{\omega}\|^{2}+C\left(L \sum_{i \in S^{*}} \xi_{i}+\sum_{i \in S} \xi_{i}^{\prime}\right)
$$

with the same constraints (2.3) and (2.4). The parameter $S^{*}$ denotes the set of existing support vectors extracted from the previous SVM models, and $S$ is the new training set. As an optimization knob, the use of existing support vectors can be penalized by $L$ to model the fact that an erroneous decision made on the basis of previous support vectors is more costly than an error on a new example based on the current data. Incremental learning can be made more effective by combining it with multiple kernels, an approach that we refer to as iMLK-SVMs. A flowchart for this procedure is shown in Figure 2.5.

As an illustration, consider an existing diagnosis system given by Equation (2.1) and based on the input cases (boards) represented by the matrix $\mathcal{A}$ in Equation (2.8). The support vectors in the existing diagnosis system can be extracted as shown in $\mathcal{A}^{\prime}$. Suppose that we are given four new training boards (cases) indicated by the matrix 


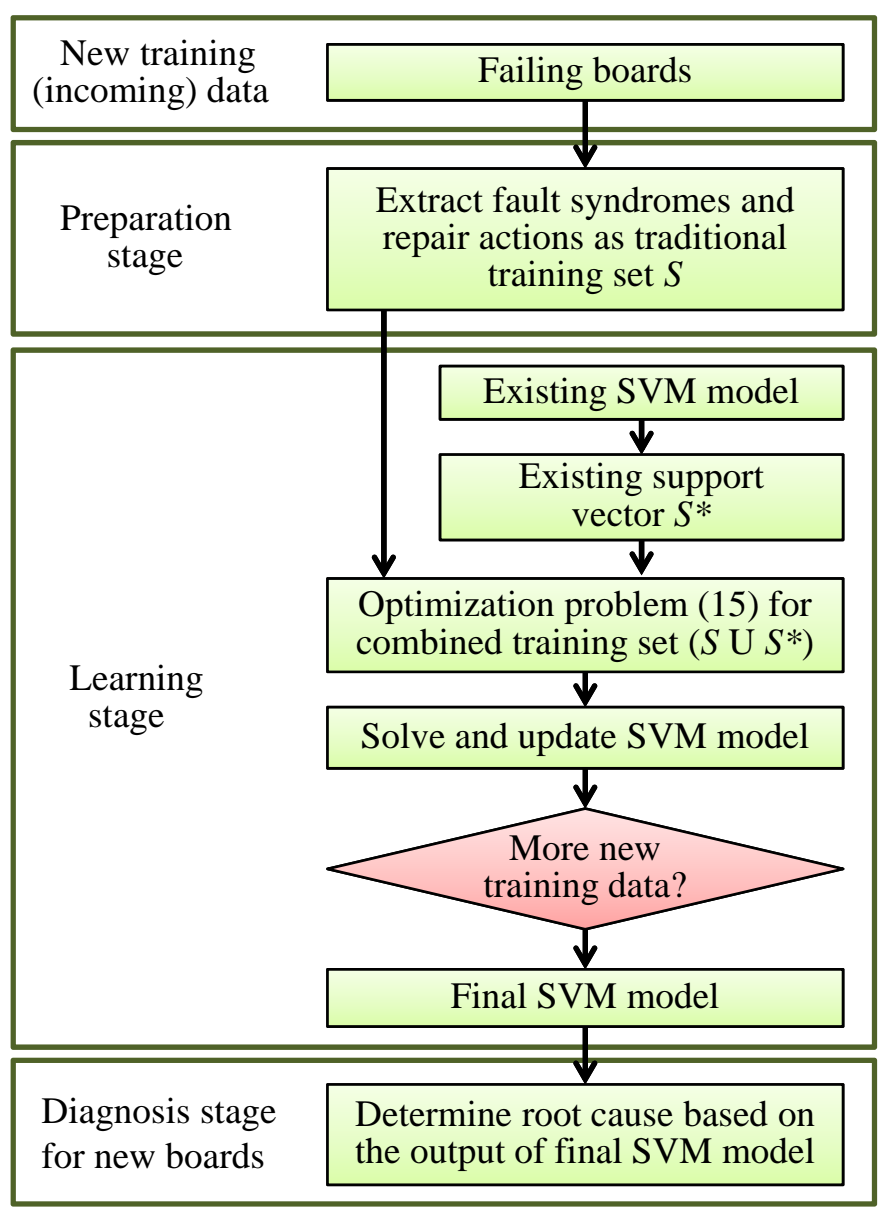

FIGURE 2.5: Illustration of the proposed iMK-SVM approach for fault diagnosis.

$\mathcal{N}$ in Equation (2.3.2) for incremental learning. The classifier (2.1) gives inconsistent root-cause identification - root cause A with the existing data but B with the new data. Therefore, we form a new data set $\mathcal{D}=\left[\mathcal{A}^{\prime} ; \mathcal{N}\right]$ as shown in Equation (2.3.2).

$$
\mathcal{A}^{\prime}=\left[\begin{array}{lll:r}
1 & 1 & 0 & 1 \\
1 & 1 & 1 & 1 \\
0 & 1 & 1 & -1 \\
0 & 0 & 1 & -1
\end{array}\right]
$$




$$
\begin{gathered}
\mathcal{N}=\left[\begin{array}{lll:r:r}
0 & 1 & 0 & 1 \\
0 & 1 & 0 & 1 \\
1 & 1 & 1 & -1 \\
1 & 0 & 1 & -1
\end{array}\right] \\
\mathcal{D}=\left[\begin{array}{c}
\mathcal{A}^{\prime} \\
\hdashline \mathcal{N}
\end{array}\right]=\left[\begin{array}{rrr:r}
1 & 1 & 0 & 1 \\
1 & 1 & 1 & 1 \\
0 & 1 & 1 & -1 \\
0 & 0 & 1 & -1 \\
\hdashline 0 & 1 & 0 & 1 \\
0 & 1 & 0 & 1 \\
1 & 1 & 1 & -1 \\
1 & 0 & 1 & -1
\end{array}\right]
\end{gathered}
$$

We obtain a unique solution to the new optimization problem from (2.15). We then get $\boldsymbol{\omega}_{1}=0, \boldsymbol{\omega}_{1}=0, \boldsymbol{\omega}_{1}=-2.00$ and $b=1.00$. The classifier is now updated from (2.1) to be:

$$
f(\boldsymbol{x})=\operatorname{sgn}\left(0 \cdot x_{1}+0 \cdot x_{2}-2.00 \cdot x_{3}+1.00\right)
$$

Next, we suppose that a new failing board is received and has the syndrome [ $\left[\begin{array}{lll}1 & 1 & 1\end{array}\right]$, which corresponds to the third row (case) of $\mathcal{N}$. The function $f(\boldsymbol{x})$ is now evaluated using Equation (2.16) and its value is negative, rather than the positive prediction based on the previous decision function of (2.1). Therefore, we can conclude that the failing board is in Class B, i.e., the root cause is B. Suppose another failing board is received with syndrome $\left[\begin{array}{lll}1 & 1 & 0\end{array}\right]$, which is the first test case from Section 2.2. In this case, the new decision function still evaluates $f(\boldsymbol{x})$ to be positive, hence we determine A to be the root cause for this board.

\subsection{Experiments and Results}

Experiments were performed on two industrial boards that are currently in highvolume production. Relevant information about the boards is provided in Table 
Table 2.1: Information about the industrial boards used for classification and the log data available.

\begin{tabular}{|c|c|c|}
\hline & Board 1 & Board 2 \\
\hline Number of syndromes & 546 & 375 \\
\hline $\begin{array}{c}\text { Number of repair } \\
\text { candidates (components) }\end{array}$ & 153 & 116 \\
\hline Number of boards & 1613 & 1351 \\
\hline
\end{tabular}

2.1. For training, a total of 1613 repaired boards are collected from the contract manufacturer's database for Board 1. A total of 546 fault syndromes are extracted from failure logs. The number of faulty components identified in the database for repair action is 153. For Board 2, a total of 1351 repaired boards are analyzed as training data. A total of 375 fault syndromes are extracted from failure logs. The number of faulty components for repair action is 116 .

The SVM algorithms are implemented using the Matlab 2010b toolbox. Multikernel SVMs are implemented using SimpleMKL [54]. Incremental learning is implemented using McpIncSVMs [56] and the SVM-KM toolbox [51]. As a comparison, the ANN method described in [4] has also been implemented using the Neural Network toolbox in Matlab 2010b [57]. Experiments were run on a 64-bit Windows system with $12 \mathrm{~GB}$ of RAM and quad-core Intel i7 processors running at $2.67 \mathrm{GHz}$. Diagnosis results were obtained for different designs of the SVMs, e.g., for various kernel functions. Moreover, diagnosis results were obtained to highlight the comparison between traditional artificial neural networks and the proposed multi-kernel SVMs. Incremental SVM-based diagnosis results were next compared with nonincremental SVM-based methods. Diagnosis results show that the training time is reduced significantly if we implement incremental learning in linear-kernel SVMs. Furthermore, iMK-SVM-based diagnosis results show high classification rates but low training time in each epoch, thereby providing a practical method for designing 
an adaptive diagnostic system.

In order to assess the performance of the classifier and its ability to accurately predict root cause of failure on a new board, we use a cross-validation method to partition the training set into $k$ groups, namely $k$-folder cross-validation [58]. Each group is regarded as a test case while all of the other cases are fed for training. In our work, we assess our model by using a special type of cross-validation method, namely leave-one-out (LOO), where the number of partitions $k$ is the same as the total number of cases. For example, for Board 2, each board instance is iteratively selected to be the test case. Classification models are based on the remaining 155 training cases. In LOO estimation, the total number of cases in the testing set is same as the total number of available successfully repaired boards.

To ensure real-time diagnosis and repair, we assume that we are allowed at most three attempts to replace the potential failing components. Success ratio (SR) is the ratio of the number of correctly diagnosed cases to the total number of cases in the testing set. We define $\mathrm{SR}_{1}$ as the success ratio corresponding to the case that the board is deemed to have been successfully repaired only when the actual faulty component is identified and placed at the top of the list of candidates. We also define $\mathrm{SR}_{2}\left(\mathrm{SR}_{3}\right)$ as the success ratio corresponding to the case that a board is deemed to have been successfully repaired if the actual faulty component is in the first two (three) positions in the list of candidates. In the last column in Table 2.2.(a), we can see that $\mathrm{SR}_{1}$ is $80.2 \%$. If three attempts are allowed in the repair process, $91.9 \%$ of the boards can be successfully repaired. These results are a significant improvement over other approaches reported recently in the literature [3,4]. The SR values for Board 2 are lower (Table 2.2.(b)). Nevertheless, tangible improvement is obtained over other methods, and the diagnosis accuracy is higher than that for methods currently used in production. The training data and evaluation methods will also continue to be used in the following chapters. 
Diagnosis Results Using The MK-SVM-Based Learning System

We use a combination of seven kernels in the multi-kernel machine, including linear kernel, Gaussian kernel ( $\sigma$ values of $0.5,1$, and 5), and polynomial kernel (degree $d=2,3,4)$. Diagnosis results are shown in Table 2.2.(a) for Board 1 and Table 2.2.(b) for Board 2. For Board 1, the $\mathrm{SR}_{1}$ for the multi-kernel SVM is $6 \%-18 \%$ higher than for the single kernel SVMs and the ANNs. When the $\mathrm{SR}_{3}$ is considered, the performance of multi-kernel SVMs is similar to that for the single-kernel SVMs. For Board 2, similar improvement of diagnosis accuracy by using multi-kernel SVM is obtained in Table 2.2.(b). The use of multi-kernel SVM technology leads to a considerable improvement in diagnosis accuracy, but the training time of the multikernel SVMs is higher compared to single-kernel SVMs and the ANN. For example, training MK-SVM for Board 1 requires up to an hour as compared to only tens of seconds using single kernel SVMs and ANNs. Since the training time depends on number of root causes, number of syndromes, number of cases and number of iterations required for convergence as described in Section 2.3, the training time of multi-kernel SVMs increases quadratically with the board complexity and number of failing boards that are returned for repair.

Diagnosis Results Using The Incremental SVM-Based Learning System

The use of incremental learning can help reduce the computational complexity of SVMs as described in Section 2.3.

We implemented linear SVM training on Board 1 and Board 2. The results of incremental learning for linear-kernel SVMs are shown in Figure 2.6 and Figure 2.8 for Board 1, and in Figure 2.7 and Figure 2.9 for Board 2. For example, we have a total of 813 training cases for Board 1 as described in Table 2.1. Initially, 800 training cases are randomly selected to build a base SVM model. In the second epoch, 100 more new training cases are randomly selected from the remaining pool. 
Table 2.2: Diagnosis results using ANN, SVMs with different kernel functions, and multi-kernel SVMs.

(a) Board 1

\begin{tabular}{|c|c|c|c|c|c|c|c|c|c|}
\hline & & \multicolumn{7}{|c|}{ SVM methods } \\
\cline { 4 - 10 } & ANN & $\begin{array}{c}\text { Linear } \\
\text { kernel }\end{array}$ & \multicolumn{2}{|c|}{ Polynomial kernel } & \multicolumn{2}{|c|}{ Gaussian kernel } & Multi- \\
\cline { 3 - 10 } & & $d=2$ & $d=3$ & $d=4$ & $\sigma=0.5$ & $\sigma=1$ & $\sigma=5$ & kernel \\
\hline $\mathrm{SR}_{1}$ & $67.9 \%$ & $73.2 \%$ & $74.4 \%$ & $72.3 \%$ & $74.9 \%$ & $62.6 \%$ & $65.1 \%$ & $62.6 \%$ & $80.2 \%$ \\
\hline $\mathrm{SR}_{2}$ & $78.1 \%$ & $80.4 \%$ & $82.2 \%$ & $81.2 \%$ & $82.7 \%$ & $74.0 \%$ & $76.3 \%$ & $74.5 \%$ & $85.4 \%$ \\
\hline $\mathrm{SR}_{3}$ & $84.4 \%$ & $88.2 \%$ & $91.9 \%$ & $90.4 \%$ & $91.9 \%$ & $79.2 \%$ & $82.5 \%$ & $79.3 \%$ & $91.9 \%$ \\
\hline $\begin{array}{c}\text { Training time } \\
\text { (seconds) }\end{array}$ & 71.2 & 43.2 & 45.2 & 41.1 & 42.0 & 49.9 & 50.3 & 50.1 & 5963 \\
\hline
\end{tabular}

(b) Board 2

\begin{tabular}{|c|c|c|c|c|c|c|c|c|c|}
\hline & \multirow{3}{*}{ ANN } & \multicolumn{8}{|c|}{ SVM methods } \\
\hline & & \multirow{2}{*}{$\begin{array}{l}\text { Linear } \\
\text { kernel }\end{array}$} & \multicolumn{3}{|c|}{ Polynomial kernel } & \multicolumn{3}{|c|}{ Gaussian kernel } & \multirow{2}{*}{$\begin{array}{l}\text { Multi- } \\
\text { kernel }\end{array}$} \\
\hline & & & $d=2$ & $d=3$ & $d=4$ & $\sigma=0.5$ & $\sigma=1$ & $\sigma=5$ & \\
\hline $\mathrm{SR}_{1}$ & $57.9 \%$ & $66.3 \%$ & $63.2 \%$ & $63.2 \%$ & $66.3 \%$ & $66.3 \%$ & $60.3 \%$ & $55.4 \%$ & $71.4 \%$ \\
\hline $\mathrm{SR}_{2}$ & $70.3 \%$ & $74.3 \%$ & $70.1 \%$ & $70.1 \%$ & $75.5 \%$ & $74.3 \%$ & $67.8 \%$ & $67.8 \%$ & $77.8 \%$ \\
\hline $\mathrm{SR}_{3}$ & $75.1 \%$ & $84.1 \%$ & $79.5 \%$ & $78.5 \%$ & $82.1 \%$ & $83.5 \%$ & $72.6 \%$ & $70.2 \%$ & $83.7 \%$ \\
\hline $\begin{array}{l}\text { Training time } \\
\text { (seconds) }\end{array}$ & 60.2 & 23.6 & 25.9 & 21.6 & 22.5 & 29.1 & 35.4 & 36.3 & 3891 \\
\hline
\end{tabular}

The existing SVM model is updated by using incremental learning to append these new training cases. A total of 100 more new training cases are added into SVM models in the next epoch and so forth. We also construct a non-incremental learning SVM from scratch in each epoch with the same number of training cases as in the corresponding incremental learning SVMs. Figure 2.6 shows that the training time for non-incremental learning SVMs increases linearly with the number of training cases, but the training time for incremental learning remains nearly constant in each epoch, even though the number of training cases increases. In the last epoch when 100 more training cases are appended to the existing SVM with 1500 training cases, the training time of SVMs using incremental learning is 8.27 seconds compared to 43.2 seconds using non-incremental learning. This quantifiable reduction in training time 


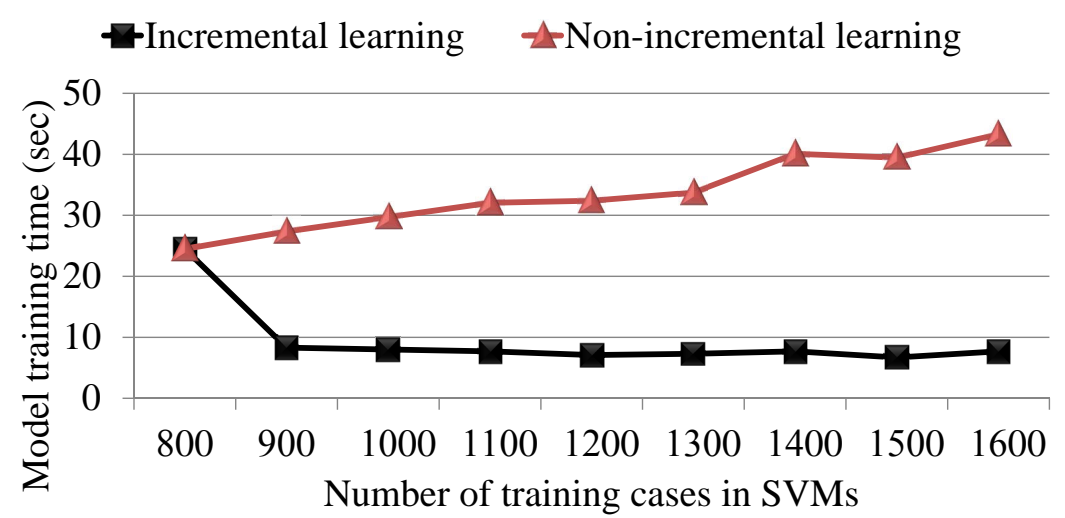

FiguRE 2.6: Comparison of training time in each epoch between incremental and non-incremental linear-kernel SVMs for Board 1.

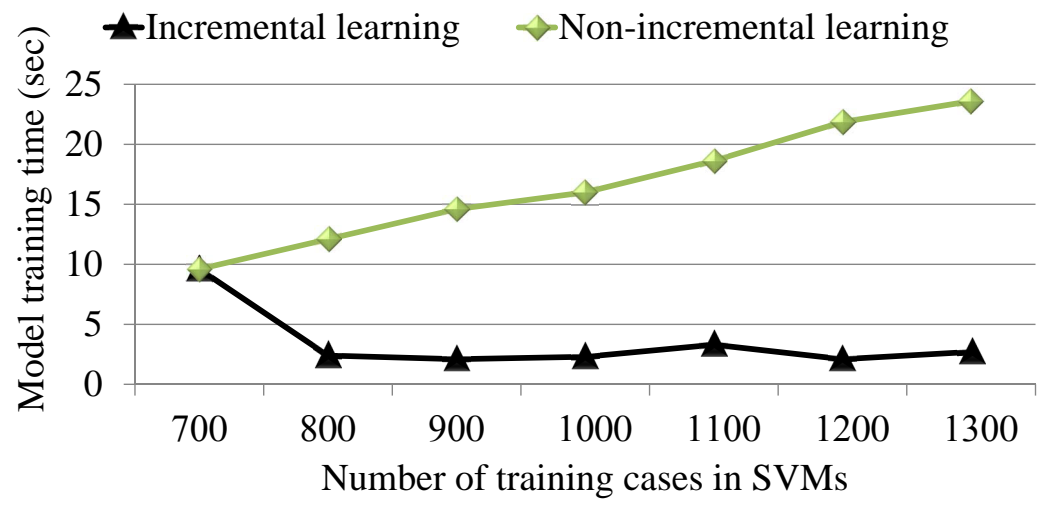

Figure 2.7: Comparison of training time in each epoch between incremental and non-incremental linear-kernel SVMs for Board 2.

using incremental learning can be a significant benefit if thousands of failing boards are returned for repair in high-volume manufacturing. Incremental learning also reduces computational complexity and memory required for training, as described in Section 2.3 .

The comparisons of $\mathrm{SR}_{1}$ between incremental learning and non-incremental learning are shown in Figure 2.8-2.9. We found that there is little difference in $\mathrm{SR}_{1}$ between incremental learning and non-incremental learning, thus non-incremental learning can be replaced by incremental learning with the similar success ratios, but 


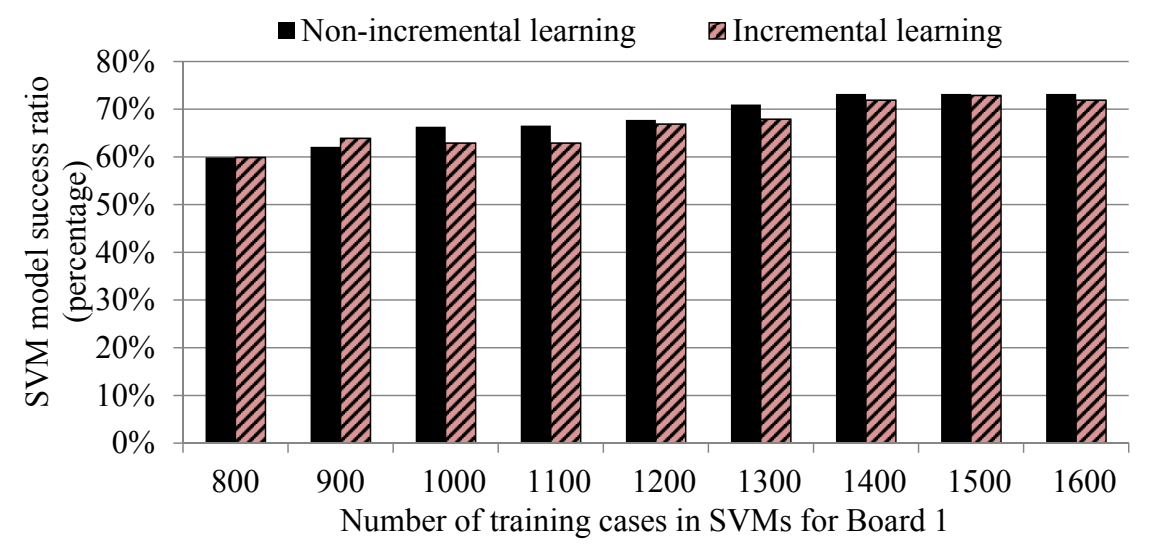

Figure 2.8: Comparison of $\mathrm{SR}_{1}$ between incremental and non-incremental linearkernel SVMs for Board 1.

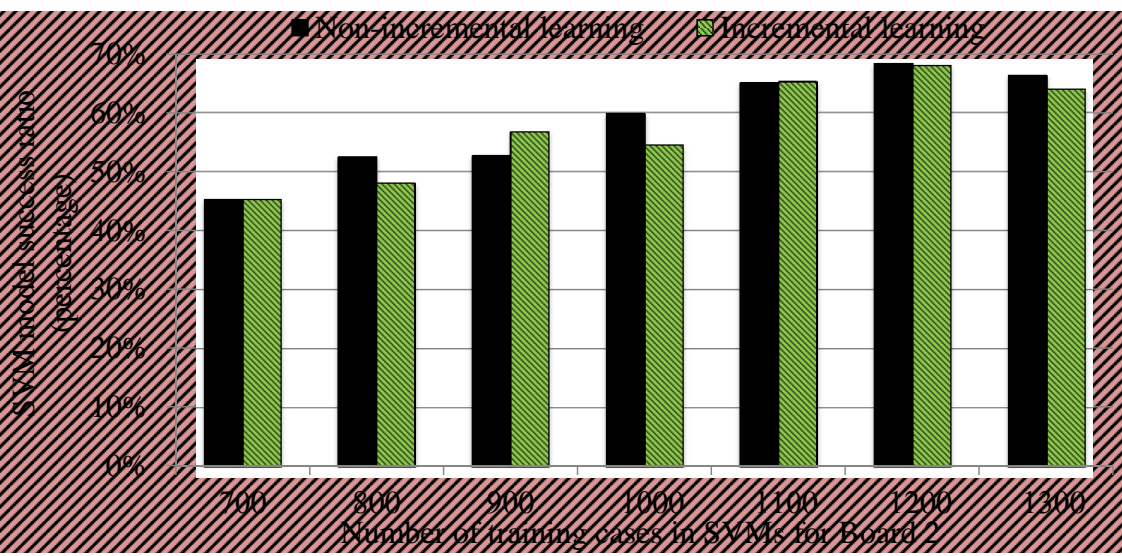

Figure 2.9: Comparison of $\mathrm{SR}_{1}$ between incremental and non-incremental linearkernel SVMs for Board 2.

the training time is reduced using incremental learning. The observations on $\mathrm{SR}_{2}$ and $\mathrm{SR}_{3}$ are the same as that on $\mathrm{SR}_{1}$. We also found that the $\mathrm{SR}_{1}$ increases when more failing boards are fed to the SVM for training. The diagnosis success ratio is strongly correlated to the size of the training set [3]. The $\mathrm{SR}_{1}$ of the linear-kernel SVMs increases from $59.5 \%$ with training cases of 800 to $73.2 \%$ with training cases of 1600 in Figure 2.8 for Board 1, and from 45.2\% in Table 2.2.(b) to $66.3 \%$ in Figure 2.9 for Board 2. The increasing trends of success ratios can also be found in $\mathrm{SR}_{2}$ and 


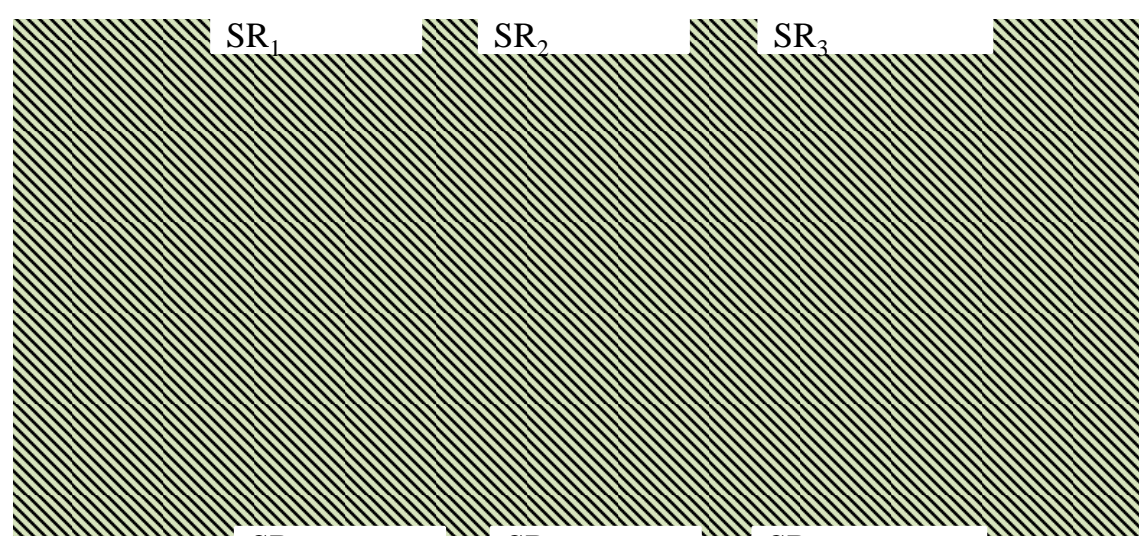

FiguRE 2.10: Success ratio of using incremental multi-kernel SVMs for Board 1.

$\mathrm{SR}_{3}$ for both Board 1 and Board 2.

In order to evaluate our results in a practical context, we consider what is typically carried out in a board manufacturing line. Most diagnosis and repair actions still rely on the technician's experience and trial-and-error methods. Current diagnostic software used in the production line from where we obtained the boards considers any component that exhibits error as a fault candidate, and no suggestions are provided regarding which component is more likely to be the root cause. Compared to the $\mathrm{SR}_{1}$ of the current diagnostic method, the $\mathrm{SR}_{1}$ for the proposed method is about two times higher, and significantly higher than even the $\mathrm{SR}_{3}$ of the currently used diagnostic method ${ }^{1}$. Based on the repair suggestions provided by the currently deployed method, the debug time for this particular functional test is as high as several weeks, which is clearly not feasible in practice. Debug efficiency is therefore expected to be improved considerably with the accurate repair suggestions provided by the proposed method. 


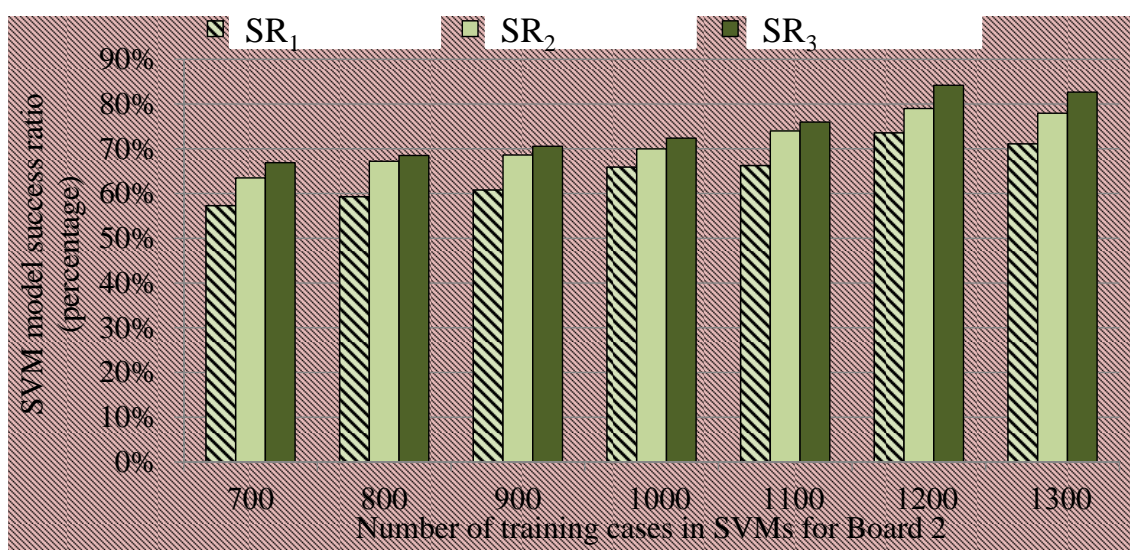

Figure 2.11: Success ratio of using incremental multi-kernel SVMs for Board 2.

Diagnosis Results Using The Incremental MK-SVM-Based Learning System

Incremental learning can also be applied to MK-SVM training. The success-ratio results are shown in Figure 2.10 for Board 1 and in Figure 2.11 for Board 2. When we increase the size of the training set, the success ratios of up to three attempts for both Board 1 and Board 2 both increase. This observation supports the positive correlation found in [3] between the number of failing boards available for training and the diagnosis accuracy on new boards. The $\mathrm{SR}_{1}, \mathrm{SR}_{2}$, and $\mathrm{SR}_{3}$ are 80.2\%, 85.4\%, and $91.9 \%$, respectively, when 1600 training cases are used in the iMK-SVM model for Board 1, and 71.4\%, 77.8\%, and 83.7\%, respectively, when 1300 training cases are used in the iMK-SVM model for Board 2.

The training time results are shown in Figure 2.12 for Board 1 and in Figure 2.13 for Board 2. The training time of incremental learning MK-SVMs in each epoch is much smaller than that for non-incremental learning MK-SVMs. The training time varies in each epoch for Board 1 due to the size of the training data and iterations needed for convergence, as described in Section 2.3. Due to the reduction of support vectors by using incremental learning techniques, the effect of iterations on the total

\footnotetext{
${ }^{1}$ Exact success ratio for the deployed system are not presented here in order to protect company
} confidential data. 


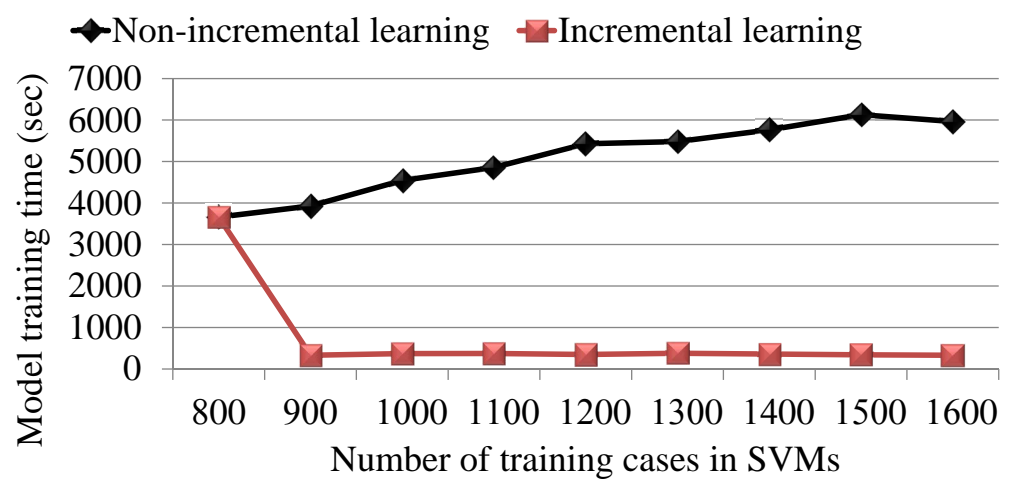

FIGURE 2.12: Comparison of training time in each epoch between using incremental and non-incremental multi-kernel SVMs for Board 1.

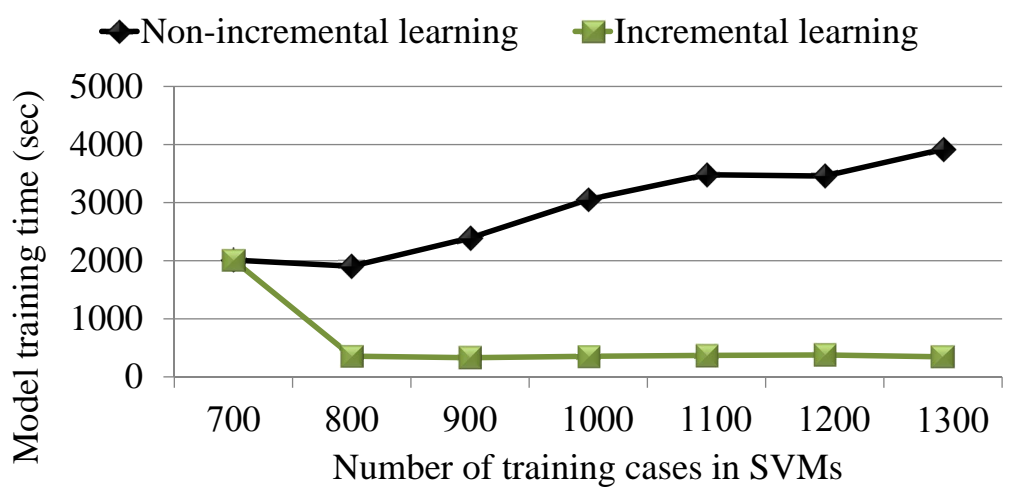

FiguRE 2.13: Comparison of training time in each epoch between using incremental and non-incremental multi-kernel SVMs for Board 2.

training time can also be reduced in iMK-SVMs, as shown in Figure 2.12.

The weights in iMK-SVMs training change in each epoch. For example, in Figure 2.14, we use a combination of 13 kernels in a multi-kernel machine, including the linear kernel, Gaussian kernel ( $\sigma$ values of $0.5,1,2,5,10,15,20$, and 50), polynomial kernel (degree $d=2,3$, and 4) and homogenous polynomial kernel (degree $d=2$ ). Only four kernels out of the total set of fourteen kernels contribute to the multikernel machine; these are the linear kernel, Gaussian kernel with $\sigma=2$, homogenous polynomial kernel (degree $d=2$ ), and polynomial kernel (degree $d=2$ ). The weights 


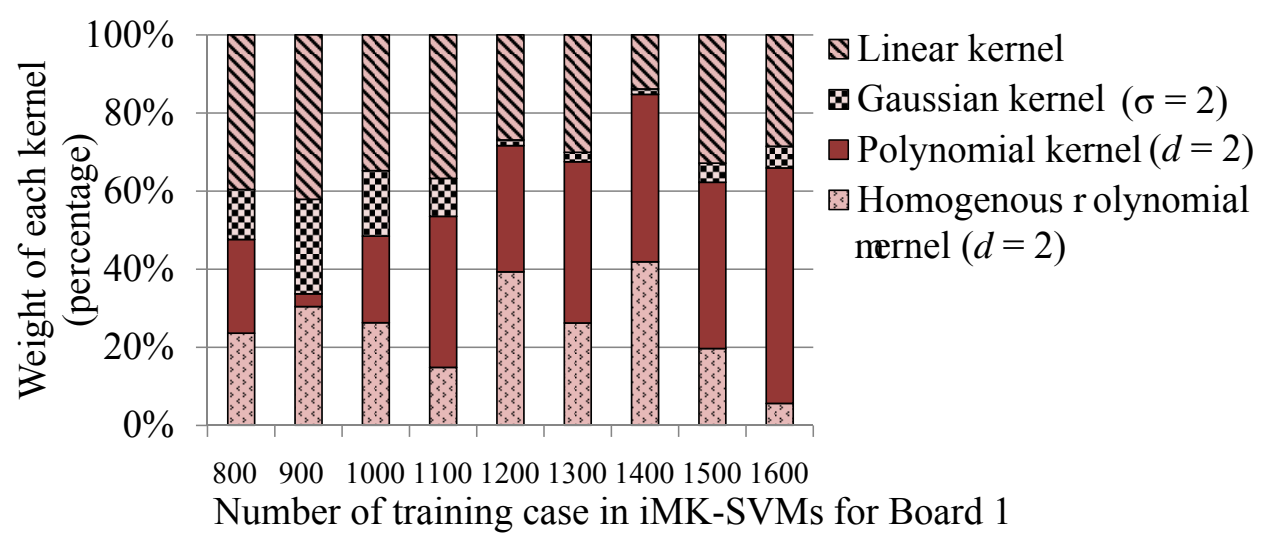

FIGURE 2.14: Illustration of the change in kernel weights in incremental multi-kernel SVMs for Board 1.

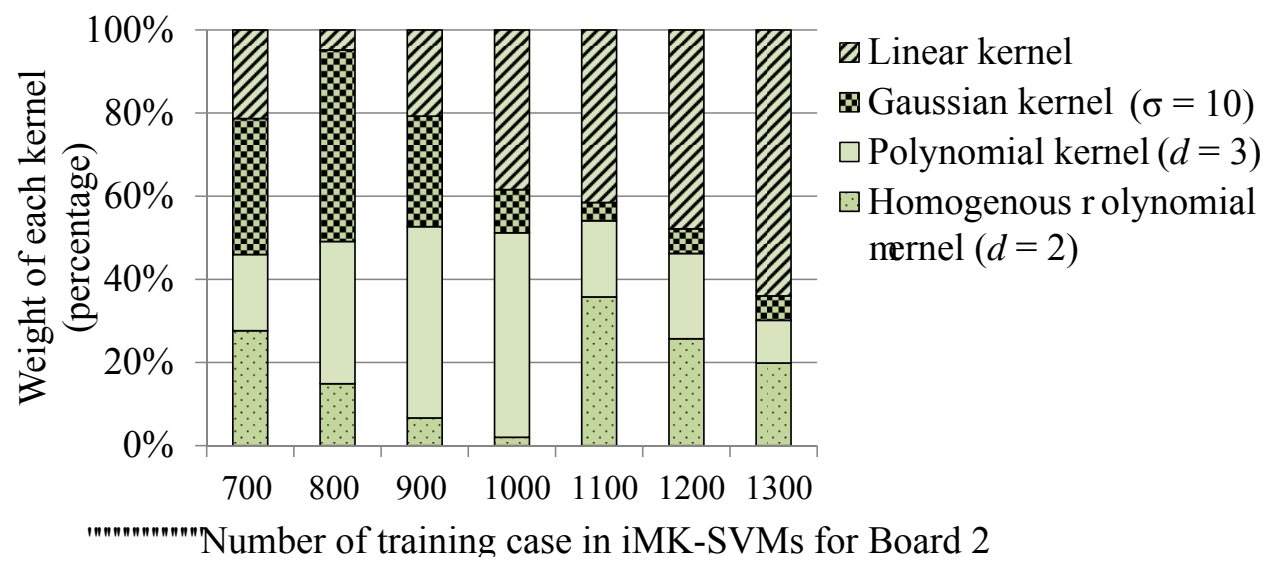

FigURE 2.15: Illustration of the change in kernel weights in incremental multi-kernel SVMs for Board 2.

of the remaining ten kernels are reduced to 0 in the optimized solution. In Figure 2.14, the weight of the Gaussian kernel with $\sigma=2$ is $12 \%$ and the weight of homogeneous polynomial kernel with degree $=2$ is $22 \%$ in the first epoch. When more cases are fed for training, the weights of these two kernels are gradually reduced to 0 . In the last epoch, only two kernels are left in the multi-kernel machine. And the dominate kernel is polynomial kernel with degree $=2(61 \%)$. Furthermore, kernel distribution is different for different board types. For Board 2, the linear kernel, the Gaussian 
kernel with $\sigma=5$, polynomial kernel with degree $=3$, and homogeneous polynomial kernel with degree $=2$ equally contribute to the multi-kernel in the first epoch. After three epochs, the weight of the homogeneous polynomial kernel is reduced to 0 . In the final epoch when 1300 cases are used for training, the weight of linear kernel is $65 \%$ and dominates the diagnosis system.

Since the optimal classifier solutions for different boards lead to different combinations of kernels, we cannot arbitrarily determine a best single kernel for all the boards. The use of iMK-SVMs can adaptively choose the most suitable kernel portfolio for different boards.

\subsection{Conclusions}

This chapter has presented a smart diagnosis system based on multi-kernel supportvector machines and incremental learning to locate the root cause of functional failures on modern circuit boards. The proposed multi-kernel SVMs method can generate an optimal kernel portfolio to achieve high diagnosis accuracy for board-level functional tests. The use of incremental learning allows the system to adaptively tune the kernel portfolio to achieve high diagnosis accuracy. System training time can also be reduced significantly using incremental learning. Two industrial boards, which are currently in high-volume production, and additional synthetic boards have been used to validate the effectiveness of the diagnosis method. Compared to baseline ANN and several single-kernel SVMs, multi-kernel SVMs show a considerable improvement in diagnostic accuracy based on functional patterns for a real application. 


\section{3}

\section{Diagnosis Using Multiple Classifiers and Majority-Weighted Voting}

In this chapter, we integrate a meta-learning technique, namely weighted-majority voting (WMV), in our diagnosis system. Multiple classifiers can be leveraged in a WMV-based diagnosis system to incorporate different sets of repair suggestions and form a single set of root-cause candidates. We review the usage of artificial neural networks (ANNs). The advantage of ANNs is its interpretation of the relationship between the syndromes and corresponding faulty components. A trained ANN model associates the output to the weighted inputs, which derives an intuition on the contribution of each input to the output. In addition, the advantage of SVMs, as described in Chapter 2, is that the solution provided by SVMs is globally optimal and unique, while ANNs suffer from multiple local minima. Both of these two methods can be rapidly trained and they are scalable to large datasets. The proposed WMV-based system uses weights to combine the repair suggestions provided by each machine in order to identify a single set of recommended repair suggestions. The proposed WMV system can leverage results from both ANNs and SVMs. 
The remainder of this chapter is organized as follows. Section 3.1 reviews the problem and states the contributions. Section 3.2 reviews the smart diagnosis system based on ANNs. Section 3.3 highlights the pros and cons of ANNs and SVMs used for functional fault diagnosis. Section 3.4 presents the WMV techniques to optimize the decision outputs of diagnosis by leveraging the repair component candidates provided by ANNs and SVMs. Section 3.5 presented experimental results on three industrial boards, including diagnosis accuracy and time cost. The results of ANNs and SVMs are presented separately based on their parameter settings. The results for the WMV technique are then presented to highlight the improvement in diagnosis accuracy. Section 3.6 concludes the chapter.

\subsection{Problem Statement and Chapter Contributions}

The diagnostic resolution offered by today's diagnostic tools is limited to chips on the board by using ATE. No repair guidance is provided for memory devices or passive components on the board. Technicians are forced to run debug programs repeatedly and carry out physical probing in many places to identify the root cause of failures, a practice that significantly increases the debug and repair time. Based on past repair records, we have found the debug time for the functional test considered here to be as high as several weeks. The correctness of diagnosis, i.e., the probability of the actual failing component included in the list of suspects, is unacceptably low, and the root cause is seldom exclusively pinpointed.

The proposed intelligent diagnosis based on machine learning algorithms can be used to overcome the difficulties described above and provide accurate diagnostic conclusions. Several machine learning technician has been used in board-level diagnosis, such as artificial neural network (ANNs), Baysian Neural Networks (BNNs), and Support-Vector Machines (SVMs) [3, 4, 40, 49, 59].

In [49], a Bayesian Inference (BI) machine is used to assist fault diagnosis. 
Bayesian inference offers a powerful probabilistic method for pattern analysis, classification, and decision making under uncertainty. Learning of BI does not depend on the structural test circuity, which eliminates the requirement of building standard BIST features for all devices on a board. Moreover, by learning the behavior of board/system under different faulty scenarios, the inference engine can automatically perform diagnosis without manual effort.

ANNs, which are inspired by the structure/functional aspects of biological neural networks, are used to model complex relationships between inputs and outputs, or to capture the statistical relationship between observed variables. In [33], a three-layer feed-forward network is trained with back propagation and is designed to target multiple faults in small combinational circuits. The test data are derived from a fault truth table, which is constructed by inserting random single stuck-at faults. The effectiveness of the method is validated only with small circuits that consists fewer than ten logic gates. In [60], the diagnosis of telephone exchange line cards using ANNs at British Telecom is described. A three-layer feed-forward network, with 77 input neurons and 8 output neurons, was constructed to solve the diagnosis problem in structural test. The inputs are measurements from in-circuit test (ICT). The outputs are fault candidates, which are 8 categories of the faulty components (e.g.,resistors, relays).

The problems targeted above are different from the functional diagnosis problem addressed in this thesis. In functional diagnosis, fault syndromes are extracted from a failure log, e.g., mismatched interface, error counter, etc. Therefore, fault syndromes are obtained from the results of functional test sequences, instead of measurements from ICT. Moreover, the diagnostic goal is to accurately locate the faulty component on the board rather than the category of the component. For example, hundreds of resistors can be soldered on a board. We obtain very limited diagnostic information, if we only determine that a resistor caused the failure, without pointing out the 
specific resistor.

In [4], a carefully crafted architecture of ANNs was used especially for boardlevel diagnosis. It can be rapidly trained and thus it can handle large datesets with thousands of inputs and outputs. It is initialized with the occurrence probability of fault syndromes, which significantly improves the diagnostic accuracy. It can easily interpret the relationship between the fault syndromes and repair action. The use of diagnosis system can raise the diagnosis accuracy up to $70 \%$.

In [3] and as described in Chapter 2, another fine-grained machine learning techniques has been explored for use in board-level diagnosis. The SVM algorithm is based on the statistical learning theory and the Vapnik-Chervonenkis (VC) dimension introduced in [50]. A Support Vector Machine (SVM) performs classification by constructing an optimal hyperplane that separates the data into two categories. Compared to ANNs, this new supervised learning method has a number of theoretical and computational merits. A significant advantage of SVMs is that the solution to an SVM is globally optimal and unique, while ANNs suffer from multiple local minima. we analyze the performance of SVMs with real manufacturing data.

However, the limitation of SVMs lie in selection of a proper kernel. In prior work [3], the best design of SVMs is determined by extensive simulation results. The proposed approach in Chapter 2 overcomes the limitations of single-kernel SVMs by exploiting multi-kernel SVMs and incremental learning (iMK-SVM) to reduce complexity, achieve significantly higher diagnosis accuracy, and perform reasoning adaptively in realtime as new data becomes available. The kernel function in this approach is defined as a linear combination of different kernels.

Moreover, single diagnosis system suffer from the limitation on its biased diagnosis and provides incorrect repair suggestion. In this chapter, we propose a diagnosis system based on weighted-majority voting. The proposed system uses weights to combine the repair suggestions provided by each machine in order to identify a single 


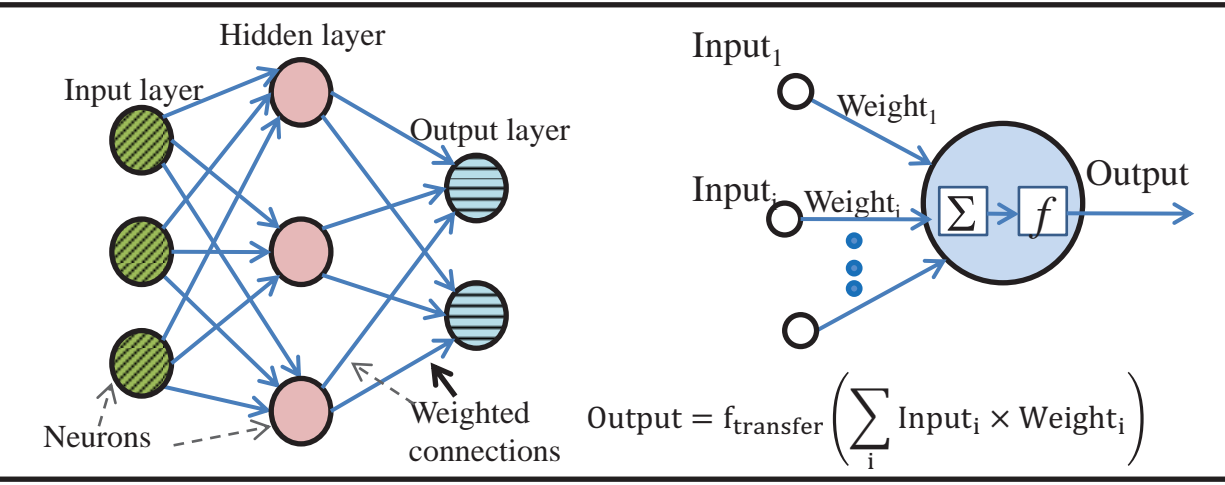

FigURE 3.1: A simple feed-forward neural network and the computation in a neuron $[4]$.

set of recommended repair suggestions. The proposed WMV-based diagnosis system can leverage results from both ANNs and SVMs.

\subsection{Artificial Neural Networks}

Artificial neural networks (ANNs) are widely used for pattern classification and related problems [61]. ANNs consist of neurons and weighted connections between neurons. Neurons are arranged in layers, and weighted connections link the neurons in different layers. A value is associated with each connection, referred to as weight, corresponding to the synaptic strength of neuron connections. The behavior of an ANN depends on both the weights and the input-output function, referred to as transfer function. This function typically falls into one of three categories, namely, linear, step, and sigmoid. A simple ANN and the computation in an artificial neuron is shown in Figure 3.1. Two basic network architectures are feed-forward and recurrent. In the feed-forward architecture, there is no feedback between layers as the network shown in Figure 3.1. In the recurrent architecture, there is feedback between layers, thus these networks can remember prior inputs. Before use, ANNs must learn from training examples. In supervised learning, we are given a set of example pairs, 


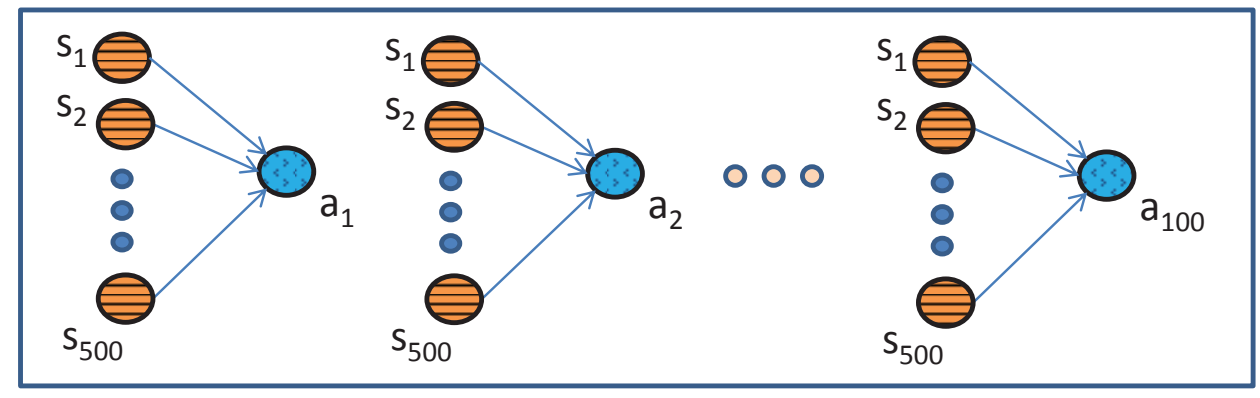

Figure 3.2: An illustration of the proposed ANN architecture [4].

and the aim is to find an appropriate function that matches the examples. Back propagation algorithm is widely used for training ANNs, which minimizes the the difference between actual outputs of ANNs and the desired values using gradient descent. When the difference is less than the pre-defined threshold, referred to as performance goal, the training is complete. The design and implementation of ANNs are supported by Matlab neural network toolbox [57]. A multi-layer feed-forward neural network can be easily implemented in Matlab.

ANNs are adopted to address the challenges involved in functional diagnosis at the board level. The intention is to develop an automated diagnosis tool that can learn from the historical repair data, involve less human effort, and provide accurate diagnostic guidance. The automated learning characteristic of ANNs fits this problem well. The ANN technique intelligently constructs the connections between fault syndromes and repair actions, without the need for a complete understanding of the complex functionality of a board.

Architecture of ANNs

In contrast to existing applications of ANNs to fault diagnosis, we use a group of single-layer networks to diagnose the root cause of board-level functional failures. The group size is equal to the number of known faulty components that have been replaced in the past (based on the available repair history). The initialization of 
weights is based on the occurrence probabilities of fault syndromes, instead of random initialization. For each single-layer network, the input neurons represent fault syndromes, and the single output neuron represents a component. Details on the extraction of fault syndromes and replaced components are presented in the next subsection. The input value is either 1 or 0 . A " 1 " implies that the fault syndrome appears in the log file; otherwise, it is a " 0 ". The desired (ideal) value at the output neuron is either "0" or " 1 ". A " 1 " means that the component represented by this network is the root cause of failure, and it should be replaced to repair the malfunctioning board; a "0" means that the corresponding component is not the root cause. The actual value at the output is a fraction between 0 and 1 , which can be viewed as the probability of the component being the root cause. A value closer to 1 implies that the component represented by the network is more likely to be the root cause, and vice versa. The proposed architecture is generic in the sense that it can be used for the functional diagnosis of various types of systems. For a different system, we only need to prepare a new set of training data for the ANNs, which can be easily achieved by updating the scripts used for syndrome extraction. An example of the architecture of ANNs with 500 syndromes and 100 actions is depicted in Figure 3.2. The training of the neural networks and diagnosis flow are described below.

\section{Demonstration of ANN-Based Diagnosis System}

To illustrate the training and testing procedure of the proposed ANN architecture, consider a hypothetical demonstration board with six cases that are used for training. We build ANN model to identify faults for new cases. All the test-outcome information is stored in a log file. The extracted syndromes and replaced components are used as inputs and outputs, respectively, for the training of the ANNs. Let $x_{1}, x_{2}$ and $x_{3}$ be three syndromes. If the syndrome manifests itself, we record it as 1 , and 0 otherwise. Let us suppose that the board has two candidate root causes A and B, 


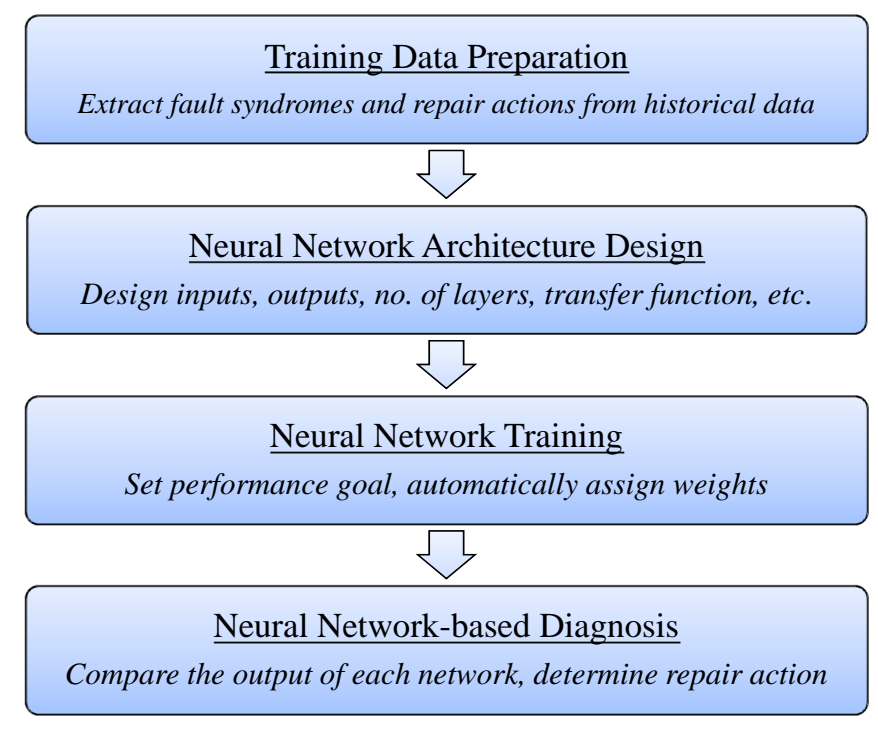

FIGURE 3.3: The diagnosis flow using neural networks [4].

and we encode them as $y=\left[\begin{array}{ll}1 & 0\end{array}\right]$ and $y=\left[\begin{array}{ll}0 & 1\end{array}\right]$, respectively. Note that the dimension of $y$ is as same as the number of candidate root causes. Each $y$ is a vector of only a single 1 and the remaining elements are 0s. In a real scenario, fault syndromes vary across products and tests. Here, we merge the syndromes and the known root causes into one matrix $\mathcal{A}=[\mathcal{B} \mid \mathcal{C}]$, where the left $(\mathcal{B})$ side refers to syndromes, while the right side $(\mathcal{C})$ refers to the corresponding fault classes. The resulting matrix, shown below in Equation (3.1), represents the training information for the ANNs.

$$
\mathcal{A}=[\mathcal{B} \mid \mathcal{C}]=\left[\begin{array}{lll:ll}
1 & 1 & 0 & 1 & 0 \\
1 & 1 & 1 & 1 & 0 \\
1 & 1 & 0 & 1 & 0 \\
0 & 1 & 1 & 0 & 1 \\
0 & 0 & 1 & 0 & 1 \\
0 & 0 & 1 & 0 & 1
\end{array}\right]
$$

We consider the transfer function to be purelin (linear transfer function) and the training function to be trainlm (LM-optimization). After training this one-layer system of ANNs, we obtain two weights vectors. Fault cause A is represented by the first weight vector $\boldsymbol{\omega}_{1}=\left[\boldsymbol{\omega}_{1-1} \boldsymbol{\omega}_{1-2} \boldsymbol{\omega}_{1-3}\right]$, where $\boldsymbol{\omega}_{1-1}=1.00, \boldsymbol{\omega}_{1-2}=3.26 e^{-7}$, 
$\boldsymbol{\omega}_{1-3}=8.51 e^{-8}$. Fault cause B is represented by the second weight vector $\boldsymbol{\omega}_{2}$, where $\boldsymbol{\omega}_{2-1}=-0.50, \boldsymbol{\omega}_{2-2}=0.25, \boldsymbol{\omega}_{2-3}=0.75$. Therefore, the vectors for determining the root cause is generated as follows:

$$
f(\boldsymbol{x})=\left(\begin{array}{c}
1.000 \cdot x_{1}+3.26 e^{-7} \cdot x_{2}+8.51 e^{-8} \cdot x_{3} \\
-0.50 \cdot x_{1}+0.25 \cdot x_{2}+0.75 \cdot x_{3}
\end{array}\right)
$$

Next, suppose a new failing board is received and it has the syndrome $\left[\begin{array}{lll}1 & 1 & 0\end{array}\right]$, which corresponds to the first row (case) of $\mathcal{A}$ in Equation (3.1). The function $y$ is evaluated using Equation $(3.2)$, and since $\left(1.000 \cdot 1+3.26 e^{-7} \cdot 1+8.51 e^{-8} \cdot 0\right)=1.00$, while $(-0.50 \cdot 1+0.25 \cdot 1+0.75 \cdot 0)=-0.25$, so $y=\left[\begin{array}{ll}1 & 0\end{array}\right]$. Thus the root cause for this failing board is determined to be A. Suppose a second new failing board with syndrome $\left[\begin{array}{lll}0 & 1 & 0\end{array}\right]$ in received. In this case, the decision function evaluates to $y=[0$ 1], hence we determine $B$ to be the root cause in this case. For boards with the root cause of class A (B), we can replace the corresponding component A (B).

\subsection{Comparison between ANNs and SVMs}

The development of ANNs followed a heuristic path, with applications and extensive experimentation preceding theory [4]. In contrast, the development of SVMs involved sound statistical learning theory, implementation, and experiments. The fundamental difference between the two approaches is that ANNs minimize empirical risk (misclassifications in the training set), but SVMs minimize the risk of misclassifying cases of the test set. Therefore, SVMs are less prone to overfitting and often outperform ANNs in practice.

ANNs use a backpropagation learning algorithm to search for the minimum of the error function (mean squared error) in the weight space. The combination of weights, which minimizes the error function, is considered to be a solution of the learning problem. However, the ANN solution obtained using a backpropagation 
algorithm can only converge to a local minimum. In contrast, the solution to an SVM is global and unique, because SVMs are formulated as a convex quadratic optimization problem.

In addition, the computational complexity of SVMs does not depend on the dimensionality of the input space. SVMs do not attempt to control model complexity by keeping the dimension of the input vectors small. In order to linearly separate the cases, SVMs sometimes transfer the input space to higher dimensional feature space. The model complexity of SVMs is automatically determined in the quadratic

programming procedure by selecting the support vectors. One more advantage of SVMs is the simple geometric interpretation, which allows us to easily maximize the margin.

\subsection{Diagnosis Using Weighted-Majority Voting}

Section 2.2 and Section 3.2 described diagnosis systems based on two machine learning methods, namely ANNs and SVMs. We regard these two machine learning methods as two expert systems. Each system provides its own repair suggestions based on the separate diagnosis techniques. Since we cannot predict which system is more accurate in general, we expect diagnosis with higher resolution to be achieved through a combination of the two systems.

\subsubsection{Weighted-Majority Voting}

Weighted-majority voting (WMV) is a meta-learning reasoning procedure [62]. The first type of majority voting refers to the decision when all experts agree on the same output (unanimous voting). The second type of majority voting refers to the decision when at least one more than half the number of experts agree on the same output (simple majority voting). The third majority voting approach is called weightedmajority voting. If we have evidence that certain experts are more qualified than 


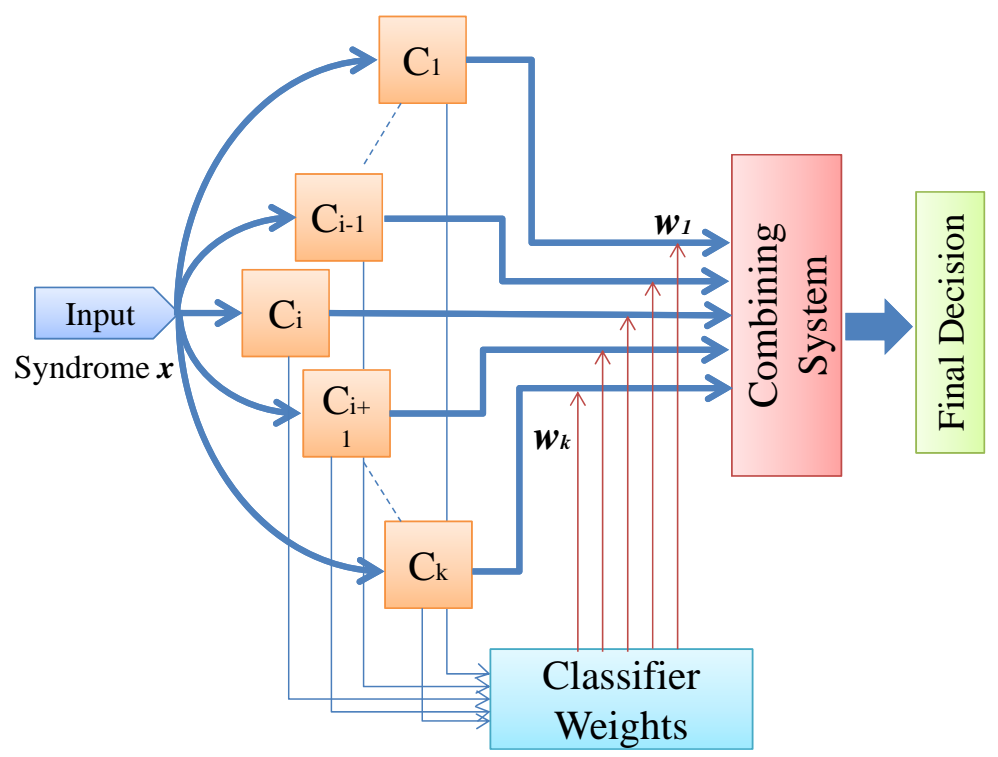

FIGURE 3.4: Illustration of weighted-majority voting.

others, weighting the decisions of those qualified experts more heavily may further improve the overall performance than that obtained by plurality voting. Let us denote the decision of classifier $C_{i}$ on class $l_{j}$ as $d_{i, j}$, such that $d_{i, j}$ is 1 if Classifier $C_{i}$ selects $l_{j}$ and 0 otherwise. Furthermore, assume that we have a way of estimating the performance of each classifier, and we assign weight $w_{i}$ to classifier $C_{i}$ in proportion to its estimated performance. According to this notation, the classifiers whose decision are combined through weighted majority voting will choose class $J$ if

$$
\sum_{i=1}^{I} w_{i} d_{i, J} \geq \max _{j=1}^{C}\left\{\sum_{i=1}^{I} w_{i} d_{i, j}\right\}
$$

i.e., if the total weighted vote received by $l_{j}$ is greater than or equal to the total vote received by any other class. Weighted-majority voting is illustrated in Figure 3.4.

The weight $w_{i}$ is associated with the estimated performance of classifier $C_{i}$. If we can predict which classifier is more efficient, we can assign the highest weight to this classifier, or even use this classifier alone. In the absence of this knowledge, a plausible strategy is to use the performance of a classifier on a separate validation dataset, or 
even its performance on the training dataset, as an estimate of that classifier's future performance. The weighting strategy is to assign weights as follows:

$$
w_{i}=\log _{2}\left(1 / \beta_{i}\right)
$$

where $\beta_{i}=\varepsilon_{i} /\left(1-\varepsilon_{i}\right)$, and $\varepsilon_{i}$ is the weighted training error of Classifier $C_{i}$. The training error $\varepsilon_{i}$ indicates the fitting degree of Classifier $C_{i}$ to the training data. This estimation has been validated in [62]. For easier interpretation, we can normalize these weights so that they sum up to 1; however, normalization does not change the outcome of weighted majority voting.

\subsubsection{Demonstration of WMV-Based Diagnosis System}

Consider the top-three repair suggestions provided by SVMs and ANNs. Suppose the repair suggestions provided by SVMs are $s_{1}, s_{2}$, and $s_{3}$, and that by ANNs are $a_{1}, a_{2}$, and $a_{3}$. The classifier weights $w_{s v m}$ and $w_{a n n}$ can be immediately calculated based on their empirical training errors. We also assign parameters $m_{1}, m_{2}$, and $m_{3}$ (refer to the WMV vector weights) to the corresponding ranks of the three repair suggestions.

Consider a hypothetical demonstration board and suppose that the board has three candidate root causes A, B, and C. After the SVM and ANN classifiers are trained on the same training set, suppose we have $\varepsilon_{\text {svm }}=0.07$ and $\varepsilon_{\text {ann }}=0.10$. Therefore, we can calculate the weights using Equation (3.4). We obtain the classifier weights $w_{\text {svm }}=3.73$ and $w_{\text {ann }}=3.10$. We also arbitrarily define $m_{1}=4, m_{2}=2$ and $m_{3}=1$ for demonstration purposes.

Suppose we use SVMs to get the repair suggestions A, B, and C in descending priority order. The repair suggestions provided by ANNs are ordered as A, C, and B. We have an intuition of the final decision rank that the root cause A should be the first attempt because both classifiers reach the same root cause. Root cause B 
should be the second attempt since SVMs are more reliable than ANNs due to the lower training error. Finally, root cause C should be considered as the third attempt.

We next apply WMV on the repair suggestions provided by SVMs and ANNs. According to Equation (3.3), the majority-voting vector $[\mathrm{A}, \mathrm{B}, \mathrm{C}]$ is $\left[\left(m_{1} \cdot w_{\text {svm }}+\right.\right.$ $\left.\left.m_{1} \cdot w_{\text {ann }}\right)\left(m_{2} \cdot w_{\text {svm }}+m_{3} \cdot w_{\text {ann }}\right)\left(m_{3} \cdot w_{\text {svm }}+m_{2} \cdot w_{\text {ann }}\right)\right]=[27.3,10.6,9.9]$. This rank is also $\mathrm{A}, \mathrm{B}$, and $\mathrm{C}$, which supports our intuition.

Consider another example with four candidate root causes A, B, C, and D. Suppose SVM-based diagnosis provides repair suggestions $\mathrm{A}, \mathrm{B}$, and $\mathrm{C}$ in descending priority order, but the actual root cause is C. Without additional classifiers, two incorrect repair actions will be taken before the board is successfully repaired. Next, we use WMV with additional input from the ANN model that provides a ranked order of candidates C, B, D. Suppose we have the same WMV vector weights as in the previous example, and the ANN classifier is weaker than the SVM classifier, where $\varepsilon_{\text {svm }}=0.07$ and $\varepsilon_{a n n}=0.10$. Therefore, we obtain the same classifier weights as above, i.e., $w_{\text {svm }}=3.73$ and $w_{\text {ann }}=3.10$. Now, the WMV vector $[\mathrm{A}, \mathrm{B}, \mathrm{C}, \mathrm{D}]$

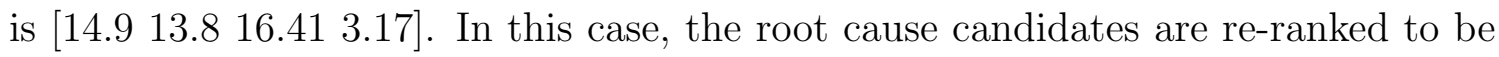
$\mathrm{C}, \mathrm{A}, \mathrm{B}, \mathrm{D}$. The candidate root cause $\mathrm{C}$ is raised from the third attempt to the first attempt, and repair can therefore be successfully carried out with the first attempt.

\subsection{Experiments and Results}

Experiments were performed on three industrial boards that are currently in highvolume production. Relevant information about two boards is provided in Table 2.1, and reproduced in Table 3.1. For the third board, a total of 1023 repaired boards are analyzed as training data. A total of 751 fault syndromes are extracted from failure logs. The number of faulty components for repair action is 109. Information about three boards are provided in Table 3.1

ANNs are implemented using the Neural Network Toolkit [57]. SVMs are imple- 
Table 3.1: Information about the industrial boards used for classification and the log data available.

\begin{tabular}{|c|c|c|c|}
\hline & Board 1 & Board 2 & Board 3 \\
\hline Number of syndromes & 546 & 375 & 751 \\
\hline $\begin{array}{c}\text { Number of repair } \\
\text { candidates (components) }\end{array}$ & 153 & 116 & 109 \\
\hline Number of boards & 1613 & 1351 & 1023 \\
\hline
\end{tabular}

mented using an open source SVM toolbox [51]. Diagnosis results were obtained for different designs of the ANNs, e.g., for various training functions, transfer functions, and number of neuron layers. The proposed one-layer ANNs with linear transfer function and LM-optimization training function has higher diagnosis accuracy and resolution. Diagnosis results for SVMs also vary with different parameters, e.g., kernel functions, kernel degrees, and penalty parameters. SVMs of linear kernel outperform (in terms of diagnosis accuracy) over the ANNs and the SVMs of other kernels. Furthermore, the diagnosis system based on weighted-majority voting provides even higher classification rates, thereby achieving better performance than the single machines. Experiments were run on a 64-bit Linux system with 12 GB of RAM and quad-core Intel i7 processors running at $2.67 \mathrm{GHz}$.

In this work, we use the same cross-validation methods and concepts of success ratios, as described in Chapter 2, to evaluate the performance of ANN-based, SVMbased, and WMV-based diagnosis systems.

\section{Diagnostic Results Using The ANNs-Based Learning System}

The diagnostic accuracy of the ANNs-based method is related to the transfer function and training algorithm. In Tables 3.2, we present the results of two training functions (trainrp and trainlm), and two transfer functions (linear and sigmoid). Function trainrp updates weight and bias values according to the resilient backpropagation algorithm. Function trainlm updates weight and bias values according 
Table 3.2: Diagnosis results for the ANN-based system.

(a) Board 1

\begin{tabular}{|c|c|c|c|c|}
\hline Transfer function & \multicolumn{2}{|c|}{ logsig } & \multicolumn{2}{c|}{ linear } \\
\hline Training function & trainrp & trainlm & trainrp & trainlm \\
\hline $\mathrm{SR}_{1}$ & $55.0 \%$ & $34.3 \%$ & $67.9 \%$ & $71.0 \%$ \\
\hline $\mathrm{SR}_{2}$ & $60.0 \%$ & $42.0 \%$ & $78.1 \%$ & $84.0 \%$ \\
\hline $\mathrm{SR}_{3} \mathrm{~s}$ & $64.0 \%$ & $44.0 \%$ & $84.4 \%$ & $90.6 \%$ \\
\hline Training time & $52.8 \mathrm{~s}$ & $83.1 \mathrm{~s}$ & $71.2 \mathrm{~s}$ & $52.2 \mathrm{~s}$ \\
\hline
\end{tabular}

(b) Board 2

\begin{tabular}{|c|c|c|c|c|}
\hline Transfer function & \multicolumn{2}{|c|}{ logsig } & \multicolumn{2}{c|}{ linear } \\
\hline Training function & trainrp & trainlm & trainrp & trainlm \\
\hline $\mathrm{SR}_{1}$ & $54.9 \%$ & $33.4 \%$ & $57.9 \%$ & $54.9 \%$ \\
\hline $\mathrm{SR}_{2}$ & $66.3 \%$ & $33.4 \%$ & $70.3 \%$ & $70.3 \%$ \\
\hline $\mathrm{SR}_{3}$ & $71.2 \%$ & $39.6 \%$ & $75.1 \%$ & $74.5 \%$ \\
\hline Training time & $42.4 \mathrm{~s}$ & $72.3 \mathrm{~s}$ & $60.2 \mathrm{~s}$ & $33.1 \mathrm{~s}$ \\
\hline
\end{tabular}

(c) Board 3

\begin{tabular}{|c|c|c|c|c|}
\hline Transfer function & \multicolumn{2}{|c|}{ logsig } & \multicolumn{2}{c|}{ linear } \\
\hline Training function & trainrp & trainlm & trainrp & trainlm \\
\hline $\mathrm{SR}_{1}$ & $68.7 \%$ & $58.0 \%$ & $71.3 \%$ & $69.4 \%$ \\
\hline $\mathrm{SR}_{2}$ & $71.3 \%$ & $58.3 \%$ & $75.2 \%$ & $74.6 \%$ \\
\hline $\mathrm{SR}_{3}$ & $72.6 \%$ & $58.6 \%$ & $76.5 \%$ & $76.2 \%$ \\
\hline Training time & $142.6 \mathrm{~s}$ & $420.4 \mathrm{~s}$ & $217.4 \mathrm{~s}$ & $96.2 \mathrm{~s}$ \\
\hline
\end{tabular}

to LM optimization.

From the Tables 3.2, we observe that the best combination of transfer function and training function in terms of diagnostic accuracy and training time for board diagnosis is trainlm and linear, and the corresponding results are the last column of each table. It is fast and often the first-choice ANN algorithm in the Matlab toolbox [57]. For example in Board 1, the $\mathrm{SR}_{1}$ for $\mathrm{ANN}$ of trainlm and linear is $71.0 \%$, while the SR is $67.9 \%$ for ANN of trainrp and linear, $55.0 \%$ for ANN of trainrp and logsig, and $34.3 \%$ for ANN of trainrp and linear. The training time for ANN of trainlm and linear is also lower than the other three combinations. For Board 
Table 3.3: Comparison of SRs obtained by ANNs and BI.

(a) Board 1

\begin{tabular}{|c|c|c|c|c|}
\hline & $\mathrm{SR}_{1}$ & $\mathrm{SR}_{2}$ & $\mathrm{SR}_{3}$ & Training time \\
\hline Proposed ANNs & $67.9 \%$ & $78.1 \%$ & $84.4 \%$ & $71.3 \mathrm{~s}$ \\
\hline One-layer ANNs (no weight initialization) & $66.7 \%$ & $78.1 \%$ & $82.0 \%$ & $72.8 \mathrm{~s}$ \\
\hline Traditional ANNs (20 hidden neurons) & $60.3 \%$ & $70.7 \%$ & $75.3 \%$ & $4 \mathrm{~h}$ \\
\hline Bayesian inference & $62.5 \%$ & $72.5 \%$ & $76.2 \%$ & $21.2 \mathrm{~s}$ \\
\hline
\end{tabular}

(b) Board 2

\begin{tabular}{|c|c|c|c|c|}
\hline & $\mathrm{SR}_{1}$ & $\mathrm{SR}_{2}$ & $\mathrm{SR}_{3}$ & Training time \\
\hline Proposed ANNs & $57.9 \%$ & $70.3 \%$ & $75.1 \%$ & $60.2 \mathrm{~s}$ \\
\hline One-layer ANNs (no weight initialization) & $53.3 \%$ & $61.6 \%$ & $73.0 \%$ & $51.6 \mathrm{~s}$ \\
\hline Traditional ANNs (20 hidden neurons) & $46.6 \%$ & $63.3 \%$ & $79.6 \%$ & $1 \mathrm{~h}$ \\
\hline Bayesian inference & $42.1 \%$ & $55.6 \%$ & $62.4 \%$ & $15.2 \mathrm{~s}$ \\
\hline
\end{tabular}

(c) Board 3

\begin{tabular}{|c|c|c|c|c|}
\hline & $\mathrm{SR}_{1}$ & $\mathrm{SR}_{2}$ & $\mathrm{SR}_{3}$ & Training time \\
\hline Proposed ANNs & $69.4 \%$ & $74.6 \%$ & $76.2 \%$ & $46.2 \mathrm{~s}$ \\
\hline One-layer ANNs (no weight initialization) & $53.2 \%$ & $66.5 \%$ & $70.8 \%$ & $47.9 \mathrm{~s}$ \\
\hline Traditional ANNs (20 hidden neurons) & $48.9 \%$ & $61.4 \%$ & $64.2 \%$ & $6 \mathrm{~h}$ \\
\hline Bayesian inference & $43.7 \%$ & $47.3 \%$ & $59.3 \%$ & $31.4 \mathrm{~s}$ \\
\hline
\end{tabular}

1 in Table 3.2.(a), the training time for ANN of trainlm and linear is 52.2 seconds, which is lower than the other three where the training times are 52.8 seconds, 83.1 seconds and 71.2 seconds, respectively.

We also found that the diagnosis accuracy is related to the number of training cases. Board 1 and Board 3 have more training cases than Board 2. The classification models of these two boards can be trained more accurately, thus the diagnosis accuracies of these two boards are higher. We observe that the training time is correlated to the number of classes, since the number of constructed ANN networks is as the same as the number of classes, as described in Section 3.2. For Board 3, 109 neural networks are constructed for 109 root fault classes, which is much more than that for Board 1 and Board 2. 
We compare the diagnosis success ratio obtained using the proposed ANNs to that obtained using traditional ANNs and Bayesian inference (BI) [49], with the same training cases and test cases. For traditional ANNs, we consider a 3-layer network [60]. The number of neurons in hidden layer is 20. We adjust the number of neurons in the hidden layer to observe the variation of the $\mathrm{SR}_{1}$. A total of six different numbers are used, respectively, 5, 10, 20, 50, 100, 500. For example, for Board 1 in Table 3.3.(a), when the number of neurons in the hidden layer is equal to 20 , we obtain the highest $\mathrm{SR}_{1}$ to be $(60.3 \%)$. When the number is equal to 500 , we obtain the the lowest $\mathrm{SR}_{1}$ to be (11.4\%). In the comparison shown in Tables 3.3, we list the highest $\mathrm{SR}_{1}$ achieved by traditional ANNs. However, it is still much lower than the success ratio achieved by the proposed ANNs. The difference of $16 \%$ in the $\mathrm{SR}_{1}$ and the difference of $10 \%$ in the success within three attempts is especially significant for high-volume production. The training algorithm used for the architectures is trainrp. In the Bayesian-based method, first, we calculate the prior probability based on the training cases, which is the occurrence probability of a syndrome given a repair action. For example, suppose that a particular repair action has been taken 100 times, and a given syndrome showed up 80 times in these 100 cases. Then the prior probability is 0.8 . All other occurrence probabilities of syndromes, given repair actions, can be calculated in the same manner. After obtaining the prior probabilities, we can calculate the posterior probability based on Bayes' formula. We take all the fault syndromes into account in the calculation of the posterior probability. For each action, the times of calculation of the posterior probability is equal to the number of fault syndromes. Therefore, the final probability of an action being correct is obtained using the information reflected by all the fault syndromes. More details are described in [49].

The success ratios within 3 attempts are listed in Tables 3.3. We can see that the proposed ANNs based method has an obvious advantage over the Bayesian inference 
Table 3.4: Comparison of SRs obtained by SVMs with different kernels.

(a) Board 1

\begin{tabular}{|c|c|c|c|c|c|}
\hline & Linear & \multicolumn{3}{|c|}{ Polynomial kernel } & Gaussian \\
\cline { 3 - 5 } & kernel & $d=2$ & $d=3$ & $d=4$ & kernel \\
\hline $\mathrm{SR}_{1}$ & $73.2 \%$ & $74.4 \%$ & $72.3 \%$ & $74.9 \%$ & $62.6 \%$ \\
\hline $\mathrm{SR}_{2}$ & $80.4 \%$ & $82.2 \%$ & $81.2 \%$ & $82.7 \%$ & $74.0 \%$ \\
\hline $\mathrm{SR}_{3}$ & $88.2 \%$ & $91.9 \%$ & $90.4 \%$ & $91.9 \%$ & $79.2 \%$ \\
\hline Training time (sec) & 43.2 & 45.2 & 41.1 & 42.0 & 49.9 \\
\hline
\end{tabular}

(b) Board 2

\begin{tabular}{|c|c|c|c|c|c|}
\hline & Linear & \multicolumn{3}{|c|}{ Polynomial kernel } & Gaussian \\
\cline { 3 - 5 } & kernel & $d=2$ & $d=3$ & $d=4$ & kernel \\
\hline $\mathrm{SR}_{1}$ & $66.3 \%$ & $63.2 \%$ & $63.2 \%$ & $66.3 \%$ & $66.3 \%$ \\
\hline $\mathrm{SR}_{2}$ & $74.3 \%$ & $70.1 \%$ & $70.1 \%$ & $75.5 \%$ & $74.3 \%$ \\
\hline $\mathrm{SR}_{3}$ & $84.1 \%$ & $79.5 \%$ & $78.5 \%$ & $82.1 \%$ & $83.5 \%$ \\
\hline Training time (sec) & 23.6 & 25.9 & 21.6 & 22.5 & 29.1 \\
\hline
\end{tabular}

(c) Board 3

\begin{tabular}{|c|c|c|c|c|c|}
\hline & Linear & \multicolumn{3}{|c|}{ Polynomial kernel } & Gaussian \\
\cline { 3 - 5 } & kernel & $d=2$ & $d=3$ & $d=4$ & kernel \\
\hline $\mathrm{SR}_{1}$ & $73.5 \%$ & $70.0 \%$ & $69.0 \%$ & $69.0 \%$ & $71.7 \%$ \\
\hline $\mathrm{SR}_{2}$ & $82.7 \%$ & $80.3 \%$ & $80.3 \%$ & $80.3 \%$ & $82.1 \%$ \\
\hline $\mathrm{SR}_{3}$ & $84.0 \%$ & $82.6 \%$ & $83.6 \%$ & $83.6 \%$ & $82.1 \%$ \\
\hline Training time (sec) & 80.4 & 91.4 & 72.3 & 75.3 & 82.2 \\
\hline
\end{tabular}

in terms of success ratio. For example in Board 1, the $\mathrm{SR}_{1}$ of the proposed ANNs, ANNs without initial weights, traditional ANNs, and Bayesian inference are $67.9 \%$, $66.7 \%, 60.3 \%$, and $62.5 \%$, respectively. For Board 1, the training time of the proposed ANNs, ANNs without initial weights, traditional ANNs, and Bayesian inference are $71.3 \mathrm{sec}, 72.8 \mathrm{sec}, 4$ hours, and $21.2 \mathrm{sec}$, respectively. The training time of a 20-layer ANN for Board 3 is over 6 hours while the training times are less than 1 minute for the other three methods. A 20-layer ANN has many more hidden layers and hidden neurons than the proposed 1-layer ANN. To calculate the weights and offset of hidden layers, we require an exponential amount of CPU time. Therefore, the 
calculation time for the 20-layer ANNs is exponentially larger than simple 1-layer ANNs.

\section{Diagnostic Results Using The SVMs-Based Learning System}

We apply different types of kernel functions to the SVM-based learning system. The linear kernel, the polynomial kernel (degree 2, 3, and 4), and the Gaussian kernel, are commonly used in SVM training and are therefore implemented in our experiments. The diagnosis results for the four boards are listed in Tables 3.4. We observe that the linear kernel function provides the highest SR on our test cases. For example, for Board 1 in Table 3.4.(a), the $\mathrm{SR}_{1}$ for SVMs with the linear kernel is up to $73.2 \%$ and the $\mathrm{SR}_{3}$ is up to $88.2 \%$. In addition, the training times of linear-kernel SVMs and polynomial-kernel SVMs are less than the training time of ANNs in Tables 3.23.3. The training time of SVMs in this section is referred to the time consumed in selecting support vectors and calculating weights of the support vectors for all the SVMs using the training set.

Another interesting parameter in the SVM algorithm is the penalty $\mathrm{C}$; a large value of $\mathrm{C}$ corresponding to the assignment of a higher penalty to misclassification. In this sense, a higher value of C leads to more effective SVM training in terms of less training errors, therefore improving model generality, which in turn results in a higher diagnosis accuracy. However, a larger penalty $\mathrm{C}$ also leads to the overfitting of the SVM model. Detailed results on diagnosis accuracy obtained by varying parameter $\mathrm{C}$ are presented in [3]. In [3], the penalty parameters are varied from infinity to 0.001 . When $\mathrm{C}$ is less than 0.1 , the $\mathrm{SR}_{1}$ drop significantly. Therefore, the penalty parameter has to be relatively large to ensure that SVMs can adequately represent the training set, and thus providing accurate diagnosis. Here, we use $\mathrm{C}=1000$ throughout as in [3] for SVM training. Besides the penalty parameter C, Gaussian kernel-based SVMs require an additional parameter, namely the kernel width $\sigma^{2}$. A very small or 
very large $\sigma^{2}$ leads to either over-fitting or under-fitting [63]. Nevertheless, we provide in Tables 3.4 the highest SRs obtained using SVMs with Gaussian kernels and best value of $\sigma^{2}$. The implementation of Gaussian kernel-based SVMs is impractical since $\sigma^{2}$ must be individually determined for different boards using exhaustive search.

Diagnostic Results Using Weighted-Majority Voting

We next present results for WMV, where a combination of ANN and SVM is used to achieve higher diagnosis accuracy. The weights of two classifiers, $w_{\text {svm }}$ and $w_{\text {ann }}$, are based on the training errors, which are generated dynamically while the models are built. Linear-kernel SVMs and one-layer ANNs are used as the two learning methods in the combined WMV diagnosis system. We observe that the training errors of SVMs for most boards are less than that of ANNs, with Board 2 being the only exception. The corresponding classifier weights are shown in the captions of Tables 3.5. Therefore, in most cases, the decisions provided by the SVM diagnosis subsystem has a greater influence on WMV diagnosis, while ANNs serve as an secondary decision engine to verify or contradict the diagnosis decisions obtained from SVMs.

In our experiment, we investigate the contributions to the SR for three attempts from the two constituent diagnosis systems. Intuitively, we should assign the highest weight to the first attempt, next highest weight to the second attempt, and lowest to the third attempt. However, we cannot arbitrarily judge the optimal configuration for the weight combination. We conducted experiments to assess different weight configurations. Since we select three optimal attempts from a total of three to six potential repair attempts provided by two diagnosis systems, the third attempt in single diagnosis system is least likely to be the correct choice compared to the first two attempts. Thus we assign a small weight of 1 to the third attempt. The second attempt is then assigned a larger weight of 2 because it is more accurate than the third attempt. We consider the weights of the first attempt to be 3, 4, and 5. Therefore, 
Table 3.5: Comparison of SRs obtained by ANNs, SVMs, and WMV.

(a) Board $1 w_{\text {svm }}=6.63$ and $\left.w_{\text {ann }}=5.19\right)$

\begin{tabular}{|c|c|c|c|}
\hline & $\mathrm{SR}_{1}$ & $\mathrm{SR}_{2}$ & $\mathrm{SR}_{3}$ \\
\hline Proposed ANNs & $67.9 \%$ & $78.1 \%$ & $84.4 \%$ \\
\hline Proposed SVMs & $73.2 \%$ & $80.4 \%$ & $88.2 \%$ \\
\hline WMV (3-2-1) & $78.5 \%$ & $80.4 \%$ & $89.5 \%$ \\
\hline WMV (4-2-1) & $78.5 \%$ & $82.5 \%$ & $89.5 \%$ \\
\hline WMV (5-2-1) & $78.5 \%$ & $82.5 \%$ & $89.5 \%$ \\
\hline
\end{tabular}

(b) Board $2 w_{\text {svm }}=3.63$ and $w_{\text {ann }}=2.19$ )

\begin{tabular}{|c|c|c|c|}
\hline & $\mathrm{SR}_{1}$ & $\mathrm{SR}_{2}$ & $\mathrm{SR}_{3}$ \\
\hline Proposed ANNs & $57.9 \%$ & $70.3 \%$ & $75.1 \%$ \\
\hline Proposed SVMs & $66.3 \%$ & $74.3 \%$ & $84.1 \%$ \\
\hline WMV (3-2-1) & $69.0 \%$ & $75.0 \%$ & $87.0 \%$ \\
\hline WMV (4-2-1) & $69.0 \%$ & $76.7 \%$ & $87.0 \%$ \\
\hline WMV (5-2-1) & $69.0 \%$ & $76.7 \%$ & $89.4 \%$ \\
\hline
\end{tabular}

(c) Board $3\left(w_{\text {svm }}=6.63\right.$ and $\left.w_{\text {ann }}=5.19\right)$

\begin{tabular}{|c|c|c|c|}
\hline & $\mathrm{SR}_{1}$ & $\mathrm{SR}_{2}$ & $\mathrm{SR}_{3}$ \\
\hline Proposed ANNs & $69.4 \%$ & $74.6 \%$ & $76.3 \%$ \\
\hline Proposed SVMs & $73.5 \%$ & $82.7 \%$ & $84.0 \%$ \\
\hline WMV (3-2-1) & $73.0 \%$ & $83.4 \%$ & $86.8 \%$ \\
\hline WMV (4-2-1) & $75.6 \%$ & $83.4 \%$ & $86.8 \%$ \\
\hline WMV (5-2-1) & $75.6 \%$ & $83.4 \%$ & $86.8 \%$ \\
\hline
\end{tabular}

the weighting strategies are 3-2-1, 4-2-1, and 5-2-1. The choice of weight is empirical, but these weights define the relative importance of different repair attempts.

The diagnosis results are shown in Tables 3.5. We observe that the diagnosis accuracy for WMV is at least as good as the best single diagnosis system. In particular, WMV performs better for boards with low volume and where the diagnosis accuracy is low for a single diagnosis system. We observe the improvements on Board 1 in Table 3.5.(a). The $\mathrm{SR}_{1}$ is raised from $67.9 \%$ using $\mathrm{ANNs}$ and $73.2 \%$ using SVMs to 78.5\% using WMV. The SR for third attempt is raised from $84.4 \%$ using ANNs and $88.2 \%$ in SVMs to $89.5 \%$ using WMV. We also observe that the choices of different 


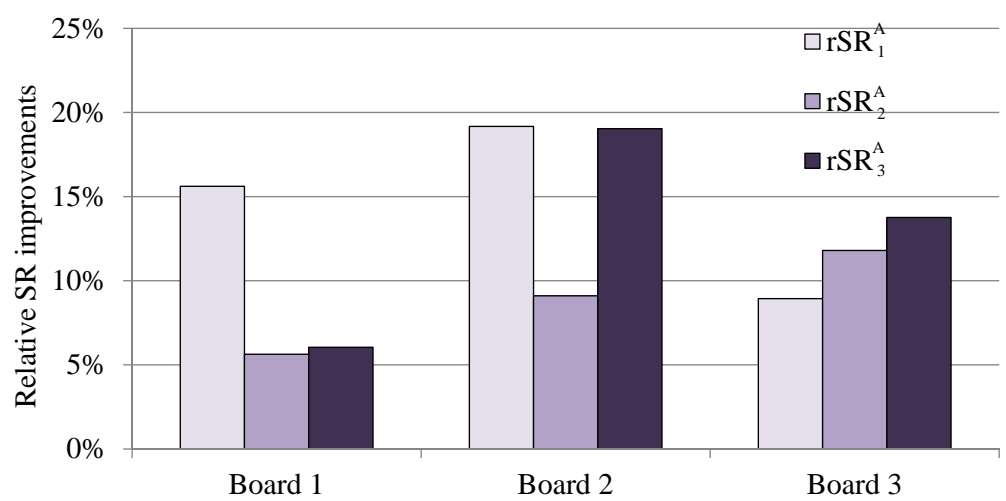

FigURE 3.5: SR improvement when WMV is used instead of ANNs.

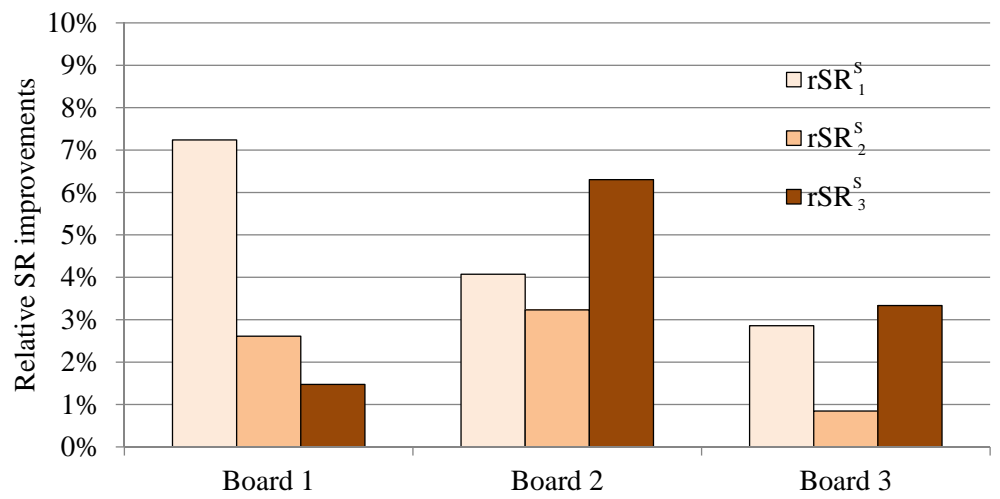

FiguRE 3.6: SR improvement when WMV is used instead of SVMs.

weights do not show substantial difference in diagnosis accuracy; However, we recommend the weighting strategy of 4-2-1 since this set of weights always provide the highest diagnosis accuracy in this chapter among the three weighting strategies.

We illustrate the improvement in diagnosis accuracy by comparing relative SR percentage increase for the three boards; see Figure 3.5 and Figure 3.6. The WMV method uses the weights of 4-2-1. We observe that the SR improvements for boards with fewer training cases are more than those with more training cases. Let $\mathrm{SR}_{i}^{A}$ $\left(\mathrm{SR}_{i}^{S}\right)$ be the SR with $i$ attempts for the baseline method ANNs (SVMs), Let $\mathrm{SR}_{i}^{W}$ be the SR achieved with $i$ attempts using WMV. The relative percentage increase in SRs is defined as $\mathrm{rSR}_{i}^{A}=\left(\left(\mathrm{SR}_{i}^{W}-\mathrm{SR}_{i}^{A}\right) / \mathrm{SR}_{i}^{A}\right) \times 100 \%$ in Figure 3.5 and $\mathrm{rSR}_{i}^{S}=$ 
$\left(\left(\mathrm{SR}_{i}^{W}-\mathrm{SR}_{i}^{S}\right) / \mathrm{SR}_{i}^{S}\right) \times 100 \%$ in Figure 3.6. For Board 1, $\mathrm{SR}_{1}, \mathrm{SR}_{2}$, and $\mathrm{SR}_{3}$ increase from $67.9 \%, 78.1 \%$, and $84.4 \%$ for the ANN baseline and $73.2 \%, 80.4 \%$, and $88.2 \%$ for the SVM baseline to $78.5 \%, 82.5 \%$, and $89.5 \%$ using WMV. Therefore, the values of $\mathrm{rSR}_{1}^{A}, \mathrm{rSR}_{2}^{A}$, and $\mathrm{rSR}_{3}^{A}$ for Board 1 are $15.9 \%, 5.3 \%$, and 7.1\%, respectively in Figure 3.5 and the values of $\mathrm{rSR}_{1}^{S}, \mathrm{rSR}_{2}^{S}$, and $\mathrm{rSR}_{3}^{S}$ are $7.3 \%, 2.6 \%$, and $1.7 \%$, respectively in Figure 3.6.

\section{Summary of Contributions}

The proposed method is a significant improvement over the traditional manual diagnosis and diagnostic software currently used in production. With three attempts, the diagnostic success ratio of the diagnostic software currently used in the production line is less than $50 \%^{1}$ and even poorer when the volume of boards are small. The first attempt SR is considerably lower. Using the proposed diagnostic system, more faulty boards can be successfully repaired within three attempts. To better understand what these results imply, consider the following hypothetical (yet representative) example. Suppose 5000 boards are fabricated per month by a manufacturer; each board costs $\$ 3000$ and $10 \%$ of the boards fail functional test. If we can improve the repair success ratio by even a small percentage, for example $5 \%$, using the new diagnostic system. This means that the board manufacturer saves $\$ 75,000$ per month for every $5 \%$ improvements in success ratios.

\subsection{Conclusions}

This chapter has described a smart diagnosis system based on a combination of artificial neural networks and support vector machines to rapidly and correctly locate the root failure causes on modern circuit boards. The proposed diagnosis system was designed to automate syndrome extraction or preparation for diagnosis and system

1 Exact success ratios are not presented here in order to protect company-confidential data. 
training. The relationship between log files and potential fault components cannot be inferred from visual inspection. Artificial neural networks can generate a visual relationship between syndrome and root cause, and support vector machine creates an optimal hyper plane to separate the root cause in syndrome space. Weightedmajority voting was proposed to take advantage of both ANNs and SVMs to provide an optimal repair suggestion set. Three industrial boards, which are currently in high-volume production were used to validate the effectiveness of the proposed method. 
4

\section{Adaptive Diagnosis Using Decision Trees}

Functional fault diagnosis at board-level is desirable for high-volume production since it improves product yield. However, to ensure diagnosis accuracy and effective board repair, a large number of syndromes must be used. Therefore, the diagnosis cost can be prohibitively high due to the increase in diagnosis time and the complexity of syndrome collection/analysis.

In this chapter, we apply decision trees to the problem of adaptive board-level functional fault diagnosis in high-volume manufacturing. The number of syndromes used for diagnosis is significantly less than the number of syndromes used for $a$ priori training. Despite an order of magnitude or higher reduction in the number of syndromes compared to SVMs and ANNs, the diagnosis accuracy of the proposed method is comparable to that for the baseline methods and considerably higher for the same number of syndromes. Another advantage of using decision trees is that it can also be used to select an effective, but reduced, set of syndromes for use by ANNs or SVMs in a subsequent step. Moreover, to enable the reuse of knowledge from the test-design stage, we use an incremental version of decision trees so as to bridge the knowledge gap between test-design stage and volume production stage. 
The remainder of this chapter is organized as follows. Section 4.1 presents the problem and describes the work contributions. Section 4.2 describes the diagnosis system based on DTs. The architecture of DTs and advantages of this architecture are presented. Section 4.3 describes the use of incremental learning in DTs. Section 4.4 describes the diagnosis flow using incremental DTs. Section 4.5 presents the results for two industrial boards. The substantial reduction in the number of syndromes highlights the effectiveness of the proposed DT-based diagnostic system. Finally, Section 4.6 concludes the chapter.

\subsection{Problem Statement and Chapter Contributions}

The proposed diagnostic system is based on incremental decision trees (DTs). A trained DT model consists of a tree-like diagnosis flow, described in detail in Section 4.2. The discriminative ability of syndrome is analyzed based on statistical criterion, such as Gini Index or Information Gain $[64,65]$. The most discriminative syndrome is selected to be the starting point for diagnosis. The final repair suggestion is available at the leaf node of a DT. The diagnosis time can be significantly reduced using DTs, since only a small number of syndromes are needed for identifying the root cause, instead of all the syndromes that are used in ANNs and SVMs [3,4]. As shown in Section 4.5 for industry boards, only tens of syndromes on average are required for identifying the root cause. Furthermore, an advantage of DT is its interpretation of the relationship between syndromes and corresponding root causes.

Unlike the diagnosis procedures of ANNs and SVMs that use all the syndromes simultaneously for diagnosis, the diagnosis procedure of DTs relies on a multi-stage or sequential approach to the problem. In fact, it resembles the steps followed by an experienced debug technician. When a DT-based diagnosis system receives a faulty board, it starts by determining and analyzing the most discriminative syndrome. Based on results obtained using this syndrome, the diagnosis system can locate a 
group of potential root causes and also identify the second-most discriminative syndrome, and so on. After several syndromes are determined in this way and analyzed, the most-likely root cause candidate is suggested for repair. In practice, skilled debug engineers usually follow such a tree-like diagnosis procedure, which is derived from their experience. This knowledge can be recorded in the same form of a decision tree. Moreover, in a typical volume-production environment, diagnosis knowledge is collected in a dynamic manner as more faulty boards are detected after testing. Hence, the diagnosis system must also adapt to new knowledge corresponding to various error scenarios. By using incremental decision trees, we bridge the knowledge gap between what debug engineers know at test-design stage and the information gained through testing during volume production; see Fig. 4.1.

The merits of using the proposed adaptive functional fault diagnosis system are:

- Reduced and selective set of syndromes needed for diagnosis. Syndrome selection is based on the information derived from the previous syndrome.

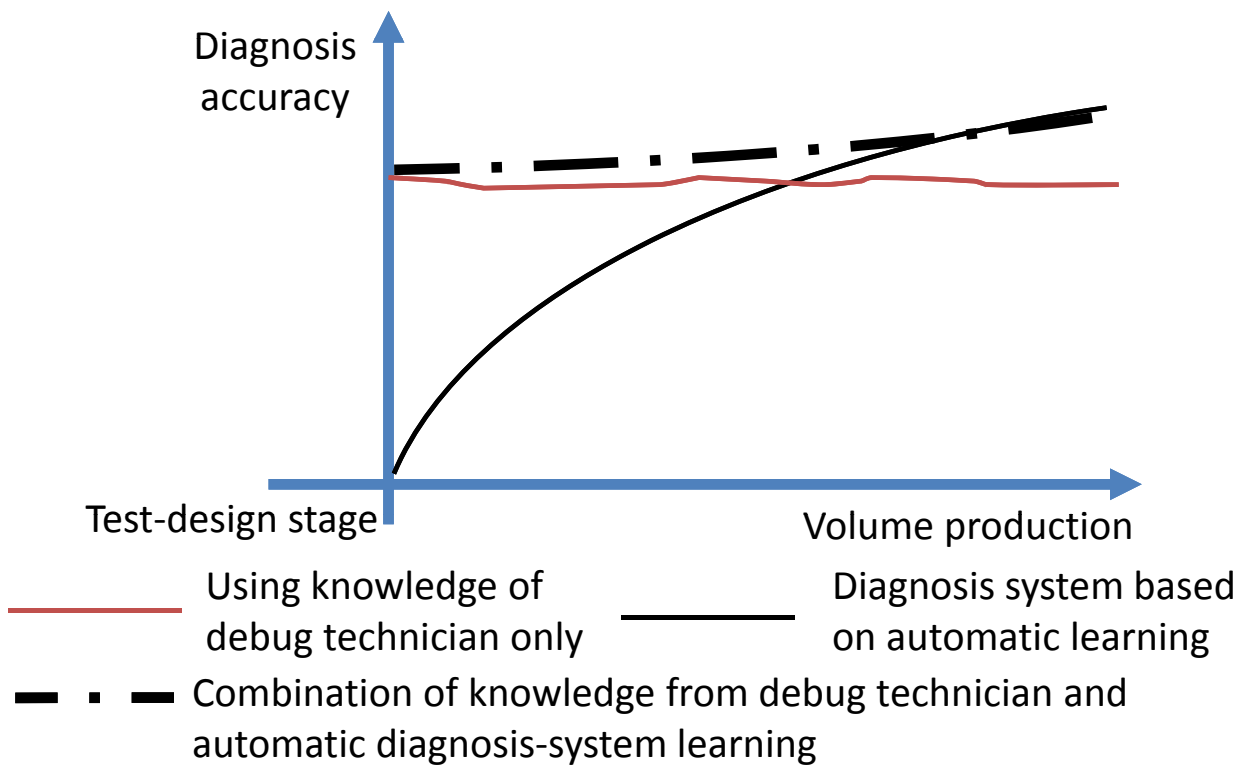

FigURE 4.1: Illustration of the benefit of bridging the knowledge gap between debug technicians and an automated learning system. 
- Automatic integration of knowledge of newly identified error scenarios during volume production and field deployment in the field to the DT-based diagnosis system.

- Utilization of knowledge from debug technicians at test-design stage along with the knowledge of failing boards gained continuously in the field.

\subsection{Decision Trees}

Decision trees (DTs) are widely used in statistics, data mining, and machine learning, as a predictive model for mapping observations of an item to its targeted value [64]. A final decision is reached based on a series of observations and the associated decision steps. In the early days of decision theory, the DT model was constructed manually. DT learning was subsequently introduced in [65], as a result of which the DT model could be constructed automatically. DTs are now applied to various fields as a powerful learning and classification tool, e.g., market prediction, disease prediction [66], etc.

A DT consists of two types of nodes, leaf(terminal) nodes and decision(internal) nodes. Leaf nodes refer to the nodes that do not branch and contain class information. Decision nodes refer to the nodes that can branch to multiple child nodes or leaf nodes. Based on the inference made from the current decision node, a child node is selected for further branching. Suppose we have a faulty board with several root-cause candidates denoted by the elements of the set $\boldsymbol{A}=\left\{A_{1}, A_{2} \ldots A_{m}\right\}$. These root-cause candidates are the leaf nodes in the decision tree. To diagnose the root cause for this faulty board, we have a number of syndromes for diagnosis, i.e., $\boldsymbol{T}=$ $\left\{T_{1}, T_{2} \ldots T_{n}\right\}$, which are encoded in the decision nodes. Each decision node $T_{i}$ has two branches, i.e., $t_{i}$ and $\overline{t_{i}}$, where $t_{i}$ denotes that the syndrome $T_{i}$ manifests itself, and $\overline{t_{i}}$ otherwise. Figure 4.2 illustrates the structure of a decision tree for board diag- 


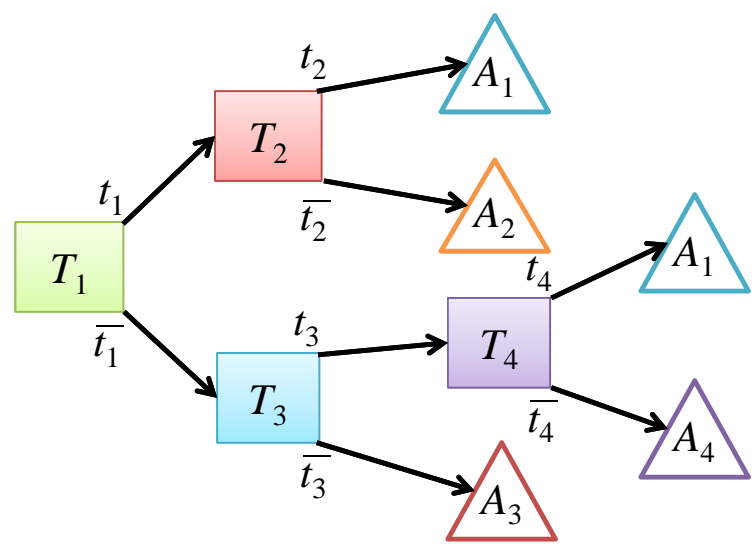

FIGURE 4.2: Illustration of a decision tree.

nosis. For example, two sequences of syndrome observations lead to the prediction of root cause $A_{1}$ in Figure 4.2: 1) $\left.t_{1} \rightarrow t_{2} \rightarrow A_{1} ; 2\right) \overline{t_{1}} \rightarrow t_{3} \rightarrow t_{4} \rightarrow A_{1}$. Both diagnosis procedures require less syndromes than the total number of syndromes in the tree. A reduction in the number of tests results in significant reduction in diagnosis time.

\subsubsection{Training of Decision Trees}

During the decision-making process, different observations on a current internal node lead to different child nodes for further classification. Therefore, determining the discriminative ability of the feature is critical in decision-tree training. DT training involves the recursive partitioning of the training data, which is split into increasingly homogeneous subsets on the basis of a splitting criterion. There are several common criteria to choose from, such as Information Gain [65], Gini Index [64], and Twoing [64]. These are described below.

\section{Information Gain}

Let $\boldsymbol{C}$ denote a set of training cases (faulty boards), which can be labeled by a set of classes (root causes) $\boldsymbol{A}=\left\{A_{1}, A_{2} \ldots A_{m}\right\}$. The test set $\boldsymbol{T}=\left\{T_{1}, T_{2} \ldots T_{n}\right\}$ can be deemed to be a vector of features attached to the classes, where $n$ is the dimension 
of the feature space. Our goal is to select the most discriminative feature $T_{i}$ from $\boldsymbol{T}$. The expected information obtained for each feature $T_{i}$ is measured by its gain $I G\left(\boldsymbol{C}, T_{i}\right)$, defined below:

$$
I G\left(\boldsymbol{C}, T_{i}\right)=E(\boldsymbol{C})-E\left(\boldsymbol{C} \mid T_{i}\right)
$$

where $E(\boldsymbol{C})$ is the entropy of $\boldsymbol{C}$, i.e., $E(\boldsymbol{C})=-\sum_{j} p\left(A_{j}\right) \log p\left(A_{j}\right)$, with $p\left(A_{j}\right)$ being the probability of case with root cause $A_{j} \in \boldsymbol{A}$ being in the training set $\boldsymbol{C}$. In the same way, $E\left(\boldsymbol{C} \mid T_{i}\right)=-\sum_{j} p\left(A_{j} \mid t_{i}\right) \log p\left(A_{j} \mid \overline{t_{i}}\right)$, where $t_{i}$ is the observation of $T_{i}$ manifests itself, and $\overline{t_{i}}$ otherwise.

A rule of thumb is to branch on the feature that provides the most information gain [65]. information gain.

\section{Gini Index}

The Gini Index criterion is based on Gini impurity $G I\left(\boldsymbol{C}, T_{i}\right)$, given as follow:

$$
G I\left(\boldsymbol{C}, T_{i}\right)=\operatorname{Gini}\left(\boldsymbol{C} \mid T_{i}\right)-\operatorname{Gini}(\boldsymbol{C})
$$

where $\operatorname{Gini}(\boldsymbol{C})$ is the Gini Index of $\boldsymbol{C}: \operatorname{Gini}(\boldsymbol{C})=\sum_{j} p\left(A_{j}\right)\left(1-p\left(A_{j}\right)\right)$. In the same way, $\operatorname{Gini}\left(\boldsymbol{C} \mid T_{i}\right)=\sum_{j} p\left(A_{j} \mid t_{i}\right)\left(1-p\left(A_{j} \mid \bar{t}_{i}\right)\right)$.

The rule here is to branch on the feature that has the lowest Gini impurity [65].

\section{Twoing}

The Twoing criterion splits classes by grouping the two most discriminative superclasses, rather than identifying each class from the remaining classes as in the Gini Index criterion. This criterion is useful for multiclass classifications. The Twoing value is defined as follows:

$$
\operatorname{Two}\left(T_{i}\right)=\left(\sum_{k \in m}\left(\frac{L_{k}}{\left(T_{i}\right)_{L}}-\frac{R_{k}}{\left(T_{i}\right)_{R}}\right)\right)^{2}
$$


where $\left(T_{i}\right)_{L} /\left(T_{i}\right)_{R}$ is the number of cases on the left/right of the decision node $T_{i}$,

and $L_{k} / R_{k}$ is the number of cases with the super root-cause $A_{k}$ on the left/right of the decision node $T_{i}$. The rule here is to branch on the feature that has the lowest Twoing value [65].

Based on the above discussion, we note that DT training generates a tree-like model, where the root node is the most discriminative feature and leaf nodes correspond to the labels of classes. In the prediction stage, the classifier examines the syndromes one-by-one by traversing the branches in the decision tree. In each step, the choice of next syndrome is determined by the observation of the current syndrome. Unlike the diagnosis procedure of ANNs and SVMs that analyze observations of all the syndromes based on a trained classifier, DTs require only a small number of syndromes for diagnosis.

\subsubsection{Example of DT-based Training and Diagnosis}

We next present an example of training a DT-based diagnosis system based on a board-repair database. Consider a hypothetical demonstration board with eight cases that are used for training. We build a DT model to identify faults for new cases. All the test-outcome information is stored in a log file, a sample of which is shown in Figure 1.8. The extracted syndromes and replaced components are used as inputs and outputs for the training of the DTs. Let $T_{1}, T_{2}, T_{3}$, and $T_{4}$ be four syndromes. If the syndrome $T_{i}$ manifests itself, we record it as $t_{i}$, and $\overline{t_{i}}$ otherwise. In a real scenario, fault syndromes vary across products and tests. Let us suppose that the board has four candidate root-cause components $A_{1}, A_{2}, A_{3}$, and $A_{4}$, respectively. Here, we merge the syndromes and the known root causes in one matrix $\mathcal{C}=[\mathcal{T} \mid \mathcal{A}]$, where the left side $(\mathcal{T})$ refers to the outcomes of tests, while the right side $(\mathcal{A})$ refers to the corresponding fault classes. This matrix represents the training information 
for the DTs.

$$
\mathcal{C}=[\mathcal{T} \mid \mathcal{A}]=\left[\begin{array}{cccc:c}
\overline{t_{1}} & \overline{t_{2}} & t_{3} & t_{4} & A_{1} \\
\overline{t_{1}} & \overline{t_{2}} & \overline{t_{3}} & t_{4} & A_{1} \\
\overline{t_{1}} & \overline{t_{2}} & t_{3} & t_{4} & A_{1} \\
\overline{t_{1}} & \overline{t_{2}} & \overline{t_{3}} & \overline{t_{4}} & A_{2} \\
\overline{t_{1}} & t_{2} & \overline{t_{3}} & \overline{t_{4}} & A_{2} \\
t_{1} & t_{2} & \overline{t_{3}} & \overline{t_{4}} & A_{3} \\
t_{1} & \overline{t_{2}} & \overline{t_{3}} & \overline{t_{4}} & A_{3} \\
t_{1} & \overline{t_{2}} & t_{3} & t_{4} & A_{4} \\
t_{1} & t_{2} & t_{3} & t_{4} & A_{4}
\end{array}\right]
$$

We consider the splitting criterion based on information gain [65]. We calculate the information gains for all the syndromes and classes. According to Equation (4.1), information gains for the individual syndromes are calculated to be 1.97 for $T_{1}, 1.31$ for $T_{2}, 1.67$ for $T_{3}$, and 1.97 for $T_{4}$. Therefore, we select the syndrome $T_{1}$ with the highest information gain as the root node for our diagnosis system; note that $T_{4}$ could also have been selected because it provides the same information gain. These nine cases can be divided into two groups, i.e., the first five (collectively referred to as $\mathcal{C}_{1}$ ) and the last four (collectively referred to as $\mathcal{C}_{2}$ ). We continue to split these two sets of data. For $\mathcal{C}_{1}$, we can choose $T_{4}$ with the highest $I G=0.97$, and $T_{4}$ is chosen to be the splitting node again for $\mathcal{C}_{2}$. Since splitting stops when all instances have the same root causes in the subgroup after splitting, the DT in this example consists of three decision nodes and four leaf nodes. Figure 4.3 shows the trained DT. The decision tree consists of one decision node of $T_{1}$ and two decision nodes arising from $T_{4}$. For example, the observation sequence $t_{1} \rightarrow \overline{t_{4}}$ gives rise to the prediction of the root cause $A_{1}$. In this example, the total number of syndromes used for diagnosis is reduced from 4 to 2 .

Next, suppose a new failing board is received and it has the syndrome $\left[\overline{t_{1}} \overline{t_{2}} t_{3} t_{4}\right]$, which corresponds to the first row (case) of $\mathcal{C}$ in Equation (4.4). This faulty board 


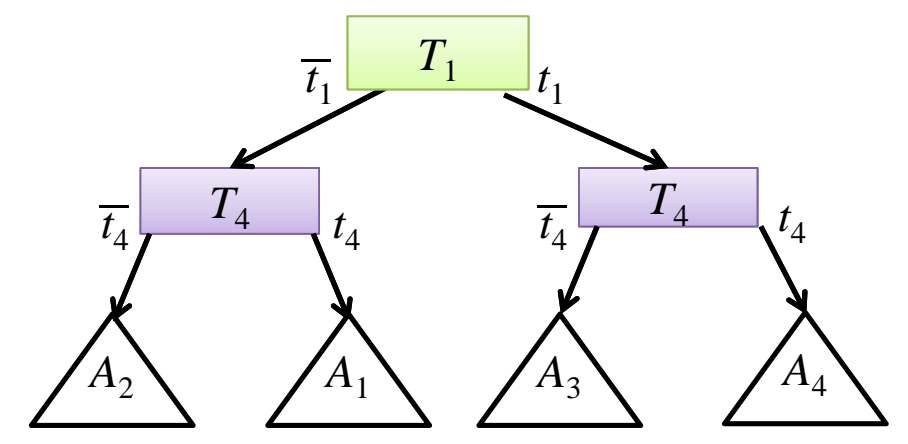

FiguRE 4.3: DT trained for demo board.

is first evaluated using syndrome $T_{1}$. Since $\overline{t_{1}}$ is observed for this board, $T_{4}$ is then selected for diagnosis. As $t_{4}$ is obtained for this case, the root cause for this failing board is determined to be $A_{1}$.

\subsection{Diagnosis Using Incremental Decision Trees}

Diagnosis based on decision trees was used in the past even when DTs were generated manually by debug technicians [67]. Knowledge provided by experienced technicians were incorporated in DTs. However, these DTs cannot handle new error scenarios that become available during volume manufacturing. Technicians must regenerate the complete decision tree to incorporate the new knowledge. Decision trees can be generalized automatically using data analysis and splitting criteria described in the previous section. However, these automatically generated decision trees must also be

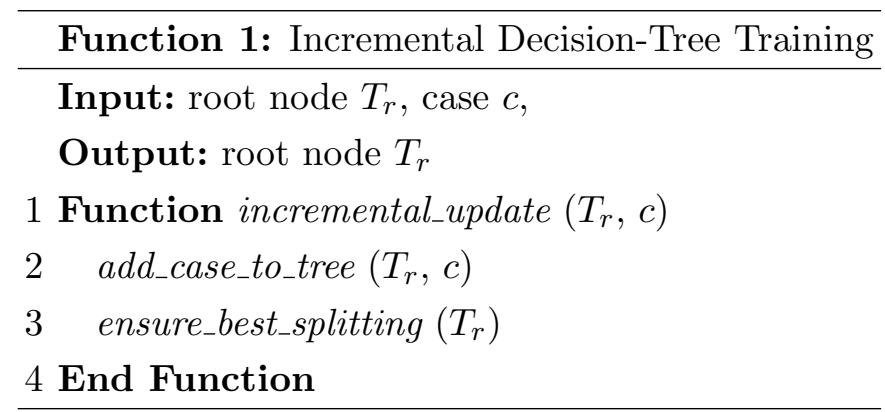

FIGURE 4.4: Outline of the training algorithm for iDTs. 


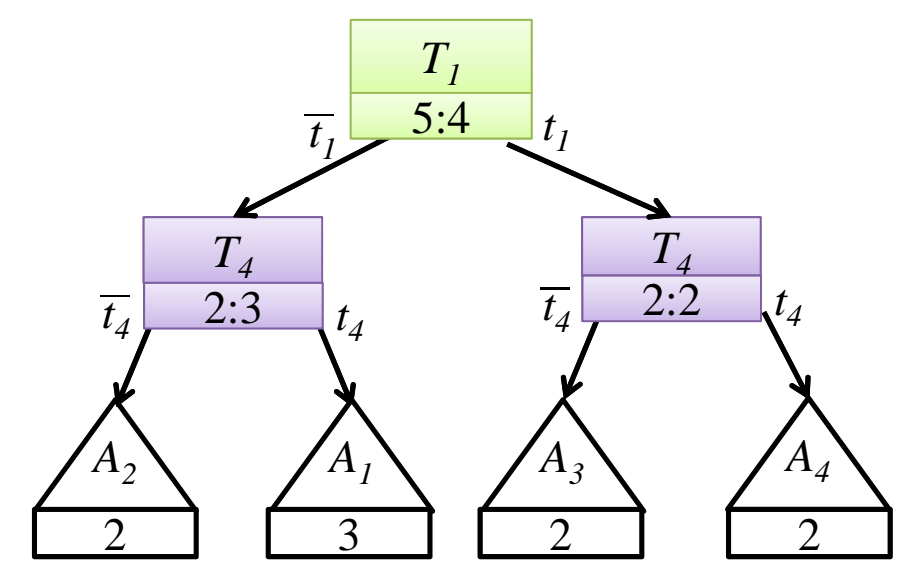

FiguRE 4.5: Illustration of nodes with additional scores for incremental learning.

retrained (regenerated) to deal with the occurrence of new errors and the resulting new knowledge. We tackle this problem using incremental decision trees (iDTs). The DT developed by technicians at test-design time can thus be incrementally and continuously updated to include new errors (Figure 4.1).

Figure 4.4 illustrates the procedure of training an incremental decision tree: 1) the decision tree is updated by incorporating the knowledge of a case using the procedure add_case_to_tree; 2) the tree is checked with the best splitting rule on each decision node recursively using the procedure ensure_best_splitting. We demonstrate the use of iDTs using the decision tree trained in Figure 4.3 based on the past logs. Note that the decision tree can also be derived by an experienced debug technician at the test-design stage.

\subsubsection{Incremental Tree Node}

To enable incremental learning in DTs, a tree node needs to maintain additional information. For each decision node, the counts of cases for each branch, namely scores, are also retained. Figure 4.5 shows the decision tree, in which all the decision nodes and leaf nodes are annotated with the corresponding scores. For example, the decision node of $T_{1}$ contains the score of 5:4, which means five cases have $\overline{t_{1}}$ 


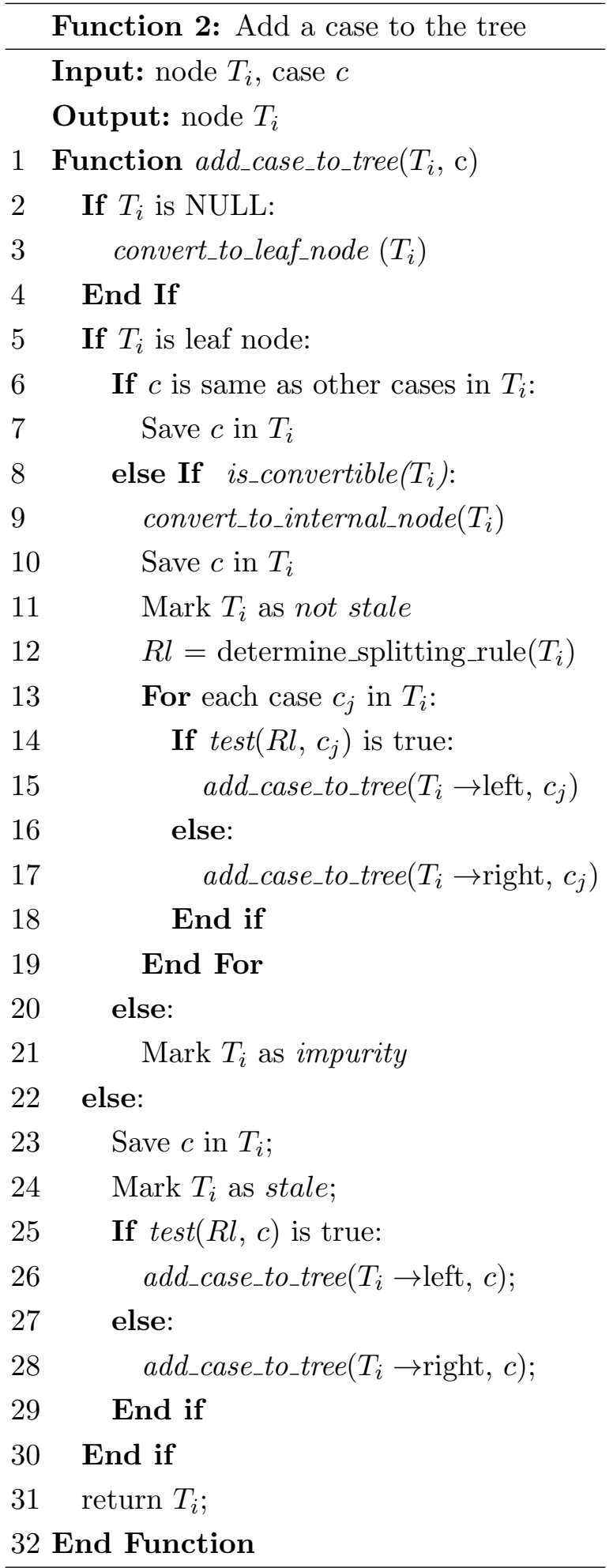

Figure 4.6: Procedure for adding a new case to a DT. 


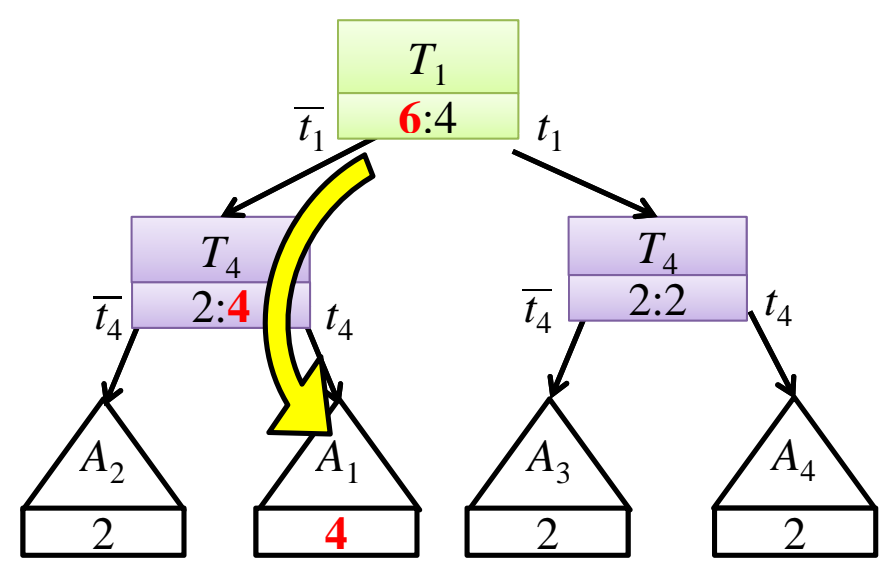

FIGURE 4.7: Illustration of the addition of a case (Scenario I).

derived from $T_{1}$, while the other four cases have $t_{1}$. The leaf node corresponding to $A_{1}$ contains the score of 3 , which means a total of three cases with the observation sequence $t_{1} \rightarrow \overline{t_{4}}$ have the root cause $A_{1}$. Here, we simply use the scores obtained from the board-repair database and Equation (4.4). Note that if the decision tree is generated by technicians, the scores stored in the tree nodes shall be assigned manually depending on the level of confidence of the technician.

\subsubsection{Addition of a Case}

Once a new case is available, the decision tree is updated by incorporating this case. The case is considered for inclusion in as many places as possible in the tree according to the branch rules in the decision nodes. The procedure in Figure 4.6 demonstrate the addition of a case in a tree. Three scenarios may occur during the addition of a new case.

In Scenario I (Figure 4.7), if the case has the same class label as the leaf node, it is simply added to the score stored in the leaf node.

For example, suppose we update the tree (Figure 4.5) using a case with the syndrome $\left[\begin{array}{llll}t_{1} & \overline{t_{2}} & t_{3} & t_{4}\end{array}\right]$ and the root cause $A_{1}$. The scores in the root node $T_{1}$, the decision node $T_{4}$ (left), and the leaf node $A_{1}$ are updated, which are highlighted in 


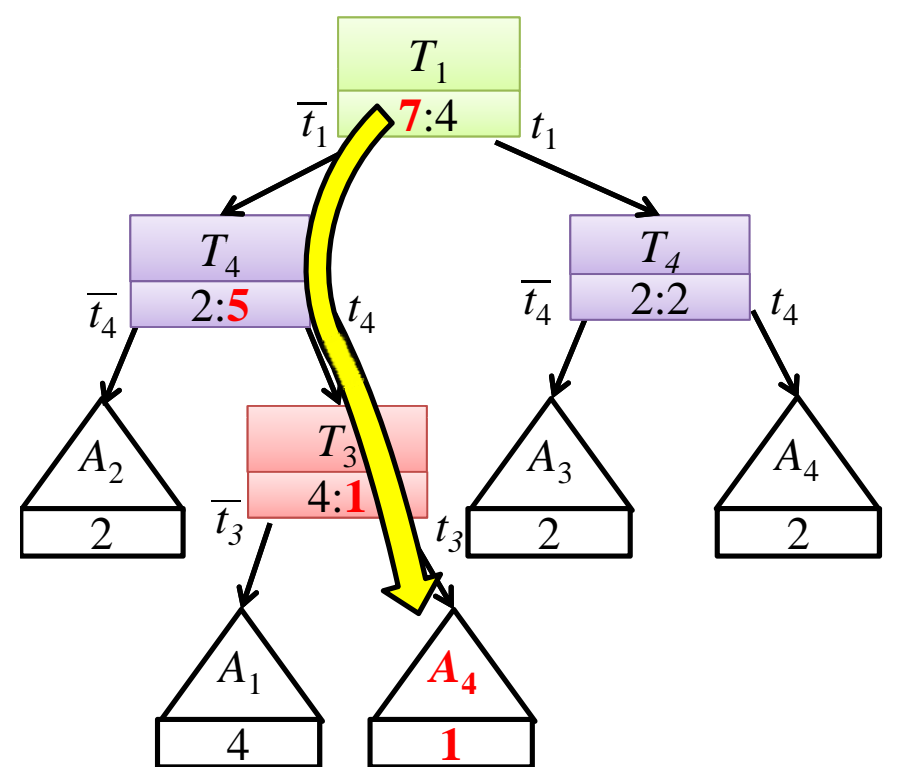

FiguRE 4.8: Illustration of the addition of a case (Scenario II).

the figure.

In Scenario II (Figure 4.8), if the new case has a different class label from the leaf node, the leaf node is converted to a decision node. All the cases in the previous leaf node are reorganized. They are transferred to the appropriate (new) leaf nodes according to the splitting rule on the new decision node. However, it is possible that one or more values of the cases can be missing during tree construction. To account for this possibility, a missing value is treated as a special value that does not satisfy the splitting rule at a decision node. In this work, a missing value for a syndrome $T_{i}$ is treated as $\overline{t_{i}}$.

For example, suppose we update the existing tree (Figure 4.7) using a new case with the syndrome $\left[\begin{array}{llll}t_{1} & \overline{t_{2}} & t_{3} & t_{4}\end{array}\right]$ and the root cause $A_{4}$. Following the tree path $\overline{t_{1}} \rightarrow t_{4} \rightarrow A_{1}$, the leaf node of $A_{1}$ is reached. Here, the new case with the root cause $A_{4}$ has a different class label from the leaf node of $A_{1}$. Hence, this leaf node shall be converted to a decision tree. Based on the missing-value rule, the values of $T_{2}$ and $T_{3}$ of the existing four cases in the prior leaf node are treated as $\overline{t_{2}}$ and $\overline{t_{3}}$, 
respectively. In the new case, $\overline{t_{2}}$ and $t_{3}$ are observed. Based on the information-gain criteria described in Section 4.2.1, individual information gains are calculated to be 0 for $T_{2}$, and 0.72 for $T_{3}$. Therefore, $T_{3}$ is selected to be the new decision node. The new tree is shown in Figure 4.8.

In Scenario III, if the new case has a different class label from the leaf node and the leaf node cannot be converted to a decision node, we keep the node as a leaf and marked it as an impurity leaf. The case will still be added to the score on the leaf node, but the case is marked with a different class label. The class label of the leaf node is determined by the majority class label with the most case-counts on the leaf node. For example, suppose we update the existing tree (Figure 4.8) using a new case $\left[\overline{t_{1}} \overline{t_{2}} \overline{t_{3}} t_{4}\right]$ and the root cause $A_{3}$. Following the tree path $\overline{t_{1}} \rightarrow t_{4} \rightarrow \overline{t_{3}} \rightarrow A_{1}$, the leaf node has a different class label from the actual root cause of the case. The incremental learning algorithm attempts to turn the leaf node to a decision node. Based on the missing-value rule, the values of $T_{2}$ of the existing cases are treated as $\overline{t_{2}}$, which is the same as the observation of $T_{2}$ on the new case. Thus, $T_{2}$ cannot be turned to a new decision node, while the remaining three syndromes have already served as the decision nodes. Therefore, the leaf node is marked as an impurity leaf. Since the number of cases with the class label $A_{1}$ is more than the rest of the class labels on this leaf node, this leaf node is still labeled with $A_{1}$.

\subsubsection{Ensuring the Best Splitting}

The addition of new cases results in the expansion of the decision tree. The set of cases that satisfied a previous splitting rule is not necessarily admissible for a new rule. Therefore, the tree must be checked with the best splitting rule on each decision node. One way to ensure that each decision node is using the best splitting rule is to visit every node and to carry out an explicit check. However, it is not practical to visit all the decision nodes for splitting-rule evaluation. A decision node and its 


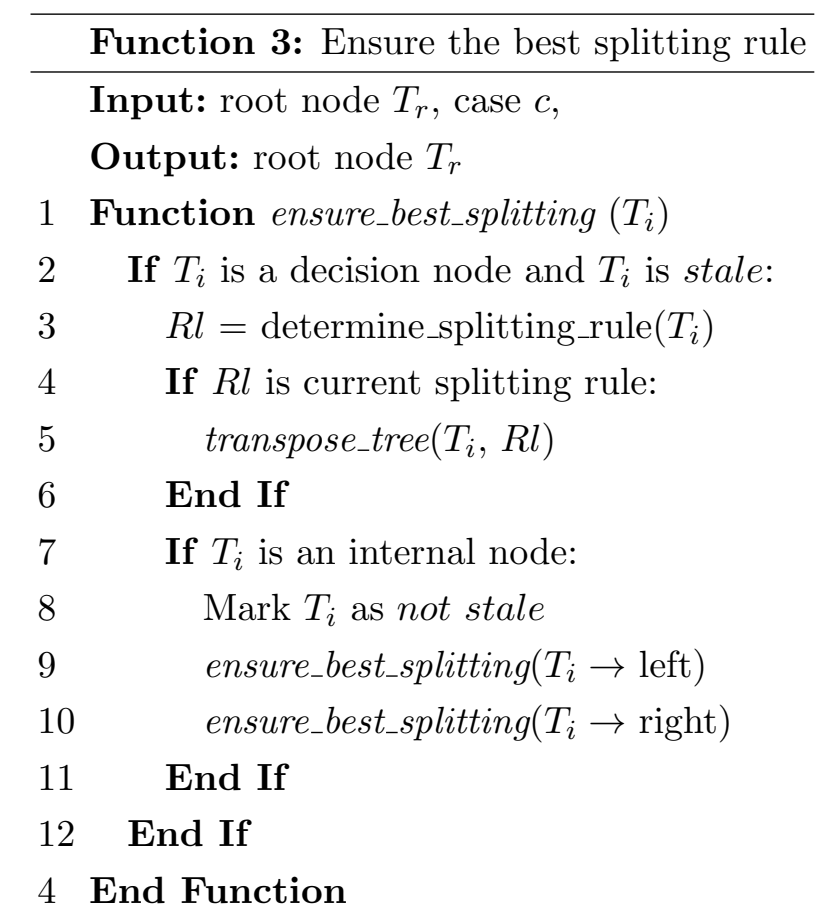

Figure 4.9: Pseudo-code for the algorithm ensure_best_splitting().

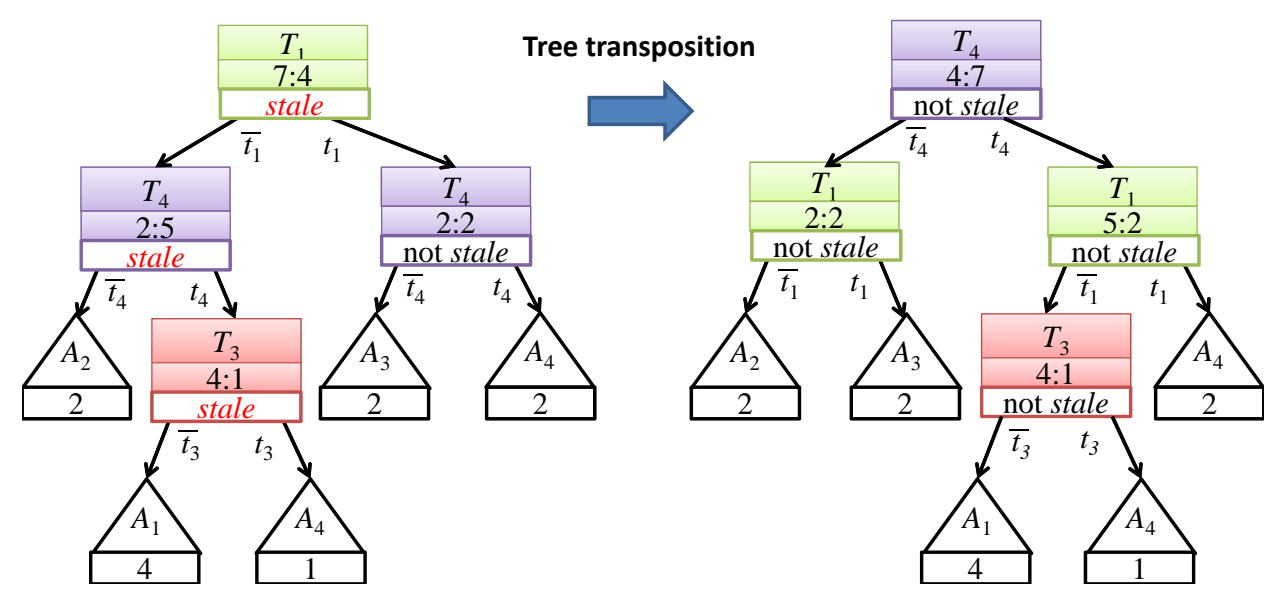

FigURE 4.10: Illustration of the tree-transposition procedure.

subtrees do not necessitate a change if no new cases are added to the node. A stale marker is thus defined to denote whether the score on a node is changed due to the addition of a case (see Figure 4.10). If a node is not stale, no change will be incorporated on the subtrees of the node. The use of a stale marker helps reduce the 
computation complexity of incremental training of a decision tree.

Figure 4.9 shows the procedure ensure_best_splitting. Consider the tree on the left side of Figure 4.10 as an example, we carry out the calculation of information gains for each syndrome, 1. 68 for $T_{1}, 0.45$ for $T_{3}$, and 1.93 for $T_{4}$, respectively. Therefore, the syndrome $T_{4}$ with the highest information gain is selected and pulled up to be the new root node. The child nodes of new root node $T_{4}$ must therefore be modified to adapt to the new rule, and recursive changes in the tree structure are necessary.

\subsubsection{Tree Transposition}

Rebuilding the entire tree can be prohibitively time-consuming and the past cases may no longer be available for rebuilding the DT. It is therefore desirable that the tree be reconfigured at low cost. This change can be accomplished through a sequence of recursive tree transpositions.

Consider the tree transposition on the decision tree as shown in Figure 4.10; a node of $T_{4}$ is elevated while a node of $T_{1}$ descends down the tree. Note that the only change is that the subtrees of two nodes of $T_{4}$ are reattached to the new nodes of $T_{1}$, thus the scores on the old nodes $T_{1}$ and $T_{4}$ are updated. The score of the left branch of the new node of $T_{4}$ is the sum of the scores of the left branches of the two previous nodes corresponding to $T_{4}$. Meanwhile, the score of the right branch of the new node of $T_{4}$ is the sum of the scores of the right branches of the two previous nodes corresponding to $T_{4}$. Moreover, there is no need to conduct tree-transposition on the node of $T_{3}$, since $T_{3}$ is still the best splitting rule on that decision node.

\subsection{Diagnosis Flow Based on Incremental Decision Trees}

The iDT-based diagnosis flow consists of the four steps shown in Figure 4.11. In general, a set of syndromes and corresponding repair actions are first defined by test 
designers. Next, an initial decision tree is derived for diagnosis. It can be either written by a test designer or trained from a board-repair database. The DT-based diagnosis system is ready for the diagnosis of new cases. Those new cases in turn can be used to update the diagnosis system.

Step 1: We first define the format of data, including inputs and outputs of DTs. This information is derived from board design and test design. The fault syndromes are defined and recorded in the log files for extraction as the inputs, and the corresponding repair component is taken as the output. Details of the datapreparation stage have been described in Section 4.1.

Step 2: In this step, we can either start from a blank tree, which has no diagnosis ability or start with an existing tree. The starting tree can be derived by an experienced test designer, who is familiar with the diagnosis procedure. The scores in each node can be assigned weights according to the confidence level of the test designer.

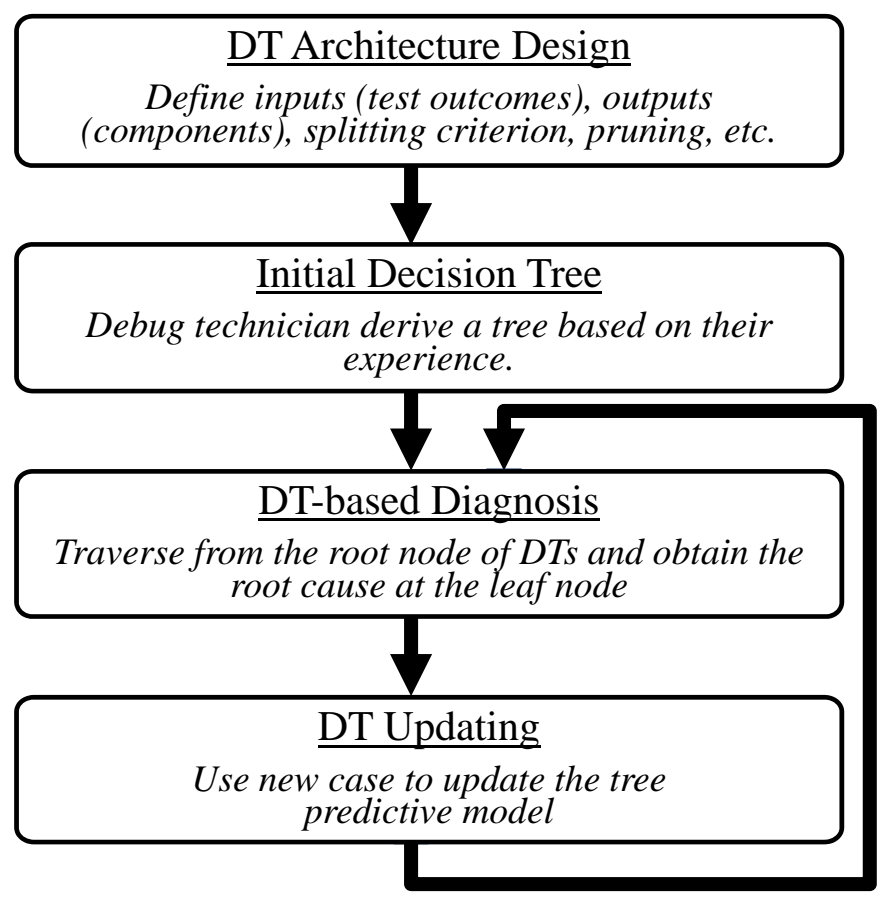

Figure 4.11: The diagnosis flow using DTs. 
If the test designer has a low level of confidence, he can assign lower weights and let the tree be updated via automated learning during volume manufacturing. In contrast, if the initial weights are set large, the decision tree is more rigid and hard to alter. We compare the different weight settings of a decision tree generated by test designers in Section 4.5.

Step 3: We can use the DT-based diagnosis system for new-case diagnosis. The new-case diagnosis procedure is shown in Figure 4.12. We start the diagnosis from the root node of the DT, which is the most discriminative syndrome. Based on the observation of this syndrome, we determine the next syndrome for diagnosis. The follow-up procedure is conducted in a heuristic and recursive manner in that the selection of a new syndrome is determined from the observated result of the current test. Eventually, a leaf node is reached after analysis of several syndromes as part of the DT that is traversed. We can predict the root cause based on the suggestion of the leaf node.

Step 4: The existing decision tree can be updated by incorporating the new cases. If a new case alters the decision tree based on the evaluation of the best-splitting rule, a tree transposition is necessary. The decision tree follows the procedure described in Section 4.3 to conduct a recursive tree transposition from top to bottom. The training procedure can be implemented using existing machine learning algorithms available in the Weka toolkit [58].

To ensure the accuracy of the proposed DT-based diagnostic system, we consider up to three attempts for identifying root-cause candidates for repair. A board must be scrapped after three unsuccessful attempts at repair. The first attempt corresponds to the first leaf node reached during diagnosis. When the first attempt fails to repair the board, we seek the second root-cause candidate either from the minority class labels in the impurity leaf node or from the sub-tree of the first attempt's parent node. This means that we assign an observation error on the parent node (syndrome) of 


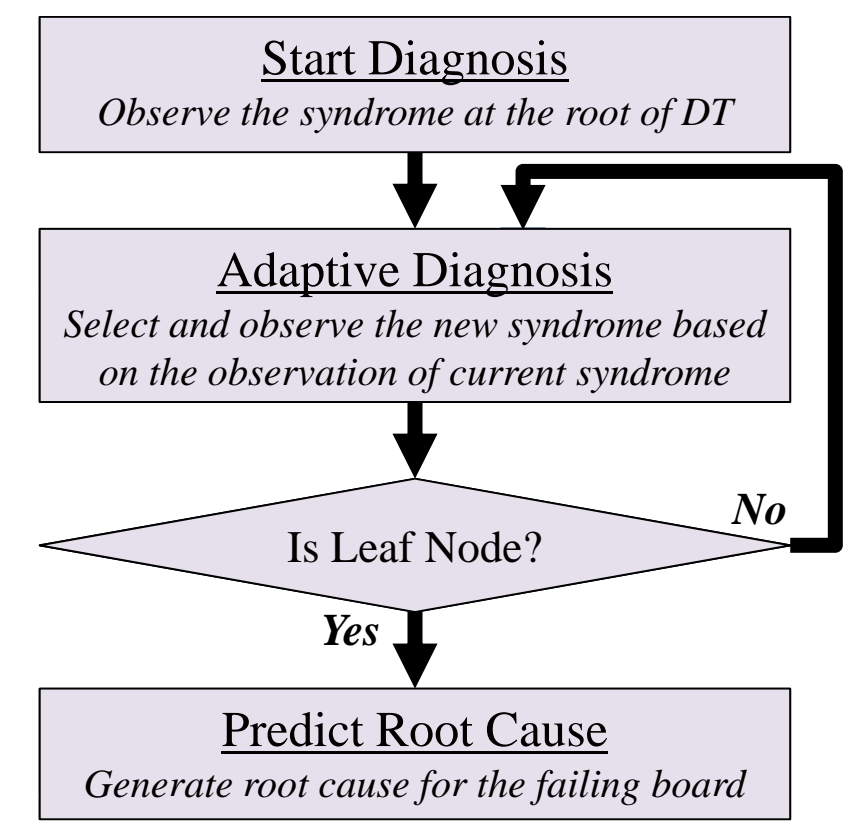

Figure 4.12: Diagnosis of new cases using DTs.

Table 4.1: Information about the industrial boards used for classification and the log data available.

\begin{tabular}{|c|c|c|}
\hline & Board 1 & Board 2 \\
\hline Number of syndromes & 546 & 375 \\
\hline $\begin{array}{c}\text { Number of repair } \\
\text { candidates (components) }\end{array}$ & 153 & 116 \\
\hline Number of boards & 1613 & 1351 \\
\hline
\end{tabular}

the first attempt since this parent node led to a wrong repair attempt. By continuing the diagnosis procedure using the DT, we will reach another leaf node corresponding to a root-cause candidate that is different from that for the first attempt. The third attempt follows the steps described above.

\subsection{Experiments and Results}

Experiments were performed on two industrial boards that are currently in highvolume production. Relevant information about the boards is provided in Table 2.1, 
Table 4.2: Comparison of SRs and number of syndromes obtained by ANNs, SVMs and DTs with different splitting criteria.

(a) Board 1

\begin{tabular}{|c|c|c|c|c|c|c|}
\hline \multicolumn{2}{|c|}{ Machine learning method } & $\mathrm{SR}_{1}$ & $\mathrm{SR}_{2}$ & $\mathrm{SR}_{3}$ & $n_{T}$ & $n_{A}$ \\
\hline \multirow{2}{*}{$\begin{array}{c}\text { Decision } \\
\text { trees }\end{array}$} & Gini index & $65.0 \%$ & $75.0 \%$ & $81.5 \%$ & 151 & 9 \\
& Info. gain & $65.0 \%$ & $75.0 \%$ & $82.5 \%$ & 155 & 9 \\
\hline Baseline & Twoing & $62.5 \%$ & $72.0 \%$ & $79.0 \%$ & 153 & 9 \\
methods & SVMs & $67.9 \%$ & $78.1 \%$ & $84.4 \%$ & 546 & 546 \\
& $73.2 \%$ & $80.4 \%$ & $88.2 \%$ & 546 & 546 \\
\hline
\end{tabular}

(b) Board 2

\begin{tabular}{|c|c|c|c|c|c|c|}
\hline \multicolumn{2}{|l|}{ Machine learning method } & $\mathrm{SR}_{1}$ & $\mathrm{SR}_{2}$ & $\mathrm{SR}_{3}$ & $n_{T}$ & $n_{A}$ \\
\hline \multirow{2}{*}{$\begin{array}{c}\text { Decision } \\
\text { trees }\end{array}$} & Gini index & $52.7 \%$ & $68.0 \%$ & $72.7 \%$ & 83 & 8 \\
& Info. gain & $55.3 \%$ & $69.0 \%$ & $72.7 \%$ & 83 & 8 \\
\hline \multirow{2}{*}{$\begin{array}{c}\text { Baseline } \\
\text { methods }\end{array}$} & Twoing & $52.7 \%$ & $68.0 \%$ & $73.7 \%$ & 83 & 8 \\
\hline
\end{tabular}

as reproduced below in Table 4.1 .

Algorithms were implemented using Matlab 2011b statistics toolbox [68]. Experiments were run on a 64-bit Linux system with 12 GB of RAM and quad-core Intel i7 processors running at $2.67 \mathrm{GHz}$. Diagnosis results were obtained for different designs of the DTs, e.g., for various splitting criteria, values of the pruning parameter. Diagnosis results for DT-based system using information gain as the splitting criterion and a relatively small value for the pruning parameter provides higher diagnosis accuracy and leads to fewer syndromes for diagnosis.

In this work, we use the same cross-validation methods and concepts of success ratios, as described in Chapter 2, to evaluate the performance of ANN-based, SVMbased, and DT-based diagnosis systems.

We first compare the number of syndromes $n_{T}$ used in DTs and average number of syndromes $n_{A}$ used for identifying a root cause using DTs to those using ANNs and 
Table 4.3: Comparison of DTs and SVMs with reduced set of syndromes.

(a) Board 1

\begin{tabular}{|l|c|c|c|c|c|}
\hline & DTs & SVMs $^{1}$ & SVMs $^{2}$ (min) & SVMs $^{2}$ (max) & SVMs $^{2}$ (avg.) \\
\hline $\mathrm{SR}_{1}$ & $65.0 \%$ & $73.2 \%$ & $35.5 \%$ & $54.7 \%$ & $43.9 \%$ \\
$\mathrm{SR}_{2}$ & $75.0 \%$ & $80.4 \%$ & $51.7 \%$ & $68.2 \%$ & $60.0 \%$ \\
$\mathrm{SR}_{3}$ & $82.5 \%$ & $88.2 \%$ & $65.8 \%$ & $78.3 \%$ & $72.8 \%$ \\
\hline
\end{tabular}

(b) Board 2

\begin{tabular}{|l|c|c|c|c|c|}
\hline & DTs & SVMs $^{1}$ & SVMs $^{2}$ (min) & SVMs $^{2}$ (max) & SVMs $^{2}$ (avg.) \\
\hline $\mathrm{SR}_{1}$ & $55.3 \%$ & $66.3 \%$ & $29.2 \%$ & $37.5 \%$ & $31.1 \%$ \\
$\mathrm{SR}_{2}$ & $69.0 \%$ & $74.3 \%$ & $43.3 \%$ & $61.7 \%$ & $56.8 \%$ \\
$\mathrm{SR}_{3}$ & $72.7 \%$ & $84.1 \%$ & $57.5 \%$ & $70.0 \%$ & $63.3 \%$ \\
\hline
\end{tabular}

${ }^{1}$ SVMs trained using syndromes from DTs. ${ }^{2}$ SVMs trained using randomly selected $n$ syndromes, where $n$ is the number of syndromes in the DT.

SVMs; see Table 4.2. As described in previous chapters, ANNs and SVMs use all the available syndromes for training and new-case diagnosis; the number of syndromes used for diagnosis is therefore equal to that used for training. In contrast, DTs require even fewer syndromes in diagnosis compared to the reduced number needed for training. For example, after DT training, the number of syndromes for diagnosis is reduced from 546 to 155 or less for Board 1 and from 375 to 83 for Board 2. Different splitting criteria lead to different structures of DTs and therefore different numbers of syndromes. For example, the number of syndromes used for training for Board 1 is 151 using Gini index, 155 using information gain, and 153 using Twoing. The average number of syndromes (average over all the failing boards) for identifying a root cause is only 9 for Board 1 and only 8 for Board 2 .

We next compare the SRs for DTs to that for SVMs and ANNs in Table 4.2. We find that the SRs for DTs remains significantly high even the number of syndromes used for diagnosis reduced by an order of magnitude for three boards. Our results show that we can identify a small subset of syndromes through DT training that has the same discriminative ability as the complete set of syndromes for diagnosis and 
Table 4.4: Comparison of DTs and ANNs with reduced set of syndromes.

(a) Board 1

\begin{tabular}{|l|c|c|c|c|c|}
\hline & DTs & ANNs $^{3}$ & ANNs $^{4}$ (min) & ANNs $^{4}$ (max) & ANNs $^{4}$ (avg.) \\
\hline $\mathrm{SR}_{1}$ & $65.0 \%$ & $67.9 \%$ & $16.3 \%$ & $23.8 \%$ & $19.0 \%$ \\
$\mathrm{SR}_{2}$ & $75.0 \%$ & $78.1 \%$ & $19.2 \%$ & $31.3 \%$ & $24.5 \%$ \\
$\mathrm{SR}_{3}$ & $82.5 \%$ & $84.4 \%$ & $25.5 \%$ & $41.7 \%$ & $29.1 \%$ \\
\hline
\end{tabular}

(b) Board 2

\begin{tabular}{|l|c|c|c|c|c|}
\hline & DTs & ANNs $^{3}$ & ANNs $^{4}$ (min) & ANNs $^{4}$ (max) & ANNs $^{4}$ (avg.) \\
\hline $\mathrm{SR}_{1}$ & $55.3 \%$ & $57.9 \%$ & $14.2 \%$ & $25.0 \%$ & $20.4 \%$ \\
$\mathrm{SR}_{2}$ & $69.0 \%$ & $70.3 \%$ & $19.7 \%$ & $33.3 \%$ & $24.2 \%$ \\
$\mathrm{SR}_{3}$ & $72.7 \%$ & $75.1 \%$ & $29.2 \%$ & $37.5 \%$ & $33.7 \%$ \\
\hline
\end{tabular}

${ }^{3}$ ANNs trained using syndromes from DTs. ${ }^{4}$ ANNs trained using randomly selected $n$ syndromes, where $n$ is the number of syndromes in the DT.

by ANNs or SVMs. We also find that the SRs are higher using Information Gain as the splitting criterion.

We next compare the SRs achieved using DTs with that achieved using the ANN and SVM baseline methods, when the set of syndromes is the same for all these methods. The results are shown in Table 4.3 and Table 4.4. The sets of syndromes selected by DTs are used for training SVM and ANN models. We also train the SVMs $(\mathrm{ANNs})$ with randomly selected syndromes, such that the number of syndromes in the SVMs (ANNs) is same as in the DTs. Random selections are repeated 20 times. The maximum, minimum, and average SRs obtained are listed in Table 4.3 and Table 4.4. These results show that for the same small number of syndromes, DT outperforms ANNs and SVMs in nearly all cases. Moreover, DTs can be used to select the most discriminative syndromes for use by ANNs and SVMs - as a result, the success ratio is often higher than when a much larger number of syndromes is used.

Next, we evaluate the benefit of using incremental learning in diagnosis systems. In Figure 4.13-4.14, we compare the diagnosis accuracies of four diagnosis systems. 


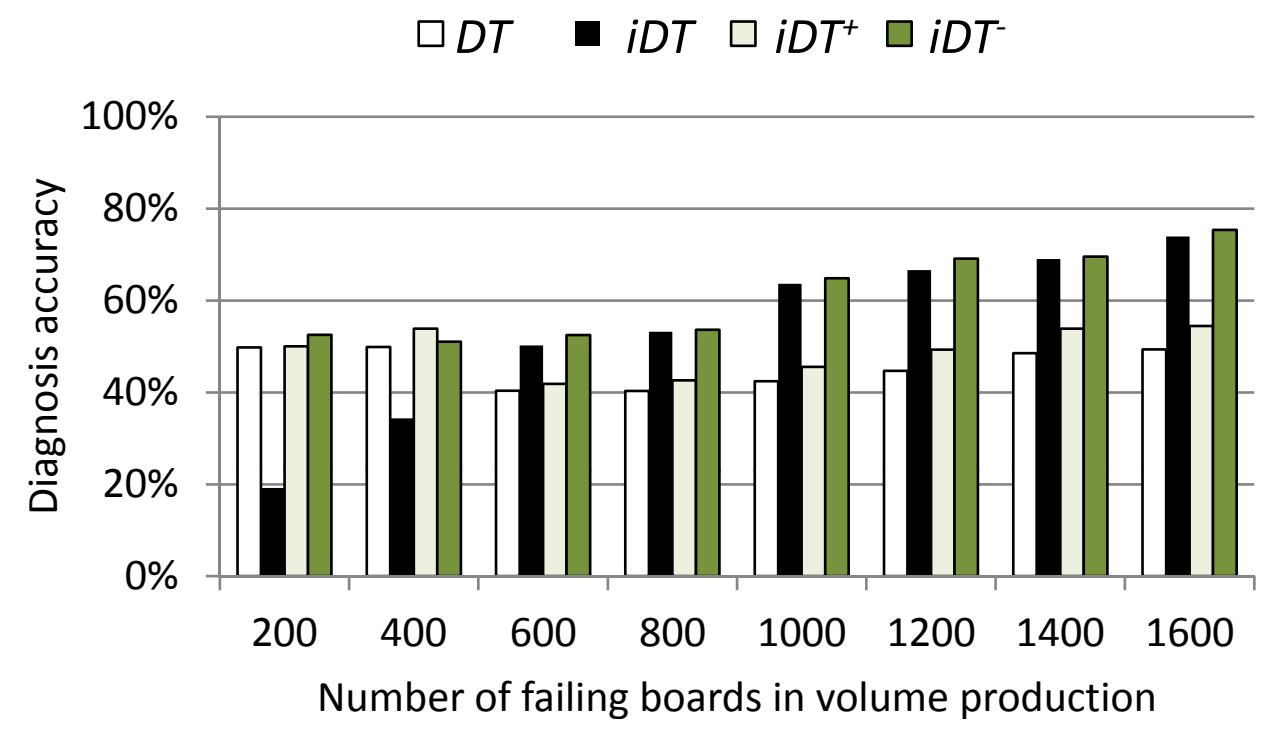

Figure 4.13: Comparison of fault diagnosis using batch DTs, incremental DTs, incremental DTs* for Board 1.

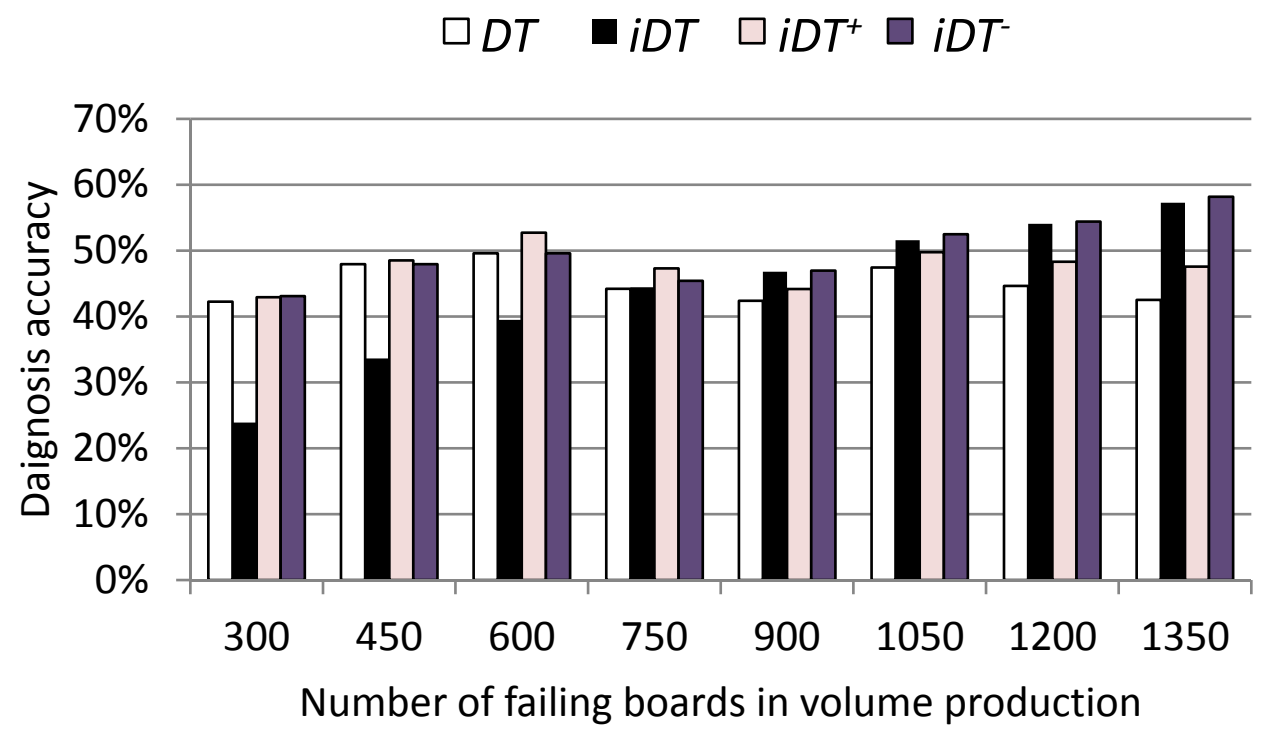

Figure 4.14: Comparison of fault diagnosis using batch DTs, incremental DTs, incremental DTs* for Board 2.

$D T$ is a diagnosis system based on the DT written by the debug technicians. The diagnosis system is non-incremental. iDT is a diagnosis system based on an incremental DT, but the DT starts with an empty tree at the beginning of volume 
manufacturing. $i D T^{+}$and $i D T^{-}$are two iDT-based diagnosis systems with initial DT derived from an experienced technician. The difference is that the scores of all leaf nodes in $i D T^{+}$are initially assigned high (e.g., 50), while those of $i D T^{-}$are assigned low scores (e.g., 1). We observe that the diagnosis accuracies of the DTs, i.e., $\mathrm{SR}_{1}$ are approximately $40 \%-50 \%$ on average, which is common in modern product lines. As the product volume increases, the diagnosis accuracy does not increase, but even drops sometimes. iDT shows very low diagnosis ability at the beginning of volume production, but the diagnosis accuracy ramps up gradually due to incremental learning as the product volume accumulates. Next, we observe that the diagnosis accuracies of $i D T^{-}$and $i D T^{+}$are significantly better. At the beginning, the performances of $i D T^{-}$and $i D T^{+}$are close to $D T$, and then the diagnosis accuracies increase as more boards are available for learning. Note that $i D T^{-}$performs much better than $i D T^{+}$in all three diagnosis systems. The reason is that $i D T^{-}$is easier to adapt to the knowledge of new error scenarios.

\subsection{Conclusions}

This chapter presented an adaptive board-level functional fault diagnosis method based on incremental decision trees (iDTs). It is shown that how faulty components can be classified based on the discriminative ability of the syndromes in DT training. The diagnosis procedure has been constructed as a binary tree, with the most discriminative syndrome as the root and the final repair suggestions are available as the leaf nodes of the tree. In this way, accurate diagnosis can be carried out at low cost with a small number of syndromes. Furthermore, we have combined the diagnosis knowledge from debug technicians with the knowledge gained from failing boards during volume manufacturing. Therefore, the problem of low diagnosis accuracy at the beginning of volume production can be alleviated. The diagnosis system can adapt to occurrences of new error scenarios to enhance diagnosis accuracy on- 
the-fly. Diagnosis results for two complex boards from industry, currently in volume production, highlight the effectiveness of the proposed approach. 


\section{5 \\ Handling Missing Syndromes}

\subsection{Introduction}

The diagnosis accuracy of reasoning-based diagnosis engine may be significantly reduced when the repair logs are fragmented and some errors, or syndromes, are not available during diagnosis. Since root-cause isolation for a failing board relies on reasoning based on syndromes, any information loss (e.g., missing syndromes) during the extraction of a diagnosis log may lead to ambiguous repair suggestions.

In this chapter, we propose a board-level diagnosis system with the feature of handling missing syndromes using the method of imputation. The syndromes from a faulty-board log are analyzed and imputed with appropriate values in a preprocessing engine before root-cause isolation. We utilize several imputation methods and compare them in terms of their effectiveness in handling missing syndromes.

The remainder of this chapter is organized as follows. Section 5.2 discusses the missing-syndrome problem in more detail and highlights the contributions of this chapter. Section 5.3 discusses the effects of missing syndromes on a diagnosis system. A number of imputation techniques are used to extend the current diagnosis system 
to handle missing syndromes. In Section 5.4, experimental results on two large-scale synthetic data sets, generated from complex boards in high-volume production, are used to demonstrate the effectiveness of the proposed diagnosis system in terms of diagnosis accuracy and training time. Finally, Section 5.5 concludes the chapter.

\subsection{Problem Statement and Chapter Contributions}

Unlike chip-level fault diagnosis, which can be automated via ATE and advanced debug/diagnosis tools [10], board-level diagnosis still requires a considerable amount of manual expertise. Recent work on board-level fault diagnosis has shown that machine-learning techniques can be adopted to automate the process of identifying faulty candidates (components) based on the historical data of successfully repaired boards $[2,40]$.

A diagnostic system based on machine learning does not need to understand the complex functionality of boards, and it is able to automatically derive and exploit knowledge from the repair logs of previously documented cases. The extraction of fault syndromes, i.e., test outcomes, is critical for model training in a diagnosis system. The fault syndromes should provide a complete description of the failure, and the extracted syndromes for different actions should have sufficient diversity such that we can eliminate ambiguity in the eventual repair recommendations. Fault syndromes vary across products and tests. For example, a segment of the log file for a failed traffic test is shown in Figure 5.1(a). The fault syndromes extracted are R3d3-metro (counts mismatch), LA0 Engine 0x0000ffff (error counter), and 0x11 (error code). Each of these elements is considered to be one syndrome. The repair

action is often directly recorded in the database, e.g., "replacement of component U11". All the extracted syndromes and actions are used for training of the proposed diagnosis system.

In prior work, e.g., in [40], functional-test logs were parsed in a coarse manner 


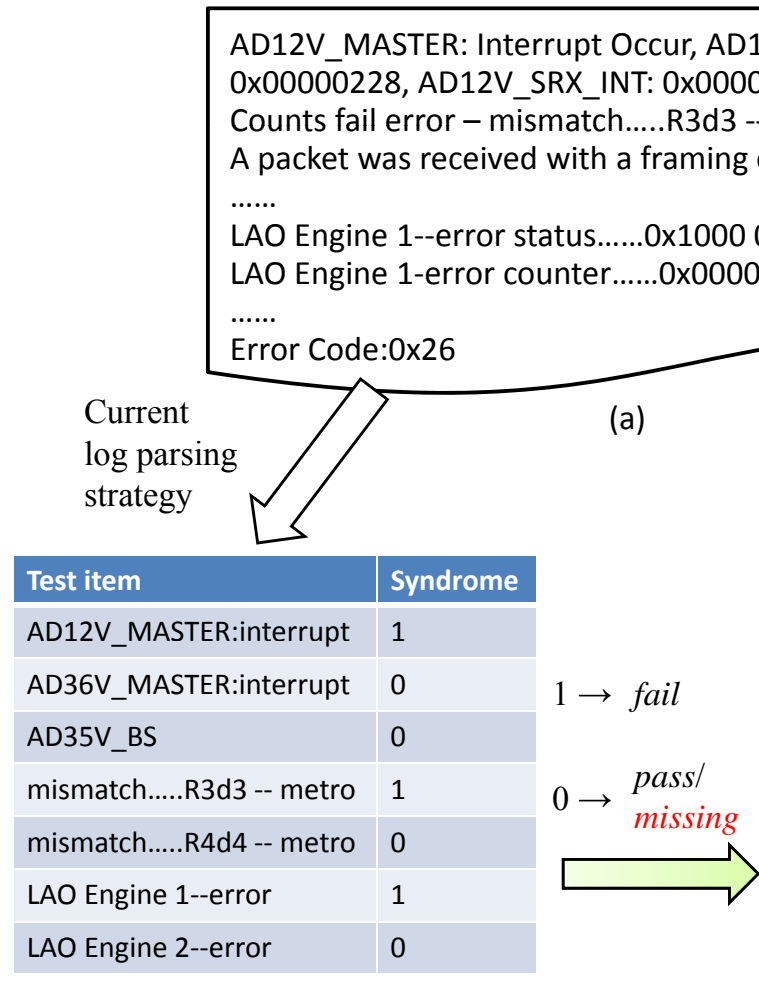

(b)

CX00000228, AD12V_SRX_INT: 0x00000002,

LAO Engine 1--error status......0x1000 000011

Engine 1-error counter......0x0000 0fff

Error Code:0x26

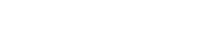

(a)

\begin{tabular}{|l|l|}
\hline Test item & Syndrome \\
\hline AD12V_MASTER:interrupt & fail \\
\hline AD36V_MASTER:interrupt & missing \\
\hline AD35V_BS & pass \\
\hline mismatch.....R3d3 -- metro & fail \\
\hline mismatch.....R4d4 -- metro & pass \\
\hline LAO Engine 1--error & fail \\
\hline LAO Engine 2--error & missing \\
\hline
\end{tabular}

(c)

FiguRE 5.1: Illustration of parsing a log with and without missing syndromes.

where a syndrome is 1 (fail) if a functional test leads to an error at the corresponding observation point. All other functional tests and observation-point pairs, which are mapped to syndromes, are deemed as 0 (pass); see Figure 5.1(b) [3,40,41]. However, a syndrome should not be denoted as either fail or pass when the observation of the test is not available in practice. We propose to define a third syndrome, namely missing syndrome, when the syndrome for a (test, observation point) pair is not observed (see Figure 5.1(c)). There are two scenarios that lead to the absence of observation of a syndrome:

1) Systematic missing syndromes: In current test-program design methods, the tasks of designing functional tests are divided into multiple packages and dispatched to multiple design teams [5]. Functional test packages are executed sequentially on a board-under-test. There is a likelihood that the execution of functional tests in 
later packages is affected by tests in early packages. For example, a functional test, mainboost, is used to check the functionality of a global controller, which is required to enable the functional tests that follow [5]. If the controller fails or the attached DRAMs fail, the diagnosis procedure is terminated and subsequent tests cannot be executed. The syndromes corresponding to these tests are, therefore, not available to the diagnosis system.

2) Random missing syndromes: Another scenario in current diagnosis systems is that a few functional tests may occasionally fail to record syndromes. The reason for these occasional syndrome escapes may not be due to board failure, but because of program bugs in functional test design $[2,5]$. Such bugs may either lead to incorrectly recorded syndromes or missing observations in the log. In these scenarios, the diagnosis system should be designed to be able to reason in the presence of these missing syndromes.

Both of the above scenarios lead to a decrease in the total number of syndromes available for diagnosis, thus resulting in low diagnosis accuracy. In addition, machine learning-based diagnosis systems proposed in the literature are not equipped to deal with these missing values $[3,4,25]$. These diagnosis systems are not robust to data sets with missing syndromes.

We therefore address the important practical problem of handling missing syndromes in a diagnosis system. The proposed solution preprocesses diagnostic logs based on value imputations; the details are presented in Section 5.3. We propose several imputation methods to handle missing values in an effective manner. First, a number of numerical imputation methods are used, such that complete analysis is carried out with the data set of faulty boards, and weighted estimated values are subsequently used to impute missing syndromes. Next, an alternative label imputation method is presented in which an additional feature missing is added to explicitly label whether a syndrome is missing. 


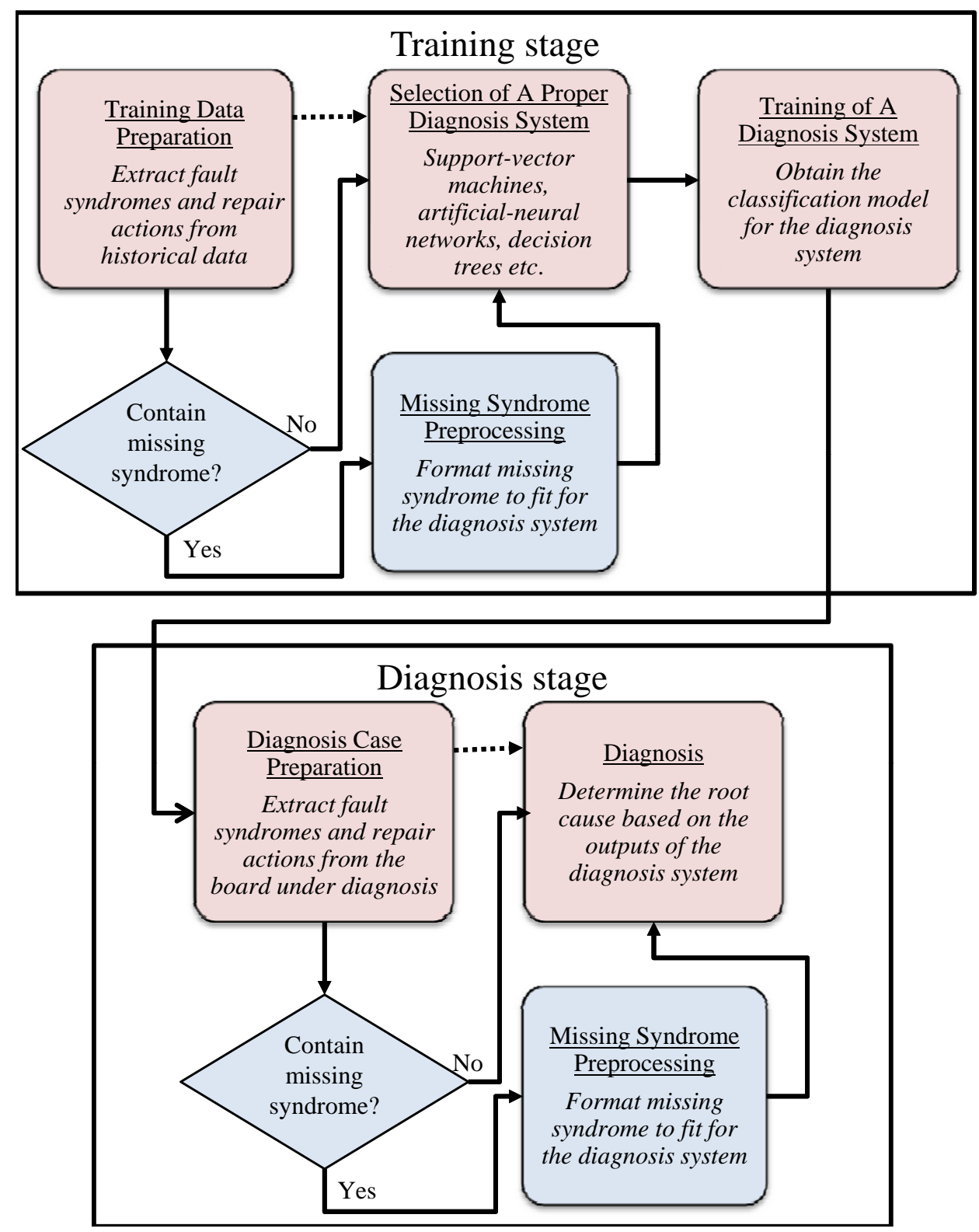

Figure 5.2: The SVM-based diagnosis flow with the capability of handling missing syndromes.

\subsection{Methods to Handle Missing Syndromes}

Missing data frequently occur in applied data analysis $[69,70]$. As described in Section 5.2, several scenarios may lead to missing syndromes, e.g., resulting in system- 
atic missing syndromes and random missing syndromes. To address the problem of missing syndromes, a non-convex max-margin formulation is used in classical SVM classifiers [70]. However, this method suffers from prohibitively high computation complexity, which makes it infeasible for board diagnosis in realistic scenarios. An alternative method, referred to as imputation, is to fill the missing syndromes in the data preprocessing stage $[69,71]$. Imputation methods are widely used because of their simplicity and ease of implementation. Imputation based on statistical central tendency (e.g., mode, median, or mean) has often been used in machine learning to treat missing values [71]. The K-nearest neighbor (KNN) and sample mean imputation (SMI) have also been used to carry out the imputation of missing data [72].

A reasoning-based diagnosis flow in [40] can be improved by integrating the component of preprocessing missing values, as shown in Figure 5.2. A revised diagnosis flow that is capable of handling missing syndromes can be described as follows. Once training data are extracted from diagnosis logs, the diagnosis system determines whether the data set contains missing syndromes, i.e., by comparing the number of obtained syndromes and total number of designed syndromes. If no missing syndrome is detected, the diagnosis system continues with the standard diagnosis-system training. Otherwise, the training data set is handled by the missing-value-preprocessing component. The processed data set is then sent for training of the diagnosis system. The same procedure also applies to the new failing boards under diagnosis. The preprocessed new cases are sent to the diagnosis system for root-cause isolation.

To address the missing syndromes, a number of imputation methods can be used $[69,71]$. These methods can be categorized into two main categories, namely numerical imputation and label imputation. 


\subsubsection{Numerical Imputation}

Numerical imputation is widely used to infer missing values. We continue to denote fail syndrome as 1 and pass syndrome as 0 . Missing value is predicted on a numerical scale based on analysis of the failing boards. Several imputation methods, e.g., mean and K-nearest neighbor (KNN), can be used $[69,71]$ and they are described in detail later. Consider a set of faulty $\operatorname{logs} \mathcal{M}$ with missing syndromes, and let $x_{1}, x_{2}$ and $x_{3}$ be three syndromes. Let us suppose that the board has two candidate root causes $\mathrm{A}$ and $\mathrm{B}$, and we encode them as $y=-1$ and $y=1$, respectively. Here, we merge the syndromes and the known root causes into one matrix $\mathcal{M}=[\mathcal{B} \mid \mathcal{C}]$, where the left $(\mathcal{B})$ side refers to syndromes, while the right side $(\mathcal{C})$ refers to the corresponding fault classes. This matrix represents the training information for a diagnosis system,

$$
\mathcal{M}=\left[\begin{array}{ccc:r}
1 & b_{21} & 0 & 1 \\
b_{12} & 1 & 1 & 1 \\
1 & 1 & 0 & 1 \\
b_{14} & 1 & 1 & -1 \\
0 & 0 & 1 & -1 \\
0 & 0 & b_{36} & -1
\end{array}\right]
$$

where $b_{i j}$ is the missing value of syndrome $x_{i}$ collected from the failing board $j$. Different imputation methods can be used to assign values for $b_{i j}$; these are discussed below.

\section{Zero Imputation}

In the prior log parsing strategy in Figure 5.1(b), all missing syndromes are deemed to be pass, which is denoted as 0 . Zero imputation follows the same imputation method, where any missing syndrome $b_{i j}$ is imputed with 0 . As an illustration, 
Equation (5.1) can be revised with zero-imputation values, as shown below:

$$
\mathcal{M}=\left[\begin{array}{lll:r}
1 & 0 & 0 & 1 \\
0 & 1 & 1 & 1 \\
1 & 1 & 0 & 1 \\
0 & 1 & 1 & -1 \\
0 & 0 & 1 & -1 \\
0 & 0 & 0 & -1
\end{array}\right]
$$

where italics indicate the imputed values and are used to denote imputed values throughout this chapter.

\section{Mean-Value Imputation}

Assume that $A_{i}$ is the complete set of entries for syndrome $x_{i}$, and $A_{i}^{\prime}$ is the subset of $A_{i}$ that contains all the non-missing entries, where $a_{i j} \neq$ missing and $a_{i j} \in A_{i}$. In this method, a mean value $b_{i j}$ is computed based on $A_{i}^{\prime}$, such that the imputation value $b_{i j}$ can be regarded as the probability that the syndrome $x_{i}$ is fail. The imputation value $b_{i j}$ is calculated as shown below:

$$
b_{i j}=\operatorname{mean}\left(A_{i}^{\prime}\right)=\frac{\sum_{a_{i j} \in A_{i}^{\prime}} a_{i j}}{\left|A_{i}^{\prime}\right|},
$$

where the imputation value $b_{i j}$ is same for each syndrome $x_{i}$. As an illustration, the corresponding matrix for the synthetic data is updated as:

$$
\mathcal{M}=\left[\begin{array}{ccc:c}
1 & 0.6 & 0 & 1 \\
0.5 & 1 & 1 & 1 \\
1 & 1 & 0 & 1 \\
0.5 & 1 & 1 & -1 \\
0 & 0 & 1 & -1 \\
0 & 0 & 0.6 & -1
\end{array}\right]
$$

\section{K-Nearest Neighbor Imputation}

Missing values are imputed with the mean value obtained from the $\mathrm{K}$ nearestneighbor instances. The neighborhood between instances is measured using the Euclidean distance. Let $E$ be an Euclidean distance matrix, where each entry $e_{p q}$ in $E$ 
represents the difference of features, including syndromes and root causes, between Board $p$ and Board $q$ based on the Euclidean-distance metric. The parameter $e_{p q}$ is calculated as shown below:

$$
e_{p q}=\sqrt{\sum_{i=1}^{|A|}\left(a_{p i}-a_{q i}\right)^{2}}
$$

where $a_{p i}$ and $a_{q i}$ represent the entries for syndrome $x_{i}$ in Board $p$ and Board $q$, respectively, and $|A|$ is the total number of fault syndromes. Assume that $K_{j}$ is the set of $\mathrm{K}$ nearest-neighbor instances for board $j$. The imputation value $b_{i j}$ is calculated as follows:

$$
b_{i j}=\operatorname{mean}\left(K_{j}\right)=\frac{\sum_{a_{i j} \in K_{j}} a_{i j}}{\mathrm{~K}} .
$$

Note that any missing syndrome in $K_{j}$ is simply considered to be 0 . If we set $\mathrm{K}$ $=2$, the corresponding imputed synthetic data is updated as follows:

$$
\mathcal{M}=\left[\begin{array}{ccc:r}
1 & 0.5 & 0 & 1 \\
0 & 1 & 1 & 1 \\
1 & 1 & 0 & 1 \\
0 & 1 & 1 & -1 \\
0 & 0 & 1 & -1 \\
0 & 0 & 0.6 & -1
\end{array}\right]
$$

\section{Hybrid Imputation}

Considering the difference between historical data for training (with root-cause information) and new failing boards for diagnosis (without root-cause information), we apply different imputation methods at the training stage and the diagnosis system. In this work, we use a diagnosis system based on SVMs, as in [40], to achieve higher diagnosis accuracy. At the system training stage, we discard the cases with missing syndromes from the training set, since an advantage of SVMs is that their diagnosis

accuracy depends on the support vectors rather than the size of training set [50]. At 
the diagnosis stage, we use mean-value imputation to gain as much information as possible. The main advantage of using hybrid imputation in a SVM-based diagnosis system, as evident from out results in Section 5.4, is high diagnostic accuracy and low training time.

\subsubsection{Label Imputation}

Let us revisit Figure 5.1(b), which shows the parsing strategy used in prior work. The numerical format of syndromes is widely used in machine learning and is convenient for representing binary syndromes. However, when we introduce a third syndrome, this binary format is no longer sufficient to describe the observations in the log of functional tests. Therefore, we propose to use another label missing to denote the absence of a syndrome; see Figure 5.1(c). Equation (5.1) can now be updated as shown below:

$$
\mathcal{M}=\left[\begin{array}{ccc:r}
\text { fail } & \text { missing } & \text { pass } & 1 \\
\text { missing } & \text { fail } & \text { fail } & 1 \\
\text { fail } & \text { fail } & \text { pass } & 1 \\
\text { missing } & \text { fail } & \text { fail } & -1 \\
\text { pass } & \text { pass } & \text { fail } & -1 \\
\text { pass } & \text { pass } & \text { missing } & -1
\end{array}\right]
$$

Machine-learning techniques can handle label syndromes by converting labels to numerical values [69]. For a trinary syndrome, two values are usually used to represent one syndrome. The first value $a_{i j}$ still denotes the failure observed in a syndrome, such that it is 1 if a syndrome is observed as fail, and 0 otherwise. The second value $a_{i j}^{\prime}$ denotes the occurrence of a syndrome, such that a missing syndrome is denoted with a weighted value $d$, and 0 otherwise. The reason for using these weighted values is that the additional feature $a_{i j}^{\prime}$ may mask the relationship between the original syndrome-fault pair. Fault syndromes are usually the dominant features for root-cause isolation, while missing syndromes are the secondary features. 
Table 5.1: Information about the synthetic boards used for classification.

\begin{tabular}{|c|c|c|}
\hline & Board 1 & Board 2 \\
\hline Number of syndromes & 546 & 375 \\
\hline Number of root causes & 153 & 116 \\
\hline Number of boards & 5000 & 5000 \\
\hline
\end{tabular}

Therefore, it is necessary to select an appropriate value $d$ in label imputation. The updated synthetic board is as follows:

$$
\mathcal{M}=\left[\begin{array}{cccccc:r}
1 & (0) & 0 & \left(d_{21}\right) & 0 & (0) & 1 \\
0 & \left(d_{12}\right) & 1 & (0) & 1 & (0) & 1 \\
1 & (0) & 1 & (0) & 0 & (0) & 1 \\
0 & \left(d_{14}\right) & 1 & (0) & 1 & (0) & -1 \\
0 & (0) & 0 & (0) & 1 & (0) & -1 \\
0 & (0) & 0 & (0) & 0 & \left(d_{36}\right) & -1
\end{array}\right]
$$

where $d_{i j}$ is the weighted value for the $j^{\text {th }}$ fault syndrome of the $i^{\text {th }}$ instance. We use the following weighted mean-value imputation to calculate $d_{i j}$,

$$
d_{i j}=\frac{\operatorname{mean}\left(A_{j}^{\prime}\right)}{w}=\frac{\sum_{a_{i j} \in A_{i}^{\prime}} a_{i j}}{w\left|A_{i}^{\prime}\right|}
$$

where $w$ is a single weight for tuning the feature of missing syndromes, and it is the same for imputing all syndromes. Choosing an appropriate value of $w$ is important for a diagnosis system in order to achieve high diagnosis accuracy. We provide guidelines for selecting $w$ in Section 5.4.

\subsection{Experiments and Results}

Experiments were performed on two synthetic boards that are extrapolated from real board designs shown previously in Table 2.1. As shown in Table 5.1, for Board 1, a total of 546 fault syndromes are extracted from failure logs. The number of faulty components identified in the database for repair actions is 153. A total of 375 syndromes and 116 faulty components are extracted for Board 2. 
The synthetic data sets are generated from two actual board designs because only a small number of faulty boards are available in the current early phase. For each root cause, we apply regression to every syndrome, thereby generating a probability based on the occurrence of the syndrome (i.e., the syndrome with more 1's in a class will be assigned a higher probability, while a lower probability indicates that it has more 0's). Based on these probabilities, we insert the corresponding variance values to the synthetic data (i.e., the syndrome with more 1's in the original data will also have more 1's in the synthetic data). We generated 5000 new cases for both Board 1 and Board 2, respectively.

All the algorithms are implemented in an open-source SVM toolkit, namely LibSVM [73]. Experiments were run on a 64-bit Linux system with 12 GB of RAM and quad-core Intel i7 processors running at $2.67 \mathrm{GHz}$.

To evaluate the diagnosis performance of the SVM-based learning system, we use a 5-fold cross-validation method [50], which randomly partitions the training set into 5 groups. Each group is regarded as a test case while all of the other cases are fed for training. The success ratio (SR) is the ratio of the number of correctly diagnosed cases to the total number of cases in the testing set. For example, if 10 instances are tested, SR of $70 \%$ means that 7 out of 10 cases are correctly classified. Here, we use a linear-kernel SVM-based diagnosis system, since its diagnosis performance has been shown to be better than SVM-based diagnosis systems based on other kernels. A comparison between SVM-based diagnosis systems using different kernels can be found in [40].

To evaluate the performance of the proposed imputation methods for handling missing syndromes, we generate random missing syndromes in our training data. Let $T$ be the entire set of training set, $S$ be the total number of syndromes, and $C$ be the total number of failing boards. Let $X$ be the total number of missing syndromes in $T$. The missing rate $(\mathrm{MR})$ is defined as $\frac{X}{S \times C}$. In this work, we use four types 


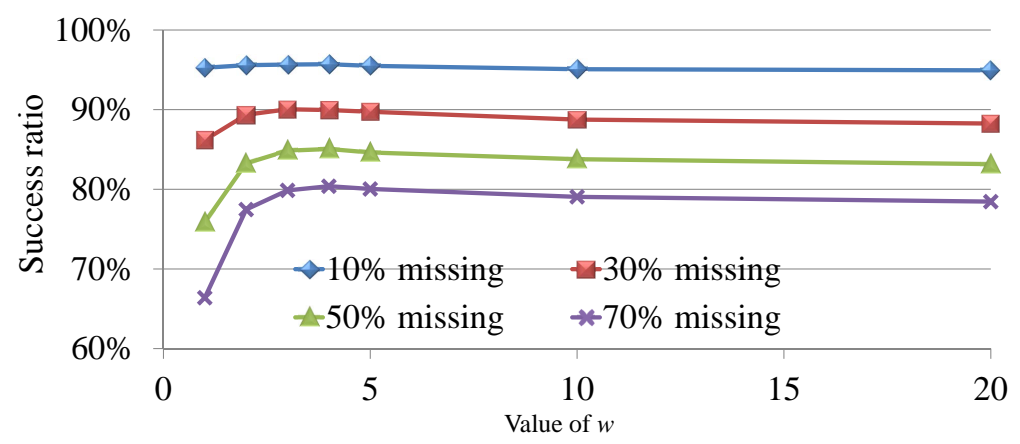

Figure 5.3: Comparison of success ratios for diagnosis using label imputation with different weights for Board 1.

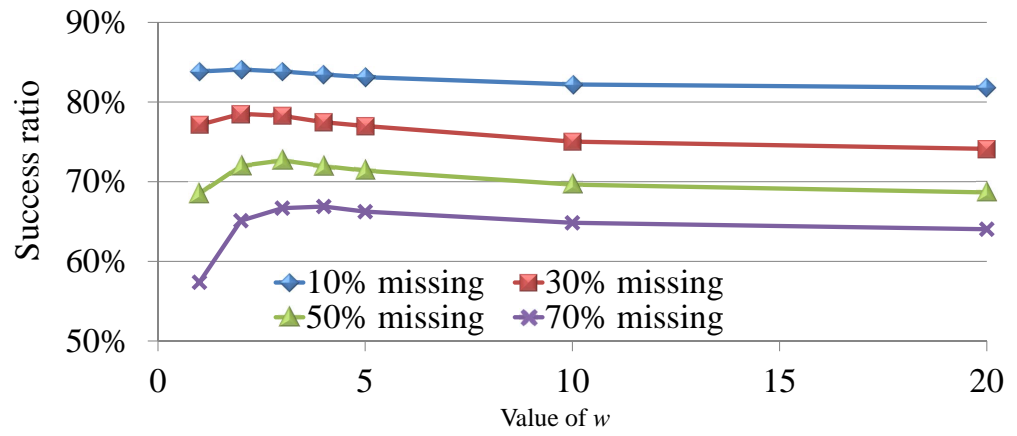

Figure 5.4: Comparison of success ratios for diagnosis using label imputation with different weights for Board 2.

of missing rates, $10 \%, 30 \%, 50 \%$, and $70 \%$, to consider a wide range of scenarios. We carried out 500 Monte Carlo simulation runs and the average success ratios are presented to show the diagnosis performance with different imputation methods. We report results for 500 runs because there was no significant change in the results when we increased this number to over 500.

First, we evaluate the label imputation method. Different weight values, $1 \leq w \leq$ 20 , in label imputation are compared in terms of success ratios in diagnosis, as shown in Figure 5.3 and Figure 5.4. We observe that too small or large a choice of $w$ leads to reduced diagnosis accuracy. For example in Board 1, if we consider $50 \%$ missing syndromes, the $\mathrm{SR}$ is only $75 \%$ for $w=1$. As we increase $w$, the $\mathrm{SR}$ increases to $85 \%$ 


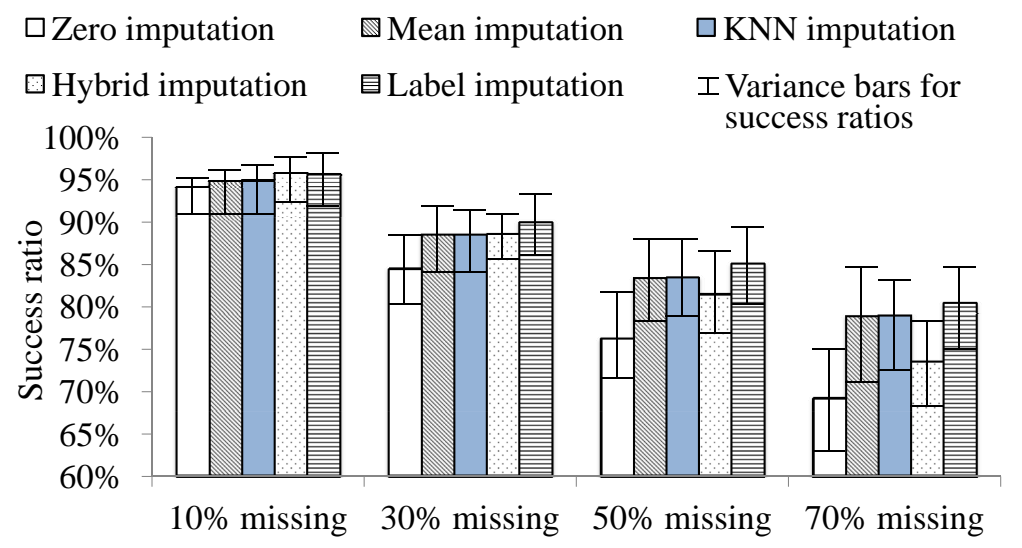

Figure 5.5: Comparison of success ratios for diagnosis using different imputation methods for Board 1.

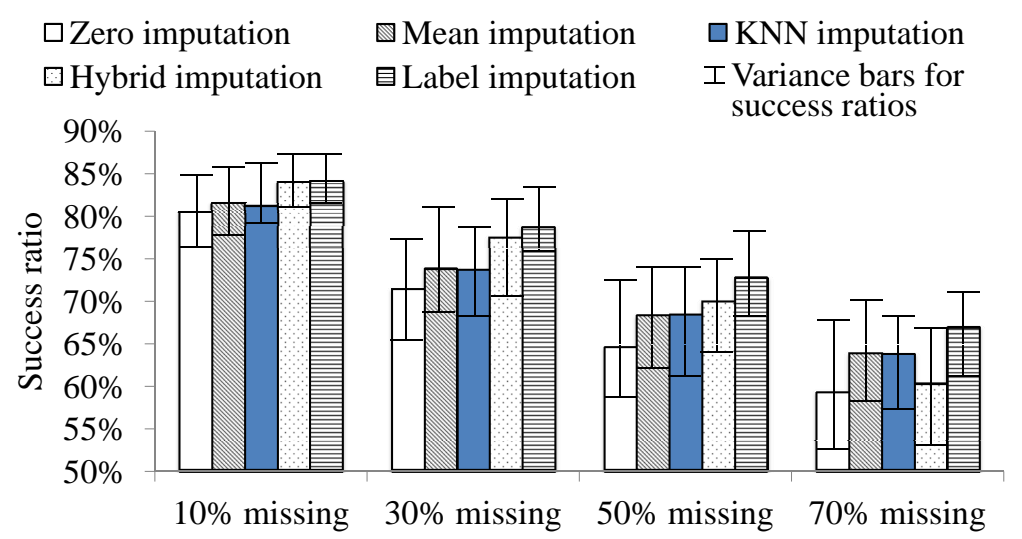

Figure 5.6: Comparison of success ratios for diagnosis using different imputation methods for Board 2.

for $w=4$. However, as we continue to increase $w$, the SR slightly decreases. The SR obtained by using $w=20$ is $82 \%$. Similar SR results are obtain for other missing rates for both Board 1 in Figure 5.3 and Board 2 in Figure 5.4. The reason that smaller $w$ leads to lower SR is that the use of missing syndromes with a small value of $w$ masks the fail syndromes; the fail syndromes are, however, more informative for diagnosis. On the contrary, the reason that too large a value of $w$ leads to lower $\mathrm{SR}$ is that the missing syndromes do not contribute to diagnosis in these cases. If $w$ 


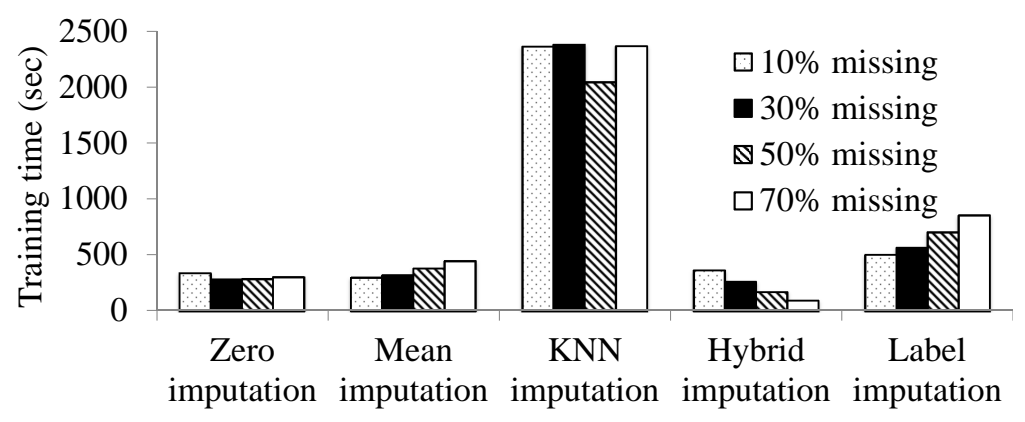

FiguRE 5.7: Comparison of training time for diagnosis system using different imputation methods for Board 1.

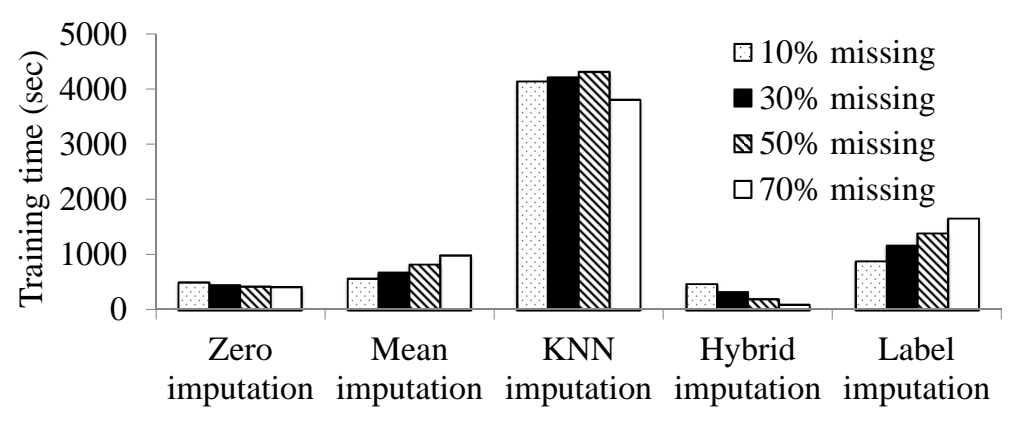

Figure 5.8: Comparison of training time for diagnosis system using different imputation methods for Board 2.

is infinite, the imputation value $d$ becomes 0 for all missing syndromes. In addition, in Figure 5.3 and Figure 5.4, we see that the larger missing rates leads to reduced success ratios, because less accurate diagnosis information can be obtained if fewer syndromes are available to the diagnosis system.

Next, in Figure 5.5 and Figure 5.6, we compare the diagnosis accuracies obtained using different imputation methods for two boards. For example for Board 1 in Figure 5.5, if we consider a 50\% missing rate, the average SR using zero imputation is $76 \%$, the maximum SR is $82 \%$, and the minimum SR is $71 \%$. Note that the zero imputation method is the baseline method corresponding to prior work [40]. The highest average SR is $85 \%$ when we use the label imputation method with $w=4$, 
maximum SR is $89 \%$, and minimum $\mathrm{SR}$ is $79 \%$. Other average $\mathrm{SRs}$ are $82 \%, 82 \%$, and $80 \%$ using mean imputation, KNN imputation, and hybrid imputation, respectively. The label-imputation method leads to higher diagnosis accuracies for other missing rates for Board 1 (Figure 5.5) and Board 2 (Figure 5.6).

Finally, we compare the computational complexity of the different imputation methods for Board 1 in Figure 5.7 and Board 2 in Figure 5.8, respectively, in terms of diagnosis system training time. The training time for the KNN method is much higher than for the other imputation methods. For Board 1, the training time is on average 40 minutes, while the training times for other methods are in the order

of only a few minutes. Therefore, in view of both diagnosis accuracy and training time, the label-imputation method appears to be the best choice for handling missing syndromes.

\subsection{Conclusions}

This chapter has described the design of a smart board-level diagnosis system that can handle missing syndromes using several imputation methods, among which the label-imputation method appears to be the most promising choice. Two synthetic boards generated from two actual board designs have been used to validate the effectiveness of the proposed method. 


\section{6 \\ Information-Theoretic Syndrome and Root-Cause Evaluation}

Reasoning-based methods are promising because a detailed system model is not needed to construct the diagnosis system $[2,23]$. The diagnosis engine is incrementally built based on the database of successfully repaired faulty boards. Machine-learning techniques enable reasoning-based diagnosis, leveraging ease of implementation, high diagnosis accuracy, and continuous learning. Repairs of faulty components are suggested through a ranked list of suspect components, e.g., based on artificial neural networks (ANNs) and support-vector machines (SVMs) [40]. However, a machine learning-based diagnosis system requires a sizeable database for training. Ambiguous root-cause identification may result if the diagnosis system lacks a sufficient database with adequate information for mapping errors to root causes. There is a need for a rigorous framework that can evaluate an existing diagnosis system using quantifiable metrics, and provide means for enhancing the accuracy of diagnosis. Ambiguity in root-cause identification confounds diagnosis, hence such ambiguities need to be identified and used as a guideline for test development. The accuracy of diagnosis and time needed for diagnosis also depend on the quality of syndromes 


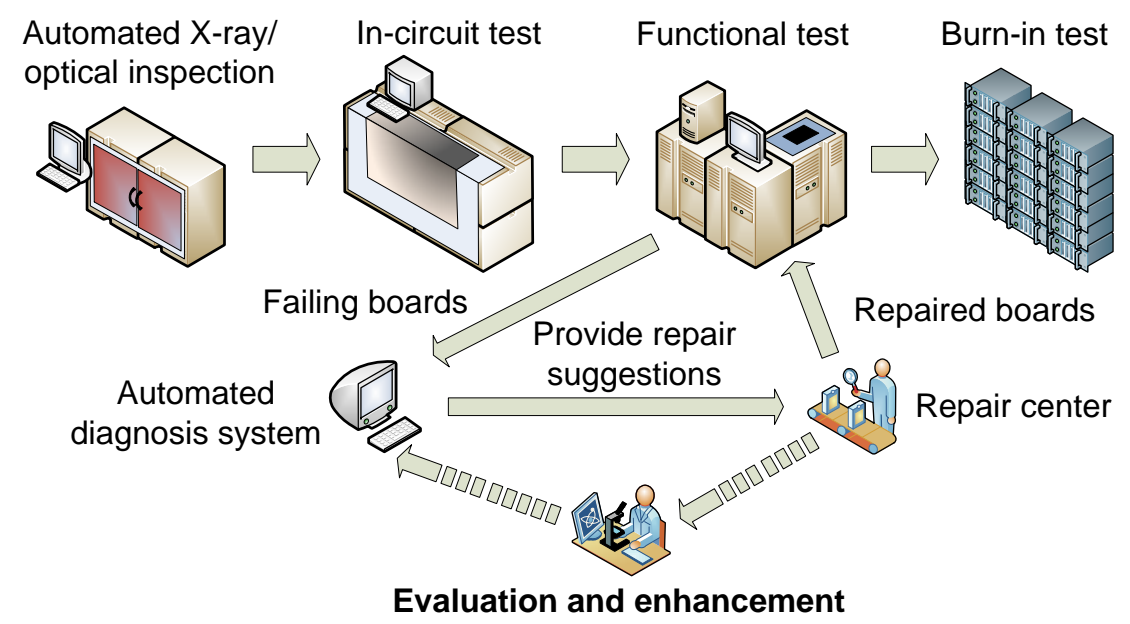

FiguRE 6.1: Overview of the diagnosis system with self-evaluation.

(erroneous observations). Redundant or irrelevant syndromes not only lead to long diagnosis time, but also increase diagnosis complexity.

In this chapter, we propose an evaluation and enhancement framework for a functional fault diagnosis system, as shown in Figure 6.1. This framework is based on information-theoretic analysis. The discriminative ability of each root cause is calculated, which allows us to screen out root causes with low discriminative ability, and identify ambiguous root-cause pairs. The evaluation system also carries out rigorous syndrome analysis. A subset of representative syndromes is selected with maximum relevance and minimum redundancy. The irrelevant or redundant syndromes can subsequently be analyzed to derive better tests for diagnosis.

The remainder of this chapter is organized as follows. Section 6.1 presents the problem and describes the work contributions. Section 6.2 describes the evaluation framework based on subset-selection and class-relevance statistics. Section 6.3 describes the diagnosis framework that includes the proposed evaluation system. Section 6.4 presents results for two industrial boards at volume production. Compared to traditional metrics such as overall success ratios, the proposed evaluation framework provides detailed analysis for each syndrome and each root cause with 


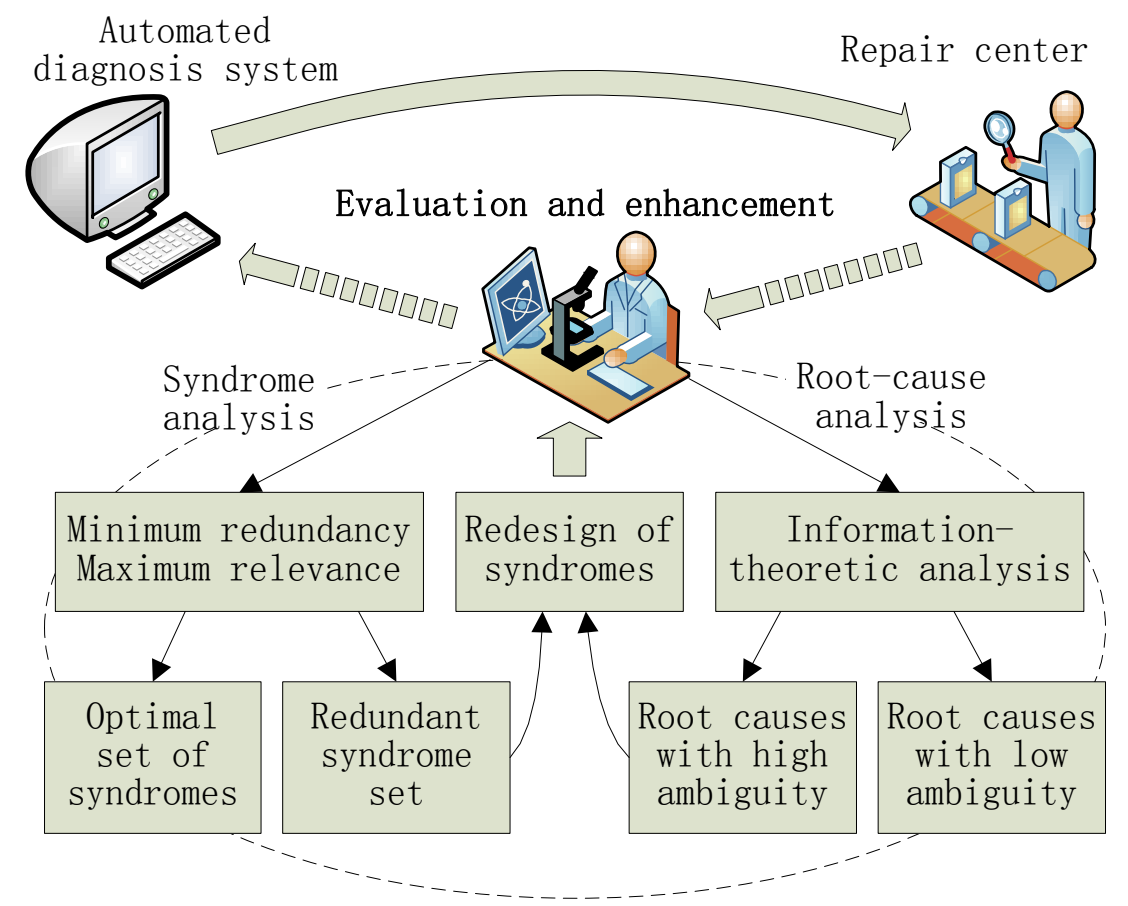

FiguRE 6.2: Evaluation framework for a diagnosis system and the targeted problems.

respect to their contribution to prediction accuracy. Finally, Section 6.5 concludes the chapter.

\subsection{Problem Statement and Chapter Contributions}

Machine learning provides an unprecedent opportunity for developing an automated functional fault diagnosis system that can become more effective over time through self-learning. The use of an automated diagnosis system involves less human effort and can provide accurate diagnostic guidance. The diagnosis system can be built through learning from historical repair data, which determines the prediction performance for future faulty boards. However, the quality of historical data varies across different types of boards and different stages of diagnosis system deployment. In prior work, evaluation of a diagnosis system has been based on success ratio (SR), 
which is defined as the ratio of the number of correctly diagnosed boards to the total number of failed boards. However, SR only offers an overall performance metric for the diagnosis system without providing any guidance for diagnosis system improvement. It is therefore necessary to develop an evaluation framework for the diagnosis system, which can provide valuable feedback for selecting syndromes, designing tests, and reasoning about root-cause ambiguities.

We propose a new evaluation framework, as shown in Figure 6.2, that leverages two analysis methods, targeting syndromes and root-cause components, respectively. Syndrome analysis based on subset selection is used to select an effective, but reduced, set of syndromes for use by a diagnosis system, such as based on SVMs [40]. Root-cause analysis relies on information-theoretic metrics such as precision and recall. These metrics measure the discriminative ability of a diagnosis system to differentiate a root cause from other candidates. The proposed frameworks aims to provide guidelines for addressing the following practical problems:

Lack of experience for particular root causes: It is often the case that a diagnosis system is initially deployed with a limited number of faulty boards and insufficient history data. Boards with a particular root cause may rarely occur in the corresponding history cases. During subsequent diagnosis, if we encounter a board with such a root cause, the diagnosis system will not provide any useful information. The identification of these root causes in an early stage of diagnosis-system deployment will facilitate the evaluation of the system and point out weaknesses.

Ambiguous root causes: The components at board level tend to be coupled to each other, e.g., through layout proximity and signal flow. Given a set of syndromes, a diagnosis system may suggest several potential root causes with the same level of confidence. One reason for such ambiguity is that functional-test packages are typically designed by multiple teams. It can be hard to isolate root causes from each other due to the lack of some key syndromes. It is therefore desirable that the 
evaluation framework reveal these coupled components and provide guidelines for generating new tests.

Redundant or irrelevant syndromes: Although multi-site design collaboration can reduce product release time and boost productivity, syndrome sets from different teams may overlap. Redundant syndromes not only add to diagnosis cost, but also increase diagnosis complexity. A representative set of syndromes can be extracted from the original syndrome set (Figure 6.3), while the remaining redundant syndromes can be used for careful analysis at various levels of design abstraction.

The contributions of the proposed evaluation and enhancement framework are listed below:

- A method to analyze the ability of the diagnosis system to discriminate among root causes.

- A technique to analyze and optimize a syndrome set to obtain a small subset with high discriminative ability.

- Guidelines for test-design teams so that information about redundant syndromes and ambiguous pairs of root causes can be used for future test optimization.

\subsection{Evaluation Methods for Diagnosis Systems}

In this section, we describe the proposed techniques for evaluating a reasoning-based diagnosis system.

\subsubsection{Subset Selection for Syndromes Analysis}

Subset selection (also known as feature selection) [74,75] aims to identify a set of

most important features for characterization. Suppose we have a database $C$ of 


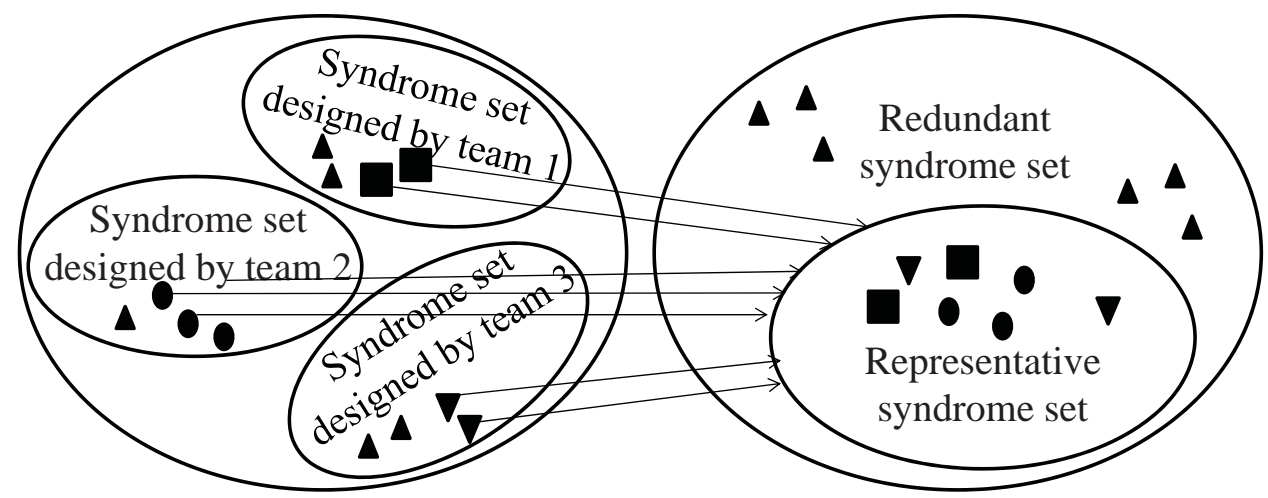

The complete set of syndromes

The complete set of syndromes

FiguRE 6.3: Selection of a representative syndrome subset.

successfully repaired faulty boards, which have $N$ potential root-cause candidates denoted by the elements of the set $\mathbf{A}=\left\{A_{1}, A_{2}, \ldots, A_{N}\right\}$. To diagnose the root cause for a faulty board, we start with $M$ syndromes, i.e., $\mathbf{S}=\left\{S_{1}, S_{2}, \ldots, S_{M}\right\}$. The feature selection problem is to find $m$ syndromes, $m \leq M$, that optimally characterize A (see right part of Figure 6.3). The optimal characterization condition involves the determination of the minimum classification error.

One of the most popular approaches to the subset-selection problem is based on the rule of max-relevance (MaxRel) feature selection. The goal is to select syndromes with the highest relevance to the root cause $A_{i}$. Relevance is characterized in terms of mutual information, which is one of the widely used metrics to define dependency. For each target root cause $A_{i}$ and a given syndrome $S_{j}$, their mutual information $E\left(A_{i}, S_{j}\right)$ is calculated as:

$$
E\left(A_{i}, S_{j}\right)=-p\left(A_{i} \mid s_{j}\right) \log p\left(A_{i} \mid s_{j}\right)-p\left(A_{i} \mid \overline{s_{j}}\right) \log p\left(A_{i} \mid \overline{s_{j}}\right)
$$

where the logarithmic base is $2, s_{j}$ is the event that syndrome $S_{j}$ manifests itself, and $\overline{s_{j}}$ is the complementary event. The measure $p\left(A_{i} \mid s_{j}\right)$ is the probability of $A_{i}$ being the root cause if $S_{i}$ manifests itself. We then calculate the relevance value $D\left(\mathbf{A}, S_{j}\right)$ 
between the syndrome $S_{j}$ and root-cause set $\mathbf{A}$ as follows:

$$
D\left(\mathbf{A}, S_{j}\right)=\frac{1}{|\mathbf{A}|} \sum_{A_{i} \in \mathbf{A}} E\left(A_{i}, S_{j}\right)
$$

The syndromes in $\mathbf{S}$ can be ranked according to $D\left(\mathbf{A}, S_{j}\right)$ values in descending order, where $D\left(\mathbf{A}, S_{1}^{\prime}\right) \geq D\left(\mathbf{A}, S_{2}^{\prime}\right) \geq \cdots \geq D\left(\mathbf{A}, S_{M}^{\prime}\right)$. The MaxRel set $\mathbf{S}^{\prime}=$ $\left\{S_{1}^{\prime}, S_{2}^{\prime}, \ldots, S_{m}^{\prime}\right\}$ is a selected subset of the top $m$ syndromes from $\mathbf{S}$ according to the ranked syndrome list, where $1 \leq m \leq M$ and $\mathbf{S}^{\prime} \subseteq \mathbf{S}[74]$. We can determine the best choice of $m$ by using a heuristic to evaluate the diagnosis system with different syndrome sets $\mathbf{S}^{\prime}$ (each with a different value of $m$ ) in terms of diagnosis accuracy. However, in feature selection, it is recognized that the combinations of individually good features do not necessarily lead to effective classification. In other words, "the $m$ best syndromes are not the best $m$ syndromes" [76,77]. To reduce the redundant syndromes in the syndrome set $\mathbf{S}^{\prime}, \mathbf{S}^{\prime}$ is therefore further evaluated by computing its redundancy value $R\left(\mathbf{S}^{\prime}\right)$ as shown below:

$$
R\left(\mathbf{S}^{\prime}\right)=\frac{1}{\left|\mathbf{S}^{\prime}\right|^{2}} \sum_{S_{i}^{\prime} \in \mathbf{S}^{\prime}} \sum_{S_{j}^{\prime} \in \mathbf{S}^{\prime}} E\left(S_{i}^{\prime}, S_{j}^{\prime}\right),
$$

where $E\left(S_{i}^{\prime}, S_{j}^{\prime}\right), S_{i}^{\prime} \neq S_{j}^{\prime}$, is the mutual information between $S_{i}^{\prime}$ and $S_{j}^{\prime}$, which is similar to the definition of $E\left(A_{i}, S_{j}\right)$, i.e., $E\left(S_{i}^{\prime}, S_{j}^{\prime}\right)=-p\left(s_{i} \mid s_{j}\right) \log p\left(s_{i} \mid s_{j}\right)-$ $p\left(s_{i} \mid \bar{s}_{j}\right) \log p\left(s_{i} \mid \bar{s}_{j}\right)-p\left(\bar{s}_{i} \mid s_{j}\right) \log p\left(\bar{s}_{i} \mid s_{j}\right)-p\left(\bar{s}_{i} \mid \bar{s}_{j}\right) \log p\left(\bar{s}_{i} \mid \bar{s}_{j}\right)$. We then calculate the minimum-redundancy maximum-relevance $(m R M R)$ value for each $\mathbf{S}^{\prime}$, as follows:

$$
m R M R\left(\mathbf{S}^{\prime}\right)=\frac{1}{\left|\mathbf{S}^{\prime}\right|} \sum_{S_{k}^{\prime} \in \mathbf{S}^{\prime}} D\left(\mathbf{A}, S_{k}^{\prime}\right)-R\left(\mathbf{S}^{\prime}\right)
$$

We can next determine the minimum-redundancy-maximum-relevance (mRMR) syndrome subset $\mathbf{S}^{*}$ with the largest $m R M R$ value, as follows:

$$
\mathbf{S}^{*}=\max _{\mathbf{S}^{\prime}}\left\{m R M R\left(\mathbf{S}^{\prime}\right)\right\}
$$


Table 6.1: Confusion matrix.

\begin{tabular}{|c|c|c|c|}
\hline \multicolumn{2}{|c|}{} & \multicolumn{2}{c|}{ Predicted class } \\
\cline { 3 - 4 } \multicolumn{2}{|c|}{} & Positive & Negative \\
\hline \multirow{2}{*}{$\begin{array}{c}\text { Actual } \\
\text { class }\end{array}$} & Positive & TP & FN \\
\cline { 2 - 4 } & Negative & FP & TN \\
\hline
\end{tabular}

We can now evaluate the diagnosis system with the mRMR syndrome set $\mathbf{S}^{*}$ in terms of diagnosis accuracy. The subset of syndromes thus selected leads to minimum classification error.

\subsubsection{Class-Relevance Statistics}

Success ratio (SR) has been used for evaluating board-level diagnosis systems in prior work [40]. However, SR is a coarse metric for multi-class classifiers (e.g., a board-level functional fault diagnosis system) and does not provide any suggestion for improving diagnosis. We introduce a fine-grained set of information-theoretic metrics, which are used for comprehensively evaluating the diagnosis system. First, we use the concept of a confusion matrix, a table layout that allows visualization of the performance of a classifier. Each column of the matrix represents the cases in a predicted root cause, and each row represents the cases of an actual root cause. The name stems from the fact that it makes it easy to see if the system is confusing between root causes. For example in Table 6.1, we form a representative binary classification system with two classes encoded as positive and negative, respectively. The entries in the confusion matrix have the following meaning:

- $\mathrm{TP}$ is the number of correctly predicted positive cases;

- FP is the number of incorrectly predicted positive cases;

- FN is the number of incorrectly predicted negative cases;

- $\mathrm{TN}$ is the number of correctly predicted negative cases. 
Two popular metrics (precision and recall) make up the proposed root-cause analysis framework.

Positive predictive value (PPV), also known as precision, is the proportion of the predicted positive cases that are correct, calculated using the equation [77]:

$$
\mathrm{PPV}(\text { precision })=\frac{\mathrm{TP}}{\mathrm{TP}+\mathrm{FP}}
$$

True positive rate (TPR), also known as recall, is the proportion of positive cases that are correctly identified, calculated as follows:

$$
\mathrm{TPR}(\text { recall })=\frac{\mathrm{TP}}{\mathrm{TP}+\mathrm{FN}}
$$

Note that the SR metric can be expressed in terms of recall. It is the sum of all the correctly predicted cases for each root cause $A_{i} \in \mathbf{A}$, calculated as $\mathrm{SR}=$ $\sum_{A_{i} \in \mathbf{A}} \mathrm{TPR}_{A_{i}} \cdot n_{A_{i}}$

A precision score of 1.0 for a root cause $A_{i}$ means that all predictions about positive cases are correct, i.e., there are no false positives. On the other hand, a recall score of 1.0 means that all the cases with root cause $A_{i}$ are correctly predicted, i.e., there are no false negatives. For example, in board-level diagnosis, precision describes the percentage of success in predicting a root cause, while recall reflects the percentage of success for a root cause to be predicted. One metric alone provides only partial information, limited to either prediction or reality. A combination of these two metrics provides a more complete picture. Hence, compared to the traditional metric (SR), we now have a more comprehensive set of metrics, through which we can obtain more information on prediction accuracy. In addition, we also log all ambiguous pairs of root causes, which can be referred to design teams for detailed analysis at various levels of design. 


\subsection{Evaluation and Enhancement Framework}

Prediction accuracy improves when the volume of successfully repaired faulty boards increases [40]. However, in practice, the database is limited in size and the diagnosis system suffers from low prediction accuracy due to the lack of availability learning data. The diagnosis system must therefore be assessed periodically during the deployment to measure its prediction quality.

\section{Evaluation and Enhancement Procedure}

Given a database of failed boards, syndrome and root-cause analyses are processed in parallel in the evaluation framework (see Figure 6.2). The syndrome analysis is based on the minimum redundancy and maximum relevance (mRMR) method. Mutual information between each syndrome and root cause is iteratively calculated to form a set of syndromes with maximum relevance, followed by syndrome reduction from this set based on the calculation of relevance between syndromes. The set of redundant syndromes can then be provided to the syndrome design team for further analysis based on similarity among functional tests. If some of the redundant syndromes are still considered to be useful by the design team, we regard them as "suspicious" syndromes. The redundant syndromes are removed from the new syndrome set for diagnosis, but the "suspicious" ones will be retained in the diagnosis system for future evaluation.

As discussed in Section 6.2, root-cause analysis is based on information-theoretic metrics. First, an $N \times N$ confusion matrix is generated, where $N$ is the number of root causes. For each root cause $A_{i}$, we define a hypothetical root cause $A_{i}^{*}=\mathbf{A}-\left\{A_{i}\right\}$. We then derive a two-class confusion matrix of $A_{i}$ and $A_{i}^{*}$ from the original confusion matrix. If the precision of $A_{i}$ is less than one, for each faulty board, we create ambiguous root cause pair $\left\langle A_{i}, A_{j}\right\rangle$, where $A_{j}$ is the root cause for a board incorrectly 
predicted to be $A_{i}$. On the other hand, if the recall of $A_{i}$ is less than one, for each faulty board, we form the root cause pair $\left\langle A_{k}, A_{i}\right\rangle$, where $A_{k}$ is the incorrect root cause prediction for a board with the root cause $A_{i}$. These ambiguous pairs and associated faulty boards will then be provided to the syndrome design team. New tests (resulting in new syndromes) can be developed to split these ambiguous pairs and they can be added into the new syndrome set to improve the diagnosis system. The outputs of this evaluation and enhancement framework include:

- An optimal set of syndromes that contribute to root cause isolation;

- A set of redundant or irrelevant syndromes to be discarded based on the outcome of functional analysis;

- A group of root causes that can be isolated with high confidence;

- A set of ambiguous root-cause pairs, and associated boards for detailed functional analysis.

Based on the outputs of the proposed evaluation framework, new syndromes can be designed to increase the discriminative ability for ambiguous root-cause pairs, and redundant or irrelevant syndromes can be discarded to reduce diagnosis complexity. Based on the new syndrome set, a new diagnosis system can be trained using methods such as fault-insertion tests, FPGA-based board test, model-based reasoning, etc. $[27,43]$.

An Example of The Proposed Framework

Consider a hypothetical demonstration board with five cases that are used for evaluation. Let $x_{1}, x_{2}, x_{3}$, and $x_{4}$ be four syndromes. If the syndrome manifests itself, we record it as 1, and 0 otherwise. Let us suppose that the board has two candidate root causes $\mathrm{A}$ and $\mathrm{B}$, and we encode them as $y=P$ and $y=N$, respectively. In a 
real scenario, fault syndromes vary across products and tests. Here, we merge the syndromes and the known root causes in one matrix $\mathcal{A}=(\mathcal{B} \mid \mathcal{C})$, where the left $(\mathcal{B})$ side refers to the syndromes, while the right side $(\mathcal{C})$ refers to the corresponding fault classes. This matrix represents the database information for evaluation.

$$
\mathcal{A}=[\mathcal{B} \mid \mathcal{C}]=\left[\begin{array}{cccc:c}
1 & 1 & 0 & 0 & P \\
1 & 1 & 0 & 1 & P \\
1 & 1 & 0 & 0 & P \\
0 & 1 & 1 & 1 & N \\
0 & 0 & 1 & 1 & N
\end{array}\right]
$$

First, we consider syndrome analysis based on the database. The two-step mRMR method consists of calculating relevance scores and mRMR scores. The mutual information for root cause $P$ are calculated to be 0 for $x_{1}, 0$ for $x_{3},-0.27$ for $x_{2}$ and -0.38 for $x_{4}$ in a descending order, using Equation (6.2). We can select a subset of one syndrome $\left\{x_{1}\right\}$ with the relevance score of 0 . If we select a subset of two syndromes, we can select $\left\{x_{1}, x_{2}\right\}$ with the relevance score of $(0-0.27) / 2=-0.13$, or $\mathbf{S}^{\prime}=\left\{x_{1}, x_{3}\right\}$ with the relevance score of $(0+0) / 2=0$ and so on. However, we observe that $x_{3}$ is the complement of $x_{1}$. To minimize redundancy in the subset, we then calculate the redundancy score to get the mRMR score using Equation (6.4). The redundancy score $R\left(\mathbf{S}^{\prime}\right)=\frac{1}{4} E\left(x_{1}, x_{3}\right)=0$, thereby resulting in the $m R M R\left(\mathbf{S}^{\prime}\right)=0-0=0$ for $\mathbf{S}^{\prime}$. Therefore, $x_{3}$ is considered to be redundant if $\left\{x_{1}\right\}$ can be used for classification. Consider two other two-class subsets of syndromes, namely $\left\{x_{1}, x_{2}\right\}$ with $m R M R=-0.07$, and $\left\{x_{1}, x_{4}\right\}$ with $m R M R=-0.10$. Based on these numbers, we still choose a one-syndrome subset $\left\{x_{1}\right\}$ with higher $m R M R$ score of 0 for diagnosis. To ensure that $\left\{x_{1}\right\}$ is the minimal syndrome subset, we also use SR to estimate the performance of the diagnosis system using this syndrome subset. We observe that the SRs obtained from a diagnosis system using other multisyndrome sets are no better than using $\left\{x_{1}\right\}$. We then select the optimal syndrome set $\mathbf{S}^{*}=\left\{x_{1}\right\}$ for this diagnosis system. 
Next, we consider root-cause analysis. Suppose the prediction of a diagnosis system for these five boards is $\{P, P, N, N, N\}$, wherein the third board with root cause $P$ is predicted to be $N$, which is incorrect. In the confusion matrix, $\mathrm{TP}=2$, $\mathrm{FP}=1, \mathrm{FN}=0$, and $\mathrm{TN}=2$. Thus, precision of $P$ is calculated to be 0.66 , which means two out of three predictions of root cause $P$ are correct. In addition, recall of $P$ is 1 , which mean all the boards with root cause $P$ are correctly predicted.

\subsection{Experiments and Results}

We conducted the experiments on three industrial boards that are currently in highvolume production. Here, we describe the experimental setup and then present the improvements achieved by using the evaluation framework.

\subsubsection{Experimental setup}

Both syndrome and root-cause analysis are implemented in $\mathrm{C} / \mathrm{C}++$. Syndrome analysis is implemented using minimum-redundancy-maximum-relevance toolkits [74]. Note that the proposed evaluation framework is of general use to any diagnosis system. We illustrate the use of the evaluation framework using two examples, i.e., a support-vector machine (SVM)-based diagnosis system and an artificial neuralnetwork (ANN)-based diagnosis system, respectively [40].

We use two industrial boards from Table 2.1 to illustrate the evaluation framework. Information about these two industrial boards, diagnosis results before evaluation, and diagnosis results after evaluation and test redesign are reproduced and shown in Table 6.2 for an SVM-based diagnosis system and Table 6.3 for an ANNbased diagnosis system. For example, a total of 1613 repaired boards are collected before evaluation from the contract manufacturers database for Board 1. A total of 546 fault syndromes are extracted from the failure logs. The number of faulty components identified in the database for repair action is 153 . After we apply the 
Table 6.2: Diagnosis results before and after evaluation for a diagnosis system based on SVMs.

\begin{tabular}{|c|c|c|}
\hline & Board 1 & Board 2 \\
\hline Number of syndromes & 546 & 375 \\
Number of components & 153 & 116 \\
Number of boards & 1613 & 1351 \\
$\mathrm{SR}_{1}$ & $73.2 \%$ & $66.3 \%$ \\
$\mathrm{SR}_{2}$ & $80.4 \%$ & $74.3 \%$ \\
$\mathrm{SR}_{3}$ & $88.2 \%$ & $84.1 \%$ \\
\hline \multicolumn{2}{|c|}{ Post-evaluation } \\
\hline Number of syndromes & 299 & 201 \\
Number of components & 153 & 116 \\
Number of boards & 256 & 224 \\
$\mathrm{SR}_{1}$ & $86.1 \%$ & $89.6 \%$ \\
$\mathrm{SR}_{2}$ & $90.8 \%$ & $90.9 \%$ \\
$\mathrm{SR}_{3}$ & $93.2 \%$ & $92.0 \%$ \\
\hline
\end{tabular}

evaluation framework on Board 1, additional 256 boards are evaluated.

To ensure real-time diagnosis and repair, we assume that we are allowed at most three attempts to replace the potential failing components. We define $\mathrm{SR}_{1}$ as the success ratio corresponding to the case that the board is deemed to have been successfully repaired only when the actual faulty component is identified and placed at the top of the list of root-cause candidates. We also define $\mathrm{SR}_{2}\left(\mathrm{SR}_{3}\right)$ as the success ratio corresponding to the case that a board is deemed to have been successfully repaired if the actual faulty component is in the first two (three) positions in the list of root-cause candidates.

\subsubsection{Results}

We first do the syndrome analysis, followed by root-cause analysis. To evaluate the effectiveness and efficiency of syndrome analysis, we examine the minimum number of syndromes used by the diagnosis system, while achieving the same diagnosis accuracy 
Table 6.3: Diagnosis results before and after evaluation for a diagnosis system based on ANNs.

\begin{tabular}{|c|c|c|}
\hline & Board 1 & Board 2 \\
\hline Number of syndromes & 546 & 375 \\
Number of components & 153 & 116 \\
Number of boards & 1613 & 1351 \\
$\mathrm{SR}_{1}$ & $67.9 \%$ & $57.9 \%$ \\
$\mathrm{SR}_{2}$ & $78.1 \%$ & $70.3 \%$ \\
$\mathrm{SR}_{3}$ & $85.4 \%$ & $75.1 \%$ \\
\hline \multicolumn{2}{|c|}{ Post-evaluation } \\
\hline Number of syndromes & 275 & 202 \\
Number of components & 153 & 116 \\
Number of boards & 256 & 224 \\
$\mathrm{SR}_{1}$ & $81.2 \%$ & $76.4 \%$ \\
$\mathrm{SR}_{2}$ & $86.5 \%$ & $87.5 \%$ \\
$\mathrm{SR}_{3}$ & $93.2 \%$ & $89.3 \%$ \\
\hline
\end{tabular}

obtained with the entire set of syndromes. Table 6.2 and Table 6.3 shows the number of syndromes before and after the syndrome analysis for the three boards for an SVMbased and an ANN-based diagnosis systems, respectively. For example, for Board 1 using SVM, the number of syndromes after analysis is reduced to 299, which is only $54 \%$ of the total number of syndromes before syndrome analysis.

There are several sources of redundancy that contribute to the significant reduction in the number of syndromes:

1. A few syndromes are effective indicators of failing boards but they are insensitive to the specific root cause that results in the board failure. These syndromes are useful for failure screening, but of no help for fault isolation.

2. A number of syndromes have never manifest themselves in any failing scenario thus far. We regard them as "suspicious" syndromes, but there is a large chance that those syndromes will never be manifested in the future. 


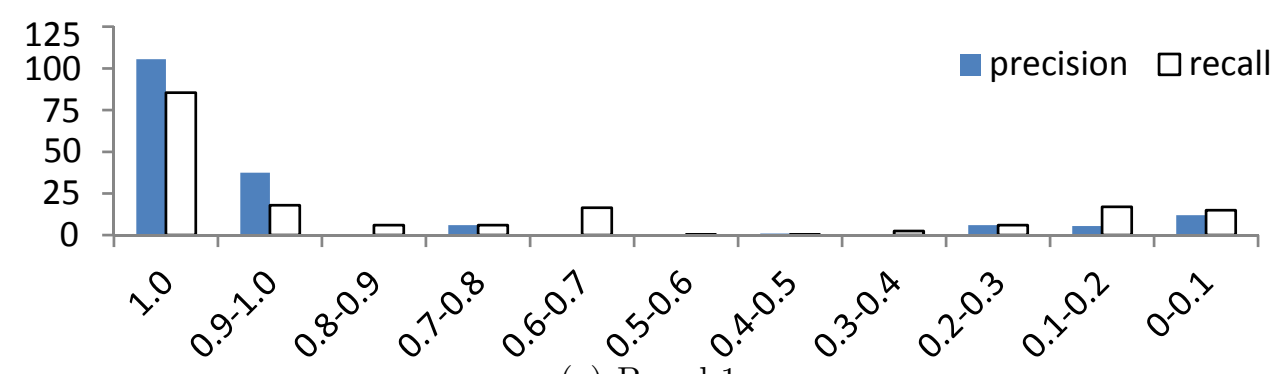

(a) Board 1

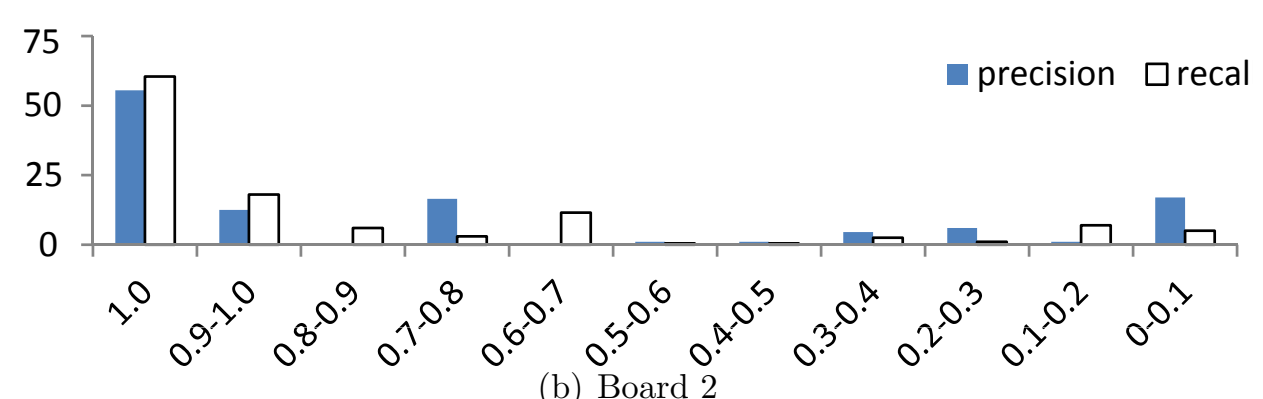

Figure 6.4: Precision and recall distribution for an SVM-based diagnosis system for three boards.

3. Most syndromes are fine-grained at the pin level of the component. However, those coarse-grained syndromes are adequate for fault isolation at component level. We can combine several syndromes for the same component into one syndrome.

To evaluate the effectiveness of root-cause analysis, we present the precision and recall results for the three boards in Figure 6.4-6.5. Most root causes in all boards can be clearly differentiated from others. For example in Board 1 using the SVMbased diagnosis system in Figure 6.4(a), the number of root causes with precision of 1.0 is 105 , while the number of root causes with recall of 1.0 is 83 . In contrast, some root causes cannot be differentiated at all, since either their precision or recall is less than 0.1 . There are a total of 22 root causes of low discriminative ability, of which 13 root causes have both low precision and low recall, 8 root causes only have low precision, and rest 7 root causes only have low recall. The values of precision and recall for Board 2 can be seen in Figure 6.4(b). 


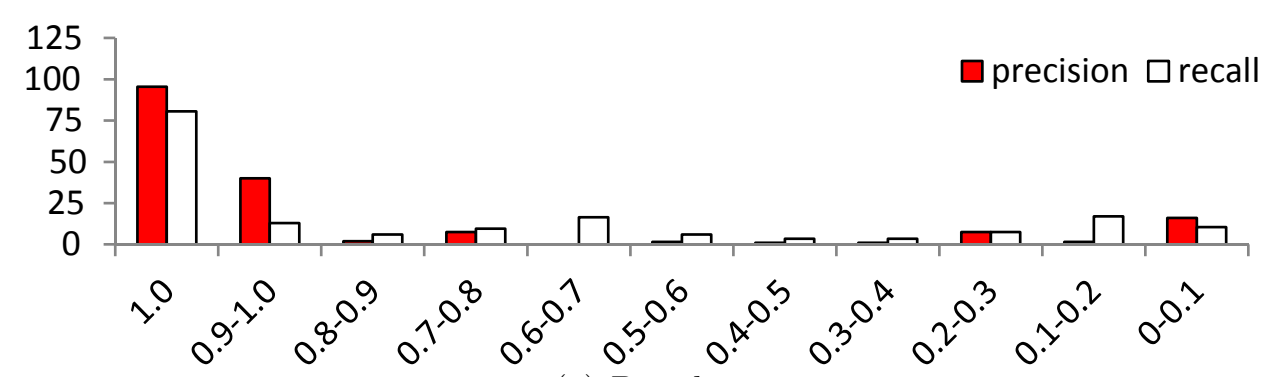

(a) Board 1

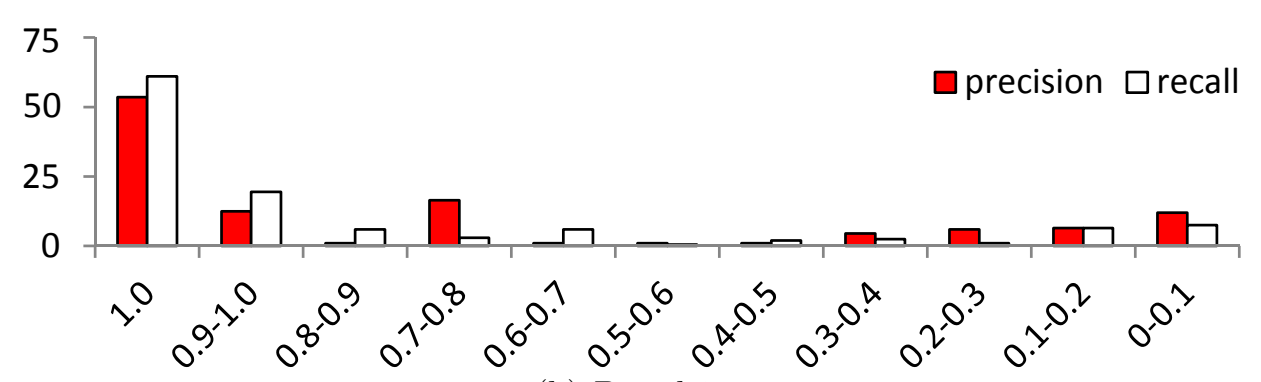

(b) Board 2

Figure 6.5: Precision and recall distribution for an ANN-based diagnosis system for three boards.

Next, we present additional information about ambiguous root-cause pairs from the faulty boards in Figure 6.6-6.7. For example for Board 1 and using SVM, a total of 60031 root-cause pairs are formed for 347 root causes. Out of these, $98.5 \%$ of the pairs can be differentiated, while only $1.5 \%$ of the pairs are ambiguous. We found $0.6 \%$ of the pairs are ambiguous between two root causes, $0.1 \%$ of the pairs in a group of three, $0.8 \%$ of the pairs in a group of four, and $0.1 \%$ of the pairs in a group of eight.

There are several reasons for these ambiguous root-cause pairs:

1. Memories are paired and occupy one address/data path connected to the chip. Limited observability is a consequence of such a design. In this scenario, external memory BIST can help differentiate between those memories.

2. A number of passive components, oscillators, and frequency dividers form a clock chain/tree, where only limited design-for-tests (DfT) features can be in- 


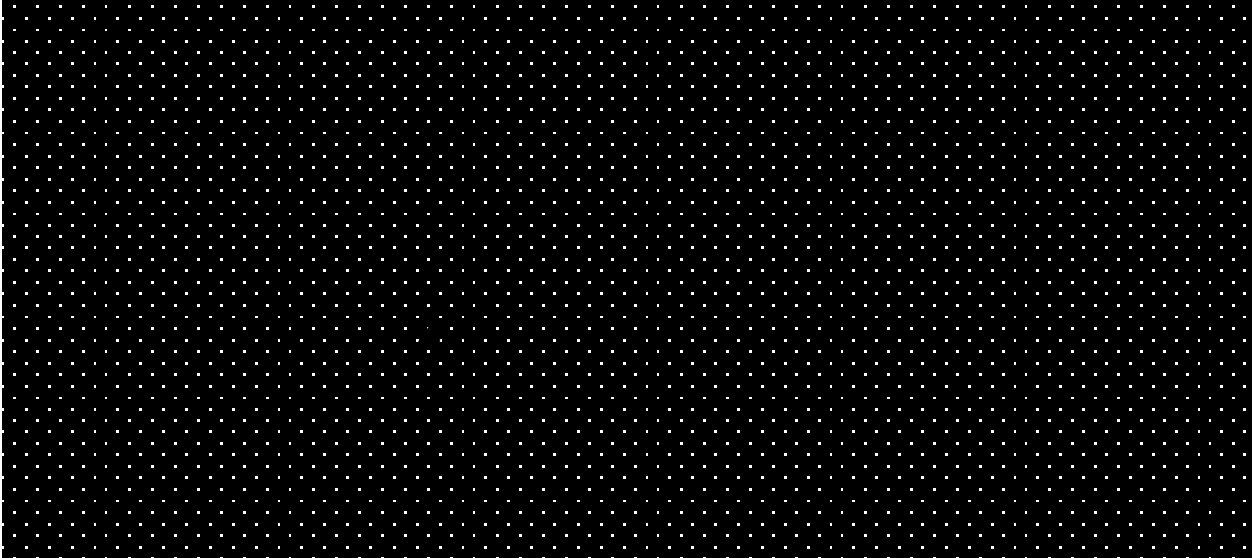

(a) Board 1

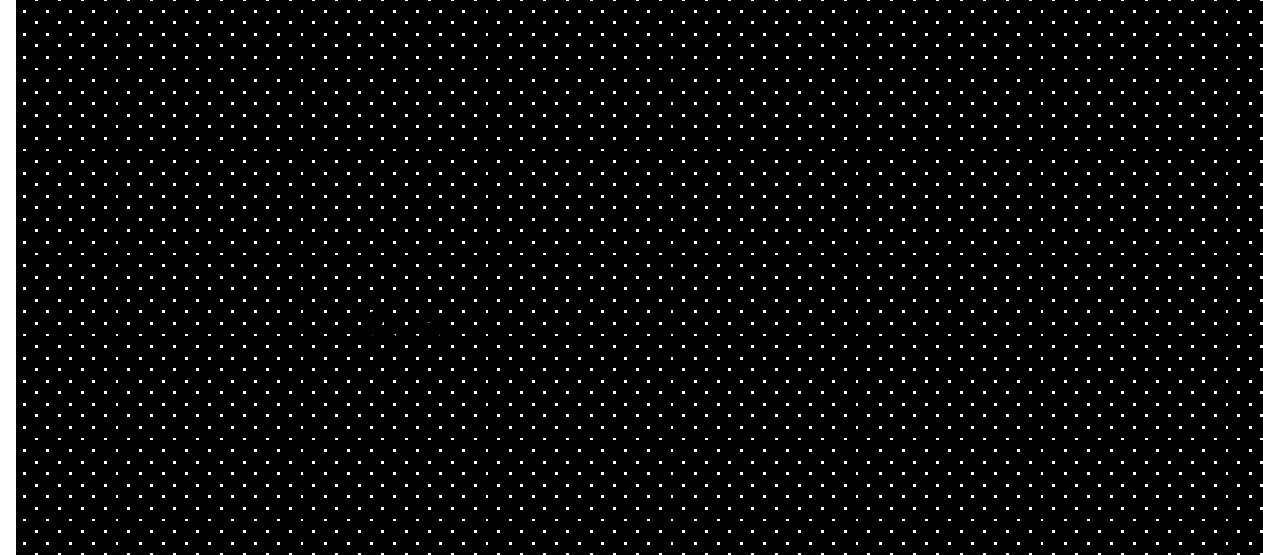

(b) Board 2
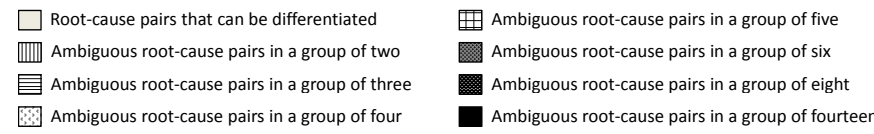

FigurE 6.6: Comparison of ambiguous root-cause pairs for an SVM-based diagnosis system for three boards.

serted. Such DfT limitations can also be observed in the power-supply tree. In such scenarios, increasing the number of observation points on the board is desirable.

3. At the system boot-up stage, the same syndrome is observed due to any candidate defect from a pool of a number of chips and peripheral clock/voltage components, and passive components. In such a scenario, collecting additional 


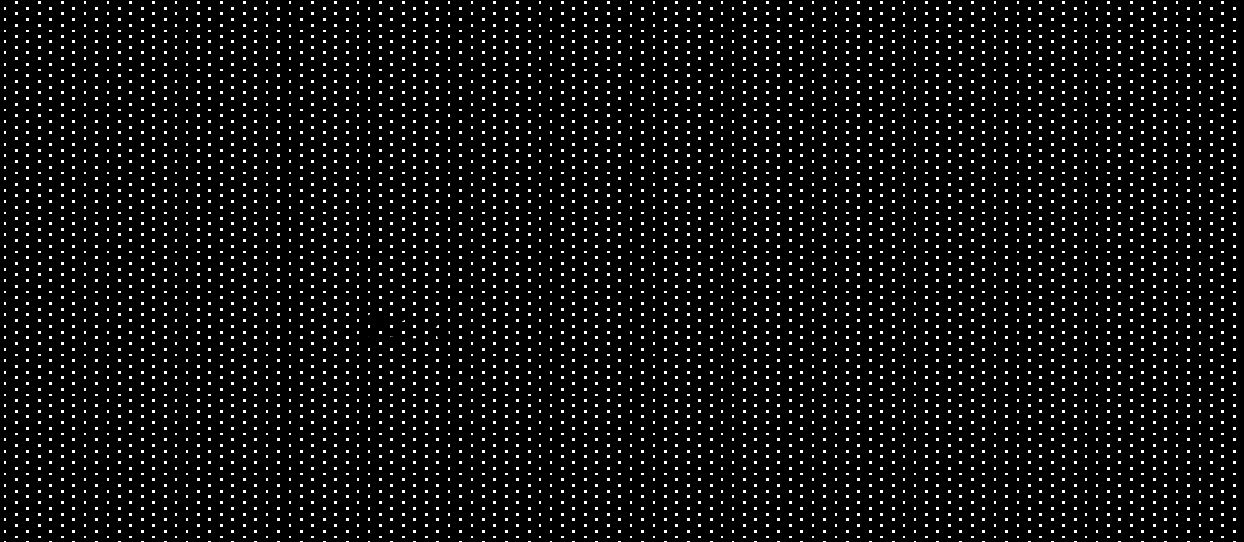

(a) Board 1

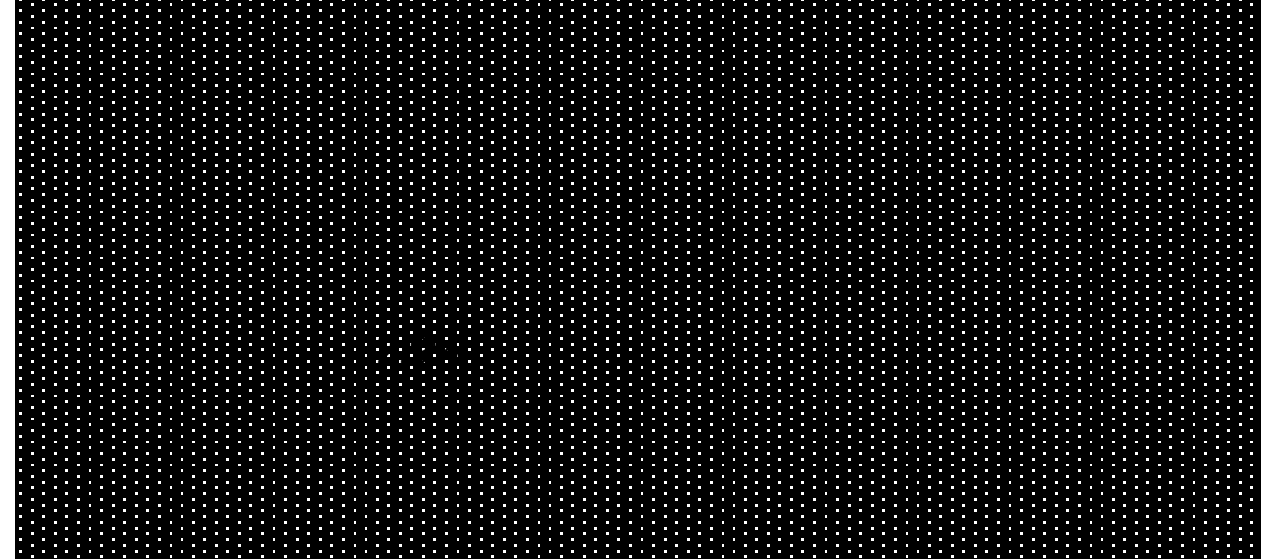

(b) Board 2

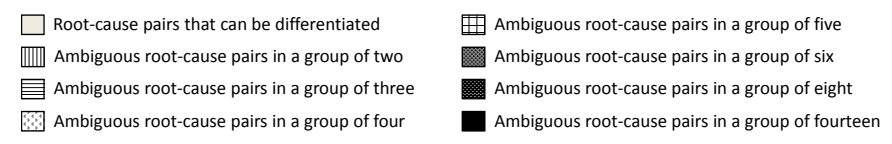

FiguRE 6.7: Comparison of ambiguous root-cause pairs for an ANN-based diagnosis system for three boards.

syndromes at the boot-up stage helps increase discriminative ability between root causes.

Last, we demonstrate the effectiveness of the evaluation framework by evaluating the SRs obtained from the updated set of tests, which are guided by the evaluation framework. As shown in Table 6.2 for SVM-based diagnosis systems and Table 6.3 for ANN-based diagnosis systems, all the success ratios increase. For example for 
SVM-based diagnosis system for Board 1, SR1, SR2, and SR3 are 73.2\%, 80.4\%, and $88.2 \%$, respectively, on a total of 1613 failing boards before the evaluation framework is applied on the diagnosis system. The success ratios increase to $86.1 \%, 92.8 \%$, and $93.2 \%$ on the new 256 failing boards after we increase the discriminative ability of the diagnosis system by adding new tests. The improvement of diagnosis accuracy can also been seen for ANN-based diagnosis systems.

\subsection{Conclusions}

This chapter has proposed an evaluation and enhancement framework based on information-theoretic metrics. Reasoning-based board-level fault diagnosis system requires a rich set of syndromes and a sizable database of faulty boards. However, an insufficient database of faulty boards and redundant syndromes may lead to ambiguous diagnosis, thereby resulting in an increase in diagnosis cost. Syndrome analysis based on subset selection can generate an optimal set of syndromes, and root-cause analysis based on class-relevance metrics can help identify root causes of low discriminative ability. Identifying these redundant syndromes and ambiguous root-cause pairs can provide guidelines for optimizing the syndrome set for diagnosis. Promising experimental results have been obtained from two industry boards in high-volume production. 


\section{7}

\section{Knowledge Discovery and Knowledge Transfer}

Reasoning-based methods have recently become popular since they overcome the knowledge-acquisition bottleneck during volume production and they can automatically generate an intelligent diagnostic system from existing resources $[23,78]$. However, knowledge acquisition is a major problem for a reasoning-based method at the initial product ramp-up stage. Machine learning-based reasoning requires an adequate database for training the reasoning engine, and such a database becomes available much later in the product cycle.

In this chapter, we propose a knowledge-discovery method and a knowledgetransfer method for facilitating board-level functional fault diagnosis at the initial product ramp-up stage. The proposed methods help address the knowledge gap between test-design stage and volume production. First, an analysis technique based on topic model is used to discover knowledge from syndromes, which can be used for training a diagnosis engine. Second, knowledge from diagnosis engines used for earlier-generation products can be automatically transferred through root-cause mapping and syndrome mapping based on keywords and board-structure similarities.

The remainder of this chapter is organized as follows. Section 7.1 describes the 


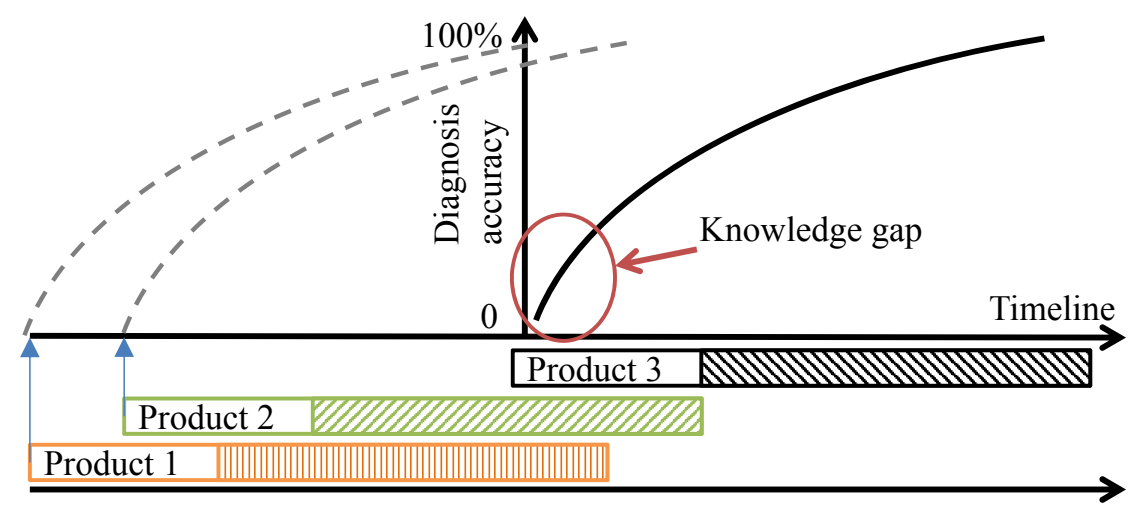

FigURE 7.1: The problem of low accuracy for a reasoning-based diagnosis system at the beginning of a new product (Product 3) ramp-up stage.

"data acquisition" problem in detail and highlights the contribution of this work. Section 7.2 presents an overview of the knowledge discovery and transfer framework in reasoning-based fault diagnosis. Details of the knowledge-discovery solution are described in Section 7.3 and the knowledge-transfer mechanism is described in Section 7.4. Results on knowledge discovery and transfer involving five boards from industry are presented in Section 7.5. The high diagnosis accuracy obtained using the new diagnosis engine demonstrates the effectiveness of the proposed knowledge discovery and transfer framework. Finally, Section 7.6 concludes the chapter.

\subsection{Problem Statement and Chapter Contributions}

Reasoning-based diagnostic systems based on machine learning can automatically derive and exploit knowledge from repair logs of previously documented cases, without requiring detailed understanding of the complex functionality of boards $[40,41]$. The diagnosis accuracy improves when more successfully repaired boards during volume production are available for training the diagnosis system by using incremental learning $[39,59]$. However, there exists a significant knowledge gap in the initial product ramp-up stage, even though knowledge accumulation is badly needed for 
diagnosis system training, as shown in Figure 7.1. Knowledge acquisition is the major bottleneck for developing a usable diagnosis engine. In contrast, rule-based and model-based techniques $[20,27]$ can assist in the initial creation of the knowledge base, but the acquisition of such knowledge requires a technician's expertise and the knowledge may be biased or flawed if the board structure is not fully understood. Model-based reasoning [79] is a hybrid model/reasoning-based approach to bridge the knowledge gap between initial ramp-up and volume production. However this approach is deployed mainly in the form of a model-based method, and reasoning is used only in a limited way, thus considerable human effort is required to maintain the diagnosis engine. The knowledge gap results in ambiguous root-cause prediction, which in turn leads to longer diagnosis time, low yield, and high manufacturing cost.

To view this problem in a more common and realistic scenario, a successful product typically experiences multiple updates and there are often similar products during a period of time; see Figure 7.1. From the hardware point of view, each version update involves incremental changes to the board structure, and the corresponding functional test program needs minor changes as well. The test programs are continually updated until the product becomes mature. However, even if the components on a board and the test programs change incrementally, traditional diagnosis engine has to be trained using the cases diagnosed with the new test programs. Due to the low volume in the ramp-up stage, the number of failing boards for training the new diagnosis engine is very small at this stage. Therefore, it is infeasible to deploy learning-based diagnosis engine in the early manufacturing phase of a new product. Hence, there is a major incentive to explore new methods of knowledge acquisition. We propose a knowledge-discovery and knowledge-transfer framework as a solution to the problem of knowledge acquisition for the newer-generation diagnosis engine.

The contributions of this work can therefore be detailed as follows: 


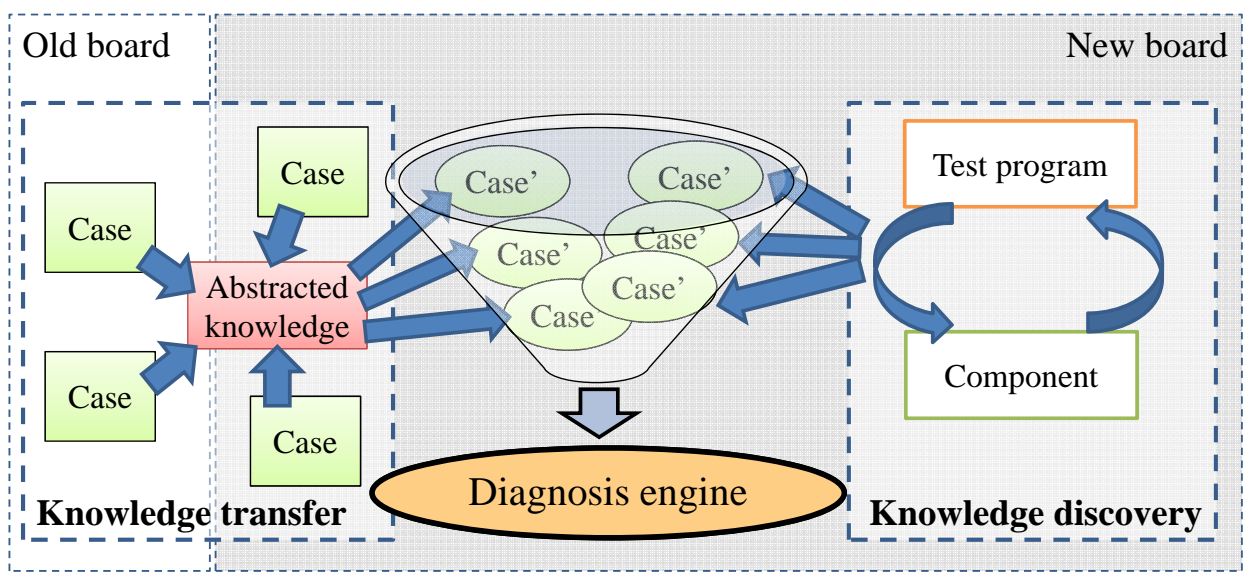

FiguRE 7.2: Illustration of knowledge-discovery and transfer framework for boardlevel diagnosis.

1. Automated knowledge acquisition and filling of the knowledge gap during the product ramp-up stage.

2. Bridging the model-based diagnosis engine with reasoning-based diagnosis to overcome the knowledge acquisition bottleneck at the ramp-up stage.

3. Automated extraction of the relationship between root-causes and syndromes through the concept of topic model.

4. Knowledge discovery and reuse of prior knowledge from the previous diagnosis system and its use in the new diagnosis engine, thus enabling knowledge inheritance among products.

\subsection{Overview of Knowledge Discovery and Transfer Framework}

The proposed knowledge-discovery and knowledge-transfer framework enhances the capability of a reasoning-based diagnosis engine, by providing knowledge from insourcing and outsourcing in the form of synthetic cases using the proposed knowledge discovery and knowledge transfer methods, respectively, as shown in Figure 7.2. 
Insourcing relies on knowledge discovery, which extracts knowledge, reflected as the relationship between root-causes and test programs, from the test logs. In prior work [40], syndromes extracted from the test log and the recorded root-causes are fed to the diagnosis engine for learning. Defining and extracting syndromes from logs is a critical step in the development of an automatic diagnosis system. However, this step relies on the experience of test engineers and hence it consumes a significant amount of time. Once syndromes are defined, they are simply features for the classification engine. Not much attention has been paid in the past to the content of these syndromes and the automation of syndrome extraction, even though syndromes include valuable information for root-cause isolation. The knowledgediscovery approach can facilitate engineers to mine useful knowledge from the log, reflected as specific keywords, because the test program is designed intentionally to contain as much (unstructured) information as possible to help isolate root-causes. The proposed knowledge-discovery approach can be regarded as an example of a "topic model", which is discussed in detail in Section 7.3.

Outsourcing relies on knowledge transfer. "Learning" in prior work on the design of reasoning-based diagnosis engine can be viewed as direct self-learning [23, 40]. The diagnosis engine acquires knowledge from the failing boards of its own boardtype. Self-learning is useful when boards are in volume production, but it is less effective when an adequate number of failing boards is not available to learn from at the ramp-up stage. Therefore, the proposed knowledge-transfer method can facilitate knowledge inheritance from a mature diagnosis engine to the newer-diagnosis engine, such that the new diagnosis engine can not only learn from its own failing boards with more intelligence, but also from other boards. To enable knowledge transfer, the cases from the previous diagnosis engine are firstly abstracted as descriptive knowledge. The descriptive knowledge is then interpreted in the context of the new diagnosis engine for learning. In practice, the sources of knowledge transfer can not 
only be several old boards that have similar functionality as the new board, but also a limited number of failing cases from the new board itself.

The knowledge-transfer method is based on topological analysis. To facilitate knowledge reuse, we divide the knowledge transfer into three stages: (i) hardware mapping, (ii) test-program mapping, and (iii) case-knowledge transfer. The first two stages are reflected as root-cause mapping and syndrome mapping, respectively. The similarity between each component-pair of the old board and the new board is characterized by the functionality of the component on the board, while the similarity between test programs of the old and the new board is reflected by the topological characterization of the components covered by the test. Based on the root-cause mapping and syndrome mapping, the prior knowledge in the form of cases can be transferred to the new diagnosis system with a high degree of confidence.

We incorporate the synthetic cases generated from both knowledge discovery and knowledge transfer for training a diagnosis system. The weights of knowledge discovery and knowledge transfer are evaluated and discussed in detail in Section 7.5 .

\subsection{Knowledge-Discovery Method}

Board-level diagnosis relies on syndromes recorded in a test log. The extraction of fault syndromes is critical for model training in a diagnosis system. The fault syndromes should provide complete description about the fails, and the extracted syndromes for different actions should have sufficient diversity such that we can eliminate ambiguity in the eventual repair recommendations. Fault syndromes vary across products and tests. For example, a segment of the log file for a failed traffic test is shown in Figure 7.3. The fault syndromes extracted includes interrupt exception in SD12V_Master, mismatch errors in LAO Engine, and count error in SD23V, etc. Each of these elements is considered to be one syndrome. However, a traditional 


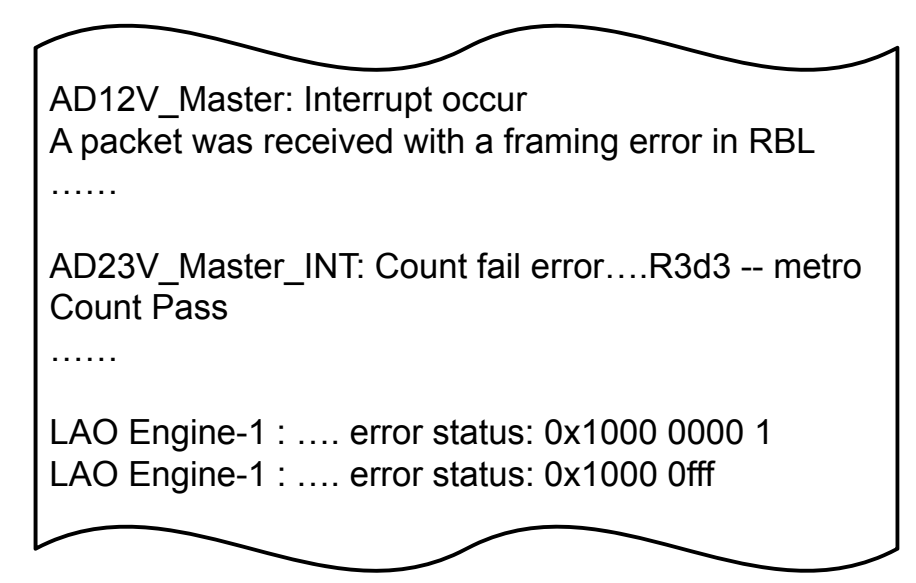

Figure 7.3: A segment of the log file for a failed functional test.

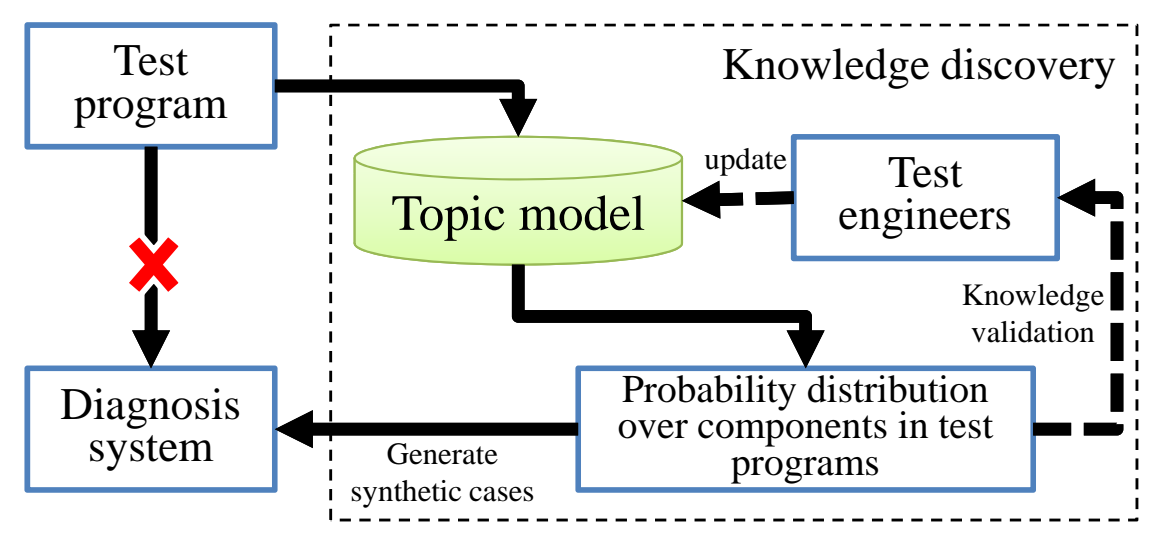

FiguRE 7.4: Flowchart of knowledge-discovery method.

diagnosis system treats each syndrome as a feature for classification and fails to provide any information for understanding the failing mechanism.

The proposed knowledge-discovery method mines valuable information from syndromes, since the syndromes designed by the debug engineer also contain information on underlying correlated root-causes. The proposed method follows a knowledgediscovery procedure $[80,81]$. The learning machine extracts the relationship between test programs and components. This relationship is validated and converted to knowledge by test engineers. The discovered knowledge in turn helps in the update of the learning machine. 


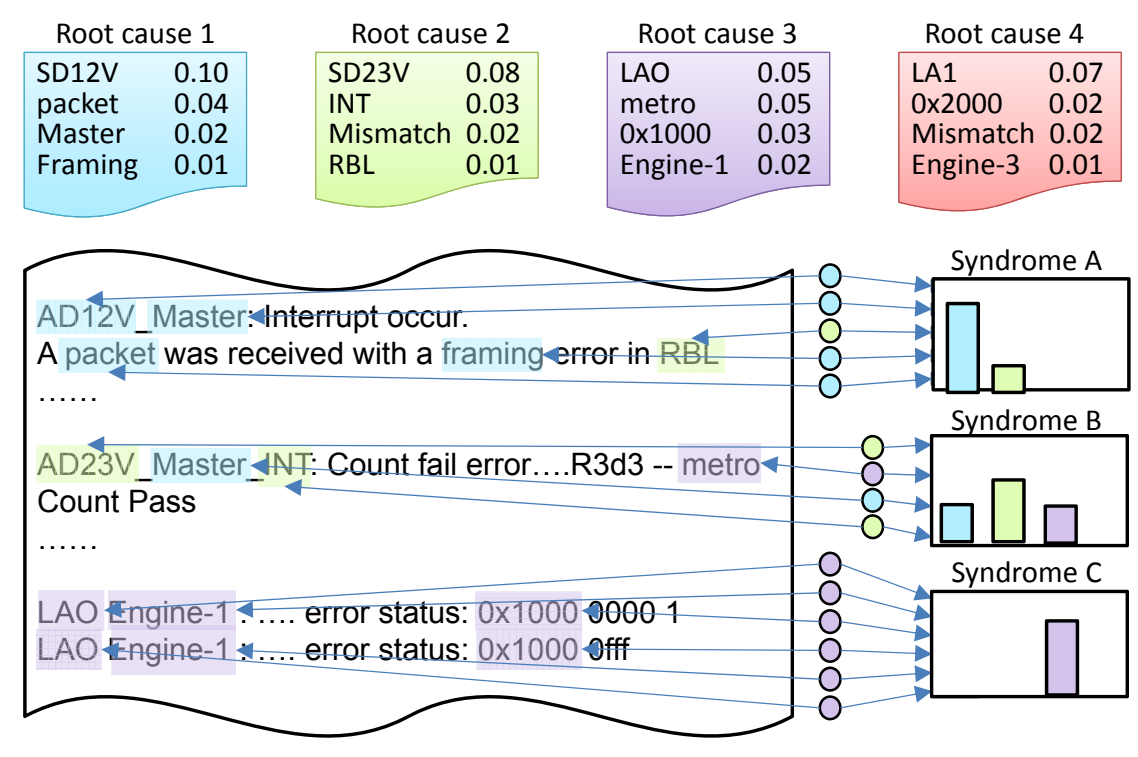

Figure 7.5: A segment of the log file for a failed functional test.

From the perspective of test design at board level, each component on a board is described or referred to using a knowledge pool of keywords. Each syndrome can be treated as a piece of text, which contains many single words. When the words in the syndrome match the keywords in the knowledge pool of a component, the syndrome is more likely to cover the root-cause. In contrast, if the syndrome does not contain any keyword related to the component, this syndrome is less likely to have the capability of isolating the root-cause. Figure 7.4 illustrates the use of the knowledge-discovery method. The relationship between root-causes and syndromes can be automatically revealed by a machine-learning technique called topic model. This relationship is represented in the form of a probability distribution involving components in test programs. Given such a probability distribution, test engineers can evaluate the relationship on the basis of which diagnosis system is trained. Moreover, test engineers can update the keyword list in the topic model based on the obtained probability distribution. Compared to rule-based or model-based methods that require significant manual effort from test engineers, the knowledge-discovery method significantly 
reduces the time needed to develop the diagnosis engine.

In machine learning or natural language processing, a topic model can discover the abstract "topics" that occur in a document [82]. Given a document about a particular topic, specific words about the topic tends to appear in the document with varying frequency. A document typically contains several topics in different proportions; thus the content on each topic may vary. A topic model can capture the proportion (a.k.a. probability) of topics in a document based on the statistics of words in the document and in the model for each topic. Our goal is to obtain $p\left(z_{j}\right)$, i.e., the probability that a document belongs to a topic $z_{j}$. Suppose that a document is regarded as a bag of words. The probability of each word $w_{i}$ in the document is $p\left(w_{i}\right)=\sum_{j=1}^{T} p\left(w_{i} \mid z_{j}\right) p\left(z_{j}\right)$, where $p\left(w_{i} \mid z_{j}\right)$ is the probability of word $w_{i}$ given the topic $z_{j}$ and $T$ is the total number of topics. Suppose there are a total of $N$ different words in $M$ documents, we include the probabilities of all words in an $N \times M$ matrix $P(w)$, which can be calculated as $P(w)=P(w \mid z) \times P(z)$, where $P(w \mid z)$ is a $N \times T$ matrix that corresponds to the probability distribution over words in each topic, and $P(z)$ is a $T \times M$ matrix corresponding to the probability distribution over topics in these documents. Therefore, $\mathrm{P}(\mathrm{z})$ can be calculated by

$$
P(z)=\Theta \times P(w),
$$

where $\Theta=\left(P(w \mid z)^{T} P(w \mid z)\right)^{-1} P(w \mid z)^{T}$. To apply such a topic model to board-level diagnosis, root-causes can be regarded as topics, while syndromes can be regarded as documents. The more keywords related to a root-cause occur in the syndrome description, the more likely is it that the root-cause can be isolated by the syndrome. For example, if a syndrome contains keywords "ECC" or "DDR", the syndrome tends to target a certain type of memory. If a syndrome contains keywords "packet" or "CRC", the syndrome tends to target network traffic engine.

Figure 7.5 gives an example of using knowledge discovery. Suppose we have a 
board that consists of four root-cause components, and each component has its keyword list and corresponding probabilities, respectively. We can extract the knowledge as a matrix $P(w \mid z)$. Moreover, we can extract probability distribution $P(w)$ over keywords from the log, as shown below:

$$
P(w)=\left[\begin{array}{ccc}
0.1 & 0 & 0 \\
0.1 & 0 & 0 \\
0.1 & 0.1 & 0 \\
0.1 & 0 & 0 \\
0 & 0.1 & 0 \\
0 & 0.1 & 0 \\
0 & 0 & 0 \\
0.1 & 0 & 0 \\
0 & 0 & 0.2 \\
0 & 0.1 & 0 \\
0 & 0 & 0.2 \\
0 & 0 & 0.2 \\
0 & 0 & 0 \\
0 & 0 & 0 \\
0 & 0 & 0
\end{array}\right] \quad P(w \mid z)=\left[\begin{array}{cccc}
0.1 & 0 & 0 & 0 \\
0.4 & 0 & 0 & 0 \\
0.2 & 0 & 0 & 0 \\
0.1 & 0 & 0 & 0 \\
0 & 0.8 & 0 & 0 \\
0 & 0.3 & 0 & 0 \\
0 & 0.2 & 0 & 0.2 \\
0 & 0.1 & 0 & 0 \\
0 & 0 & 0.5 & 0 \\
0 & 0 & 0.5 & 0 \\
0 & 0 & 0.3 & 0 \\
0 & 0 & 0.2 & 0 \\
0 & 0 & 0 & 0.7 \\
0 & 0 & 0 & 0.2 \\
0 & 0 & 0 & 0.1
\end{array}\right]
$$

where each row corresponds to a word. By using Equation (7.1), we can get $P(z)$ as below:

$$
P(z)=\left[\begin{array}{ccc}
0.36 & 0.09 & 0 \\
0.08 & 0.33 & 0 \\
0 & 0.13 & 0.52 \\
0 & 0 & 0
\end{array}\right]
$$

We can thus conclude that syndrome 1 has a probability of 0.36 correlated to root-cause 1 , a probability of 0.08 correlated to root-cause 2 , and no correlation with root-cause 3 and 4 . Similar syndrome and root-cause correlations can be obtained for the other two syndromes. By using the above knowledge-discovery technique, for each syndrome, a set of root causes can be automatically identified to relate to the syndrome. The proposed method considerably reduces manual labor when it is applied to rule-based and model-based methods, and it also provides an approach to collect knowledge that cannot be obtained in a traditional reasoning method. 


\subsection{Knowledge-Transfer Method}

Human learners appear to have inherent ways to transfer knowledge between two similar tasks. That is, we recognize and apply relevant knowledge from previous learning experiences when we encounter new tasks. The more related a new task is to our previous experience, the more easily we can master it. In the machine-learning domain, transfer learning is a challenging research problem; while we can enhance our knowledge of a task through transfer of knowledge from a related task that has already been learned, most machine-learning algorithms are designed to address only a single task [83].

In previous work on reasoning-based diagnosis, classical methods such as artificial neural networks, support vector machines, and decision trees [40,41] were used for training a diagnosis system for a board. Diagnosis systems for different boards, even for a board with only slighted updated test programs, must be trained separately. Although an automated training mechanism facilitates the development of a diagnosis system, the data acquisition process for a diagnosis system can be tedious and impractical in an industry setting. During the initial product ramp-up phase, reasoning-based diagnosis is need for yield learning, but the required database is not available due to lack of volume. Therefore, an intelligent diagnosis system, which can not only "learn" the knowledge from itself, but also from other diagnosis systems, can offer the important benefit of more effective knowledge acquisition at lower cost.

The knowledge-transfer process can be divided into three stages: 1) root-cause mapping, 2) syndrome mapping, and 3) case-knowledge transfer. This partitioning (see Figure 7.6) can be viewed as a realistic system functional-test design procedure as described below.

- Stage I mimics the board functionality design stage, where design engineers modify (i.e., add, remove, or change) the on-board components to get a new board design 


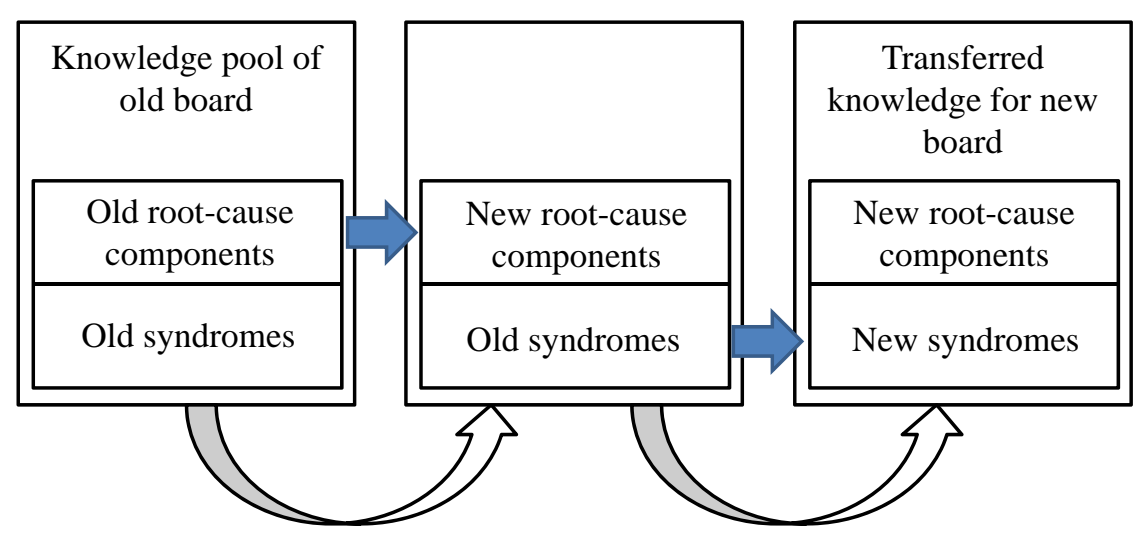

Step 1: Component mapping Step 2: Test-program mapping

Figure 7.6: Two-stage knowledge transfer from an old diagnosis system to a new diagnosis system.

in order to meet design specifications. In contrast, since the design-for-test team has not yet joined the design process at this stage, the test program remains the same as for the old board. In Stage I, only the board structure is transformed, while the test programs are simply inherited. In practice, we need to label the component according to its functionality to distinguish one component from another. For example, a component is marked as boot ROM when it is in charge of initial system start-up. Another component is marked as CPU external memory when it is a ROM or RAM device connected to a CPU. Although components may vary from generation to generation or from board to board, those components with the same functionality label are prone to suffer similar functional failures. Therefore, we can reconstruct the board structure conceptually by leveraging functionality labels for components. For example, in Figure 7.7 and Figure 7.8, a component is labeled as ASIC B in Figure 7.7. A similar component labeled as ASIC Bn can be found in Figure 7.8. Although two components on the two board differ from each other in term of the manufacturer or provider, operating frequency, or number of pins, these two components play the same role on the board. We therefore conclude that ASIC 


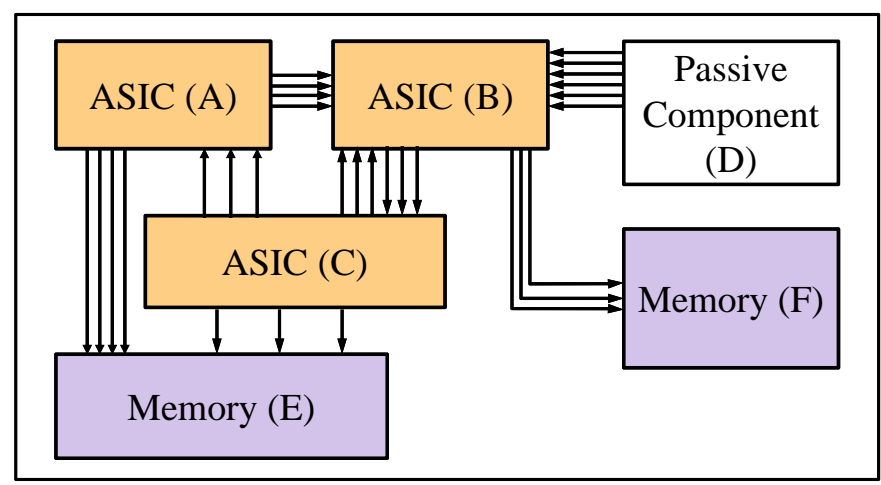

FigURE 7.7: Illustration of a mature board from which knowledge can be transferred.

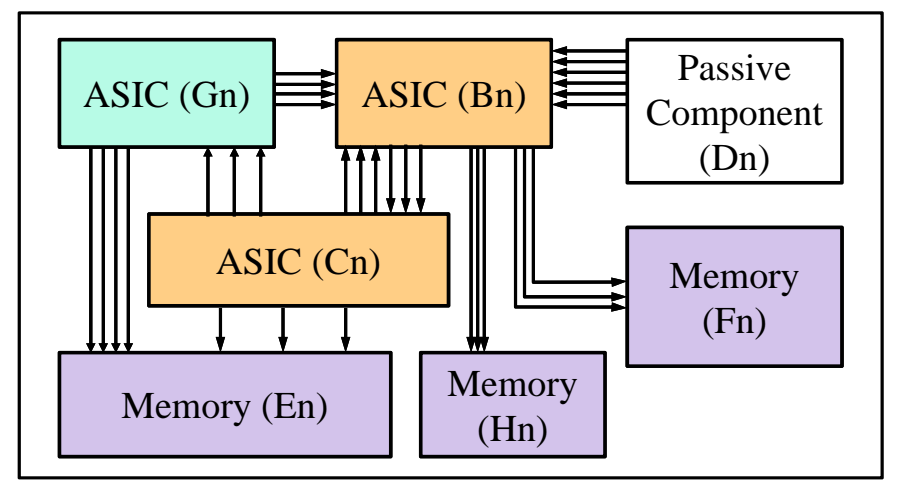

FigURE 7.8: Illustration of a new board that needs knowledge transfer.

B can be mapped to ASIC Bn, and thus the knowledge about ASIC B can be mapped to ASIC Bn.

In knowledge transfer, there are four types of relationships between components on the old board and those on the new board,

1. Suppose a component on the new board has exactly the same functionality label as on the old board. We can then map the component from the old board to the new board. Examples include ASIC B in Figure 7.7 vs. ASIC Bn in Figure 7.8, and also Memory F in Figure 7.7 vs. Memory Fn and Hn in Figure 7.8 .

2. If the functionality provided by a component on the new board was not imple- 


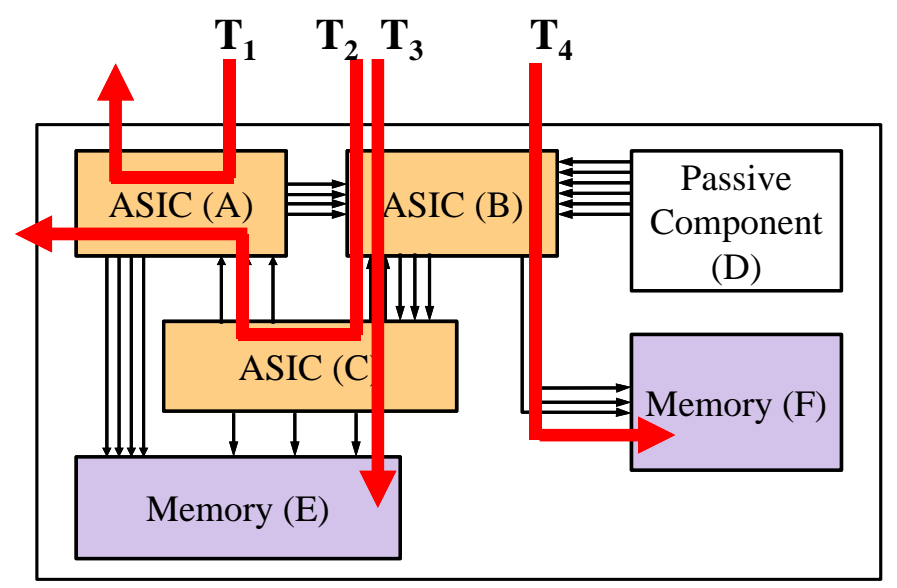

FIGURE 7.9: Illustration of tests on a mature board with knowledge to transfer from.

mented on the old board, and is therefore reflected as a new functionality label on a new board, we regard it as a new type of a root-cause. Clearly, there is no knowledge of such type of a root-cause on the old board that can be transferred to the new board. For example, in Figure 7.8, ASIC Gn is a new component on the new board, and it differs from any component on the old board.

3. A component on the old board is removed and the corresponding functionality of this component is not supported on the new board. The knowledge with regard to this component can no longer be transferred to the new board. For example in Figure 7.7, ASIC A is a component on the old board that no longer exists on the new board.

4. A component on the new board has only partial functionality of the component on the old board, or the functionality of a component on the new board is a combination of several components on the old board. The functionality of the component on the new board is different from those on the old board. Therefore, the knowledge of this type of component cannot be transferred.

- Stage II mimics the test-program development stage. In this stage, the function- 


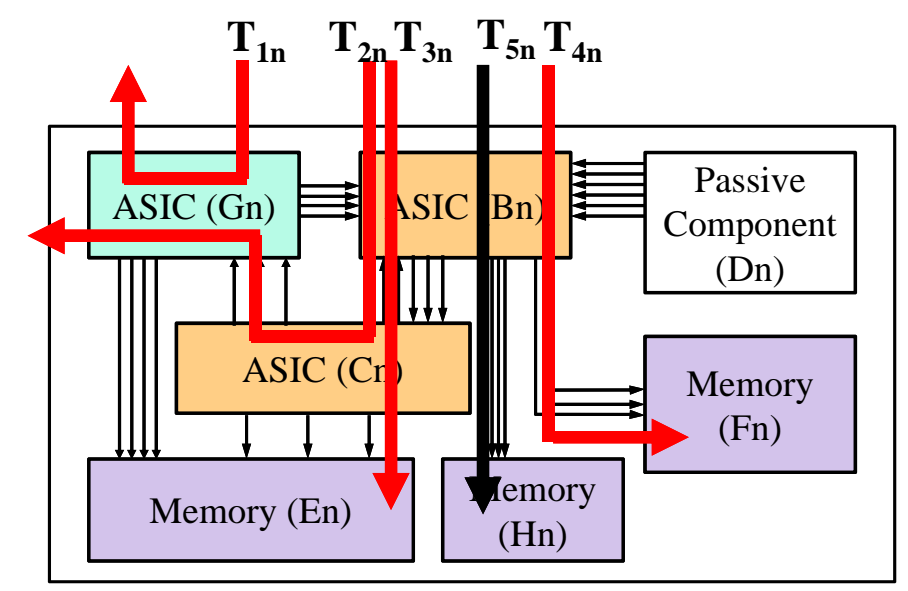

FiguRE 7.10: Illustration of tests on a new board that needs knowledge transfer.

ality has already met the board-design specifications. In order to assist design for test/diagnosis, the test design team joins the design process to develop new test program for the new board structure. We focus here on the changes to the test programs, while the board structure is considered to be fixed. Given the condition that two boards are similar in terms of functionality, knowledge about most of the test programs for the old board can still be used for those for the new board. Consider the examples shown in Figure 7.9 and Figure 7.10. ASIC B and Memory F on the old board in Figure 7.9 have similar components-ASIC Bn and Memory Fn-on the new board in Figure 7.10. Therefore, test $\mathrm{T}_{4}$ for the old board can be viewed as being similar test $\mathrm{T}_{4 \mathrm{n}}$ for the the new board. We conclude therefore that $\mathrm{T}_{4}$ can be mapped to $\mathrm{T}_{4 n}$, thus the knowledge about $\mathrm{T}_{4}$ can be transferred to $\mathrm{T}_{4 \mathrm{n}}$.

In addition, there are three types of transformations for test-program development, as described below:

1. If a test for the new board covers components with the same set of functionality labels as on the old board, we conclude that the knowledge of this test can be mapped to the new test program. If a case from the old board refers to a fail with the syndrome collected from this test, we can easily find a similar test for 


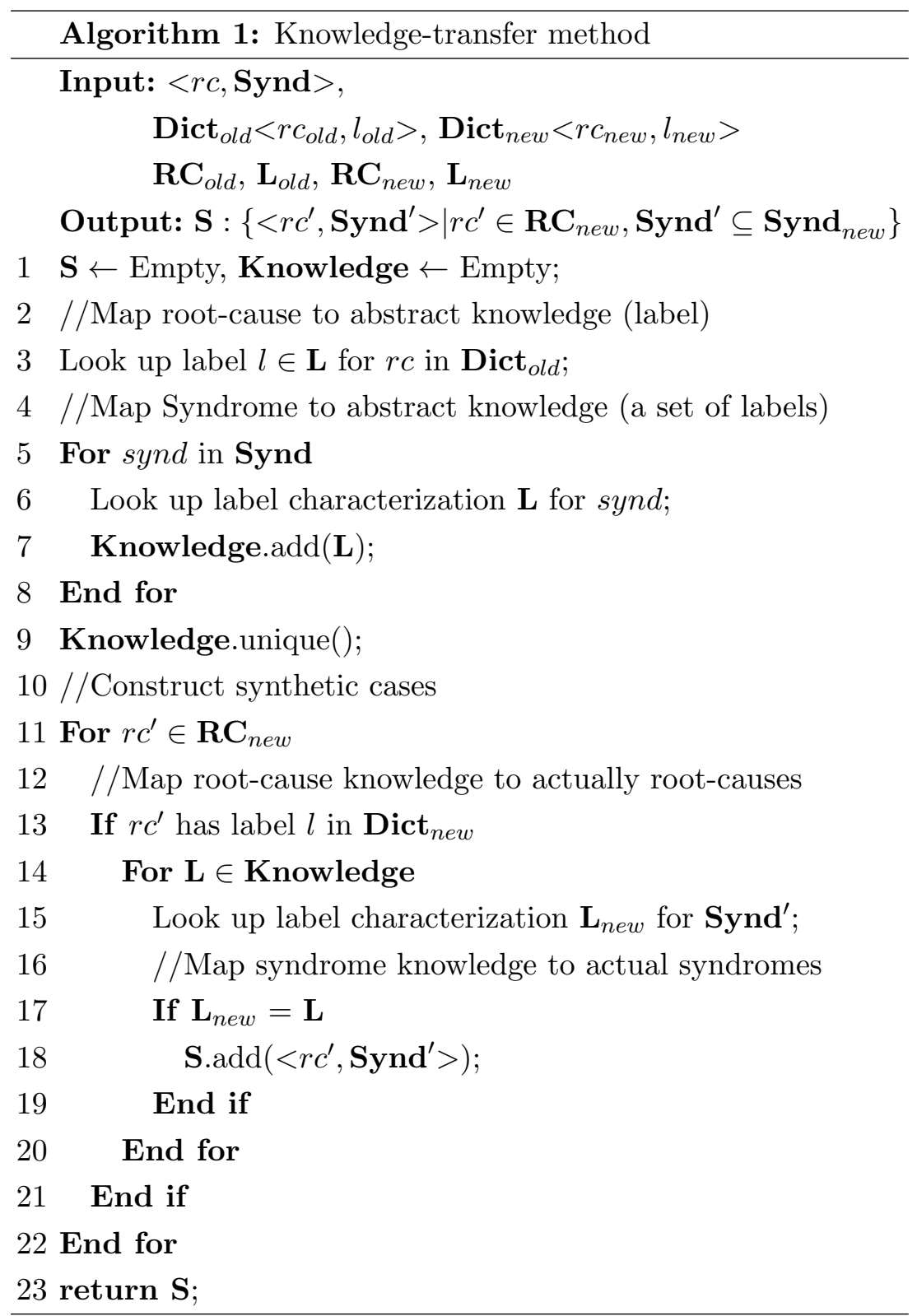

FiguRE 7.11: Proposed knowledge-transfer algorithm.

the new board. For example, $\mathrm{T}_{4}$ for the old board in Figure 7.9 and $\mathrm{T}_{4 \mathrm{n}}$ for new board in Figure 7.10 correspond to such a pair. Also, $\mathrm{T}_{3}$ can be mapped to $\mathrm{T}_{3 \mathrm{n}}$ since both tests covers two ASICs and one memory chip of the same type. Note that $T_{5 n}$ for the new board in Figure 7.10 can also be mapped to $T_{4}$, because Fn and Hn can both be labeled with Memory and they are connected 
to $\mathrm{Bn}$, resulting in both devices sharing the same topological characteristics.

2. If a test $\mathrm{T}_{i}$ is designed exclusively for the new board and targets new components, we regard this test as being new. No knowledge about the tests for the old board can be transferred to $\mathrm{T}_{i}$ in the new test program. For example, in Figure $7.10, \mathrm{~T}_{2 \mathrm{n}}$ and $\mathrm{T}_{1 \mathrm{n}}$ are both new because the component $\mathrm{Gn}$ covered by $\mathrm{T}_{2 \mathrm{n}}$ and $\mathrm{T}_{1 \mathrm{n}}$ is new.

3. If a test $\mathrm{T}_{i}$ covers components that exists only on the old board, knowledge about $\mathrm{T}_{i}$ must not be transferred to the new board to avoid biased or misleading knowledge. Examples include $t_{2}$ and $t_{1}$, as shown in Figure 7.9.

- Stage III leverages the root-cause mapping and syndrome-mapping information obtained from Stage I and II. The root-cause and syndromes for a case can be abstracted in terms of functionality labels at a higher knowledge level. Thus, the abstract knowledge can be interpreted in the context of the new board to generate synthetic cases with high confidence.

Figure 7.11 describes the knowledge-transfer algorithm. We are given a real case $<r c$, Synd $>$ collected from the old board, where $r c$ is the root-cause and Synd refers to a set of syndromes for the failing board. Our goal is to generate a set of synthetic cases $\mathbf{S}:\left\{\left\langle r c^{\prime}, \mathbf{S y n d}^{\prime}\right\rangle \mid r c^{\prime} \in \mathbf{R C}_{\text {new }}, \mathbf{S y n d}^{\prime} \subseteq \mathbf{S y n d}_{n e w}\right\}$ for the new board, where $\mathbf{R C}_{n e w}$ is the root-cause set and Synd $_{n e w}$ is the syndrome set for the new board, respectively. We have two dictionaries that consist of the components and the corresponding labels, i.e., Dict old $<r c_{\text {old }}, l_{\text {old }}>$ for the old board and $\mathbf{D i c t}_{\text {new }}<r c_{\text {new }}, l_{\text {new }}>$ for the new board, respectively, where each root-cause is paired to its corresponding functionality label.

First, we carry out root-cause mapping. The functionality label of root-cause $r c$ is

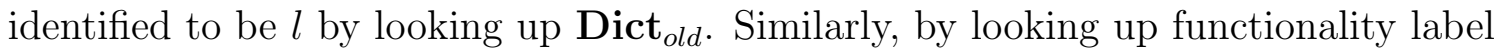
$l$ in Dict $_{n e w}$ for the new board, a set of root-causes $\left\{r c_{n e w} \mid<r c_{n e w}, l>\in\right.$ Dict $\left._{n e w}\right\}$ 
can be found with the same functionality label $l$. Next, we proceed with syndrome mapping. Each syndrome synd $\in$ Synd from the case can be mapped to synd $_{\text {new }}$ for the new board based on label characterization. A label-characterization procedure refers to description of a syndrome in the form of a set of functionality labels $\mathbf{L}$. We need to find a set of syndromes Synd' for the new board with the same label characterizations as that of Synd for the old board. Due to the fact that multiple components on the new board can have the same functionality label $l$, multiple syndromes synd ${ }_{\text {new }}$ may have the same label characterization $\mathbf{L}_{\text {new }}$. A set of synthetic cases $\mathbf{S}$ can be generated for the new board by assuming that root-cause $r_{\text {new }}$ has the same functionality label $l$ as that of the root-cause on the old board. Moreover, the corresponding syndromes for the synthetic case have the same label characterization as that of the syndromes for the old board. Assuming that the number of syndromes for an actual case is much less than the total number of syndromes, the computation complexity of the algorithm in Figure 7.11 is $\mathrm{O}(\mathrm{UV})$, where $\mathrm{U}$ is the total number of syndromes and $\mathrm{V}$ is the total number of root-causes for the new board, respectively.

We illustrate the knowledge-transfer method with an example. Suppose that we have a failing board from the old board with the structure shown in Figure 7.7 and the test program in Figure 7.9. A failing case has a root-cause - memory F, and the corresponding syndrome is a "fail" for $\mathrm{T}_{4}$. Thus, the case to be transferred is $<\mathrm{F}, \mathrm{T}_{4}>$. First, we use root-cause mapping. The components Fn and Hn on the new board have the same functionality label "Memory" as F on the old board. Next, we carry out syndrome mapping. We find that tests $T_{4 n}$ and $T_{5 n}$ have the same functionality characterization as $\mathrm{T}_{4}$, because they both consist of a Memory component and a ASIC B component. Therefore, we can generate two synthetic cases $<\mathrm{Fn}, \mathrm{t}_{4 \mathrm{n}}>$ and $<\mathrm{Hn}, \mathrm{T}_{5 \mathrm{n}}>$, respectively during the knowledge-transfer step. 
Table 7.1: Information about the industrial boards with mature diagnosis engines.

\begin{tabular}{|c|c|c|}
\hline & Board 1' & Board $2^{\prime}$ \\
\hline Number of syndromes & 299 & 201 \\
\hline Number of repair candidates (components) & 153 & 116 \\
\hline Number of boards & 1903 & 1634 \\
\hline
\end{tabular}

Table 7.2: Information about the industrial boards at the ramp-up stage.

\begin{tabular}{|c|c|c|c|}
\hline & Board 1 & Board 2 & Board 3 \\
\hline Number of syndromes & 3474 & 5537 & 10884 \\
\hline Number of repair candidates (components) & 258 & 276 & 483 \\
\hline Number of boards & 274 & 193 & 245 \\
\hline
\end{tabular}

\subsection{Experimental Results}

\subsubsection{Experimental Setup}

Experiments were performed on five industrial boards, two of which are currently in high-volume production and the other three of which are in the ramp-up stage. Relevant information about the boards is provided in Table 7.1 and Table 7.2. For example, Board 1' in Table 7.1 has a mature diagnosis engine that has been maintained for nearly two year. A total of 1903 repaired boards were collected from the contract manufacturer's database. A total of 299 fault syndromes were extracted from failure logs. The number of faulty components identified in the database for repair action is 153 . Board 1 and Board 2 in Table 7.2 are the boards at the rampup stage with only a few failing boards. These boards share similar designs, where Board 1, 2, and 3 are the successor designs to Board $1^{\prime}$ and $2^{\prime}$. The design changes include increasing/decreasing network bandwidth, cost-driven board redesign with component changes, etc. Therefore, knowledge-transfer method can be applied to these boards.

The knowledge-discovery and knowledge-transfer algorithms are implemented using Python. We use several diagnosis engines for demonstration, including support- 
vector machines(SVMs), artificial neural-networks(ANNs), and decision trees(DTs) based on weka [84]. Experiments were run on a 64-bit Linux system with 12 GB of RAM and a quad-core Intel i7 processor running at $2.67 \mathrm{GHz}$.

In order to assess the performance of the knowledge-discovery and transfer methods, and its ability to accurately predict the root-cause of failure on a new board, we use the real cases collected for three new boards described in Table 7.2 to evaluate the diagnosis engines. To ensure real-time diagnosis and repair, we assume that we are allowed at-most three attempts to replace the potential failing components. Succuss ratio (SR) is the ratio of the number of correctly diagnosed cases to the total number of cases. For example, if ten failing cases are diagnosed, a SR of $70 \%$ means that seven out of ten cases are correctly diagnosed and the remaining three cases are incorrectly diagnosed. We define $\mathrm{SR}_{1}$ as the success ratio corresponding to the cases for which the board is deemed to have been successfully repaired only when the actual faulty component is identified and placed at the top of the list of root-cause candidates. We also define $\mathrm{SR}_{2}\left(\mathrm{SR}_{3}\right)$ as the success ratio corresponding to the case that a board is deemed to have been successfully repaired if the actual faulty component is in the first two (three) positions in the list of candidates.

\subsubsection{Results and Discussion}

In Figure 7.12-7.14, we evaluate the diagnosis results for Boards 1-3 by comparing the proposed knowledge-discovery and transfer framework to the original knowledge pool created manually by the test designers; we refer to the latter as the "Manual method". The creation of such a comprehensive knowledge pool takes considerable time - a week or more for a team of engineers working on a board design - and it requires several experienced technicians to maintain the knowledge pool for months or

more. "Knowledge discovery" uses the proposed knowledge-discovery method only (i.e., without knowledge transfer). "Knowledge transfer ${ }_{b=1}$ " uses the proposed knowl- 


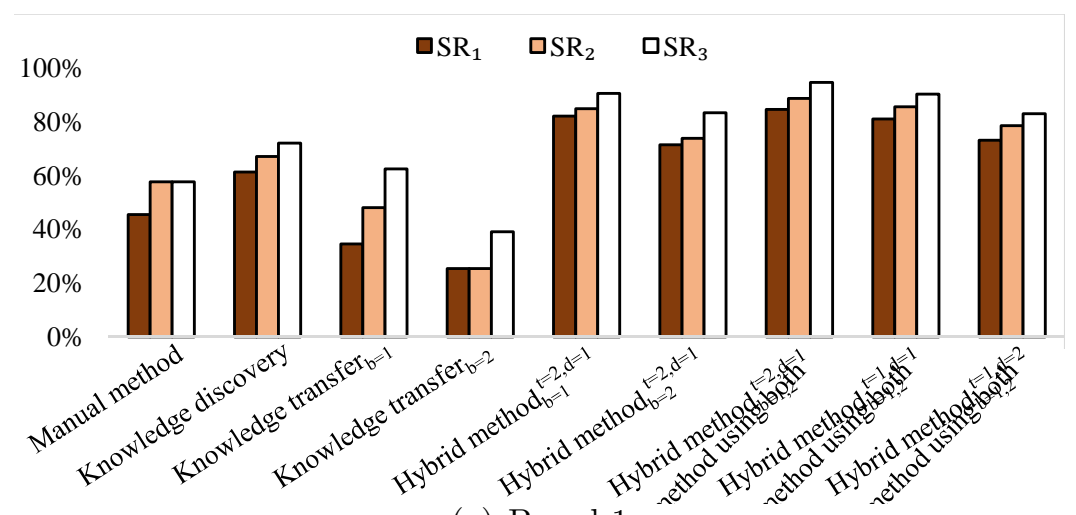

(a) Board 1

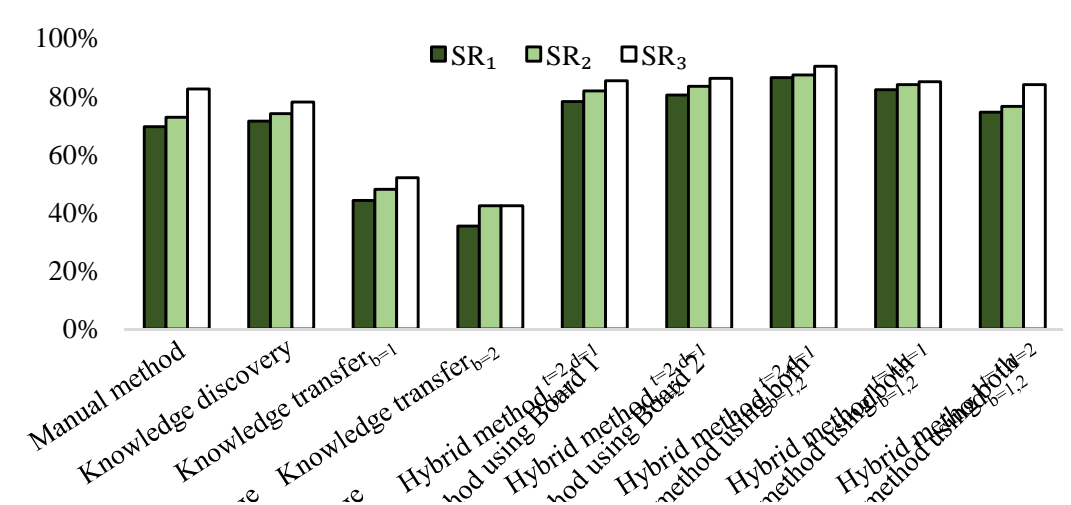

(b) Board 2

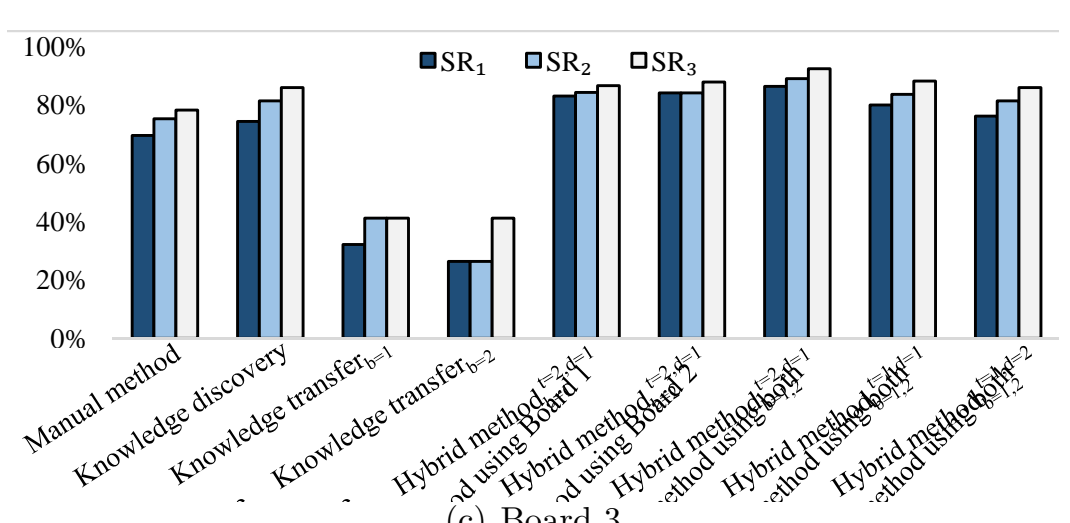

(c) Board 3

Figure 7.12: Comparison of diagnosis accuracy using SVMs between manual method, knowledge discovery, knowledge transfer, and the hybrid method.

edge transfer (i.e., without knowledge discovery) based on the knowledge from the mature diagnosis system for Board 1', and "Knowledge transfer ${ }_{b=2}$ " uses that from Board $2^{\prime}$ only. The hybrid method combines knowledge discovery and knowledge 


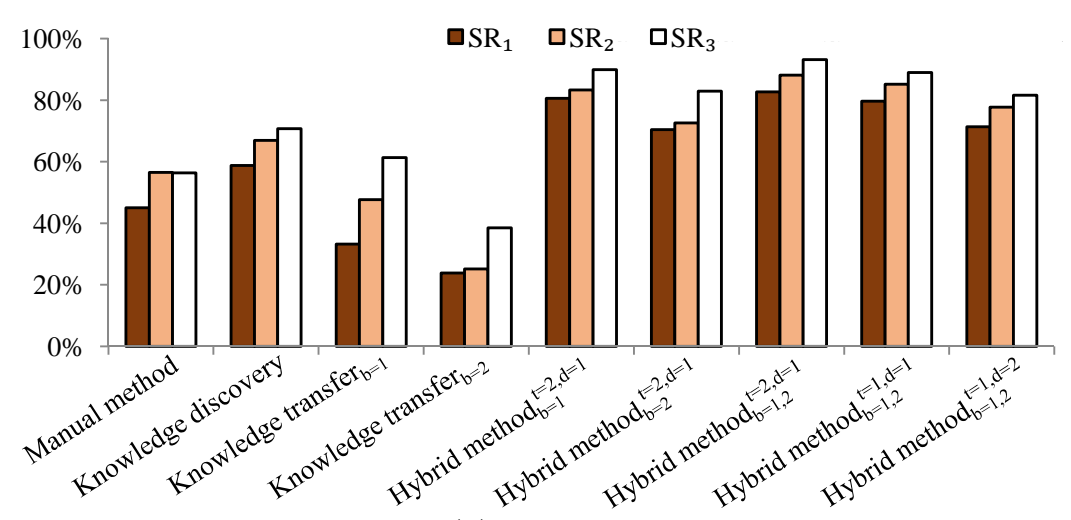

(a) Board 1

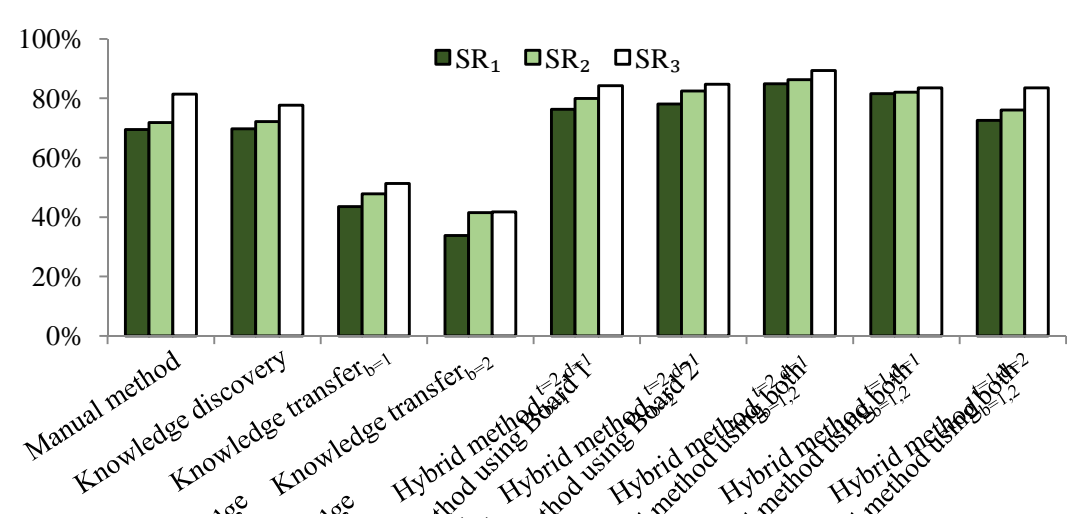

(b) Board 2

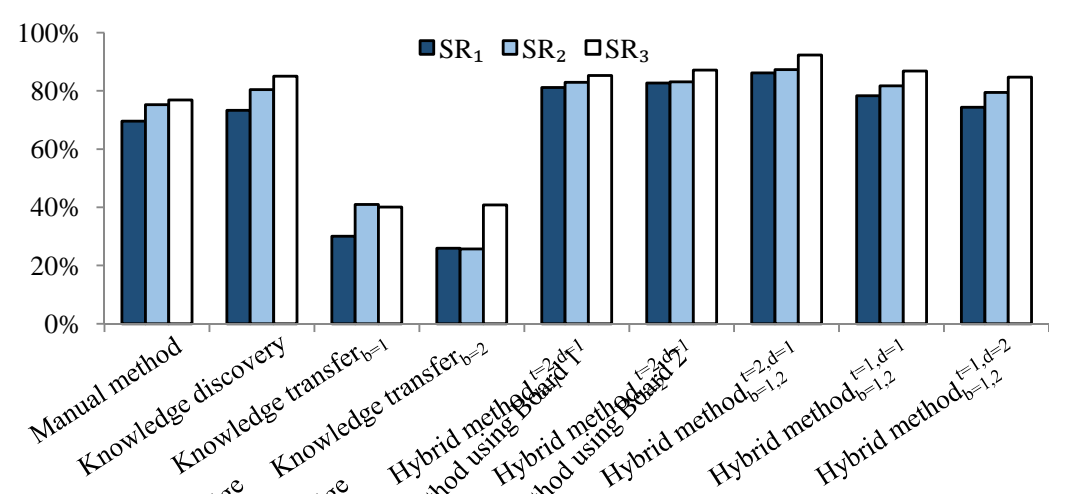

(c) Board 3

Figure 7.13: Comparison of diagnosis accuracy using ANNs between manual method, knowledge discovery, knowledge transfer, and the hybrid method.

transfer by using synthetic cases generated from both sources as shown in Figure 7.2; "Hybrid $\operatorname{method}_{b=1}$ " learns knowledge from Board 1' only; "Hybrid method ${ }_{b=2}$ " 


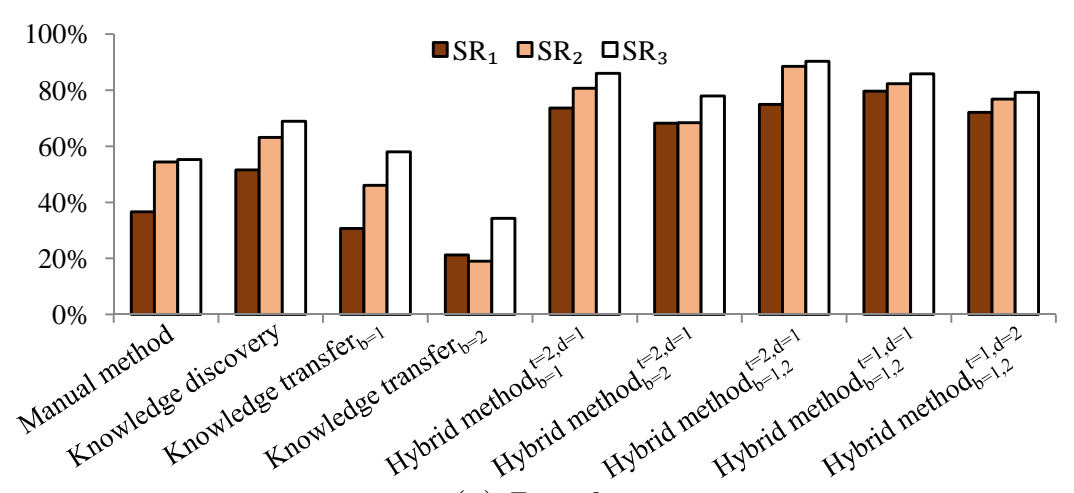

(a) Board 1

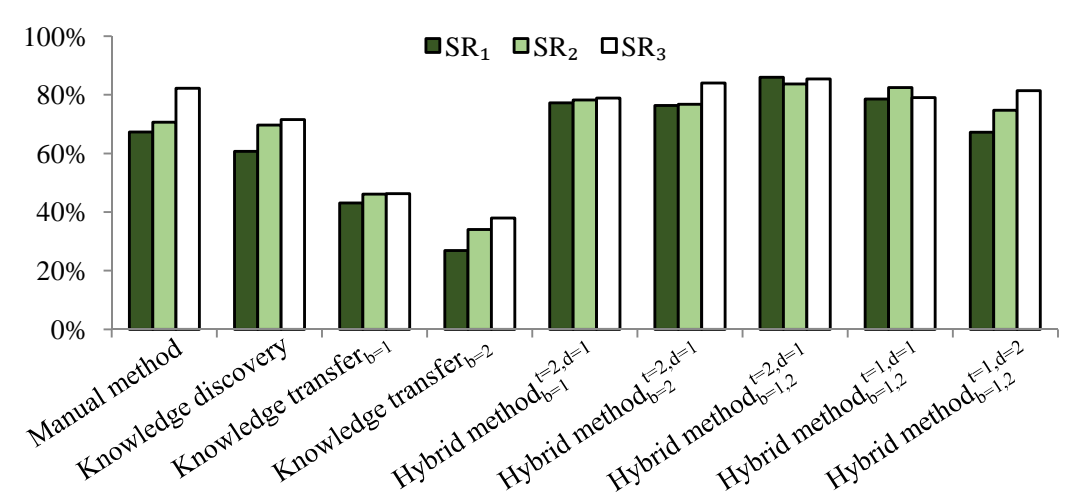

(b) Board 2

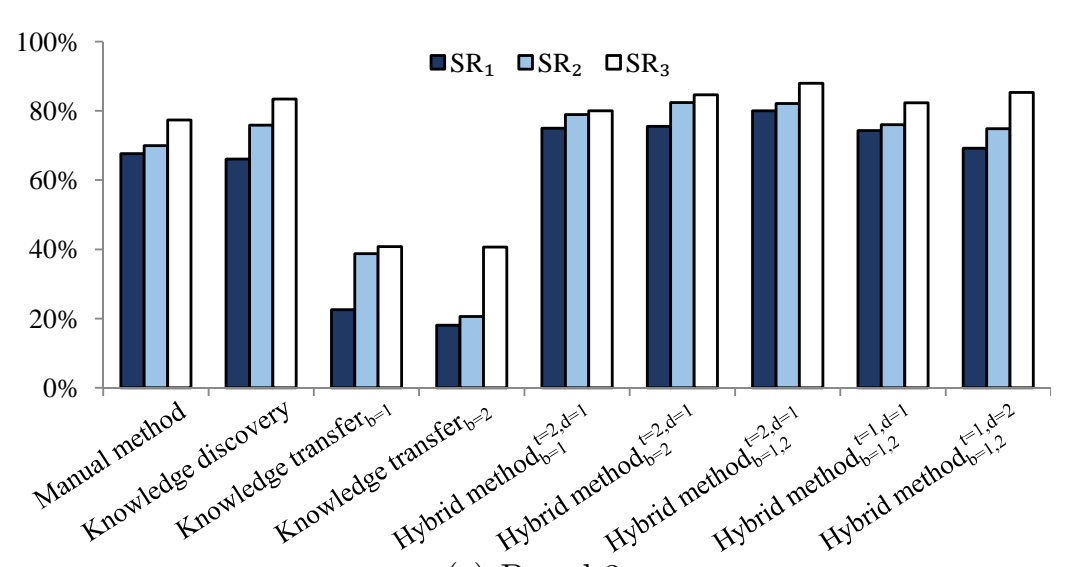

(c) Board 3

Figure 7.14: Comparison of diagnosis accuracy using DTs between manual method, knowledge discovery, knowledge transfer, and the hybrid method.

acquires knowledge from Board 2 ' only; and "Hybrid $\operatorname{method}_{b=1,2}$ " acquires knowledge from both Board $1^{\prime}$ and Board $2^{\prime}$. Moreover, we evaluate hybrid methods with 
different weight configurations for knowledge discovery and knowledge transfer. For example, "Hybrid method ${ }^{t=1, d=2 "}$ means the weight of synthetic cases generated using knowledge transfer is half of that using knowledge discovery; "Hybrid $\operatorname{method}^{t=1, d=1}$ " means the weights of synthetic cases generated using knowledge transfer and knowledge discovery are same; and "Hybrid method ${ }^{t=2, d=1 "}$ means the weight of synthetic cases generated using knowledge discovery is twice of that using knowledge discovery.

First, we evaluate the diagnosis performance of the knowledge-discovery method. Compared to the manual method, the diagnosis accuracy obtained by knowledgediscovery method is higher. For example, for the SVM-based diagnosis system for Board 1 in Figure 7.12(a), $\mathrm{SR}_{1}, \mathrm{SR}_{2}$, and $\mathrm{SR}_{3}$ are $61 \%, 67 \%$, and $72 \%$, respectively, using the knowledge-discovery method, compared to $45 \%, 57 \%$, and $57 \%$, respectively, using the manual method. An increase of $16 \%$ in $\mathrm{SR}_{1}$ is therefore obtained by using the knowledge-discovery method. Increases in success ratios are also observed in both Board 2 and Board 3. The reason that the knowledge-discovery method outperforms the manual method is that knowledge discovery avoids errors and biases that are likely to be introduced due to manual data input. Although debug engineers have many years of hands-on experience and are familiar with the board structure and test-program designs, the knowledge pool generated by the debug engineers is biased and incomplete, which can lead to low diagnosis accuracy. Moreover, due to the significant increase in the number of syndromes in test programs, it takes prohibitive long time to generate the knowledge pool manually. Nevertheless, knowledge-discovery method still suffers from the loss in diagnosis accuracy in that, in practice, error scenarios vary across boards and sometimes are not straightforward from the observation on syndromes. Such cases occurs in volume manufacturing and they can only be learned in reasoning-based diagnosis engines [39, 59].

Next, we evaluate the diagnosis accuracy achieved using the knowledge-transfer method. We observe that the diagnosis accuracy is comparable to manual method 
when we use "Knowledge-transfer ${ }_{b=2}$ " for training the diagnosis engines, while it is low when we use "Knowledge-transfer ${ }_{b=1}$ ". For example, the $\mathrm{SR}_{1}$ is $34 \%$ for "Knowledge-transfer ${ }_{b=1}$ " and the $\mathrm{SR}_{1}$ is $25 \%$ for "Knowledge-transfer ${ }_{b=2}$ " in Figure 7.12(a). The reason for the difference in diagnosis accuracies obtained using the two methods lies in the differences in board structure and test-program design. When we compare Board 1' to the three new boards, we find that a number of components on the new boards have different functionality labels from those on the old boards. On the other hand, the design of Board $2^{\prime}$ is closer to the designs of the three new boards, which makes the knowledge-transfer method more effective. Compared to the knowledge discovery and the manual method, knowledge transfer exploits the knowledge that was accumulated for the other boards in volume production. This knowledge can be transferred with high confidence, but the knowledge-transfer method is also limited by functional dissimilarities between the knowledge-source board and new board (e.g., it is impossible and meaningless to transfer the diagnosis knowledge from a linear-process unit to an embedded microcontroller.) Nevertheless diagnosis knowledge derived using the knowledge-transfer method is still valuable for training the diagnosis engine of new boards.

Then, we evaluate the hybrid method. First, we compare the hybrid methods with knowledge of different previous boards. For example, in the case of Board 1 in Figure 7.12(a), $\mathrm{SR}_{1}, \mathrm{SR}_{2}$, and $\mathrm{SR}_{3}$ are $84.5 \%, 88.5 \%, 94.5 \%$, respectively, for "Hybrid $\operatorname{method}_{b=1,2}^{t=2, d=1 "}$. Compared to the manual method, an increase of $39 \%$ in $\mathrm{SR}_{1}$ is obtained by using "Hybrid method $\operatorname{mon}_{b=1,2}^{t=2, d=1}$ ". The large increase in success ratios in $\mathrm{SR}_{1}, \mathrm{SR}_{2}$, and $\mathrm{SR}_{3}$ can also be observed for Board 2 in Figure Figure 7.12(b) and Board 3 in Figure Figure 7.12(c), respectively. The reason for this remarkable increase in diagnosis accuracy is that the hybrid method leverages the benefits of both knowledge discovery and knowledge transfer; the knowledge-discovery method 
captures the knowledge of relationship between syndromes and root-causes, while knowledge-transfer method utilizes knowledge from old boards that exhibit similar failures as the new board.

Next, we compare the hybrid method using different weight configurations. We observe that the diagnosis accuracy is higher when we assign higher weight to the knowledge-transfer method. For example, in the case of Board 1 in Figure 7.12(a), the $\mathrm{SR}_{1}$ are $83 \%, 81 \%$, and $74 \%$, respectively, for "Hybrid $\operatorname{method}_{b=1,2}^{t=2, d=1 "}$ ", "Hybrid $\operatorname{method}_{b=1,2}^{t=1, d=2 ",}$, "Hybrid $\operatorname{method}_{b=1,2}^{t=1, d=2}$ ", respectively. The reason for this observation is that knowledge transfer brings knowledge that has been successfully utilized for the earlier-generation boards with similar design. This knowledge reveals the inherent relationship between syndromes and root-causes, which cannot be obtained easily using the knowledge-discovery method or the manual method. The use of higher weight for knowledge transfer can help correct the diagnosis results.

In addition, we compare the diagnosis systems using statistical evaluation metrics, namely precision and recall, described in Chapter 6.

Figure 7.15 shows the precision and recall distributions for all the root-causes for the diagnosis system trained using different methods for Board 1. In each graph, the $\mathrm{x}$-axis corresponds to the value range of precision or recall, while the $\mathrm{y}$-axis corresponds to the number of root-causes that have the precision/recall value within the corresponding value range. A total of 258 root causes are available for detailed examination for Board 1. We observe that precision and recall values of root-causes for the hybrid methods are higher than the manual method. For example, the recall values of 188 root causes are 1 and the precision values of 179 root causes are 1 for "Hybrid method ${ }_{b=1,2}^{t=2, d=1 "}$; in contrast, the recall values of only 145 root causes are 1 and the precision values of 170 root causes are 1 for the manual method. Moreover, these values are considerably lower for knowledge transfer alone. For example, the 


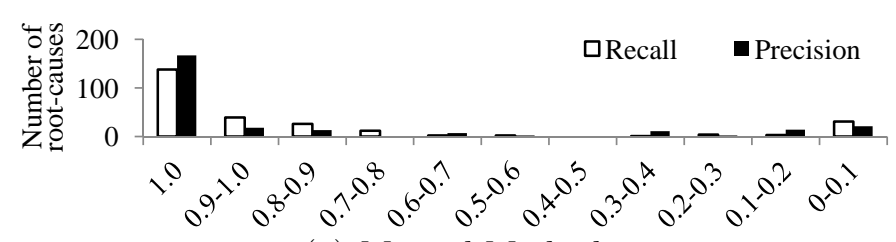

(a) Manual Method

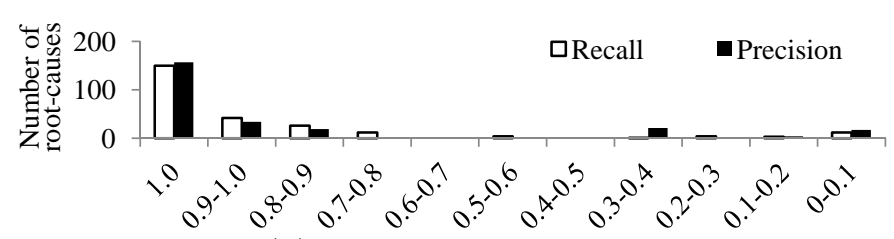

(b) Knowledge discovery

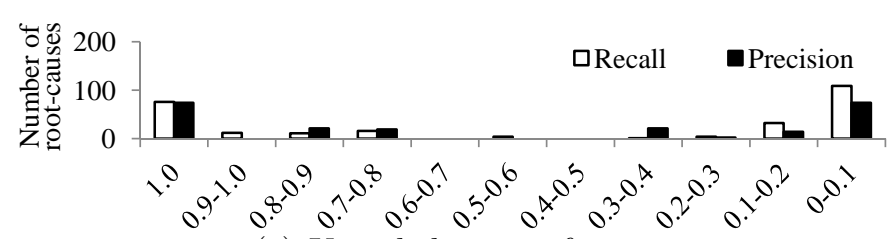

(c) Knowledge transfer ${ }_{b=1}$

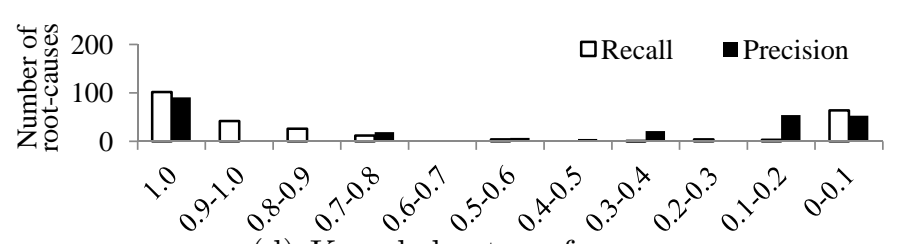

(d) Knowledge transfer ${ }_{b=2}$

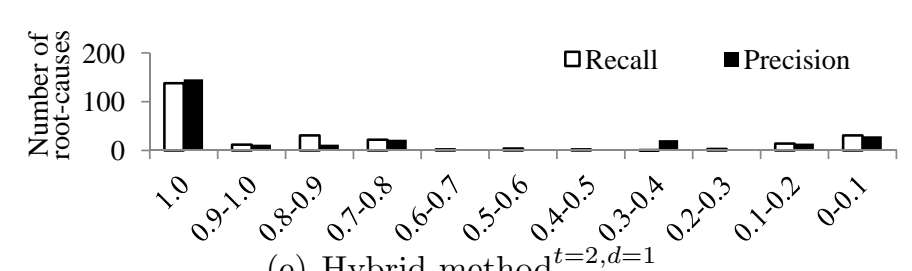

(e) Hybrid method ${ }_{b=1}^{t=2, d=1}$

Figure 7.15: Precision and recall distribution for an SVM-based diagnosis system for Board 1 (Part I).

precision values of 109 root causes are below 0.1 , and the recall values of 89 are below 0.1 for Knowledge transfer ${ }_{b=1}$. The reason for low precision and recall values can be attributed to the fact that most components on Board $1^{\prime}$ have different functionalities compared to Board 1. No knowledge can be transferred if components of similar 


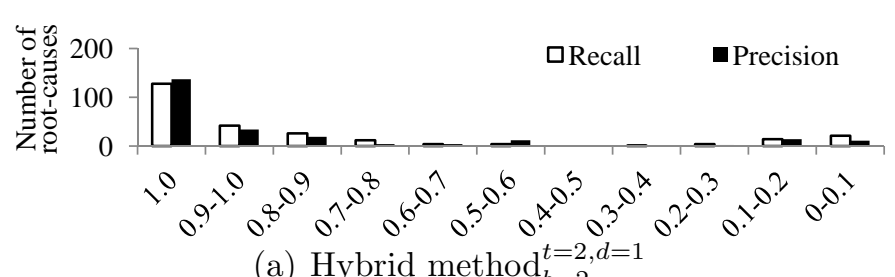

(a) Hybrid $\operatorname{method}_{b=2}^{t=2, d=1}$

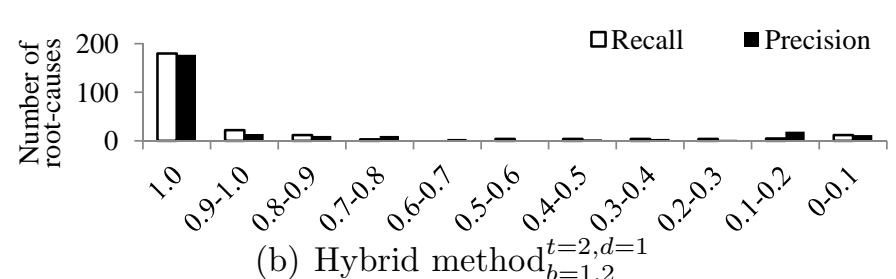

(b) Hybrid method ${ }_{b=1,2}^{t=2, d=1}$
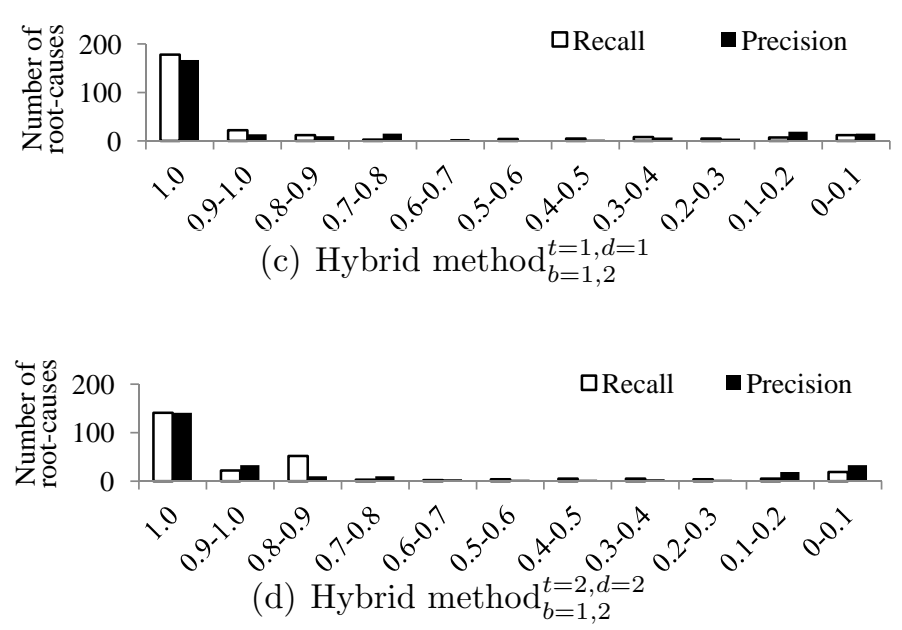

FiguRE 7.16: Precision and recall distribution for an SVM-based diagnosis system for Board 1 (Part II).

functionalities cannot be found on the new board.

We also compare the diagnosis accuracy obtained using difference types of diagnosis engines. We observed that the difference in diagnosis accuracy is not significant. The diagnosis performance of SVM-based diagnosis system outperforms that of ANN-based and DT-based diagnosis systems as illustrated in the literatures [40,41]. As shown in Figure 7.12-7.14, knowledge discovery and knowledge transfer are both effective for different types of diagnosis engines.

In addition, the knowledge-discovery method can also be useful in practice as a 
reference for debug engineers to evaluate the knowledge pool generated by the manual method. The proposed approach based on knowledge discovery and knowledge transfer is recommended for a reasoning-based diagnosis engine since it facilitates automation in diagnosis, therefore overcoming the bottleneck of "knowledge acquisition" at the product ramp-up stage.

\subsection{Conclusions}

Reasoning-based diagnosis method suffers low diagnosis accuracy due to the difficulty of "data acquisition" at the product ramp-up stage. This chapter has proposed a knowledge-discovery and knowledge-transfer framework to automatically build a knowledge pool for the new diagnosis engine from the information contained in the syndromes as well as the diagnosis experience collected from other diagnosis engines of similar board types. Five industrial boards have been used to demonstrate the effectiveness of using the knowledge-discovery and knowledge-transfer framework for root-cause identification in failing boards. 


\section{Conclusions and Future Work}

Advances in semiconductor technology have driven the electronics industry towards higher levels of integration with shorter development cycles. However, ever-increasing integration density and clock frequencies make it more difficult to ensure product quality at the board and system levels, which adversely impacts the competitiveness of a system manufacturer. Automated test and diagnosis systems are thus needed to ensure high quality and manage production cost. However, existing board-level testing and diagnostic strategies are insufficient to meet the requirements of the user community for products with high reliability. The development and maintenance of diagnosis systems using existing solutions require extensive human experience and manual labor. Moreover, todays diagnosis systems suffer low accuracy, which leads to an increase in then number of repair attempts and low product yield. The resulting high manufacturing cost motivates the need for an automated diagnosis system with high diagnosis accuracy. This thesis has presented a number of solutions to address pressing challenges in board-level functional diagnosis, which forms then core of an automated diagnosis system.

The goal of the research on intelligent diagnostic methods was to reduce the 
dependence of board-level diagnosis on time-consuming and ineffective human effort. Multiple machine learning and statistical methods have been studies and adapted for diagnosis. Substantial improvement has been achieved over currently deployed diagnostic software. The proposed solutions are not limited to a particular product; they are generic and can therefore be applied to various products. Although the goal of this thesis was to advance board-level diagnosis, the core techniques developed as part of this research can also be leveraged for larger electronic systems.

\subsection{Thesis Contributions}

The dissertation has significantly advanced board-level functional fault diagnosis. Recall that the diagnosis procedure involves several key components: (1) design of functional test programs, (2) collection of functional-failure syndromes, (3) building of the diagnosis engine, (4) isolation of root causes, and (5) evaluation of the diagnosis engine. The thesis research has targeted all the above five components of the diagnosis procedure.

Chapter 2 proposed a diagnosis system that extends a support-vector machinebased diagnosis engine. The proposed diagnosis system provides high accuracy and incorporates self-learning based on multi-kernel support-vector machines (MKSVMs). The MK-SVM method leverages a linear combination of single kernels to achieve accurate root-cause isolation. Incremental learning is used to continuously adapt the diagnosis system to newly occurring error scenarios.

Chapter 3 described an enhanced diagnosis system that leverages data fusion based on majority-weighted voting (MWV). The MWV-based diagnosis system can leverage multiple learning techniques (e.g., ANNs, SVMs, BIs, etc.) for diagnosis. It offers an attractive approach for aggregating decisions obtained from multiple diagnosis engines and can thus achieve high diagnosis accuracy.

Chapter 4 proposed an adaptive diagnosis system based on decision trees. Due 
to the large number of syndromes required to ensure diagnosis accuracy, the diagnosis time can be considerable for complex boards. The proposed adaptive diagnosis method aims to reduce the diagnosis time based on statistical analysis using decision trees. The number of syndromes required for diagnosis can thus be significantly reduced compared to the number of syndromes used for system training. Moreover, in order to boost the diagnosis accuracy at an early stage in volume production and to bridge the knowledge obtained at test-design stage with the knowledge gained during volume production, the DT structure has been enhanced to enable incremental learning.

Chapter 5 targeted the pre-processing of syndromes for diagnosis. Traditional diagnosis systems fail to provide appropriate repair suggestions when the diagnostic logs are fragmented and some syndromes are not available. The proposed diagnosis system adds the capability to handle syndromes based on imputation methods. The use of imputation methods help us to infer missing syndromes, so as to aid in the isolation of root-cause components for those failing boards that cannot be diagnosed using traditional diagnosis engines.

Chapter 6 described an evaluation and enhancement framework for guiding diagnosis systems. The proposed evaluation framework leverages syndrome and rootcause analysis based on information theory. Syndrome analysis based on subset selection provides a representative set of syndromes with minimum redundancy and maximum relevance. Root-cause analysis measures the discriminative ability of differentiating a given root cause from others. The metrics obtained from the proposed framework can not only grade the performance of the diagnosis system, but also provide guidelines for test redesign to enhance diagnosis.

Finally, Chapter 7 presented two knowledge-acquisition methods to tackle the difficulty of "data acquisition" during the initial product ramp-up phase. The proposed knowledge-discovery method and the knowledge-transfer method help enrich 
the training data set for training diagnosis systems, thus overcoming the bottleneck of low diagnosis accuracy at an early stage during volume production. First, knowledge-discovery method based on nature-language processing is used to automatically mine the relationship between syndromes and components from syndrome descriptions. Second, given the effectiveness of syndromes for root-cause mapping, knowledge from diagnosis engines used for earlier-generation products can be automatically transferred for training diagnosis systems for new products.

\subsection{Future Work}

Machine learning provides an important theoretical basis for advancing board/systemlevel test and diagnosis, especially as we move towards more complex products with even higher integration levels and production in high volume. Inefficient diagnosis based on manual debug and repair will eventually be replaced by automated diagnosis and repair. Machine learning and computer-aided statistical analysis provide a promising solution for diagnosis automation. This thesis research has opened up the following interesting new directions.

\section{- Multiple root-causes}

A single root-cause has been targeted for all failing boards in this thesis; in reality, however, complex systems often fail due to multiple failing components. In current diagnosis systems, multiple suspicious root-causes are listed for repair by technicians with the most-likely root cause at the top of the list. However, syndromes obtained from failing systems can indicate more than one root cause. Error scenarios for multiple root-causes cannot be handled using current diagnostic systems. It is important to develop a diagnosis system that can implicitly target multiple root causes. Such a system requires breakthroughs in training and diagnosis. In todays learning-based diagnosis systems, a learning case is formatted as a set of syndromes and a single 
root cause, but the desired multiple-root-cause diagnosis system must learn from experience in the form of a set of syndromes and multiple root causes.

\section{- Data mining in gate-level diagnosis}

As machine-learning techniques are increasingly adopted for board- and system-level diagnosis, we expect insights to emerge for utilizing machine learning afor chip- or gate-level test and diagnosis. Current state-of-the-art test and diagnosis techniques at gate-level mainly rely on structural tests, where it is assumed that we can preciously control chip voltages, temperatures, and signal levels. However, the processvariation profile varies for different chips, even if they are fabricated on the same wafer, and process variation is even more pronounced for different wafers. Different process variations lead to different error scenarios, which cannot be captured by structural tests. A data-driven reasoning-based system that utilizes machine learning can be a potential solution can be a solution for this problem.

The massive amount of data collected from the same product that are manufactured earlier can be used to train a reasoning system, which can be used for future test or diagnosis. Moreover, given the ability of collecting monitoring data from the chip under operation, the reasoning system thus trained can be used to monitor or predict chip performance on-the-fly. The prediction system can thus also incorporate aging effects. In this research direction, hardware implementation of machine learning techniques is another big concern. Trade-off in hardware design (i.e., silicon area, response timing, prediction accuracy, test or diagnosis function) should be carefully evaluated.

\section{- Diagnosis automation}

Although the diagnosis system studied in this thesis has involved considerable automation based on machine learning and artificial intelligence techniques, automation 
has not been fully realized for all the diagnosis steps. Research on automation for the following steps are needed:

1) Automatic identification of relationships between syndrome keywords to related components in knowledge discovery. A possible solution is to enable standard labeling for all components and analysis on board structural designs;

2) Automated improvement of tests based on evaluation framework. Current evaluation frameworks target the analysis of syndromes and root-causes with weak diagnosisability, while the test engineer must manually analyze the evaluation results to identify the appropriate test program for isolating the root cause with weak diagnosisabilbity from the remaining root-causes. By analyzing the information of board designs, it likely that appropriate test improvement can be automated. 


\section{Bibliography}

[1] Y.-T. Lin, "Economic designs for manufacturing system test and field maintenance," in Proceedings Southeastern Symposium on System Theory, pp. 40-44, 2005.

[2] C. O'Farrill, M. Moakil-Chbany, and B. Eklow, "Optimized reasoning-based diagnosis for non-random, board-level, production defects," in Proceedings IEEE International Test Conference (ITC), pp. 173-179, 2005.

[3] Z. Zhang, X. Gu, Y. Xie, Z. Wang, and K. Chakrabarty, "Diagnostic system based on support-vector machines for board-level functional diagnosis," in Proceedings IEEE European Test Symposium (ETS), 2012.

[4] Z. Zhang, K. Chakrabarty, Z. Wang, Z. Wang, and X. Gu, "Smart diagnosis: Efficient board-level diagnosis and repair using artificial neural networks," in Proceedings IEEE International Test Conference (ITC), 2011.

[5] T. Vo, Z. Wang, T. Eaton, P. Ghosh, H. Li, Y. Lee, W. Wang, H. Jun, R. Fang, D. Singletary, and X. Gu, "Design for board and system level structural test and diagnosis," in Proceedings IEEE International Test Conference (ITC), pp. 1-10, 2006 .

[6] S. Tourangeau and B. Eklow, "Test economics - What can a board/system test engineer do to influence supply operation metrics," in Proceedings IEEE International Test Conference (ITC), pp. 1-10, 2006.

[7] T. Chakraborty, C.-H. Chiang, and B. Van Treuren, "A practical approach to comprehensive system test \& debug using boundary-scan based test architecture," in Proceedings IEEE International Test Conference (ITC), pp. 1-10, 2007.

[8] D. Backstrom, G. Carlsson, and E. Larsson, "Remote boundary-scan system test control for the ATCA standard," in Proceedings IEEE International Test Conference (ITC), pp. 788-797, 2005. 
[9] W. R. Simpson and J. W. Sheppard, System Test and Diagnosis. Springer, 1994.

[10] M. Bushnell and V. Agrawal, Essentials of Electronic Testing for Digital, Memory, and Mixed-Signal VLSI Circuits. Springer, 2000.

[11] R. Isermann, Fault-Diagnosis Systems: An Introduction from Fault Detection to Fault Tolerance. Springer, 2006.

[12] R. Isermann, "Model-based fault-detection and diagnosis-status and applications," Annual Reviews in Control, vol. 29, no. 1, pp. 71-85, 2005.

[13] M. Abramovici, M. A. Breuer, and A. D. Friedman, Digital Systems Testing and Testable Design, vol. 2. Computer Science Press, New York, 1990.

[14] Y. Huang, R. McMurran, G. Dhadyalla, and R. P. Jones, "Probability based vehicle fault diagnosis: Bayesian network method," Journal of Intelligent Manufacturing, vol. 19, no. 3, pp. 301-311, 2008.

[15] W. R. Simpson and J. W. Sheppard, "System complexity and integrated diagnostics," Design 6 Test of Computers, IEEE, vol. 8, no. 3, pp. 16-30, 1991.

[16] IEEE Standard 1149.1, Test Access Port and Boundary-Scan Architecture. available at http://ieeexplore.ieee.org/stamp/stamp.jsp?tp= \&arnumber $=938734,2013$.

[17] IEEE Standard 1149.6, Boundary-Scan Tesing of Advanced Digital Networks. available at http://ieeexplore.ieee.org/stamp/stamp.jsp?tp= \&arnumber=1196298, 2013.

[18] I. Reis, P. Collins, and M. van Houcke, "Online boundary-scan testing in service of extended products," in Proceedings IEEE International Test Conference (ITC), 2006.

[19] K. Parker, "Defect coverage of boundary-scan tests: what does it mean when a boundary-scan test passes?," in Proceedings IEEE International Test Conference (ITC), pp. 181-189, 2003.

[20] B. Eklow, A. Hossein, C. Khuong, S. Pullela, T. Vo, and H. Chau, "Simulation based system level fault insertion using co-verification tools," in Proceedings IEEE International Test Conference (ITC), pp. 704-710, 2004. 
[21] Z. Conroy, G. Richmond, X. Gu, and B. Eklow, "A practical perspective on reducing ASIC NTFs," in Proceedings IEEE International Test Conference (ITC), 2005 .

[22] Mentor Graphics, Tessent Diagnosis. available at http://www.mentor.com/ products/silicon-yield/products/diagnosis, 2013.

[23] W. Fenton, T. McGinnity, and L. Maguire, "Fault diagnosis of electronic systems using intelligent techniques: A review," IEEE Transactions on Systems, Man, and Cybernetics, Part C: Applications and Reviews, vol. 31, pp. 269-281, 2001.

[24] P. H. Sydenham and R. Thorn, Handbook of Measuring System Design, vol. 2. Wiley, 2005.

[25] L. Amati, Test and diagnosis strategies for digital devices: methodologies and tools. PhD thesis, Politecnico di Milano, Italy, 2012.

[26] R. Boumen, S. Ruan, I. de Jong, J. Van De Mortel-Fronczak, J. Rooda, and K. Pattipati, "Hierarchical test sequencing for complex systems," IEEE Transactions on Systems, Man, and Cybernetics, Part A: Systems and Humans, vol. 39, no. 3, pp. 640-649, 2009.

[27] D. Manley and B. Eklow, "A model based automated debug process," in IEEE Board Test Workshop, 2002.

[28] H. Fang, Design-for-Testability and Diagnosis Methods to Target Unmodeled Defects in Integrated Circuits and Multi-Chip Boards. PhD thesis, Duke University, USA, 2011.

[29] Z. Zhang, Optimization of Fault-Insertion Test and Diagnosis of Functional Failures. PhD thesis, Duke University, USA, 2011.

[30] J. W. Sheppard and S. Butcher, "On the linear separability of diagnostic models," in IEEE Autotestcon, pp. 626-635, 2006.

[31] A. Darwiche, "Model-based diagnosis using structured system descriptions," Journal of Artificial Intelligence Research, vol. 8, pp. 165-222, 1998.

[32] T. Hastie, R. Tibshirani, and J. J. H. Friedman, The Elements of Statistical Learning. Springer, 2001. 
[33] A. A. Al-Jumah and T. Arslan, "Artificial neural network based multiple fault diagnosis in digital circuits," in Proceedings International Symposium on Circuits and Systems (ISCAS), vol. 2, pp. 304-307, 1998.

[34] S. Butcher and J. W. Sheppard, "Distributional smoothing in bayesian fault diagnosis," IEEE Transactions on Instrumentation and Measurement, vol. 58, no. 2, pp. 342-349, 2009.

[35] M. Catelani and A. Fort, "Soft fault detection and isolation in analog circuits: Some results and a comparison between a fuzzy approach and radial basis function networks," IEEE Transactions on Instrumentation and Measurement, vol. 51, no. 2, pp. 196-202, 2002.

[36] C. Pous, J. Colomer, J. Melendez, and J. L. de la Rosa, "Case base management for analog circuits diagnosis improvement," in Case-Based Reasoning Research and Development, pp. 437-451, Springer, 2003.

[37] F. Ye, Z. Zhang, K. Chakrabarty, and X. Gu, "Information-theoretic syndrome and root-cause analysis for guiding board-level fault diagnosis," in Proceedings IEEE European Test Symposium (ETS), 2014.

[38] Z. Sun, L. Jiang, Q. Xu, Z. Zhang, Z. Wang, and X. Gu, "AgentDiag: An agentassisted diagnostic framework for board-level functional failures," in Proceedings IEEE International Test Conference (ITC), pp. 1-8, 2013.

[39] C. Bolchini, E. Quintarelli, F. Salice, and P. Garza, "A data mining approach to incremental adaptive functional diagnosis," in Proceedings IEEE International Symposium on Defect and Fault Tolerance in VLSI Systems, pp. 13-18, 2013.

[40] F. Ye, Z. Zhang, K. Chakrabarty, and X. Gu, "Board-level functional fault diagnosis using artificial neural networks, support-vector machines, and weightedmajority voting," IEEE Transactions on Computer-Aided Design of Integrated Circuits and Systems (TCAD), vol. 32, no. 5, pp. 723-736, 2013.

[41] F. Ye, Z. Zhang, K. Chakrabarty, and X. Gu, "Adaptive board-level functional fault diagnosis using decision trees," in Proceedings IEEE Asian Test Symposium (ATS), pp. 202-207, 2012.

[42] P. Parvathala, K. Maneparambil, and W. Lindsay, "FRITS - a microprocessor functional BIST method," in Proceedings IEEE International Test Conference (ITC), pp. 590-598, 2002. 
[43] Z. Zhang, Z. Wang, X. Gu, and K. Chakrabarty, "Physical-defect modeling and optimization for fault-insertion test," IEEE Transactions VLSI System, vol. 20, no. 4, pp. 723-736, 2012.

[44] International Technology Roadmap for Semiconductors (ITRS'13). available at http://www.itrs.net/, 2013.

[45] Xbox 360 technical problems "Ring of Death". available at http://en. wikipedia.org/wiki/Xbox_360_technical_problems, 2007.

[46] Outages hit Amazon's S3 storage service. available at http://www . networkworld.com/news/2008/072108-amazon-outages.html, 2008.

[47] Cisco Line Cards. available at http://www.cisco.com/en/US/products/hw/ modules/ps2710/prod_module_series_home.html, 2013.

[48] iNEMI Technolog Roadmaps, from International Electronics Manufacturing Initiative Organazition. available at http://www. inemi .org/node/2151, 2009.

[49] Z. Zhang, Z. Wang, X. Gu, and K. Chakrabarty, "Board-level fault diagnosis using Bayesian inference," in Proceedings IEEE VLSI Test Symposium (VTS), 2010 .

[50] V. Vapnik, The Nature of Statistical Learning Theory. Springer, 1995.

[51] A. Rakotomamonjy and S. Canu, SVM and Kernel Methods Matlab Toolbox. available at http://asi.insa-rouen.fr/enseignants/ arakoto/toolbox/ index.html, 2008.

[52] G. Lanckriet, T. De Bie, N. Cristianini, M. Jordan, and W. Noble, "A statistical framework for genomic data fusion," Bioinformatics, vol. 20, pp. 2626-2635, 2004.

[53] M. Varma and B. R. Babu, "More generality in efficient multiple kernel learning," in Proceedings of ACM International Conference on Machine Learning (ICML), pp. 1065-1072, 2009.

[54] A. Rakotomamonjy, Y. Grandvalet, F. Bach, and S. Canu, "SimpleMKL," Journal of Machine Learning Research, vol. 9, pp. 2491-2521, 2008.

[55] T. Chang, H. Liu, and S. Zhou, "Large scale classification with local diversity AdaBoost SVM algorithm," Journal of Systems Engineering and Electronics, vol. 20, no. 6, pp. 1344-1350, 2009. 
[56] Incremental SVM Learning with multiclass support and probabilistic output. available at http://www-ti.informatik.uni-tuebingen.de/spueler/ mcpIncSVM/,2013.

[57] Neural Network Toolbox. available at http: //www . mathworks. com/products/ neuralnet/, 2012.

[58] G. McLachlan, K. Do, and C. Ambroise, Analyzing Microarray Gene Expression Data, vol. 422. John Wiley \& Sons, 2004.

[59] F. Ye, Z. Zhang, K. Chakrabarty, and X. Gu, "Board-level functional fault diagnosis using learning based on incremental support-vector machines," in Proceedings IEEE Asian Test Symposium (ATS), pp. 208-213, 2012.

[60] K. Totton and P. Limb, "Experience in using neural networks for electronic diagnosis," in ACM International Conference on Artificial Neural Networks, pp. 115-118, 1991.

[61] S. Haykin, Neural Networks and Learning Machines. Prentice Hall, New Jersey, 2008.

[62] N. Littlestone and M. Warmuth, "The weighted majority algorithm," Journal Information and Computation, vol. 108, no. 2, pp. 212-261, 1994.

[63] S. Keerthi and C. Lin, "Asymptotic behaviors of support vector machines with Gaussian kernel," Neural Computation, vol. 15, no. 7, pp. 1667-1689, 2003.

[64] L. Breiman, Classification and Regression Trees. Chapman \& Hall/CRC, 1984.

[65] J. Quinlan, "Induction of decision trees," Machine Learning, vol. 1, no. 1, pp. 81$106,1986$.

[66] A. Vlahou, J. O. Schorge, B. W. Gregory, and R. L. Coleman, "Diagnosis of ovarian cancer using decision tree classification of mass spectral data," Journal of Biomedicine and Biotechnology, vol. 2003, no. 5, pp. 308-314, 2003.

[67] S. Abu-Hakima, "Visualizing and understanding diagnoses," Canadian Artificial Intelligence, vol. 30, 1992.

[68] Matlab Statistics Toolbox. available at http://www.mathworks.com/ products/statistics/, 2013. 
[69] K. Pelckmans et al., "Handling missing values in support vector machine classifiers," Neural Networks, vol. 18, no. 5, pp. 684-692, 2005.

[70] G. Chechik et al., "Max-margin classification of data with absent features," The Journal of Machine Learning Research, vol. 9, pp. 1-21, 2008.

[71] M. Saar-Tsechansky and F. Provost, "Handling missing values when applying classification models," Journal of machine learning research, 2007.

[72] E. R. Hruschka et al., "Evaluating a nearest-neighbor method to substitute continuous missing values," in Advances in Artificial Intelligence, pp. 723-734, Springer, Berlin Heidelberg, 2003.

[73] C.-C. Chang and C.-J. Lin, "LIBSVM: A library for support vector machines," ACM Transactions on Intelligent Systems and Technology, vol. 2, pp. 27:1-27:27, 2011. Software available at http://www.csie.ntu.edu.tw/ cjlin/libsvm.

[74] H. Peng, F. Long, and C. Ding, "Feature selection based on mutual information criteria of max-dependency, max-relevance, and min-redundancy," IEEE Transactions Pattern Analysis and Machine Intelligence, vol. 27, no. 8, pp. 1226-1238, 2005.

[75] A. Webb, Statistical Pattern Recognition. Wiley, 2003.

[76] E. Oja, A. Hyvarinen, and J. Karhunen, Independent Component Analysis. John Wiley \& Sons, 2001.

[77] T. Cover and J. Thomas, Elements of Information Theory. Wiley-interscience, 2006.

[78] I. Watson and F. Marir, "Case-based reasoning: A review," Knowledge Engineering Review, vol. 9, no. 4, pp. 327-354, 1994.

[79] M. P. Feret and J. I. Glasgow, "Combining case-based and model-based reasoning for the diagnosis of complex devices," Applied Intelligence, vol. 7, no. 1, pp. 57-78, 1997.

[80] L.-C. Wang, "Data mining in design and test processes: basic principles and promises," in Proceedings IEEE International Symposium on Physical Design, pp. 41-42, 2013.

[81] W. Klösgen and J. M. Zytkow, Handbook of data mining and knowledge discovery. Oxford University Press, Inc., 2002. 
[82] M. Steyvers and T. Griffiths, "Probabilistic topic models," Handbook of latent semantic analysis, vol. 427, no. 7, pp. 424-440, 2007.

[83] S. Pan and Q. Yang, "A survey on transfer learning," IEEE Trans. Knowledge and Data Engineering, vol. 22, no. 10, pp. 1345-1359, 2010.

[84] M. Hall, E. Frank, G. Holmes, B. Pfahringer, P. Reutemann, and I. H. Witten, "The weka data mining software: an update," ACM SIGKDD Explorations Newsletter, vol. 11, no. 1, pp. 10-18, 2009. 


\section{Biography}

\section{PERSONAL DATA}

- Fangming Ye

- Place of Birth: Shanghai, China

- Date of Birth: August 19, 1987

\section{EDUCATION}

- Ph.D., Duke University, USA, 2014

- M.S., Duke University, USA, 2011

- B.S., Fudan University, China, 2009

\section{PUBLICATIONS}

1. F. Ye, Z. Zhang, K. Chakrabarty, and X. Gu, "Board-level functional fault diagnosis using artificial neural networks, supportvector machines, and weighted-majority voting", IEEE Transactions on Computer-Aided Design of Integrated Circuits and Systems (TCAD), vol. 32, no. 5, pp. 723-736, May 2013.

2. F. Ye, Z. Zhang, K. Chakrabarty, and X. Gu, "Board-level functional fault diagnosis using multi-kernel support vector machines and incremental learning", IEEE Transactions on Computer-Aided Design of Integrated Circuits and Systems (TCAD), vol. 33, no. 2 pp. 279-290, February 2014. 
3. F. Ye, Z. Zhang, K. Chakrabarty, and X. Gu, "Information-theoretic framework for evaluating and guiding board-level functional fault diagnosis", IEEE Design 65 Test, vol. 31, no. 3, pp. 65-75, June 2014.

4. (*) K. Chakrabarty, M. Agrawal, S. Deutsch, B. Noia, R. Wang and F. Ye, "Test and design-for-testability solutions for 3D integrated circuits" (Invited Paper), Journal of Information Processing (Information Processing Society of Japan), vol. 7, pp. 55-73, August 2014.

5. (*) K. Chakrabarty, S. Deutsch, H. Thapliyal and F. Ye, "TSV defects and TSV-induced circuit failures: The third dimension in test and design-for-test" (invited paper), Proc. IEEE Reliability Physics Symposium, pp. 5F.1.1-5F.1.12, 2012.

6. (*) F. Ye and K. Chakrabarty, "TSV open defects in 3D integrated circuits: Characterization, test, and optimal spare allocation", Proc. IEEE/ACM Design Automation Conference, pp. 1024-1030, 2012

7. F. Ye, K. Chakrabarty, Z. Zhang and X. Gu, "Adaptive boardlevel functional fault diagnosis using decision trees", Proc. IEEE Asian Test Symposium, pp. 202-207, 2012.

8. F. Ye, K. Chakrabarty, Z. Zhang and X. Gu, "Board-level functional fault diagnosis using learning based on incremental supportvector machines", Proc. IEEE Asian Test Symposium, pp. 208$213,2012$.

9. (*) L. Jiang, Q. Xu, F. Ye, K. Chakrabarty and B. Eklow, "On effective and efficient in-field TSV repair for stacked 3D ICs", 
Proc. IEEE/ACM Design Automation Conference, pp. 1-6, 2013.

10. F. Ye, Z. Zhang, K. Chakrabarty and X. Gu, "Informationtheoretic syndrome and root-cause analysis for guiding board-level fault diagnosis", Proc. IEEE European Test Symposium, pp. 1-6, 2013 (Received Best Paper Award).

11. (*) F. Firouzi, F. Ye, K. Chakrabarty and M. B. Tahoori, "Representative critical-path selection for aging-induced delay monitoring", Proc. IEEE International Test Conference, pp. 1-10, 2013.

12. F. Ye, S. Jin, K. Chakrabarty, Z. Zhang and X. Gu, "Handling missing syndromes in board-level functional-fault diagnosis", Proc. IEEE Asian Test Symposium, pp. 73-78, 2013.

13. (*) F. Ye, F. Firouzi, Y. Yang, K. Chakrabarty and M. B. Tahoori, "On-chip voltage-droop prediction using support-vector machines", Proc. IEEE VLSI Test Symposium, pp. 282-287, 2014.

14. (*) F. Firouzi, F. Ye, K. Chakrabarty and M. B. Tahoori, "Chip health monitoring using machine learning" (invited paper), Proc. International Symposium on VLSI, 2014.

15. F. Ye, Z. Zhang, K. Chakrabarty and X. Gu, "Knowledge discovery and knowledge transfer in board-level functional fault diagnosis", accepted for publication in Proc. IEEE International Test Conference, 2014.

(*) Not related to PhD thesis work. 